\title{
Multi-Antenna Mobile Radio Channels: Modelling and System Performance Predictions
}

\author{
By: \\ Ghassan Dahman \\ BSc, MSc
}

A thesis submitted to the Faculty of Graduate

Studies and Research in partial fulfillment of the requirements for the degree of

\section{Doctor of Philosophy in Electrical and Computer Engineering}

\author{
Ottawa-Carleton Institute of Electrical and \\ Computer Engineering \\ Department of Systems and Computer Engineering \\ Carleton University \\ Ottawa, Ontario, Canada
}

May 2010

(C) Copyright 2010, Ghassan Dahman 


\section{Library and Archives Canada}

Published Heritage

Branch

395 Wellington Street Ottawa ON K1A ON4 Canada
Bibliotheque et

Archives Canada

Direction du

Patrimoine de l'édition

395 , rue Wellington Ottawa ON K1A ON4

Canada
Your file Votre refférence
ISBN: 978-0-494-67872-5
Our file Notre reférence
ISBN: $978-0-494-67872-5$
NOTICE:

The author has granted a nonexclusive license allowing Library and Archives Canada to reproduce, publish, archive, preserve, conserve, communicate to the public by telecommunication or on the Internet, loan, distribute and sell theses worldwide, for commercial or noncommercial purposes, in microform, paper, electronic and/or any other formats.

The author retains copyright ownership and moral rights in this thesis. Neither the thesis nor substantial extracts from it may be printed or otherwise reproduced without the author's permission.
AVIS:

L'auteur a accordé une licence non exclusive permettant à la Bibliothèque et Archives Canada de reproduire, publier, archiver, sauvegarder, conserver, transmettre au public par télécommunication ou par l'Internet, prêter, distribuer et vendre des thèses partout dans le monde, à des fins commerciales ou autres, sur support microforme, papier, électronique et/ou autres formats.

L'auteur conserve la propriété du droit d'auteur et des droits moraux qui protège cette thèse. $\mathrm{Ni}$ la thèse ni des extraits substantiels de celle-ci ne doivent être imprimés ou autrement reproduits sans son autorisation.
In compliance with the Canadian Privacy Act some supporting forms may have been removed from this thesis.

While these forms may be included in the document page count, their removal does not represent any loss of content from the thesis.
Conformément à la loi canadienne sur la protection de la vie privée, quelques formulaires secondaires ont été enlevés de cette thèse.

Bien que ces formulaires aient inclus dans la pagination, il n'y aura aucun contenu manquant.

\section{Canadä}


This thesis is dedicated to my parents and my wife for their love and endless support, and to my son, Omar. 


\section{Abstract}

Implementing multiple antennas at one or both ends of wireless communication systems has a significant impact on improving system performance. However, the gains in system capacity are sensitive to the spatial and temporal structure of the wireless channels upon which the system is built. The focus in this dissertation, is on using measurements to extract detailed information about the spatio-temporal behaviour of mobile wireless channels, modelling their dynamics, and utilizing this knowledge to improve transmission and receiving strategies.

The first part of the dissertation reports a measurement-based analysis that was conducted during the thesis project to characterize the dynamics of the spatial and temporal structure of wireless channels. Measurement data from a wideband single input multiple output channel sounding system with a 32-element uniform circular array that were recorded in a typical urban environment (downtown Ottawa) using a fixed transmitter and a moving receiver were analysed. Using a high resolution algorithm, the power, delay and angular parameters of the different multipath components were estimated. Following this identification of multipath parameters, major multipath clusters were identified, and their behaviour, such as the length of their active intervals, changes in their powers, as well as delay and angular characteristics within their active intervals were then modelled. The objective is to contribute to the development of a channel model having statistical characteristics that change in time to replace current models that generate outputs with the same statistics throughout total simulation intervals. It is believed that this will aid in the development of more efficient, adaptive, transmission techniques for use in future wideband systems.

In the second part of the dissertation, the information gained from studying the spatial structure of wireless channels is applied to improve the performance of multiple antenna systems. A new space division multiple access transmission technique is proposed, along with the specification of appropriate implementation algorithms. These algorithms utilize knowledge of the existence of multipath clusters, and angles of 
departure at a base station associated with transmission to specific users through selected clusters, for efficient beamforming in a hypothetical system, and it is demonstrated that the capacity is increased. Examples are considered in which the proposed transmission technique is applied in both broadcasting and cognitive networks.

In the final part of the dissertation, the information extracted from the SIMO sounding measurements is used to study the effect of several aspects of the receive antenna arrays on system performance. The extracted multipath parameters are used to reconstruct fields at the arrays under consideration, and subsequently study the effect of array configuration and element efficiency on mean effective link gain, eigenvalue dispersion and outage capacity under real-world channel conditions. Three configurations of a dual inverted-F receive antenna array in a generic mobile handset are studied. 


\section{Acknowledgements}

I would like, first and for most, to thank my supervisors Dr. Roshdy Hafez and Dr. Robert Bultitude for giving me this valuable opportunity to work with them. I could have never finished this work without their financial support, and endless technical help. This $\mathrm{Ph} . \mathrm{D}$. research opportunity was the greatest learning experience in my life. I was lucky to work with such great advisors with different specialties. Dr. Bultitude taught me how to be always eager to know more and more details about communication channels, and Dr. Hafez taught me to think always about how to use those details to improve the performance of communications systems. The frequent and fruitful discussions between the channel characterization point-of-view and the system performance point-of-view were certainly the source that enlightened the path of this research.

I am grateful to NSERC Canada for partial funding this research via Discovery Grant 299217-05. Also, I acknowledge the effort of the technical team at CRC in design and implementation of the channel sounder including Hong Zhu for work on the measurement system and participation in the measurements, Jeff Pugh for work in developing data collection system, and Tyler Smith for digital hardware development. 


\section{TABLE OF CONTENTS}

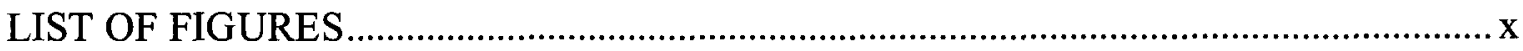

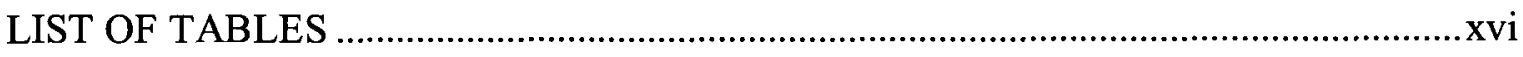

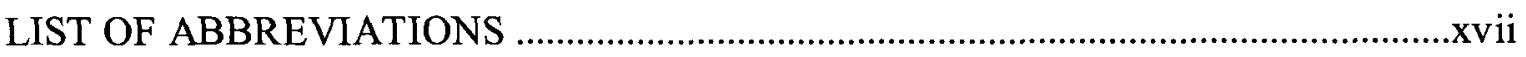

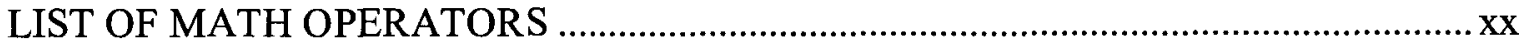

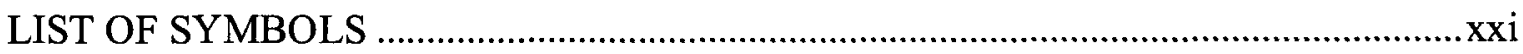

\section{CHAPTER 1: INTRODUCTION}

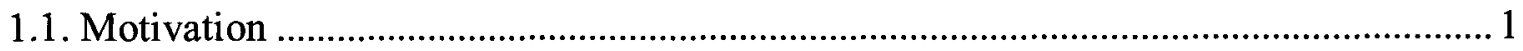

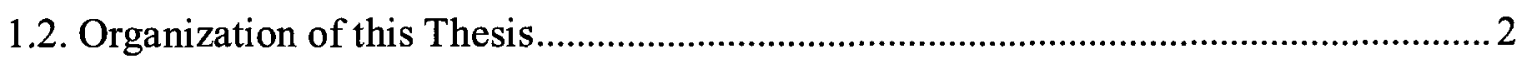

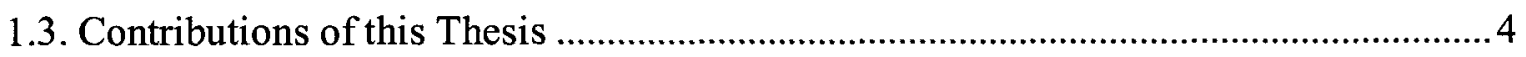

\section{CHAPTER 2: BACKGROUND}

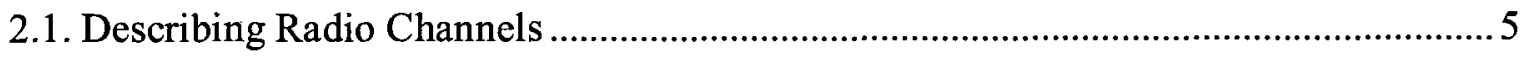

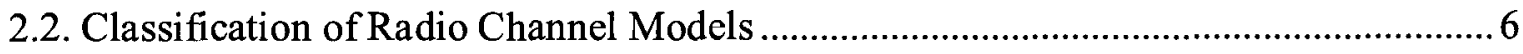

2.3. Standard Outdoor Multi-Antenna Channel Models ................................................. 8

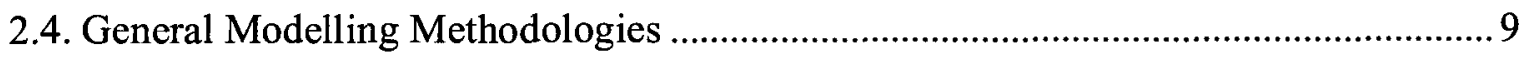

2.5. Simulating Changes in Wireless Channels............................................................. 11

2.6. Metrics to Detect Changes in Experimental Data ..................................................... 12

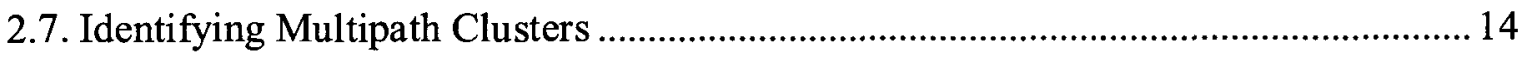

2.8. The Need for Measurement-Based Propagation Studies ........................................... 15

\section{CHAPTER 3: SINGLE-DIRECTIONAL RADIO CHANNEL MEASUREMENTS AND} DATA ANALYSIS

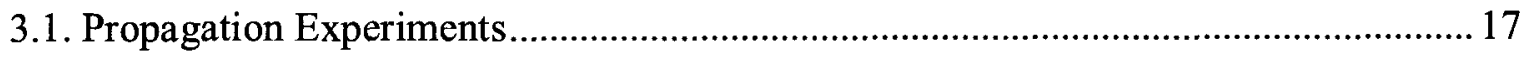

3.2. Sounder Specifications: CRC-Chanprobe ............................................................ 21

3.3. Estimation of the Parameters of MPCs Received at the CSMS .................................23

3.3.1. Estimation of Channel Impulse Response Functions .................................... 25

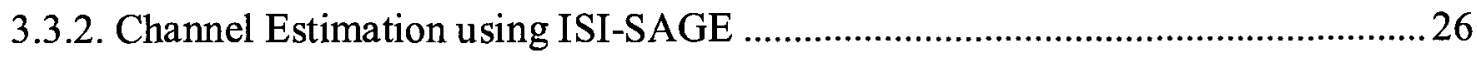

3.4. Evaluation of the Accuracy of Estimated Channel Parameters.................................. 30 


\section{CHAPTER 4: CLUSTER-BASED CHARACTERIZATION OF MICROCELLULAR} MOBILE RADIO CHANNELS

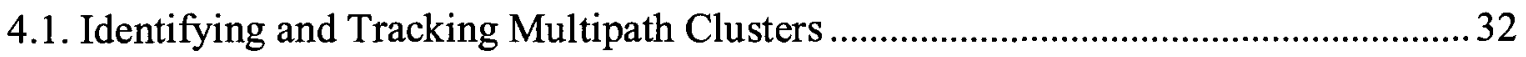

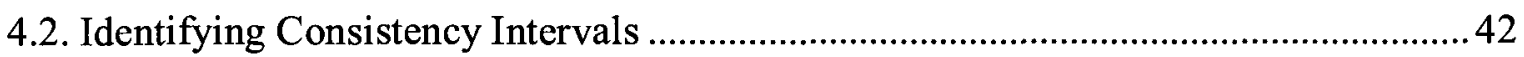

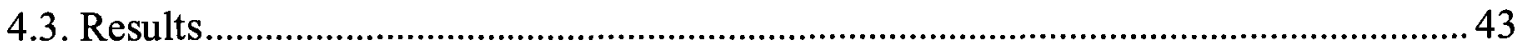

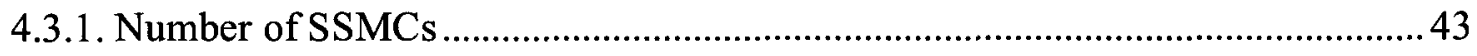

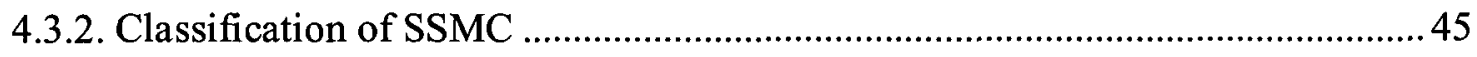

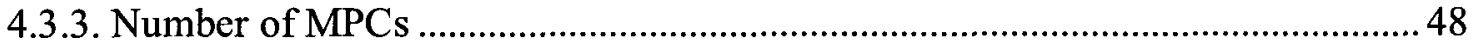

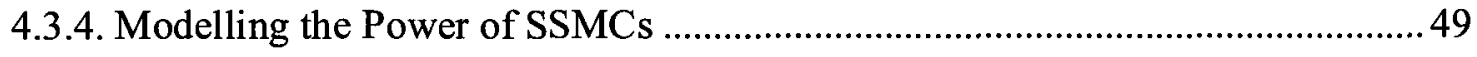

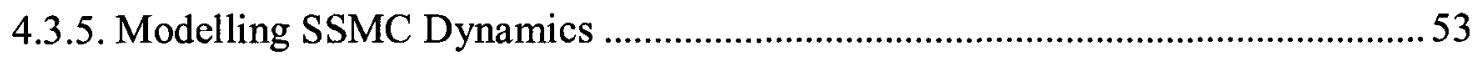

4.3.6. Comparing the Statistics of Consistency Intervals identified in Times series of measured data and in Simulation Results ..........................................58

4.4. Summary

CHAPTER 5: UTILIZING CLUSTERS FOR DYNAMIC SPATIAL OPPORTUNISTIC MULTIPLE ACCESS

5.1. The Clustering Nature of Wireless Channels 62

5.2. Angle-of-Departure-Aided Opportunistic Space-Division Multiple Access in MIMO Systems 63

5.2.1. Limited Feedback Techniques in Multiuser MIMO Broadcast Systems ................63

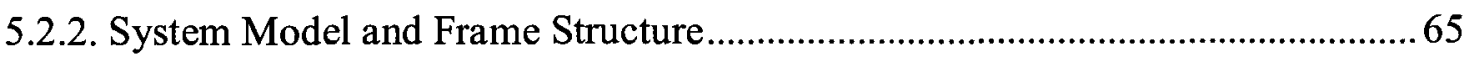

5.2.3. Angle-Of-Departure Estimation and Joint Tx-Rx Beamforming.......................66

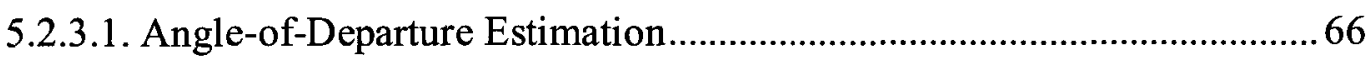

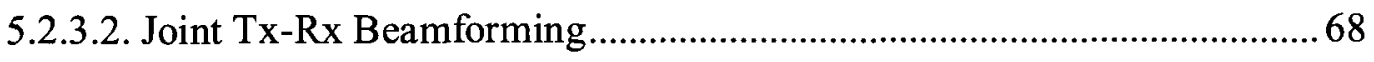

5.2.4. The Angle-Of-Departure Opportunistic Space-Division Multiple Access (AOD-OSDMA) Technique ........................................................... 70

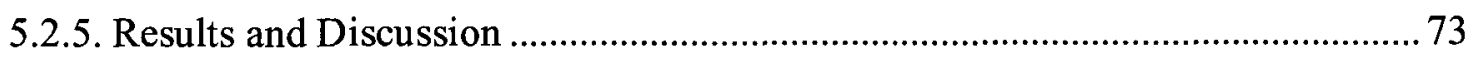

5.2.5.1. Comparison of System Performance when using the AOD-OSDMA, and OSDMA-S Techniques .74 
5.2.5.2. Comparison of the Number of Feedback Bits per User used with the Two Techniques 83

5.3. Modified Opportunistic Space-Division Multiple Access with Beam Selection 84

5.3.1. Motivation .84

5.3.2. System Model and Frame Structure. .84

5.3.3. The Modified Opportunistic Space-Division Multiple Access with Beam Selection (Mod-OSDMA-S) Technique .85

5.3.3.1 The Beam Design Criterion used in Mod-OSDMA-S 85

5.3.3.2. Selection of Users .86

5.3.3.3. Throughput Calculation .86

5.3.4. Results and Discussion .87

5.4. A Cluster-Based Spatial Opportunistic Spectrum Sharing technique for Cognitive Radio Applications. .91

5.4.1. Background: Cognitive Radio Technologies ..............................................91

5.4.2. System Model and Interference Estimation ......................................................92

5.4.3. The Frame Structure and Collaboration between Networks ..............................97

5.4.4. The Cluster-Based Spatial Opportunistic Spectrum Sharing Technique for Cognitive Radio Systems .97

5.4.4.1. Estimation of the AOD associated with the strongest clusters by the SUs .98

5.4.4.2. Transmission of Test Beams by the SBS. 101

5.4.4.3. Transmission of the Data Stream 102

5.4.5 Simulation Results 102

5.5. Summary 105

CHAPTER 6: EVALUATION OF THE COMMUNICATIONS PERFORMANCE ACHIEVABLE ON BASE STATION TO HANDHELD-TERMINAL RADIO LINKS WITH SEVERAL DIFFERENT PRACTICAL RECEIVE ANTENNA ARRAYS

6.1 Introduction 107 
6.2. The Synthesis of MIMO Radio Link Matrices using the ISI-SAGE

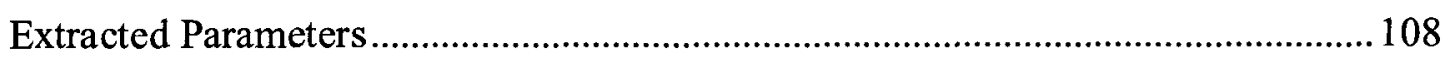

6.3. Estimation of MIMO Link Performance in the Hypothesised System.......................112

6.4. Antenna Configurations and HT Operating Positions that were Considered..............114

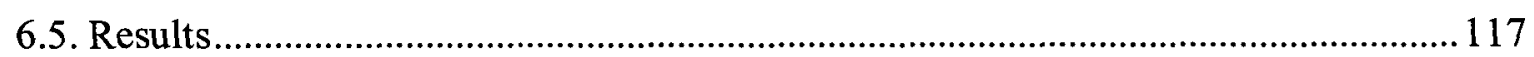

6.5.1. The Influence of Antenna Configuration, Antenna Efficiencies, and HT Position on $\mathrm{G}_{\text {ant }}$

6.5.2. The Influence of Antenna Configuration, Antenna Efficiency, and HT Position on Eigenvalue Dispersion

6.5.3. The Influence of Antenna Configuration, Antenna Efficiency, and HT Operating Position on BS-HT MIMO Link Outage Capacity

6.5.4. The Influence of Handheld Terminal Orientation on MIMO Link Performance Parameters

6.5.4.1. The Influence of HT Orientation, Antenna Configuration, Antenna Element Efficiency and HT Operating Position on $\mathrm{G}_{\text {ant }}$

6.5.4.2 The Influence of HT Orientation, Antenna Configuration, Antenna Element Efficiency and HT Operating Position on Eigenvalue

Dispersion

6.5.4.3. The Influence of HT Orientation, Antenna Configuration, Antenna Element Efficiency and HT Operating Position on Outage Capacity ...138

6.6. Summary 140

CHAPTER 7: SUMMARY, CONCLUSION AND TOPICS FOR FURTHER STUDY

7.1. Summary 142

7.2. Conclusion 143

7.3. Topics for Further Study 146

REFERENCES 148 


\section{LIST OF FIGURES}

3.1. Photograph showing the Tx configuration with the Tx monopole elevated to 6 metres above street level ............................ 18

3.2. Photograph of the 32 element UCA used for receiving during the reported experiments ..............................................

3.3. A view of the streets where propagation data were recorded illustrating the Tx location and some examples of the routs taken by the CSMS

3.4. The ECDF of Error ${ }_{\text {reconstruction }}$ calculated from 35,000 snapshots analysed using ISI-SAGE

4.1. Illustration of the steps used to track the different parameters of multipath clusters within a 1000-snapshot interval. (a) applying the proposed 2D clustering algorithm, and (b) tracking SSMCs ....

4.2. The sequential 2D clustering algorithm. (a) 3D illustration of an AMC where a vector $v$ represents the direction of interest that is used to extract a 1D waveform. (b) a plot of the extracted waveform in the direction of $v$ with the identified valley point in

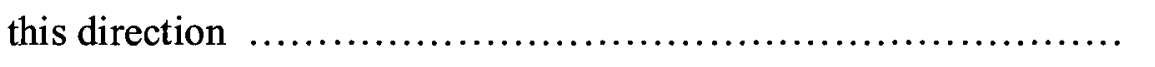

4.3. Examples of identified AMCs resulted from analysing propagation data using the proposed 2D clustering algorithm

4.4. CDF of the number of SSMCs. "Blue" color is measurements, and "Red" color is the best-fit Gamma model which has parameters $\mathrm{a}=4.27$, and $\mathrm{b}=2.10$

4.5. Lengths of active intervals that are less than 1000-snapshots long for different groups of SSMCs identified from measurement data.

(a) Group A, (b) Group B, and (c) Group C 
4.6. ECDFs for the number of MPCs per SSMC for Groups A, B, and C.

4.7. The ECDF and epdf of the powers of the SSMCs in the different groups with their GEV best-fit models. (a) Group A, (b) Group B, and (c) Group C

4.8. Comparison of the autocorrelation functions of the power values of SSMCs from measurements and simulations in different groups. (a) Group A, (b) Group B and (c) Group C

4.9. Comparison of the autocorrelation of time series derived from measurements and those derived from modelling for: (a) the rms DS of Group A. (b) the rms AS of Group A, (c) the rms DS of Group B, (d) the rms AS of Group B, (e) the rms DS of Group C, and (f) the rms AS of Group C

5.1. Comparison of system sum capacity when using AOD-OSDMA, and OSDMA-S with different inter-element spacings, as a function of the number of users: $\mathrm{SNR}=10 \mathrm{~dB}, \tau / L=0.02, \mathrm{~N}=4, \mathrm{M}=1$. (a) LOS and (b) NLOS

5.2. Comparison of system sum capacity when using AOD-OSDMA, and OSDMA-S with different inter-element spacings, as a function of the number of users: $\mathrm{SNR}=10 \mathrm{~dB}, \tau / L=0.02, \mathrm{~N}=8, \mathrm{M}=1$. (a) LOS and (b) NLOS

5.3. Effect of increasing the number elements at the user's terminals with $\mathrm{SNR}=10 \mathrm{~dB}, \tau / L=0.02$, LOS , and N=4 (a) AOD-OSDMA and (b) OSDMA-S

5.4. Effect of increasing the number elements at the user's terminals with $\mathrm{SNR}=10 \mathrm{~dB}, \tau / L=0.02$, LOS, and N=8 (a) AOD-OSDMA and (b) OSDMA-S 
5.5. Comparison of system capacity when using AOD-OSDMA, and OSDMA-S $(d=\lambda / 2)$ as a function of the number of users: $S N R=10$ $\mathrm{dB}, \tau / L=0.02, \mathrm{~N}=4, \mathrm{M}=2$. (a) LOS and (b) NLOS

5.6. Effect of increasing the number of bits per user (used for AOD feedback) on system capacity when using AOD-OSDMA with $\mathrm{SNR}=10 \mathrm{~dB}, \tau / L=0.02, \mathrm{~N}=4$, and $\mathrm{M}=2$. (a) LOS and (b) NLOS.. 80

5.7. Comparison of system capacity when using AOD-OSDMA, and OSDMA-S $(d=\lambda / 2)$ as a function of the number of users: $S N R=10$ $\mathrm{dB}, \tau / T=0.02, \mathrm{~N}=8, \mathrm{M}=2$. (a) LOS and (b) NLOS

5.8. Effect of increasing the number of bits per user (used for AOD feedback) on system capacity when using AOD-OSDMA with $\mathrm{SNR}=10 \mathrm{~dB}, \tau / T=0.02, \mathrm{~N}=8$, and $\mathrm{M}=2$. (a) LOS and (b) NLOS. 81

5.9. Comparison of system capacity when using AOD-OSDMA, and OSDMA-S $(d=\lambda / 2)$ as a function of the number of users: $S N R=10$ $\mathrm{dB}, \tau / T=0.02, \mathrm{~N}=8, \mathrm{M}=4$. (a) LOS and (b) NLOS

5.10. Effect of increasing the number of bits per user (used for AOD feedback) on system capacity when using AOD-OSDMA with $\mathrm{SNR}=10 \mathrm{~dB}, \tau / T=0.02, \mathrm{~N}=8$, and $\mathrm{M}=4$. (a) LOS and (b) NLOS.

5.11. Performance comparison of Mod-OSDMA-S and OSDMA-S as a function of the number of users using the WINNER model: $\mathrm{SNR}=10 \mathrm{~dB}, N=8$, and $\tau / L=0.02$.

5.12. Performance comparison of AOD-OSDMA, OSDMA-S, and modOSDMA-S (with $d=\lambda / 2$ for all techniques) as a function of the number of users with $\mathrm{SNR}=10 \mathrm{~dB}, \tau / L=0.02$, and $\mathrm{M}=1$. (a) $\mathrm{LOS}$, $\mathrm{N}=4$, (b) NLOS, $\mathrm{N}=4$, (c) LOS, $\mathrm{N}=8$, and (d) NLOS, $\mathrm{N}=8$.

5.13. System model. 
5.14. Effect of increasing the number of test beams on the sum capacity as a function of the total number of SUs. SBS 4 elements, SUs with 2 elements, $\gamma_{t h}=0.5 d B,\left(P_{S B S} / \sigma^{2}\right)=\left(P_{P U} / \sigma^{2}\right)=10 d B$, and $\left(\tau_{T}+\tau_{D}\right)=0.1 T$

5.15. Effect of changing the maximum allowed interference $\left(\gamma_{t h}\right)$ on the sum capacity as a function of the total number of SUs. SBS 4 elements, SUs with 4 elements, $\left(P_{S B S} / \sigma^{2}\right)=\left(P_{P U} / \sigma^{2}\right)=10 d B$, and $\left(\tau_{T}+\tau_{D}\right)=0.1 T$

6.1. The relative locations of the different antenna elements in $3 \mathrm{D}$ at the three different positions: (a) VP , (b) TP , and (c) DP...........

6.2. ECDF of $G_{a n t}$ at the VP for different configurations and element efficiencies combinations.

6.3. ECDF of $G_{a n t}$ at the DP for different configurations and element efficiencies combinations

6.4. ECDF of $G_{a n t}$ at the TP for different configurations and element efficiencies combinations

6.5. Power Azimuth Spectrum estimated using MPC parameters from all 15,000 propagation measurement snapshots

6.6. Azimuth antenna patterns for the different $\mathrm{Rx}$ antennas for different configuration/efficiency combinations. The solid line is for element\#1, and dashed line is for element $\# 2$.

6.7. $\mathrm{CDF}$ for $G_{m u x}$ at the VP for different configurations and element efficiencies combinations.

6.8. $\mathrm{CDF}$ for $G_{m u x}$ at the DP for different configurations and element efficiencies combinations 
6.9. $\mathrm{CDF}$ for $\mathrm{G}_{\mathrm{mux}}$ at the $\mathrm{TP}$ for different configurations and element efficiencies combinations.................................... 127

6.10. Outage capacity $C_{o}$ presented as a function of system SNR, for the case when the HT is in the Vertical operating position. Probability level $p_{O}=10 \%$

6.11. Outage capacity $C_{o}$ presented as a function of system SNR for the case when the HT is in the Data operating position. Probability level $p_{o}=10 \%$

6.12. ECDFs for the average $G_{\text {ant }}$ over 36 different azimuth orientations spaced by 10 degrees, when the HT is in the Vertical operating position

6.13. ECDFs for the greatest values of $G_{a n t}$ among the 36 values for different orientations of the HT estimated at every SL, when the HT is in the Vertical operating position.

6.14. ECDFs for the smallest values of $G_{a n t}$ among the 36 values for different orientations of the HT estimated at every SL, when the HF is in the Vertical operating position..........................

6.15. ECDFs for the average of $G_{m u x}$ for 36 orientations of the HT in azimuth, for various antenna configurations and efficiencies, when the HT is in the Vertical operating position...

6.16. ECDFs for the greatest values of $G_{m u x}$ for 36 orientations of the HT in azimuth, for various antenna configurations and efficiencies, when the HT is in the Vertical operating position...... 
6.17. ECDFs for the lowest values of $G_{m u x}$ for 36 orientations of the HT in azimuth, for various antenna configurations and efficiencies, when the HT is in the Vertical operating position................ 136

6.18. Outage capacity at an outage probability of $10 \%$, with the HT in the Vertical operating position............................... 138 


\section{LIST OF TABLES}

3.1. CRC-Chanprobe Operating Characteristics.................... 22

4.1. Parameters used to Model the Length of the Active intervals for the SSMC belonging to Different Groups........................ 46

4.2. The Percentage of SSMCs from Groups A, B, and C in Different AMC groups Identified in Measured Data........................ 47

4.3. The Values of Parameters of the Exponential Distribution used to Model the Length of Dormant Intervals for Different AMC groups................................................... 48

4.4. The Values of the Shape $(\xi)$, the Scale $(\sigma)$, and the Location $(\mu)$ Parameters used to Model the Powers of the SSMCs in Groups A, B, And C.

4.5. Comparison of the Means of the CIs Identified in Simulated Time Series Data and in the Measured Data........................... 60

5.1. Capacity Improvement of AOD-OSDMA Compared to OSDMA-S (with $d=\lambda / 2$ ) Capacities based on Simulations using the WINNER Model...

6.1. Example of Results (outage capacities) from the Analysis of One C-P-E-SNR Combination

6.2. Outage Capacities of the BX Configuration at Average System

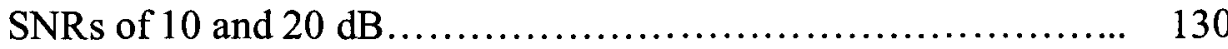

6.3. Medians for the Greatest Values of $\mathrm{G}_{\text {ant }}$ in Specified Groups of Results.

6.4. Medians for the Greatest Values of $\mathrm{G}_{\operatorname{mux}}$ in Specified Groups of Results. .

6.5. Outage Capacities for an Average System SNR of $10 \mathrm{~dB}$ for Specified Groups of Results..... 


\section{LIST OF ABBREVIATIONS}

$\begin{array}{ll}\text { AMC } & \text { Area Multipath Cluster } \\ \text { AOA } & \text { Angle of Arrival } \\ \text { AOD } & \text { Angle of Departure } \\ \text { AOD-OSDMA } & \text { Angle-of-Departure-Aided Opportunistic Space Division Multiple } \\ & \text { Access } \\ \text { AR } & \text { Autoregressive } \\ \text { AS } & \text { Angular Spread } \\ \text { BC } & \text { Bottom Co-polarized } \\ \text { BS } & \text { Base Station } \\ \text { BX } & \text { Bottom Cross-polarized } \\ \text { CI } & \text { Consistency Interval } \\ \text { CIR } & \text { Channel Impulse Response } \\ \text { COST } & \text { a European COoperative Scientific and Technical research forum } \\ \text { C-P-E-SNR } & \text { Configuration/Position/Efficiency/SNR } \\ \text { CRC } & \text { Communications Research Center } \\ \text { CSI } & \text { Channel State Information } \\ \text { CSMS } & \text { Channel Sounder Mobile Station } \\ \text { DP } & \text { Data Position } \\ \text { DS } & \text { Delay Spread } \\ \text { ECDF } & \text { Empirical Cumulative Distribution Function } \\ \text { GEV } & \text { Generalized Extreme Value } \\ \text { HT } & \text { Handheld Terminal } \\ \text { IO } & \text { Interacting Object } \\ \text { IRE } & \text { Impulse Response Estimate } \\ \text { ISI-SAGE } & \text { Initialization Search Improved Space-Alternating Generalized } \\ \text { KS } & \text { Expectation-maximization } \\ \text { LOS } & \text { Kolmogorov-Smirnov } \\ \text { MEG } & \text { Line Of Sight } \\ & \text { Mean Effective Gain } \\ & \end{array}$




\begin{tabular}{|c|c|}
\hline MELG & Mean Effective Link Gain \\
\hline MIMO & Multiple-Input Multiple-Output \\
\hline MISO & Multiple-Input Single-Output \\
\hline \multirow[t]{2}{*}{ Mod-OSDMA-S } & Modified Opportunistic Space division Multiple Access with beam \\
\hline & Selection \\
\hline MPC & Multipath Component \\
\hline MS & Mobile Station \\
\hline NLOS & Non Line Of Sight \\
\hline $\mathrm{OBF}$ & Opportunistic Beamforming \\
\hline OBF-S & Opportunistic Beamforming with beam Selection \\
\hline OGB & Opportunistic Grassmannian Beamforming \\
\hline OSDMA & Opportunistic Space-Division Multiple Access \\
\hline OSDMA-S & Opportunistic Space-Division Multiple Access with beam Selection \\
\hline OX & Opposite Cross-polarized \\
\hline PBS & Primary Base Station \\
\hline PU & Primary User \\
\hline PU-Rx & Primary User Receiver \\
\hline QoS & Quality of Service \\
\hline $\mathrm{Rx}$ & Receiver \\
\hline SBS & Secondary Base Station \\
\hline $\mathrm{SC}$ & Successive Cancellation \\
\hline $\mathrm{SCM}$ & Spatial Channel Model \\
\hline SCME & Spatial Channel Model Extended \\
\hline SIMO & Single-Input Multiple-Output \\
\hline SINR & Signal-to-Interference-plus-Noise Ratio \\
\hline SISO & Single-Input Single-Output \\
\hline SL & Street Location \\
\hline SNR & Signal-to-Noise Ratio \\
\hline SSMC & Single Snapshot Multipath Clusters \\
\hline SU & Secondary User \\
\hline
\end{tabular}


xix

$\begin{array}{ll}\text { SU-Rx } & \text { Secondary User Receiver } \\ \text { SU-Tx } & \text { Secondary User Transmitter } \\ \text { TP } & \text { Talk Position } \\ \text { Tx } & \text { Transmitter } \\ \text { UCA } & \text { Uniform Circular Array } \\ \text { VP } & \text { Vertical Position } \\ \text { WINNER } & \text { Wireless World Initiative New Radio } \\ \text { WSS } & \text { Wide Sense Stationary }\end{array}$




\section{LIST OF MATH OPERATORS}

$\begin{array}{ll}\|\ldots\|_{F} & \text { Frobenius norm } \\ \perp & \text { projection operation } \\ \ldots . & \text { absolute value } \\ (. .)^{H} & \text { Hermitian transpose } \\ (. .)^{*} & \text { complex conjugate } \\ * & \text { complex convolution } \\ \operatorname{tr}\{\ldots\} & \text { trace operator } \\ \otimes & \text { Kronecker product operator }\end{array}$




\section{LIST OF SYMBOLS}

\section{List of Symbols in Chapter 2}

$\begin{array}{ll}g_{R x}\left(\Omega_{R x}\right) & \text { receive antenna element patterns } \\ g_{T x}\left(\Omega_{T x}\right) & \text { transmit antenna element patterns } \\ h_{m, n}\left(t, \tau, \Omega_{T x}, \Omega_{R x}\right) & \begin{array}{l}\text { directional time-varying impulse response between the } n t h \text { transmit } \\ \text { element and the } m t h \text { receive element at time } t\end{array} \\ h_{m, n}(t, \tau) & \begin{array}{l}\text { non-directional time-varying impulse response between the } n t h \\ \text { transmit element and the } m t h \text { receive element at time } t\end{array} \\ \Omega_{R x} & \text { Solid angle of arrival } \\ \Omega_{T x} & \text { solid angle of departure } \\ S_{r T_{1}} & \text { powers in the } r t h \text { sub-interval of the spectrum corresponding to the } \\ & T_{1} \text { time instant }\end{array}$




\section{List of Symbols in Chapter 3}

\begin{tabular}{|c|c|}
\hline$\alpha_{\ell}$ & complex amplitude of the $\ell t h \mathrm{MPC}$ \\
\hline$h_{m}(\tau)$ & $\begin{array}{l}\text { impulse response of the radio link between the channel sounder } \\
\text { transmit and the } m t h \text { receive antennas }\end{array}$ \\
\hline$P_{f}$ & false alarm probability per sample \\
\hline$P_{F}$ & $\begin{array}{l}\text { false alarm probability per sample calculated using the } 32 \mathrm{Rx} \\
\text { elements }\end{array}$ \\
\hline$\varphi_{r x, \ell}$ & azimuth angle of arrival of the $\ell$ th MPC \\
\hline$\Psi_{\ell}$ & a vector with the parameters that characterize the $\ell$ th MPC \\
\hline$\hat{\Psi}_{\dot{\ell}}^{(0)}$ & vector of the initial estimates of the parameters of the $\dot{\ell} t h \mathrm{MPC}$ \\
\hline$s\left(t ; \hat{\Psi}_{\ell}^{(0)}\right)$ & contribution of the $\dot{\ell} t h$ MPC with parameters $\hat{\Psi}_{\dot{\ell}}^{(0)}$ to $y(t)$ \\
\hline$\sigma_{N}^{2}$ & variance of white noise \\
\hline$\sigma_{m}$ & estimated median level of the recorded samples at large delays \\
\hline$S V_{r x}\left(\varphi_{r x, \ell}, \theta_{r x, \ell}\right)$ & response of the Rx array to a MPC arriving from angles $\left(\varphi_{r x, \ell}, \theta_{r x, \ell}\right)$ \\
\hline$\tau_{\ell}$ & delay of the $\ell$ th MPC \\
\hline$\theta_{r, \ell}$ & elevation angle of arrival of the $\ell$ th MPC \\
\hline$T_{S C}$ & time duration of the sounding signal \\
\hline$u(t)$ & the complex baseband representation of the signal at the input of Tx \\
\hline$w(t)$ & $M$ dimensional temporally and spatially white noise \\
\hline$y(t)$ & signal at the output of $\mathrm{Rx}$ \\
\hline$y_{m}^{\ell}$ & portion of the $\ell t h$ signal that is received by the $m t h$ element \\
\hline$\zeta$ & thresholding level \\
\hline
\end{tabular}




\section{List of Symbols in Chapter 4}

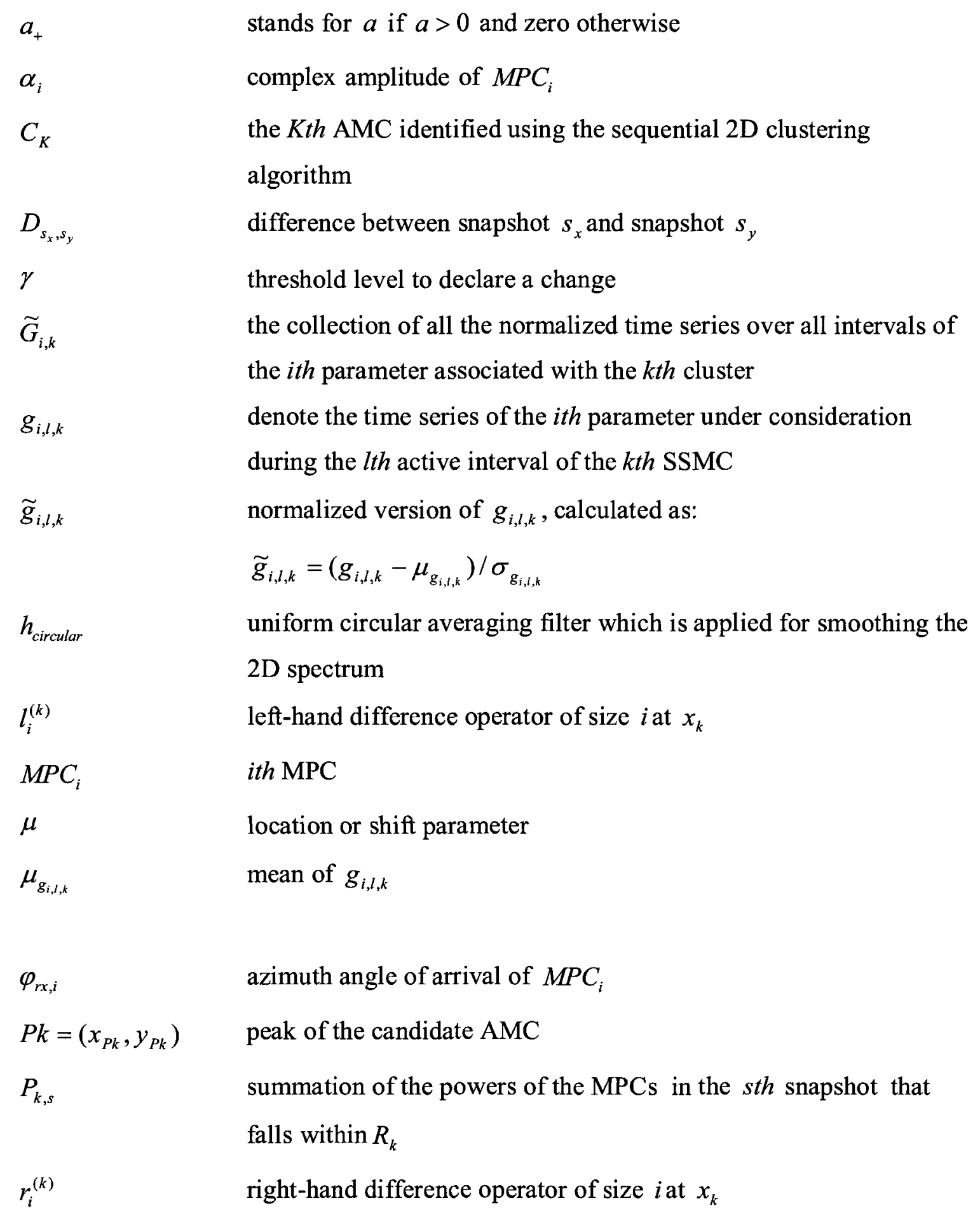




$\begin{array}{ll}R_{K} & \begin{array}{l}\text { the regions that contains the delay-angular coordinates of all the } \\ \text { points of the Kth AMC }\end{array} \\ & \text { normalized (unity power) 2D delay-angular spectrum results from } \\ & \text { averaging } 1000 \text { snapshots } \\ \sigma & \text { scale parameter } \\ \sigma_{g_{i, l, k}} & \text { standard deviation of } g_{i, l, k} \\ \tau & \text { propagation delay } \\ \tau_{i} & \text { Delay of } M P C_{i} \\ v & \text { vector on the delay-angular plane that starts at the point Pk in the } \varphi \\ & \text { direction } \\ W_{v} & \text { waveform which consists of all the points of } S \text { such that their } \\ x_{k} & \text { projection on the delay-angular plane intersect with the vector } v \\ & \text { The } k t h \text { point in the discrete waveform } x_{1}, \ldots . ., x_{N} \\ & \text { shape parameter }\end{array}$




\section{List of Symbols in Chapter 5}

\begin{tabular}{|c|c|}
\hline$a_{B S}$ & radius of the UCAs at the BS \\
\hline$\alpha_{l, S B S-S U k}$ & amplitude of the $l$-th MPC between the SBS and the $k t h$ SU \\
\hline$\alpha_{b, S B S-S U k}$ & amplitude of the $b$-th resolvable beam \\
\hline$\alpha_{b, u}$ & complex amplitude of the $b$-th resolvable beam \\
\hline$\alpha_{l, P B S-S U k}$ & amplitude of the $l$-th such MPC between the PBS and the $k t h \mathrm{SU}$ \\
\hline$\alpha_{l, S B S-P U}$ & amplitude of the $l$-th MPC between the SBS and the PU \\
\hline$\alpha_{l, u}$ & complex amplitude of the $l-t h$ MPC \\
\hline$a_{S B S}$ & radius of the UCA at the SBS \\
\hline$a_{S U k}$ & radius of the UCA at the $k t h \mathrm{SU}$ \\
\hline$a_{u}$ & radius of the UCA at the $u$-th user station \\
\hline$B_{S B S-S U k}$ & total number of resolvable beams linking the SBS and the $k t h$ SU \\
\hline$B_{u}$ & number of resolvable beams linking the BS and the $u$-th user \\
\hline$C_{S U k}$ & rate of the $k t h \mathrm{SU}$ \\
\hline$D$ & number of test beams sent by the SBS \\
\hline$\gamma_{t h r}$ & $\begin{array}{l}\text { maximum interference level that the PU can tolerate to meet the } \\
\text { required QoS }\end{array}$ \\
\hline$g_{P U}$ & path attenuation between the PBS and the PU \\
\hline$\hat{h}_{m}$ & $m$-th row of $\hat{H}_{S B S-S U k}^{M \times N}$ \\
\hline$H_{u}^{M \times N}$ & $\begin{array}{l}\text { multiple-input multiple-output channel between the BS and the } u t h \\
\text { user }\end{array}$ \\
\hline$\hat{H}_{u}^{M \times N}$ & $\begin{array}{l}\text { estimate of the multiple-input multiple-output channel between the } \\
\text { BS and the } u t h \text { user }\end{array}$ \\
\hline$\hat{H}_{S B S-S U k}^{M \times N}$ & $\begin{array}{l}\text { estimate of multiple-input multiple-output channel between the SBS } \\
\text { and the } k t h \mathrm{SU}\end{array}$ \\
\hline
\end{tabular}




\begin{tabular}{|c|c|}
\hline$H_{S B S-S U k}^{M \times N}$ & $\begin{array}{l}\text { multiple-input multiple-output channel between the SBS and the } \\
k t h \text { SU }\end{array}$ \\
\hline$\hat{h}_{m, u}$ & $m$-th row of $\hat{H}_{u}^{M \times N}$ \\
\hline$h_{m, n, u}$ & $\begin{array}{l}\text { matrix coefficient representing the channel between the } n \text {-th Tx } \\
\text { antenna at the BS and the } m \text {-th antenna at the } u \text {-th user station }\end{array}$ \\
\hline$h_{P B S-S U k}^{M \times 1}$ & $\begin{array}{l}\text { single-input multiple-output channel between the PBS and the } k t h \\
\text { SU }\end{array}$ \\
\hline$h_{S B S-P U}^{1 \times N}$ & multiple-input single-output channel between the SBS and the PU \\
\hline$h_{u}^{1 \times N}$ & $\begin{array}{l}\text { multiple-input single-output channel between the BS antennas and } \\
\text { the uth user }\end{array}$ \\
\hline$k$ & wavenumber at the operating frequency $(k=2 \pi / \lambda)$ \\
\hline$K_{\text {optimum }}$ & optimized number of the training minislots $(\mathrm{K})$ used by OSDMA-S \\
\hline$\lambda$ & wavelength at the operating frequency \\
\hline$L_{P B S-S U k}$ & $\begin{array}{l}\text { total number of MPCs associated with the channel between the PBS } \\
\text { and the kth SU }\end{array}$ \\
\hline$L_{S B S-P U}$ & $\begin{array}{l}\text { total number of MPCs associated with the channel between the SBS } \\
\text { and the PU }\end{array}$ \\
\hline$L_{u}$ & $\begin{array}{l}\text { total number of MPCs associated with the channel between the BS } \\
\text { and the } u \text {-th user }\end{array}$ \\
\hline$\Phi_{b, S B S-S U k}$ & phase of the $b$-th resolvable beam between the SBS and the $k$ th SU \\
\hline$\Phi_{b, u}$ & phase of the $b$-th resolvable beam between the BS and the $u t h$ user \\
\hline$\Phi_{l, P B S-S U k}$ & phase of the $l$-th MPC between the PBS and the $k t h$ SU \\
\hline$\Phi_{l, S B S-S U k}$ & phase of the l-th MPC between the SBS and the $k t h \mathrm{SU}$ \\
\hline$\Phi_{l, S B S-P U}$ & phase of the l-th MPC between the SBS and the PU \\
\hline$\Phi_{l, u}$ & phase of the $l$-th MPC between the BS and the $u t h$ user \\
\hline$\phi_{m, s U k}$ & angular position of the $m$-th element in the $k t h \mathrm{SU}$ array \\
\hline$\phi_{m, u}$ & angular position of the $m$-th element in the $u$-th user array \\
\hline
\end{tabular}


$\phi_{n, B S}$

$\phi_{n, S B S}$

$\varphi_{r x, b, S B S-S U k}$

$\varphi_{r x, b, u}$

$\varphi_{r x, l, P B S-S U k}$

$\varphi_{r x, l, S B S-S U k}$

$\varphi_{r x, l, u}$

$\varphi_{r x, s t, S B S-S U k}$

$\varphi_{t x, b, S B S-S U k}$

$\varphi_{t x, b, u}$

$\varphi_{t x, l, S B S-S U k}$

$\varphi_{t x, l, S B S-P U}$

$\varphi_{t x, l, u}$

$P_{P B S}$

$P_{S B S}$

$\rho$

$r_{u}$

$\sigma^{2}$

$\operatorname{SINR}_{u, n, k}$

$S I N R_{\max , S B S-S U k}$ angular position of the $n$-th element in the BS array

angular position of the $n$-th element in the SBS array

azimuth angle of arrival of the $b$-th resolvable beam

azimuth angle of arrival of the $b$-th resolvable beam

azimuth angle of arrival of the $l-t h$ MPC between the PBS and the $k$ th SU

azimuth angle of departure of the l-th MPC between the SBS and the kth $\mathrm{SU}$

azimuth angle of arrival of the $l$-th MPC

angle of arrival of the strongest cluster between the SBS and the

\section{$k t h \mathrm{SU}$}

azimuth angle of departure of the $b$-th resolvable beam

azimuth angle of departure of the $b$-th resolvable beam

azimuth angle of arrival of the $l$-th MPC between the SBS and the

\section{$k t h \mathrm{SU}$}

azimuth angle of departure of the $l$-th MPC between the SBS and the PU

azimuth angle of departure of the $l$-th MPC

transmit power of the PBS

transmit power of the SBS

input SNR at each user's terminal

correlation between the beamforming vector of the selected user and that of the $u$-th user

variance of $z_{P U}$ and $z_{S U k}$

SINR at the $u$-th user, resulting from receiving the $n$-th beam in the $k$-th training minislot,

SINR associated with the strongest cluster received by the $k t h S U$ 


\begin{tabular}{|c|c|}
\hline$S I N R_{P U}$ & SINR at the PU when the SBS is transmitting \\
\hline$S I N R_{S U k}$ & SINR at the $k$ th SU \\
\hline$s_{n}$ & pilot symbol sent by the $n t h \mathrm{Tx}$ antenna at the BS \\
\hline$S N R\left(\varphi_{t x, s t, u}, \varphi_{r x, s t, u}\right)$ & SNR associated with the strongest beam received by the $u$-th user \\
\hline$S N R_{P U}$ & SNR at the PU when there is no transmission from the SBS \\
\hline$s_{P U}$ & modulated signals for the PU \\
\hline$s_{S U k}$ & modulated signals for the $k t h \mathrm{SU}$ \\
\hline$S V_{\varphi_{x}, S B S}$ & the SBS element-space UCA steering vector in the $\varphi_{t x}$ direction \\
\hline$S V_{\varphi_{t x, s t}, S U k}^{1 \times N}, S B S$ & $\begin{array}{l}\text { SBS element-space UCA steering vector of the strongest cluster } \\
\text { associated with the kth } S U\end{array}$ \\
\hline$S V_{\varphi_{r}, S U k}^{1 \times M}$ & $\begin{array}{l}\text { element-space UCA steering vector at the } k t h S U \text { in the } \\
\varphi_{r x} \text { direction }\end{array}$ \\
\hline$S V_{\varphi_{t x}, B S}$ & BS element-space UCA steering vector in the $\varphi_{t x}$ direction \\
\hline$S V_{\varphi_{t x, s t u}, B S}$ & $\begin{array}{l}\text { BS element-space UCA steering vector of the strongest beam } \\
\text { associated with the } u \text {-th user }\end{array}$ \\
\hline$S V_{\varphi_{r}, u}$ & $\begin{array}{l}\text { element-space UCA steering vector at the } u \text {-th user station in the } \\
\varphi_{r x} \text { direction }\end{array}$ \\
\hline$\tau_{D}$ & $\begin{array}{l}\text { length of the delay minislot that is needed by the SBS to send test } \\
\text { beams and receive feedback from the PU }\end{array}$ \\
\hline$\tau_{T}$ & total length of the training minislots that are sent by SBS \\
\hline$U$ & total number of users \\
\hline$w_{S B S-S U k}^{1 \times N}$ & beamforming weight vectors at the SBS \\
\hline$w_{S U k}^{1 \times M}$ & beamforming weight vectors at the $k t h \mathrm{SU}$, \\
\hline$\Xi_{u}^{M \times 1}$ & complex zero-mean additive white Gaussian noise \\
\hline$y_{P U}$ & received signal at the primary user \\
\hline$y_{S U k}^{M \times 1}$ & received signal of the $k t h \mathrm{SU}$ \\
\hline
\end{tabular}


xxix

$z_{P U}$

$z_{s U k}$

zero-mean i.i.d. additive white Gaussian noise processes at the PU zero-mean i.i.d. additive white Gaussian noise processes at the $k t h$ $\mathrm{SU}$

List of Symbols in Chapter 5 


\section{List of Symbols in Chapter 6}

\begin{tabular}{|c|c|}
\hline$\alpha_{\ell}$ & complex amplitude of the $\ell$ th MPC \\
\hline$C_{O}$ & outage capacity \\
\hline$D_{r x}\left(\theta_{r x, \ell}, \varphi_{r x, \ell}\right)[m]$ & $\begin{array}{l}\text { the directivity of the } m t h \text { element of the Rx antenna in the } \\
\left(\theta_{r x, \ell}, \varphi_{r x, \ell}\right) \text { direction, corresponding to the } \ell t h \mathrm{MPC}\end{array}$ \\
\hline$E f f[m]$ & $\begin{array}{l}\text { the sum of the mismatch and radiation efficiencies, or total } \\
\text { efficiency of the } m t h \text { element in the Rx antenna array }\end{array}$ \\
\hline$E_{\theta}\left(\theta_{r x, \ell}, \varphi_{r x, \ell}\right)[m]$ & $\begin{array}{l}\text { the vertically polarised component of the E field of the } m t h \mathrm{Rx} \\
\text { antenna element in the direction }\left(\theta_{r x, \ell}, \varphi_{r x, \ell}\right) \text {, corresponding to the } \\
\text { eth MPC }\end{array}$ \\
\hline$\underset{\bar{z}}{F_{\theta}}\left(\theta_{r x, \ell}, \varphi_{r x, \ell}\right)[m]$ & $\begin{array}{l}\text { the vertically polarized component of the complex electromagnetic } \\
\text { field pattern of the } m t h \mathrm{Rx} \text { antenna element in the direction } \\
\left(\theta_{r x, \ell}, \varphi_{r x, \ell}\right) \text {, corresponding to the } \ell \text { th } \mathrm{MPC}\end{array}$ \\
\hline $\mathrm{G}_{\text {ant }}$ & effective link gain \\
\hline $\mathrm{G}_{\mathrm{mux}}$ & eigenvalue dispersion \\
\hline $\begin{array}{l}g_{t x}\left(\theta_{t x, \ell}, \varphi_{t x, \ell}\right)[n] \\
H^{(i)}\end{array}$ & $\begin{array}{l}\text { gain of the } n t h \mathrm{Tx} \text { antenna element in the }\left(\theta_{t x, \ell}, \varphi_{t x, \ell}\right) \text { direction. } \\
\text { the channel matrix of the } i t h \text { snapshot }\end{array}$ \\
\hline$H_{i s o}^{(i)}$ & $\begin{array}{l}\text { the } H^{(i)} \text { matrix evaluated using isotropic radiators for the elements } \\
\text { of the HT and BS antennas. }\end{array}$ \\
\hline$H_{i s o, s l i}^{(i)}$ & $H_{i s o}^{(i)}$ calculated over some sliding spatial interval \\
\hline$h_{m n}(\tau)$ & $\begin{array}{l}\text { time-invariant single-input single-output impulse response of the } \\
\text { radio link between the } n t h \mathrm{Tx} \text { antenna element and the } m t h \mathrm{Rx} \\
\text { antenna element }\end{array}$ \\
\hline$I_{H}^{(i)}$ & the mutual information associated with $H^{(i)}$ \\
\hline $\mathrm{L}$ & total number of received MPCs \\
\hline$\lambda_{k}^{(i)}$ & $k t h$ eigenvalue of $H^{(i)}$ \\
\hline$M$ & number of receive antennas \\
\hline & number of transmit antennas \\
\hline
\end{tabular}




\begin{tabular}{|c|c|}
\hline$N_{s}$ & total number of processed snapshots \\
\hline$N_{s n}$ & $\left(N_{s n}+1\right)$ is the width of the averaging window \\
\hline$P_{\text {norm }}$ & normalization factor used when calculating the mutual information \\
\hline & associated with the channel matrix $H^{(i)}$ \\
\hline$p_{O}$ & the probability level used to evaluate the outage probability at \\
\hline$\theta_{t x, \ell}$ & elevation angle-of-departure for the lth MPC \\
\hline$v_{r x}\left(\theta_{r x, \ell}, \varphi_{r x, \ell}\right)[m]$ & $m t h$ element of the $\mathrm{Rx}$ antenna steering vector corresponding to the \\
\hline & $\ell t h \mathrm{MPC}$ \\
\hline$v_{t x}\left(\theta_{t x, \ell}, \varphi_{t x, \ell}\right)[n]$ & nth element of the Tx antenna steering vector corresponding to the \\
\hline & eth MPC \\
\hline$y_{m n}$ & complex envelope of the narrow band received signal between the \\
\hline & $n t h \mathrm{Tx}$ antenna element and the $m t h \mathrm{Rx}$ antenna element \\
\hline
\end{tabular}




\section{CHAPTER 1:}

\section{INTRODUCTION}

\subsection{Motivation}

Wireless communications systems face increasing demands for faster and higher throughputs due to heavy usage and capacity-intensive data applications. The limited radio spectrum forms a major obstacle to achieving the needed high-data rates. The use of multiple antennas at one or both ends of the communications link has emerged as one of the most promising techniques for providing greater capacity and better performance.

Because wireless systems are so sensitive to the spatio-temporal characteristics of wireless channels, several factors can improve their performance and efficiency in the future. Among these factors are:

a) Improving the accuracy of channel modelling used in evaluating the performance of multiple antenna systems. The most useful channel model should realistically describe (1) the important physical parameters of radio propagation channels, such as the direction and delay of received Multipath Components (MPCs) and (2) the effect of the time-varying behaviour of the channel, which is influenced by the motion of mobile terminals and surrounding objects in the operating environment.

b) Improving signal processing techniques such that they consider characteristics of real-world wireless channels (e.g., the existence of multipath clusters) instead of assuming purely theoretical and sometimes unrealistic characteristics.

c) Improving the design of future antennas at both ends of the communications links in such a way that their interactions with wireless channels improve the overall performance of communications systems. Some of the parameters of multiple antennas that can be considered: radiation patterns, polarization, element efficiency, and position- and inter-antenna-element spacing within a communication device. 
Making contributions to the abovementioned methods by which the efficiency and performance of communications systems can be improved is the primary motivation for the work done during the thesis project reported on herein. In this thesis, special attention has been paid to the description and characterization of multipath clusters based on a measurement-based study. The information estimated from measured data is used to evaluate different base- and mobile-station antenna configurations, and to develop new signalling techniques that take advantage of the spatial distribution of multipath clusters in the environment.

\subsection{Organization of this Thesis}

Chapter 2 provides background material about modelling radio channels. Section 2.1. discusses the different ways used to describe wireless channels. Sections 2.2. and 2.3. describe the classification of wireless channel models and the standard outdoor channel models, respectively. Section 2.4. explains the basic modelling methodologies behind the current wireless channel models. Sections 2.5. and 2.6. list the current approaches that are used to model changes in wireless channels and the metrics that are used to detect changes in experimental data, respectively. Section 2.7. describes the methods that are used to identify multipath clusters. Section 2.8. justifies the need for more measurementbased studies to extract detailed information of wireless channels.

Chapter 3 describes the propagation experiments that were conducted in downtown Ottawa to record channel sounder data. These data were later processed to obtain estimates of the parameters of MPCs received at the Channel Sounder Mobile Station (CSMS). Section 3.1. describes the propagation experiments. Sections 3.2. and 3.3. report the specifications of the channel sounder and the procedure used to extract the parameters of the MPCs, respectively. Section 3.4. reports an evaluation of the accuracy achievable with methods used during the thesis project for estimating channel parameters. 
Chapter 4 focuses on the clustering nature of wireless channels as an effective tool that can be used in order to model the time evolution of wireless channels based on measured data. In Section 4.1., an algorithm for the identification of multipath clusters is introduced. In Section 4.2., the concept of using consistency intervals to describe intervals between changes in channel characteristics is reviewed. Section 4.3. reports results from analyzing propagation measurement data. This section also includes statistics on (a) the number of clusters, (b) the instantaneous values of the different parameters of clusters, (c) the number of rays per cluster, and (d) the modelling of the time-varying behaviour of clusters.

Chapter 5 introduces different techniques that utilize knowledge of the existence of multipath clusters to design systems that improve communication system throughput. Section 5.1. explains the clustering nature of wireless channels. Sections 5.2 and 5.3 describe the use of multipath clusters in multiple antenna broadcasting channels (i.e., in systems where multiple antennas at a BS are sending information to multiple Rxs). Section 5.4. considers utilizing multipath clusters in cognitive radio systems.

Chapter 6 presents research in which empirical data were used to evaluate the performance of MIMO radio links in which user-handheld terminals had a number of alternate receive antennas. Section 6.2 discusses the method used to synthesize MIMO channel matrices from the estimated parameters of MPCs received during propagation experiments. Section 6.3 describes the estimation of effective link gain, eigenvalue dispersion and radio link capacities, all of which have considerable influence on MIMO radio link performance. Section 6.4 describes handheld terminal-antenna-mounting positions and array configurations, as well as three different handheld-terminal operating positions that were considered. Section 6.5. presents the performance-evaluation results.

Chapter 7 concludes the work of this thesis. Sections 7.1. and 7.2. give a summary of the results and conclusions. Section 7.3. suggests topics for further study. 


\subsection{Contributions of this Thesis}

The main contributions of this thesis are:

1. Introducing algorithms to identify multipath clusters in wireless channels and to model the dynamics of their angular and delay parameters. This includes:

- Proposing an algorithm to identify multipath clusters and track their number, characterize the dynamics of the change in their powers, Angular Spreads (AS), and Delay Spreads (DS).

- Introducing a new method to identify the points where the delay-angular characteristics of the channel changes. Consequently, the lengths of consistency intervals were modeled.

- Introducing a new algorithm to model the power of multipath clusters during their active intervals.

2. The specification of techniques for improving the performance of multiple antenna systems by considering the characteristics of real-world channels. This includes:

- Introducing a novel transmission technique for use on multiple-input multiple-output (MIMO) broadcasting channels, in which the system takes advantage of multiuser diversity that results from the existence of multipath clusters.

- Introducing a novel technique that allows the coexistence of secondary users with a primary user simultaneously in the same frequency band, in cognitive radio systems having a spectrum sharing setup of the underlying type.

- Evaluating the influence of handheld terminal receive antenna element placement, radiation patterns, polarization, and efficiency on effective link gain in a multipath environment, MIMO link multiplexing gains, and MIMO link outage capacity. 


\section{CHAPTER 2:}

\section{BACKGROUND}

This chapter provides background material about modelling radio channels. Section 2.1. discusses the different ways used to describe radio channels. Sections 2.2. and 2.3. describe the classification of radio channel models and the standard outdoor multi-antenna channel models, respectively. Section 2.4. explains the basic modelling methodologies behind the current channel models. Sections 2.5. and 2.6. list the current approaches that are used to model changes in wireless channels and the metrics that are used to detect changes in experimental data, respectively. Section 2.7. describes the methods that are used to identify multipath clusters. Section 2.8 . justifies the need for more measurement-based studies to extract detailed information of wireless channels.

\subsection{Describing Radio Channels}

Wireless radio channels are known for their complex, random spatial and temporal variations. The difficulty in knowing exact details about a particular channel presents a real challenge in the achievement of the maximum possible capacity for multiple antenna systems. The randomness of radio channels comes from scattering, reflection, and diffraction phenomena, which result in the reception of a number of replicas of the transmitted signal over different propagation paths, i.e., MPCs. Moreover, the parameters of the MPCs vary with time because of the stochastic movement of the radio terminals, as well as interacting objects (IOs) in the environment of operation. Each MPC can be fully characterized by its complex amplitude, angle-of-arrival (AOA) at the receiver (Rx), angle-of-departure (AOD) at the transmitter (Tx), and delay. Consequently, a multipleinput multiple-output (MIMO) propagation channel can be characterized by its matriximpulse response, which describes the channel as a matrix of impulse responses between individual transmit-receive antenna element pairs. The latter is calculated as the vector sum of the contribution of the all MPCs with powers above the noise level [1]. Descriptions of MIMO propagation channels exclude the characteristics of the $\mathrm{Tx}$ and $\mathrm{Rx}$ 
antennas and focus instead on the properties of the propagation radio links among antennas. On the other hand, a MIMO channel matrix describes the effect of both the propagation channel and the antenna characteristics at the two ends of a communications link.

The relationship between these two conceptualizations for time-varying channels can be established as:

$$
h_{m, n}(t, \tau)=\iint_{\Omega_{T_{x}}} \int_{\Omega_{x x}} h_{m, n}\left(t, \tau, \Omega_{T x}, \Omega_{R x}\right) d \Omega_{R x} d \Omega_{T_{x}},
$$

where $h_{m, n}(t, \tau)$ denotes the non-directional time-varying impulse response between the $n t h$ transmit element and the $m t h$ receive element. $h_{m, n}\left(t, \tau, \Omega_{T x}, \Omega_{R x}\right)$ is the directional time-varying impulse response of the radio link between the nth transmit element and the mth receive element at time $t, \tau$ is the propagation delay, $\Omega_{T_{x}}$ and $\Omega_{R x}$ are the solid AOD and AOA respectively. The impulse response functions $h_{m, n}\left(t, \tau, \Omega_{T x}, \Omega_{R x}\right)$ can be estimated from radio propagation measurements.

\subsection{Classification of Radio Channel Models}

Radio-channel models are important tools in the assessment of the performance of communication systems. This assessment can be done by generating either MIMO channel matrices or multiple MPCs with spatio-temporal properties that reflect the actual spatio-temporal components of MIMO channels. Several methods for classifying radiochannel models have been proposed in the literature using different classification criteria [2 - 6]. In general, radio-channel models can be classified into nonphysical and physical models.

The non-physical-based modelling approaches are also called analytical models [2], matrix characterization models [6] or stochastic models [3], and their outcome is the statistical characterization of the channel matrix. 
On the other hand, the physical models (also called multipath-characterization models [6]) aim to describe the physical multipath propagation channel; they can further be divided into deterministic-physical models and stochastic-physical models.

The most simple nonphysical model is one that models all the elements of the channel matrix as independent, identically distributed (i.i.d.) zero-mean Gaussian random variables with equal variance. This model is spatially white, i.e., it corresponds to an ideal dense multipath environment. Other models have also been introduced in [7 - 9] based on the assumption that channel coefficients are components of a multivariate complex Gaussian distribution.

The complete description of the multivariate complex Gaussian model requires knowledge of the full correlation matrix, thus leading to significant complexity. To reduce the computational complexity involved in calculating the full matrix correlation, the Kronecker model was introduced in order to model and analyze the capacity of the channel $[4,10]$. This model uses a separability assumption to simplify the expression of the full correlation matrix $\left(R_{\text {Full }}\right)$ as follows.

$$
R_{\text {Full }}=R_{r} \otimes R_{t}
$$

where $\otimes$ is the Kronecker product operator, $R_{r}$ and $R_{t}$ are the transmit and receive correlation matrices, respectively.

The Kronecker model is valid if and only if two conditions are met: 1) the transmit correlation coefficients are independent from the considered receive antenna, and 2) the cross-channel correlations must be equal to the product of corresponding transmit and receive correlations [11]. This model results in underestimation of the channel capacity when large arrays are used [11 - 13]. To overcome the Kronecker deficiencies, Weichselberger introduced a model that results in great flexibility in the degree of diversity, and multiplexing gain, and overcomes the problem of underestimating the channel capacity $[14,15]$.

Physical modelling approaches aim to describe the physical real-world characteristics of radio channels such as the MPCs and their parameters (i.e., AOA, AOD, power and delay) independently from system aspects such as antenna properties or system 
bandwidth. These models reflect the effect of the channel on the radio signal, and can be classified into deterministic-physical models and stochastic-physical models.

The deterministic-physical models tend to reproduce the actual radio propagation process for a certain environment by either playback of actual recorded measurements or running simulations using ray-tracing, where the later techniques simulate the radio propagation process using Maxwell's equations or, simply, the optical approximation. The implementations of these models require significant computational power and a great many details about the environment.

The stochastic-physical models construct a realistic geometric environment of IOs and generate the corresponding MPCs accordingly. One approach is to obtain the statistics of the number and locations of IOs in a stochastic fashion according to a certain probability distribution that reflects physical reality. The actual parameters of the MPCs are reproduced using a simple ray-tracer, assuming certain conditions (e.g., one ring of IOs, two rings of IOs, single-interaction or multiple interactions) $[5,16,17]$.

Measurements are also used to determine stochastic-physical models for mobile radio channels. In this approach the channel is probed using a known signal, and then the raw measurement data at $\mathrm{Tx}$ and/or $\mathrm{Rx}$ are analyzed to extract the channel details. This approach requires sophisticated equipment to capture and analyze measured data to estimate the parameters of the MPCs such as AOAs, AODs, powers and delays which are then described in terms of their statistics $[18,19]$.

\subsection{Standard Outdoor Multi-Antenna Channel Models}

This section describes the main properties of some of the standard MIMO channel models for outdoor scenarios. Models discussed include the: COST 259, COST 273, SCM, SCME, WINNERI and WINNERII models.

The COST 259 "Flexible personalized wireless communications" and COST 273 "Towards mobile broadband multimedia networks" models were initiated by a European COoperative Scientific and Technical research forum (COST) the objective of which was to develop directional MIMO channel models suitable for simulations of smart antenna systems for a wide range of indoor and outdoor radio environments. The COST 259 
model is a parametric stochastic model that covers frequencies from $800 \mathrm{MHz}$ up to 2 $\mathrm{GHz}$ (for outdoors) and $800 \mathrm{MHz}$ up to $5 \mathrm{GHz}$ (for indoors). The COST 273 model covers frequencies from $10 \mathrm{GHz}$ to $60 \mathrm{GHz}[20,21]$. The COST 259 and COST 273 models show general similarities, but the latter parameterizes additional new operating environments, and uses updated measurement-based parameters and different distributions for AOAs and AODs [2]. The Spatial Channel Model (SCM) was developed by the $3 \mathrm{GPP} / 3 \mathrm{GPP} 2$ groups to specify parameters and methods associated with outdoor channel modelling at a centre frequency of $2 \mathrm{GHz}$ and bandwidth of $5 \mathrm{MHz}$. The SCM model uses both geometric and stochastic approaches for link- and system-level evaluation [22]. The SCME model was introduced as a direct extension of the SCM model to allow the model to cover centre frequencies in the range of 2 and $6 \mathrm{GHz}$ and bandwidths up to $100 \mathrm{MHz}$. This extension approach required backward-compatibility with the SCM model, which resulted in unrealistic assumptions such as that the number of clusters and MPCs should be the same as for the SCM model despite the increase in the bandwidth.

The WINNER I "Wireless World Initiative New Radio" model was developed to solve drawbacks in the SCME model, to compensate for the lack of measurement-based wideband system-level models, and to come up with a channel model for Beyond-3G (B3G) radio systems using a bandwidth of $100 \mathrm{MHz}$ for each radio link and cover frequencies between 2 and $6 \mathrm{GHz}$ [23]. The WINNER II model set is an extension of the WINNER I model set, including the definition of more parameter sets for different operating environments, more parameters (e.g., AOAs and AODs in elevation) and a crude form of time evolution of the model parameters [24].

\subsection{General Modelling Methodologies}

The basic modelling methodology behind the COST 259, COST 273, SCM, SCME and WINNER models is to characterize the radio channel in delay and angle using a directional (or double-directional) channel impulse response that is constructed as the contribution of all individual MPCs with power above the system-noise level $[25,26]$. 
All of the abovementioned models are classified as physical-stochastic channel models i.e., the parameters of each MPC (e.g. power, delay, AOA, AOD) are selected randomly from appropriate distributions, which are determined according to global or large-scale parameters. In the case of the COST 259, COST 273, and SCM models, the parameter distributions result from a combination of measurements and ray-tracing simulations. In contrast, the WINNER parameter distributions result only from measurements. In the case of smaller system resolution (COST 259 case), the channelimpulse response is described in terms of the statistics of the resolvable multipath groups. All models are structured using a multi-level/hieratical structure, in which each layer defines - deterministically or statistically — different types of parameters.

The COST 259 model uses a 3-level structure to define three types of radio environment (Macro-, Micro- and Pico- cellular) and 13 different generalized radio environments (e.g., typical urban, bad urban, open place, and factory/hall). Each generalized radio environment is associated with a whole set of propagation conditions. The last layer of the hierarchal structure consists of the propagation scenarios, which are defined as random realizations of multipath conditions with constant global parameters. The COST 259 model requires the specification of three types of parameters, including: (a) external parameters to describe the radio environment (for example, average BS and MS antenna height, average BS-to-MS distance, average height of surrounding buildings, frequency band and specification, statistics of line-of-sight (LOS) or none-line-of-sight (NLOS) conditions); (b) global parameters which were extracted from measurements or ray-tracing simulations to describe the propagation conditions of each radio environment such as the number of the IOs; and (c) local parameters: to describe the instantaneous channel conditions in a small local area where the local parameters represent realizations of the global parameters.

Using a similar approach, the SCM and WINNER models generate MPCs by: (a) specifying an environment which determines the large-scale parameters such as the path loss, mean AS and mean DS at the BS and MS, and number of clusters; (b) specifying the parameters to be used in simulations such as BS to MS distance and orientation; and (c) generating the parameters to be used in simulations through random variable generation 
equations specified for the corresponding scenario and large-scale parameters (e.g., power, delay, AOAs, AODs and phases for each MPC) [25, 27].

\subsection{Simulating Changes in Wireless Channels}

In [28] there was a suggestion to stop assuming that the channel has the same properties all the time and adopt a more realistic assumption in which the simulation is done in intervals, in each of which radio channel variations can be represented by different stochastic parameters. For example:

- The COST 259 model assumes that both the IOs and the Tx are stationary, and that all the channel time-variations are due solely to movement of the $\mathrm{Rx}$, which is not the case in many real-world environments. SCM and WINNER I system simulation is carried out as a sequence of "segments" or "drops." For each channel segment/drop it is assumed that: (a) the large-scale parameters such as AS, DS, mean AOA and shadowing are constant, (b) the virtual motion of the MS causes the channel to experience fast fading due to the superposition of rotating phasors, and (c) the location of the MS varies randomly before the beginning of each segment/drop.

- The SCME model addresses the channel time-evolution by virtually extending the time duration of SCM-drops. This was achieved by adding short-term time-variability to some of the channel parameters within the drop while keeping the channel parameters independent among drops. These time-variability features come from allowing the path delays and angles to drift at each simulation step within the drop caused by motion of the MS, which results in continuous evolution (in discrete steps smaller than a drop). The drifting of angles/delays is based on randomly generated distance from $\mathrm{Tx} / \mathrm{Rx}$ antennas to the closest scatters. [24].

- The WINNER II approach models the time-evolution of the parameters by controlling the position of clusters in the delay and angular domains using the following rules: (a) If the change in large-scale parameters (DS, AS) is small, a quasi-deterministic approach based on distance from IOs is applied at the initiation of the new drop, assuming that the small change in the large-scale parameters is caused by the same 
IOs as in previous drop. (b) If the change in local parameters is significant, then either the positions of the existing clusters are reassigned or their number is changed [24].

\subsection{Metrics to Detect Changes in Experimental Data}

Measurements show that mobile-channel characteristics change rapidly in space and time. To better characterize this change in radio channels, [28] introduced the idea of identifying changes in the phenomena that influence channel process, such as multipath propagation and obstruction shadowing. It is suggested that change points be used to identify the intervals in which the channel behaves in a similar way. Using this idea, the processes of changing conditions on radio channels have been investigated using different approaches. Several metrics have been proposed in the literature to measure the changes on radio channels. Some of these are explained in the following paragraphs.

\section{The Eigenvalues/Eigenvectors Metrics}

Viering [29] introduced a metric to measure a change in the covariance matrix of the channel impulse-response functions at different time intervals. The extracted covariance matrices represent the spatial signature of the channel at different time intervals. Each extracted covariance matrix is approximated using only the eigenvectors corresponding to its $F$ largest eigenvalues. To compare the low-rank approximation of covariance matrices existing in time intervals $T 1$ and $T 2$, the $F$-eigen-ratio metric was defined as the ratio between the traces of the two matrices. The $F$-eigen-ratio describes the change in the spatial structure of the channel during $T 1$ compared to $T 2$. To use this measure, the channel is assumed to be WSS during each time interval so that a sufficient number of channel matrices can be collected to estimate the covariance matrices.

Wallace [30] suggested describing the channel variations by quantifying the temporal variability of the channel eigenvalues and eigenvectors. The introduced metrics are: (a) eigenvalue level-crossing rate, which is the number of times that the power gain of each eigenmode drops below a certain threshold divided by the total distance travelled, (b) eigenvector angular deviation, which quantifies how quickly the transmit and receive 
eigenvectors rotate in complex multidimensional space, and (c) eigenvalue spread, which indicates the amount of multipath in the channel.

\section{The Correlation Matrix Distance Metric}

In [31 - 33] a correlation matrix distance metric was introduced for measuring changes in a channel by considering only the spatial domain of the channel. The correlation matrix distance is based on the inner product of two spatial matrices. This distance metric between $R_{t 1}$ and $R_{t 2}$ is defined as:

$$
D_{c o r}=1-\frac{\operatorname{tr}\left\{R_{t 1} R_{t 2}\right\}}{\left\|R_{t 1}\right\|_{F}\left\|R_{t 2}\right\|_{F}},
$$

where $\operatorname{tr}\{\cdots\}$ is the trace operator, and $\|\cdots\|_{F}$ denotes the Frobenius norm. $R_{t 1}$ and $R_{t 2}$ are the correlation matrices at intervals $t 1$, and $t 2$, respectively.

\section{The Spectral Similarity Metric}

Bultitude and Willink [28] introduced a metric to detect changes in radio-channel characteristics by considering changes in the AOA spectrum of the received signal. This metric is a distance measure between the spectral structures of the channel at different times. The categorization of wave numbers of incoming waves which correspond to specific AOAs is based on predefined non-overlapping sub-intervals. The sums of the powers within each sub-interval are compared to each other at different times using the following metric to define the difference between the spectra at time $T_{1}$ and $T_{2}$.

$$
D_{S S M}=1-\frac{\sum_{r=1}^{R}\left|S_{r T_{1}}-S_{r T_{2}}\right|}{\sum_{r}^{R} S_{r T_{1}}+\sum_{r}^{R} S_{r T_{2}}},
$$

where: $R$ is the total number of the spectrum non-overlapped sub-intervals. $S_{r T_{1}}$, and $S_{r T_{2}}$ denotes the sum of the powers in the rth sub-interval of the spectrum corresponding to the $T_{1}$ and $T_{2}$ time instant, respectively. 
The numerator of the second term in (2.3) is equal to zero for identical spectra, which makes the value of this measure unity. On the other hand, the measure equals zero for totally dissimilar spectra, in which the nominator would equal the sum of the two spectra under comparison.

\section{The Forward Prediction Metric}

Bultitude [34] took advantage of the availability of the amplitudes and phases of the impinging waves from the MUSIC algorithm under high SNR to reconstruct the plane-wave field along a trajectory and examine the correlation between the measured time series and forward predictions of the plane-wave reconstruction as a measure to detect changes. If the calculated cross-correlation coefficient was found to be less than a pre-selected threshold ( $90 \%$ is suggested), then a change in the channel was declared.

\subsection{Identifying Multipath Clusters}

Measurement results have shown that MPCs do not arrive uniformly in spatiotemporal space. Instead, energy is concentrated in clusters linked to reflections, scattering, and/or diffractions caused by IOs $[35,36]$. Clusters have been defined in the literature as accumulations of MPCs with similar spatio-temporal parameters (e.g., AOD, AOA and delay) that share the same long-term evolution (i.e., they stay intact over time) [28, 35, 37, 38]. Different MIMO channel models have employed the concept of multipath clusters intensively. However, quantifying and tracking the time-varying characteristics of clusters is still an open topic for research, and one that has been addressed by different researchers using different approaches.

Visual inspection was originally used to identify multipath clusters. However, this is a time-consuming process, especially for large data pools $[35,38,39]$. To make the identification of multipath clusters more practical, different automatic "clustering" algorithms have been introduced to group MPCs into clusters in spatio-temporal space. The different clustering algorithms can be categorized into two main groups: 
a) Joint-Delay-Angular Clustering: In this approach, MPCs are grouped into clusters using a total distance measure that describes how certain MPCs are close to (or farther from) each other jointly in both the delay domain and the angular domain. In these algorithms, the calculation of a single distance measure extracted from different quantities (e.g., delay, AOA and AOD) presents a major challenge due to the different nature, and hence the different units, associated with the delay and angular quantities involved. Two solutions have been suggested to solve this challenge: the use of parameter normalization [40], and the use of the Kernel density estimation technique $[37,41]$. Tracking the behaviour of clusters over time using such algorithms requires the identification of the clusters within each snapshot, then pairing the different clusters among different snapshots.

b) Sequential-Delay-Angular Clustering: In this approach, the clustering is performed first in the delay domain and then in the angular domain conditioned on the corresponding cluster delay [42, 43]. The sequential delay-angular clustering approach is preferable to the joint delay-angular approach for two reasons: 1) using the sequential delay-angular clustering approach simplifies the multidimensional clustering problem by transforming it into multiple sequential 1-D clustering problems, and 2) applying the sequential delay-angular clustering approach allows for the use of meaningful measures in each different dimension (i.e., delay vs. angular); and thus it eliminates the need to normalize two quantities with different natures in order to come up with a universal normalized value, which is needed for the multidimensional clustering algorithm.

\subsection{The Need for Measurement-Based Propagation Studies}

As explained in the previous sections, the modelling methodologies that are used in different channel models perform the simulation in drops in which the number of multipath clusters among consecutive drops might vary. In the current channel models, arbitrary assumptions are used to set the length of the drops, to control the change within each drop, and to describe the change in the parameters of multipath clusters during their 
active intervals. Hence, there is a need for more measurement-based studies to improve different capabilities of current channel models. For example,

1) Developing a new metric of change that considers the delay and angular characterstics of the channel. All the metrics that were mentioned in Section 2.6. tend to ignore consideration of the delay domain of the channel (i.e., they consider the narrowband channel), and some of them use the calculation of the channel covariance matrices, which requires the channel to be wide sense stationary during each time interval so that a sufficient number of channel matrices can be collected.

2) Developing a clustering algorithm that maintains the "sense" of the visual meaning of multipath clusters in such a way that the shapes of the identified clusters are almost identical to those of the clusters that would have resulted from using the visual inspection method.

3) Modelling the changes in the parameters of multipath clusters in the delay and angular domains during their active intervals.

Chapter 3 describes the propagation experiments and the data processing steps that were used to record and analyze channel-sounding data using a multiple antenna channel-sounder. In Chapter 4, the results from analyzing the measurement data were used to identify multipath clusters and to characterize the change over time in both the parameters of the identified multipath clusters, and the delay-angular characteristics of the channel. 


\section{CHAPTER 3:}

\section{SINGLE-DIRECTIONAL RADIO CHANNEL MEASUREMENTS AND DATA ANALYSIS}

In this chapter, the propagation experiments that were conducted in downtown Ottawa to record channel sounder data that were later processed to obtain estimates of the parameters of MPCs received at the CSMS are described. Section 3.1. describes the propagation experiments. Sections 3.2. and 3.3. report the Communications Research Center (CRC)-Chanprobe sounder specifications and the procedure used to extract the parameters of the MPCs, respectively. Section 3.4. reports an evaluation of the accuracy in estimating the channel parameters.

\subsection{Propagation Experiments}

Radio propagation data were recorded using the CRC-Chanprobe measurement system. The Chanprobe system includes a portable $2.25 \mathrm{GHz}$ Tx housed in a utility trailer that was parked near the curb on a selected busy street during weekday working hours. This Tx served to emulate a microcell BS, and transmitted a BPSK sounding signal modulated with a 255 chip, 50 Mchps PN sequence from a vertical quarter-wavelength monopole antenna supported at a height of about 6 metres above street level. A photograph is shown in Figure 3.1. 


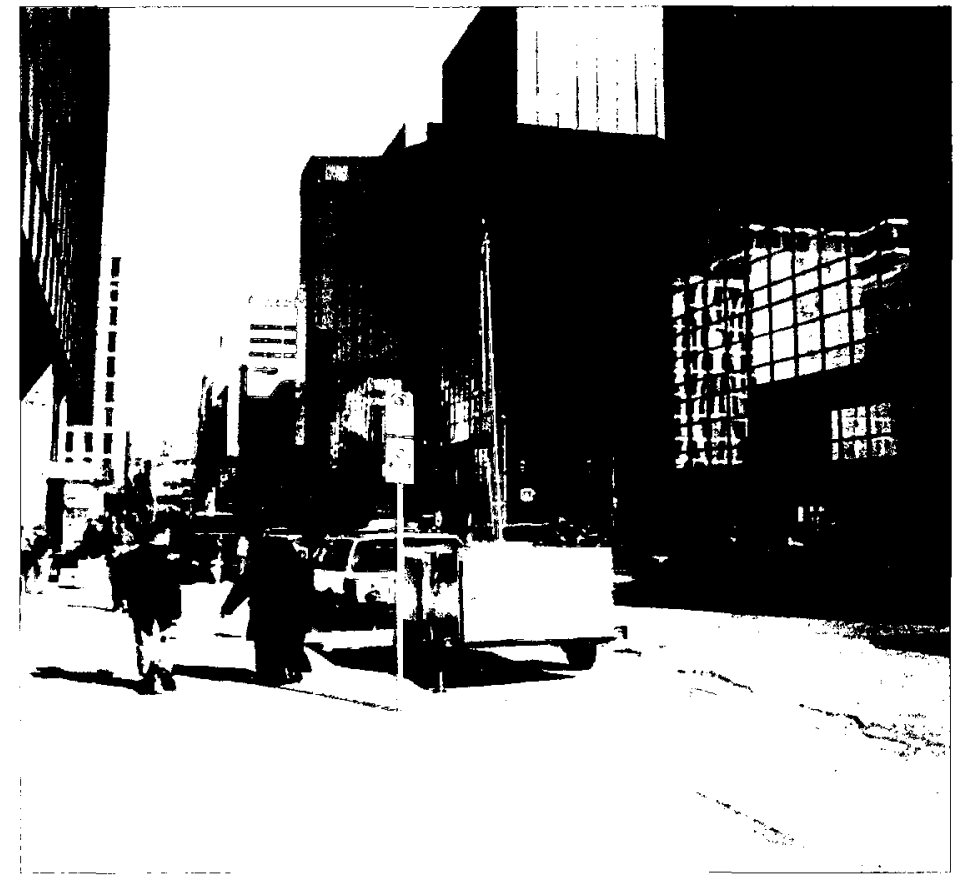

Figure. 3.1. Photograph showing the Tx configuration with the Tx monopole elevated to 6 metres above street level.

The CRC-Chanprobe sounder Rx system was mounted in a minivan (the white van ahead of the Tx trailer in Figure 3.1.), and received signals from the portable Tx via a 32 element Uniform Circular Array (UCA) (shown in Figure 3.2.), which was affixed to a wooden mount in the centre of the roof of the minivan. 


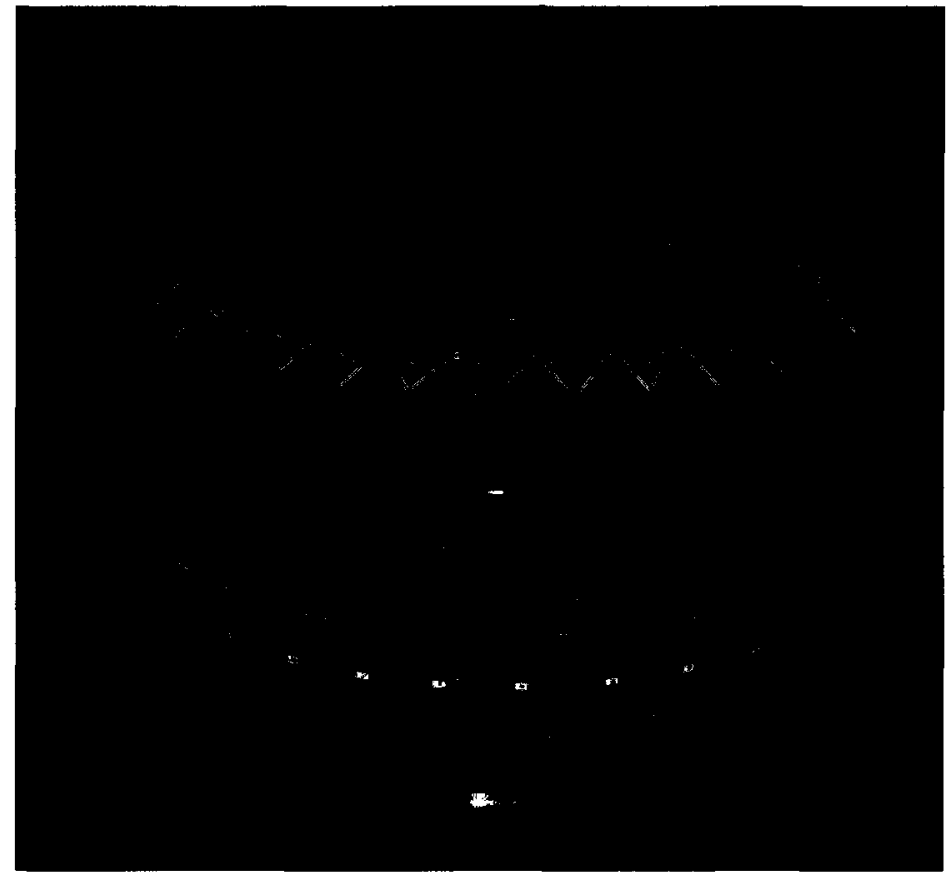

Figure. 3.2. Photograph of the 32 element UCA used for receiving during the reported experiments.

The $\mathrm{Rx}$ was connected sequentially to each antenna array element for 6 microseconds, the duration of 1 PN sequence length plus a settling interval, during which base-band in-phase (I) and quadra-phase (Q) samples of the received signal were recorded at a rate of $100 \mathrm{Msps}$ and stored in RAM. Snapshots, each comprising 32 single sequence lengths of data samples, were recorded every $4 \mathrm{~ms}$ as the minivan was driven over prescribed measurement runs at speeds between 10 and $20 \mathrm{kph}$. Each run lasted for between 10 and $20 \mathrm{~s}$. Figure 3.3. shows a Google photo of the area where the propagation data were recorded, including the location of the $\mathrm{Tx}$ and arrows showing some of the routes taken by the CSMS. 


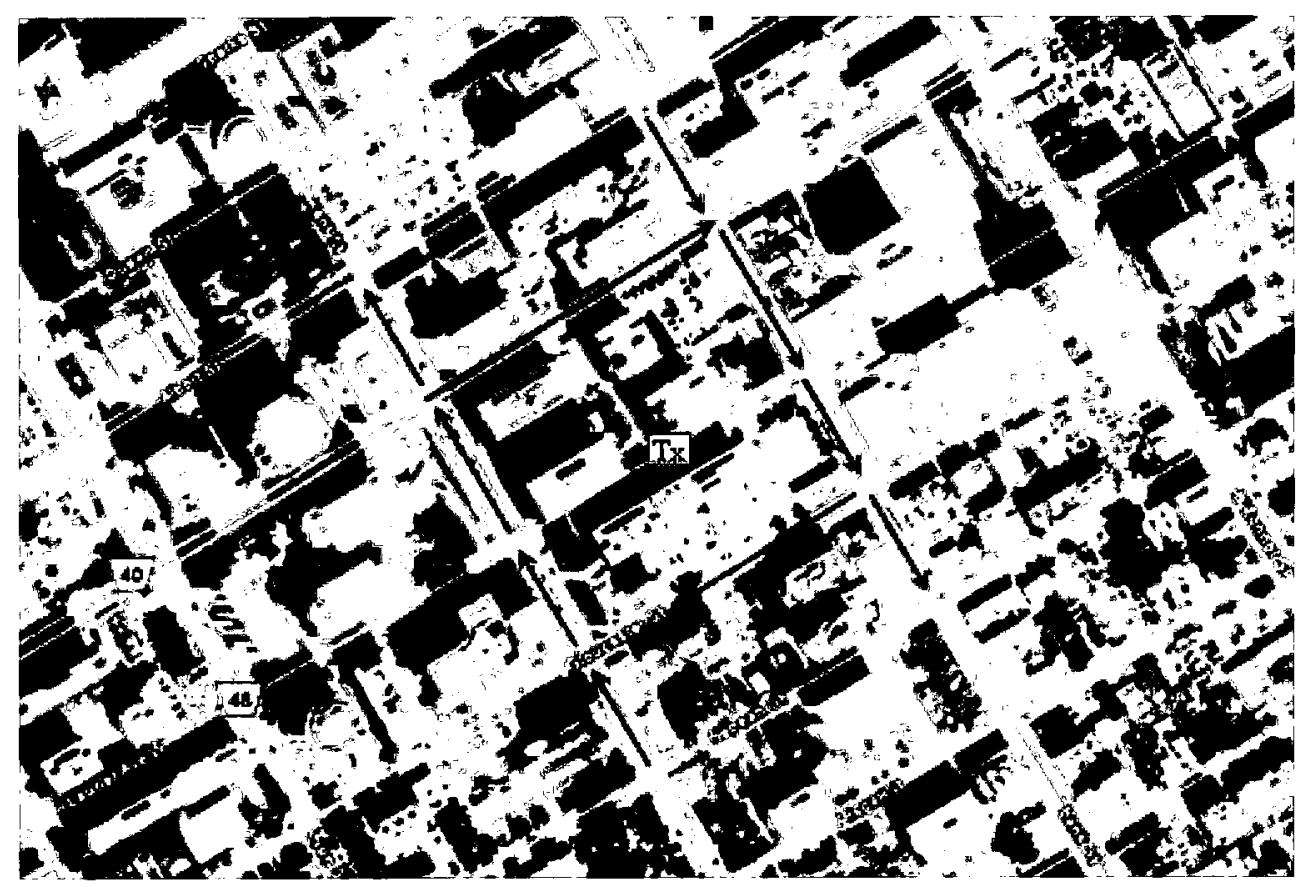

Figure 3.3. A view of the streets where propagation data were recorded illustrating the Tx location and some examples of the routes taken by the CSMS. 


\subsection{Sounder Specifications: CRC-Chanprobe}

The channel sounder that was used for the CRC propagation measurements is the fourth generation of a PN sounder design that was first implemented with $20 \mathrm{MHz}$ bandwidth at CRC in 1981. Its construction is breadboard style, with semi-rigid cables connecting various commercially available modules, such as phase-locked oscillators, power splitters, mixers, filter modules, and amplifiers. The breadboard-style construction is maintained so as to allow easy reconfiguration and recalibration for different measurement tasks, with different operating frequencies and different bandwidths, as required. Its PN sequence generator is a CRC implementation that can generate sequences of length between 127 and 1021 chips, and it can be clocked at rates up to 65 mchps. Both CRC-Chanprobe's $\mathrm{Tx}$ and its $\mathrm{Rx}$ have two RF sections with operating bandwidths centered on $2.25 \mathrm{GHz}$ and $5.8 \mathrm{GHz}$. Final downconversion is from IF to baseband via quadrature downconversion circuitry. The in-phase (I) and quadra-phase (Q) baseband outputs can each be sampled at rates up to $100 \mathrm{MSamples} / \mathrm{sec}$. More details about the Chanprobe's implementation can be found in [44].

At the time of the work reported herein, CRC-Chanprobe operated only in SIMO mode with a 32-element switched UCA that employs quarter-wavelength monopole antennas for the reception of vertically polarized waves. CRC-Chanprobe's operating characteristics are summarized in TABLE 3.1. 
TABLE 3.1: CRC-Chanprobe Operating Characteristics

\begin{tabular}{|c|c|}
\hline CRC-Chanprobe Property & Range of values \\
\hline RF bands & $0.95,2.25,(4.9), 5.8,30,40,60 \mathrm{GHz}^{[1]}$ \\
\hline Sustained measurement rate & 10,000 snapshots $/ \mathrm{S}^{[2]}$ \\
\hline Maximum cycle (snapshot) rate & 40,000 snapshots $/ S^{[3]}$ \\
\hline Chip rate & up to $50 \mathrm{Mchps}$ \\
\hline Useable code lengths & $127-1021$ chips (M-sequences) \\
\hline Number of measured radio links & 32 Switched $\mathrm{Rx}$ antennas, $1 \mathrm{Tx}$ antenna \\
\hline Measurement modes & $\begin{array}{l}\text { SISO, SIMO (double directional MIMO } \\
\text { beginning approx Sept./09) }\end{array}$ \\
\hline Receiver noise figure & better than $2 \mathrm{~dB}$ \\
\hline Baseband sampling rate & $100 \mathrm{MSamples} / \mathrm{s}$ \\
\hline Spurious IR free dynamic range: & $40 \mathrm{~dB}^{[4]}$ \\
\hline Transmitter output & $\begin{array}{l}\text { up to } 42 \mathrm{dBm} \text { at } 2.25 \mathrm{GHz} \text {, up to } 30 \mathrm{dBm} \text { at } \\
\text { other frequencies }\end{array}$ \\
\hline Control & Windows PC \\
\hline Post processing & MATLAB package \\
\hline Synchronisation & rubidium clock with stability of 10 e-11 \\
\hline $\begin{array}{l}\text { Minimum received power level }(20 \mathrm{~dB} \\
\text { MPSR) }\end{array}$ & $-89 \mathrm{dBm}$ \\
\hline $\begin{array}{l}\text { Linear dynamic range without pre- } \\
\text { attenuation }\end{array}$ & $-69 \mathrm{dBm}$ to $-89 \mathrm{dBm}$ with $20 \mathrm{~dB}$ MPSR \\
\hline Transmit antenna & $\begin{array}{l}\text { Vertical quarter-wavelength monopole, } \\
\text { with drooping radials }\end{array}$ \\
\hline Receive antenna & $\begin{array}{l}32 \text { element UCA of vertical quarter- } \\
\text { wavelength monopoles with drooping } \\
\text { radials }\end{array}$ \\
\hline
\end{tabular}

Note: Transfer rate specs are quoted assuming a single $\mathrm{Rx}$ channel, $50 \mathrm{Mchps} \mathrm{m}$-sequence, sequence length 255 chips, 2 samples per chip, 1 sequence length per snapshot.

[1] $0.95,4.9 \& 5.8 \mathrm{GHz}$ characteristics are SISO.

[2] Based on a verified average data acquisition rate of $\sim 20 \mathrm{Mbytes} / \mathrm{S}$ when logging data to hard disk in real time (needed for long measurement runs).

[3] Based on a verified average data acquisition rate of $\sim 80 \mathrm{Mbytes} / \mathrm{S}$ when not logging data to hard disk in real time (valid for short measurement runs).

[4] "Dynamic range" is a misnomer for this. It should be called Multipath Power Sensitivity Ratio (MPSR). The term "dynamic range" should be kept for the description of the power fading range over which the receiver operates linearly (i.e., Max RFinput Power-Min RF input power for linear operation: CRCChanprobe operates over the range $-69 \mathrm{dBm}$ to $-89 \mathrm{dBm}$, with $>20 \mathrm{~dB}$ MPSR). An attenuator after the Rx LNA can be automatically switched, however, to allow maximum received powers up to $-19 \mathrm{dBm}$. 


\subsection{Estimation of the Parameters of MPCs Received at the CSMS}

Post-processing of each snapshot of the data recorded at the CSMS during the propagation experiments began with the calculation of an Impulse Response Estimate (IRE) for each measured channel. This involved the cross-correlation of the single sequence length (510 samples) of complex baseband data recorded while the CSMS Rx was connected to each UCA antenna element with a previously recorded reference sequence of similar complex baseband data. The reference sequence was recorded when the channel sounder $\mathrm{Rx}$ was connected directly to the channel sounder Tx through a distortionless transmission line used to simulate an ideal radio channel. Since a sounding sequence with a reasonably high symbol rate was employed, such cross-correlations yield a good estimate of the impulse response, $h_{m}(\tau)$ of the radio link between the channel sounder transmit and the $m t h$ receive antennas [45]. Each of the 510 samples in each estimate of $h_{m}(\tau)$ represents the complex voltage that results from the reception of the vector sum of MPCs with delay $\tau$.

\section{Alignment of IRES}

Two levels of adjustments were applied to align the delays of the IREs as follows.

- Intra-snapshot alignment: In this step, the 32 IREs that result from sampling the signals from the 32 different elements at the UCA Rx were aligned. The Rx was connected sequentially to each antenna array element for 6 microseconds, which corresponds to 600 samples. Knowing that the used PN code has a length of 510 samples, this intra-snapshot alignment step was performed by circularly shifting each IRE by $i \times 90$ samples, where $i$ denotes the index of the $\mathrm{Rx}$ antenna element which takes the values of 0 to 31 .

- Inter-snapshots alignment: In this step, the IREs estimated from the different snapshots were aligned. The goal of this step was to align the delay of the IREs coming from different snapshots in such a way that if the channel were constant (i.e., if the CSMS and the operating environment were physically stationary), all the estimated IREs would be identical, with no relative delay shift among them. This alignment was achieved by compensating for the inter-snapshot relative 
delay shift that resulted from sampling the channel each $4 \mathrm{msec}$ at the $\mathrm{Rx}$. The inter-snapshot alignment was achieved by circularly shifting the 32 IREs from each snapshot (given that the intra-snapshot alignment had already been applied) by $s \times 160$ sample, where $s$ denotes the snapshot index. The 160 sample shift is derived from comparing the number of the total samples received in $4 \mathrm{msec}$ with the number of samples per PN code (number of receive samples in $4 \mathrm{msec}=$ $400,000=784 \times 510+160=784$ complete $\mathrm{PN}+160$ extra samples).

\section{Assumptions}

Throughout the analysis, the following two assumptions are adopted:

- Assumption 1: the plane-wave assumption, whereby a wave impinging on an antenna array can be approximated as having plane wavefront. This would be approximately true whenever the last interacting object on the radio path between the channel sounder Tx and Rx was far enough away from the Rx and Tx antennas so that, over the apertures of the antenna arrays, the curvature of the associated wavefront would have been negligible.

- Assumption 2: the narrowband assumption, whereby the signal bandwidth is assumed to be much smaller than the reciprocal of the time taken by a radio wave to propagate across the $\mathrm{Rx}$ antenna array. This assumption is only valid when the phase shift across the $\mathrm{Rx}$ antenna array is approximately the same for all frequency components of the received signal. Phase shifts would be nonnegligibly different only if the bandwidth of the sounding signal were to approach the reciprocal of the time taken for a wave in free space to travel across the array. Noting that the diameter of the CSMS UCA is only approximately $0.6 \mathrm{~m}$, it can be calculated that the condition described in the previous sentence would occur if the sounding signal bandwidth approached $500 \mathrm{MHz}$. However, the "rectangularequivalent" bandwidth of the channel sounding signal used during the work reported here was less than $100 \mathrm{MHz}$, so that the difference in phase shifts across the array for the frequency components at the upper and lower band edges would 
only be about $2.2 \mathrm{deg}$. It is considered that this is sufficiently small to render the reported analysis appropriate and valid.

Sections 3.3.1. and 3.3.2. explain the thresholding procedure used to eliminate the effect of the noise from the estimated channel impulse response functions and the steps used to extract the parameters of each MPC, respectively.

\subsubsection{Estimation of Channel Impulse Response Functions}

To eliminate the effect of noise from channel impulse response estimates, and to extract only valid multipath echoes, an adaptive thresholding algorithm was adopted [46], where the threshold was set so as to achieve a constant false-alarm rate independent of the SNR. This approach can be explained as follows:

For each cross-correlation estimate, the level of the background noise must be estimated. The complex parts of the $\mathrm{Rx}$ noise are assumed to be Gaussian, and its envelope is therefore assumed to be Rayleigh-distributed. In order to estimate the noise variance $\sigma_{N}^{2}$, and at the same time suppress the effect of the valid multipath echoes, $\sigma_{N}^{2}$ is evaluated based on the median of the samples that are certainly attributed to noise at large delays, where multipath arrivals are unlikely.

$$
\sigma_{N}=\sigma_{L D} / \sqrt{\ln 4} \approx 0.85 \sigma_{L D}
$$

where $\sigma_{L D}$ is the estimated median level of the recorded samples at large delays.

For each IRE, the constant false-alarm rate method, which is well known in radar, can be used by setting the thresholding level to $\zeta=\eta \cdot \sigma_{N}$, which makes the corresponding false alarm probability per sample

$$
P_{f}=\exp \left(-\eta^{2} / 2\right)
$$


For the measurement experiment explained in the previous sections, and taking into consideration that data recorded on 32 antenna elements are available at the $\mathrm{Rx}$, valid multipath echoes are declared, using the majority rule as follows. Valid multipath energy is declared in a specific impulse-response-delay resolution interval only if the signal in that interval exceeds the threshold in the IREs estimated from data at the outputs from 16 or more of the $32 \mathrm{Rx}$ antenna elements. Accordingly, the false-alarm probability per sample, calculated using the $32 \mathrm{Rx}$ elements, is calculated as:

$$
P_{F}=\sum_{i=16}^{32}\left(\begin{array}{c}
32 \\
i
\end{array}\right) P_{f}^{i}\left(1-P_{f}\right)^{32-i}
$$

Throughout the analyses reported in this thesis, $\eta=1.8797, P_{f}=0.1709$ and $P_{F}=1.9626 \times 10^{-5}$ are used, which corresponds to having one false alarm per 100 IREs. The IREs resulting from application of this thresholding procedure are processed further to extract the parameters of the MPCs, as explained in the following section.

\subsubsection{Channel Estimation using ISI-SAGE}

The initialization-search-improved space-alternating generalized expectationmaximization (ISI-SAGE) algorithm is a high-resolution estimation algorithm that performs joint estimation of the complex amplitude, delay, and AOA of superimposed incident signals. This algorithm is often used to analyze measured data recorded in different propagation environments because of its ability to perform the estimation using only one snapshot and the fact that, unlike other high resolution algorithms, it can be used to process data recorded with arbitrary array configurations. ISI-SAGE updates the parameters for each detected MPC sequentially by performing the search in several small data spaces instead of searching in the complete data space. This sequential parameter update makes ISI-SAGE more computationally efficient than the expectationmaximization method, while, at the same time, ISI-SAGE has a faster convergence rate than the expectation-maximization algorithm [47]. For example, if one is interested in 
estimating $\mathrm{K}$ parameters for each MPC, ISI-SAGE performs the search in $\mathrm{K}$ steps, and each step does the maximization in one-dimensional space; while the expectationmaximization algorithm performs the joint maximization in K-dimensional space. In work for this thesis project, the delay, then the AOA, and finally, the complex amplitude were estimated and updated for each MPC. The details of the ISI-SAGE procedure are explained in the subsequent paragraphs.

\section{Signal Model}

Let $u(t)$ be the complex baseband representation of the signal at the input to the Tx, and let the signal vector $y(t)=\left[y_{1}(t), \ldots, y_{M}(t)\right]^{T}$ be the signal at the output from the $\mathrm{Rx}$ where $M=32$ is the number of antenna elements at $\mathrm{Rx}$. Then, under assumptions 1 and 2 (Section 3.3.), the contribution of the $\ell$ th MPC to the signal vector $y(t)=\left[y_{1}(t), \ldots, y_{M}(t)\right]^{T}$ can be written as:

$$
s\left(t ; \Psi_{\ell}\right)=\alpha_{\ell} S V_{r x}^{1 \times M}\left(\varphi_{r x, \ell}, \theta_{r x, \ell}\right)^{T} u\left(t-\tau_{\ell}\right)
$$

and

$$
y(t)=\sum_{\ell=1}^{L} s\left(t ; \Psi_{\ell}\right)+\sqrt{\frac{N_{0}}{2}} w(t)
$$

where:

$\Psi_{\ell}=\left[\varphi_{t x, \ell}, \theta_{r x, \ell}, \tau_{\ell}, \alpha_{\ell}\right]$ is a vector with the parameters that characterize the $\ell$ th MPC: $\varphi_{r x, \ell}, \theta_{r x, \ell}, \tau_{\ell}, \alpha_{\ell}$ are, respectively, its azimuth AOA, elevation AOA, delay, and complex amplitude. Furthermore, $w(t)=\left[w_{1}(t), \ldots . ., w_{M}(t)\right]^{T}$ is an $M$ dimensional temporally and spatially white noise and $S V_{r x}\left(\varphi_{r x, \ell}, \theta_{r x, \ell}\right)$ represents the $1 \times M$ response column vector of the Rx array to a MPC arriving from angles $\left(\varphi_{r x, \ell}, \theta_{r x, \ell}\right)$, respectively. 
Estimating the channel parameters using ISI-SAGE consists of two steps [48, 49]. Step 1: Initialization-Search-Improved Procedure

A straightforward successive interference cancellation is used to initialize the parameters for each MPC. The parameter initialization is performed sequentially for the delay, the AOA (i.e., $\left.\left(\varphi_{r x, \ell}, \theta_{r x, \ell}\right)\right)$, and then the complex amplitude. The initialization algorithm is described in [48] as follows.

For $\ell=1, \ldots, L$, , initialization of the parameter estimates for the $\ell$ th MPC is performed as follows.

$$
\begin{gathered}
y^{\ell}(t)=y(t)-\sum_{\ell=1}^{\ell-1} s\left(t ; \hat{\Psi}_{\dot{\ell}}^{(0)}\right), \\
\hat{\tau}_{\ell}^{(0)}=\underset{\tau_{\ell}}{\arg \max }\left\{\sum_{m}\left|\int_{0}^{T_{s c}} u\left(t-\tau_{\ell}\right)^{*} y_{m}^{\ell}(t) d t\right|^{2}\right\}, \\
\left(\hat{\varphi}_{r x, \ell}^{(0)}, \hat{\theta}_{r x, \ell}^{(0)}\right)=\underset{\left(\phi_{r x, \ell} \theta_{r x, \ell}\right)}{\arg \max \left\{\left|\sum_{m} S V_{r x}\left(\varphi_{r x, \ell}, \theta_{r x, \ell}\right)[m]^{*} \int_{0}^{T_{s c}} u\left(t-\hat{\tau}_{\ell}^{(0)}\right)^{*} y_{m}^{\ell}(t) d t\right|\right\}} \\
\hat{\alpha}_{\ell}^{(0)}=\frac{\sum_{m} S V_{r x}\left(\hat{\varphi}_{r x, \ell}^{(0)}, \hat{\theta}_{r x, \ell}^{(0)}\right)[m]^{*} \int_{0}^{T_{s s}} u\left(t-\hat{\tau}_{\ell}^{(0)}\right)^{*} y_{m}^{\ell}(t) d t}{\left|S V_{r x}\left(\hat{\varphi}_{r x, \ell}^{(0)}, \hat{\theta}_{r x, \ell}^{(0)}\right)\right| P T_{s c}},
\end{gathered}
$$

where:

$s\left(t ; \hat{\Psi}_{\dot{\ell}}^{(0)}\right)$ is the contribution of the $\dot{\ell}$ th MPC with parameters $\hat{\Psi}_{\dot{\ell}}^{(0)}$ to $y(t)$. $\hat{\Psi}_{\dot{\ell}}^{(0)}=\left[\varphi_{r x, \ell}^{(0)}, \theta_{r x, \ell}^{(0)}, \tau_{\dot{\ell}}^{(0)}, \alpha_{\dot{\ell}}^{(0)}\right]$ is the vector of the initial estimates of the parameters of the $\ell$ th MPC. $T_{S C}$ is the time duration of the sounding signal. $y_{m}^{\ell}$ is the contribution of the $\ell$ th MPC that is received by the $m$ th antenna element, and $P$ is the power of $u(t)$.

The basic idea behind initializing the parameters of the MPCs using the successive-cancellation approach in (3.6)-(3.9) is explained as follows. To initialize the parameter vector of the $\ell$ th MPC (i.e., $\hat{\Psi}_{\ell}^{(0)}$ ), an estimate of the interference caused by 
the previously initialized MPCs is calculated and subtracted from the received signal, which yields $y^{\ell}(t)$ in (3.6). The initial values of the different parameters of the $\ell$ th MPC, i.e., $\tau_{\ell}^{(0)},\left(\varphi_{r x, \ell}^{(0)}, \theta_{r x, \ell}^{(0)}\right)$, and $\alpha_{\ell}^{(0)}$ are then successfully obtained based on the signal $y^{\ell}(t)$ as in (3.7)-(3.9). During this initialization step, the estimation in (3.8) is implemented using a so-called initialization-search-improved procedure, which uses an adaptive grid search with a grid size that decreases during the search procedure. In the analysis reported herein, the initialization step is carried on until a minimum of $95 \%$ of the power of $y(t)$ is extracted. The SAGE algorithm, combined with the initialization-search-improved procedure, is referred to as ISI-SAGE.

\section{Step 2: The SAGE procedure}

In this step, SAGE is applied to the outputs from the previous step to improve the parameter estimation. During each iteration, if the entries for the estimated vector $\hat{\Psi}_{\ell}$ associated with the $\ell$ th MPC are $\left\{\hat{\varphi}_{r x, \ell}^{\prime}, \hat{\theta}_{r x, \ell}^{\prime}, \hat{\tau}_{\ell}^{\prime}, \hat{\alpha}_{\ell}^{\prime}\right\}$, then the updated parameters $\left\{\hat{\varphi}_{r x, \ell}^{\prime \prime}, \hat{\theta}_{r x, \ell}^{\prime \prime}, \hat{\tau}_{\ell}^{\prime \prime}, \hat{\alpha}_{\ell}^{\prime \prime}\right\}$ are estimated as follows:

$$
\begin{gathered}
\hat{\tau}_{\ell}^{\prime \prime}=\underset{\tau_{\ell}}{\operatorname{argmax}}\left\{\sum_{m}\left|\int_{0}^{T_{s c}} u\left(t-\tau_{\ell}\right)^{*} \hat{x}_{\ell, m}(t) d t\right|^{2}\right\}, \\
\left(\hat{\varphi}_{r x, \ell}^{\prime \prime}, \hat{\theta}_{r x, \ell}^{n}\right)=\underset{\left(\phi_{r x, \ell}, \theta_{r x, \ell}\right)}{\arg \max }\left\{\left|\sum_{m} S V_{r x}\left(\varphi_{r x, \ell}, \theta_{r x, \ell}\right)[m]^{*} \int_{0}^{T_{s c}} u\left(t-\hat{\tau}_{\ell}^{\prime \prime}\right)^{*} \hat{x}_{\ell, m}(t) d t\right|\right\}, \\
\hat{\alpha}_{\ell}^{n}=\frac{\sum_{m} S V_{r x}\left(\hat{\varphi}_{r x, \ell}^{n}, \hat{\theta}_{r x, \ell}^{n}\right)[m]^{*} \int_{0}^{T_{s c}} u\left(t-\hat{\tau}_{\ell}^{\prime \prime}\right)^{*} \hat{x}_{\ell, m}(t) d t}{\left|S V_{r x}\left(\hat{\varphi}_{r x, \ell}^{n}, \hat{\theta}_{r x, \ell}^{\prime \prime}\right)\right| P T_{s c}},
\end{gathered}
$$

where $\hat{x}_{\ell, m}$ represents the contribution of the $\ell$ th $\mathrm{MPC}$ at the $m$ th receive element to $\hat{x}_{\ell}$.

In the above equations, the function $\hat{x}_{\ell}(t)$ is defined as:

$$
\hat{x}_{\ell}(t)=y(t)-\sum_{\ell=1, \ell \neq \ell}^{L} s\left(t ; \hat{\Psi}_{\ell}\right) .
$$


Equations (3.10)-(3.12) represent a single SAGE iteration step in which the entries of the parameter vector $\hat{\Psi}_{\ell}$ of the $\ell$ th MPC are re-estimated. A SAGE iteration cycle consists of $L$ SAGE iteration steps, where the parameter vector of each MPC is estimated once. The SAGE iterations are stopped if the improvement of the parameter estimation becomes smaller than a threshold, or if the number of iteration cycles reaches its maximum. In the analysis reported herein, $\varphi_{r x, \ell}, \theta_{r x, \ell}, \tau_{\ell}$ and,$\alpha_{\ell}$ were estimated for each MPC and 30 SAGE iteration cycles were used to improve the estimates.

\subsection{Evaluation of the Accuracy of Estimated Channel Parameters}

The accuracy of the parameters of MPCs estimated using ISI-SAGE was evaluated by comparing the $32 \times 510$ matrices of IREs resulting measurements with similar matrices of IREs constructed using the parameters estimated from ISI-SAGE as follows.

a) Wideband $32 \times 510$ matrices of IREs (i.e., $h_{\text {measured }}(t, \tau)$ ), each of which consists of the collection of the 32 IREs, were calculated (Section 3.3.1.) Then the ISI-SAGE was used to estimate the parameters of the MPCs (Section 3.3.2.)

b) The constructed channel matrices (i.e., $\left.h_{\text {constructed }}(t, \tau)\right)$ were calculated from the ISISAGE estimates of the MPCs parameters as summations of plane-waves as follows [50]:

First, plane-wave reconstruction was used to reconstruct the fields in the vicinity of the instantaneous locations of the $\mathrm{Rx}$ antennas (i.e. $\hat{y}(t)$ ), using the parameters of the MPCs resulting from application of the ISI-SAGE algorithm. Each estimates of the received signal is constructed as

$$
\hat{y}(t)=\sum_{\ell=1}^{L} s\left(t ; \hat{\Psi}_{\ell}\right),
$$

where $L$ is the total number of estimated MPCs, and $\hat{\Psi}_{\ell}$ is the parameter vector characterizing the $\ell$ th MPC that results from application of the ISI-SAGE algorithm. 
Second, the constructed channel matrices (i.e., $\left.h_{\text {constructed }}(t, \tau)\right)$ are calculated as the collection of the 32 IREs that result from the cross-correlation of each of the single sequence length (510 samples) of constructed complex baseband data with a copy of the transmitted PN code.

c) The reconstruction error for each snapshot is then calculated as:

$$
\text { Error }_{\text {reconstruction }}=\frac{\left\|h_{\text {measured }}(t, \tau)-h_{\text {constructed }}(t, \tau)\right\|_{F}^{2}}{\left\|h_{\text {measured }}(t, \tau)\right\|_{F}^{2}},
$$

where $\|\cdots\|_{F}$ is the Frobenius norm.

Figure 3.4. shows the Empirical Cumulative Distribution Function (ECDF) of the of Error $_{\text {reconstruction }}$ calculated from 35,000 snapshots analysed using ISI-SAGE. From Figure 3.4., it is found that Error $_{\text {reconstruction }}$ is less than $8 \%$ for $98 \%$ of the analyzed data.

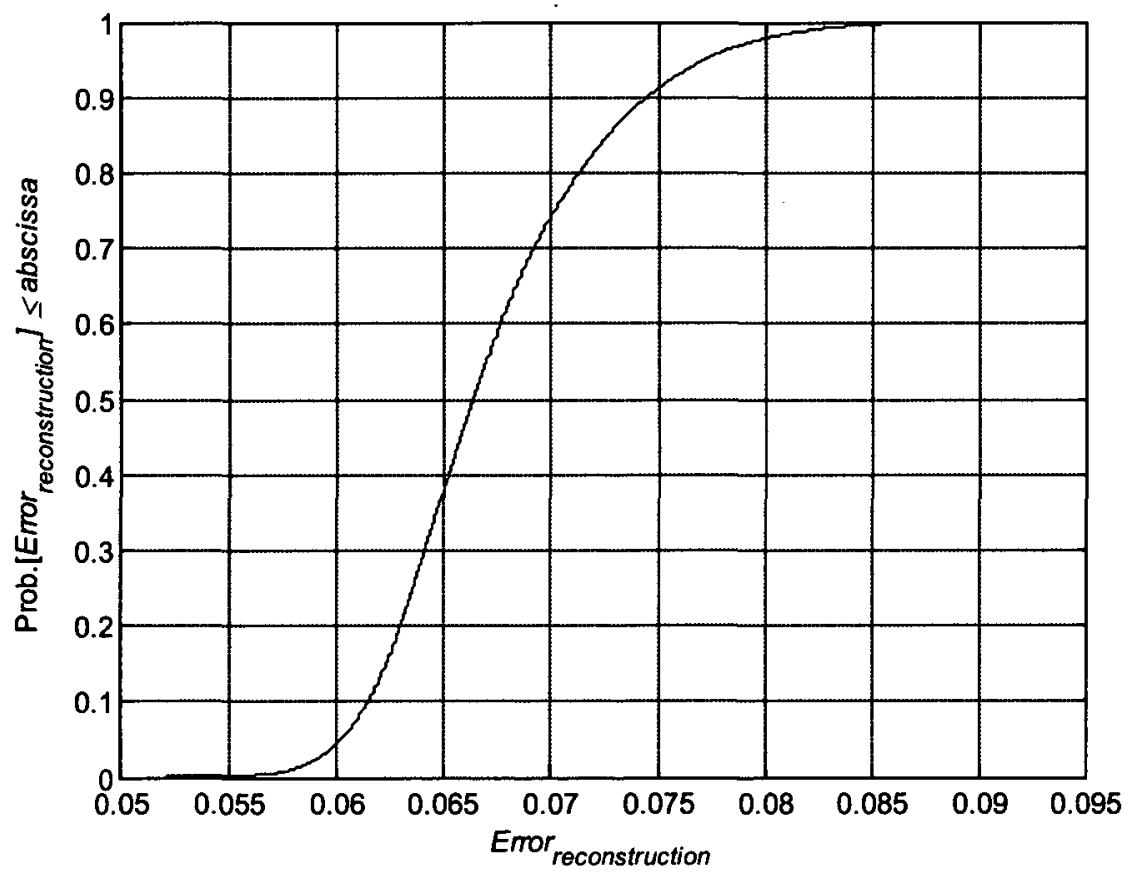

Figure 3.4. the ECDF of Error reconstruction calculated from 35,000 snapshots analysed using ISI-SAGE. 


\section{CHAPTER 4:}

\section{CLUSTER-BASED CHARACTERIZATION OF MICROCELLULAR MOBILE RADIO CHANNELS}

This chapter focuses on the use of the clustering nature of wireless channel as an effective tool for modelling the time evolution of wireless channels based on the analysis of measured radio propagation data. The specific goals of the work reported are (a) to estimate the boundaries of multipath clusters from propagation measurement data, and (b) to model the time-varying behaviour of identified clusters using stochastic, yet simple models. In Section 4.1, an algorithm for the identification of multipath clusters is introduced. In Section 4.2., the concept of Consistency Intervals (CI) to describe intervals between changes is reviewed. Section 4.3. reports results from analyzing propagation measurement data and includes: statistics on the number of clusters, statistics on the instantaneous values of different parameters of clusters, and the number of MPCs per cluster, as well as modelling of the time-varying behaviour of clusters.

\subsection{Identifying and Tracking Multipath Clusters}

In this section a new technique is proposed for the identification of multipath clusters in delay-angular space. The proposed clustering algorithm aims to: 1) avoid using a single-distance measure that is calculated by adding quantities with different natures such as delay, AOD, and AOA, which is typical when the Joint-Delay-Angular clustering approach is used, and 2) extract clusters of the MPCs in such a way that it is possible to track the change in their parameters over time, without the need for applying a pairing procedure.

As explained in Chapter 3, the outputs from application of the ISI-SAGE algorithm in the analysis of data from propagation experiments include: the complex amplitude, AOA, and the delay of each MPC in each snapshot. To identify and track multipath clusters, the ISI-SAGE outputs are processed as follows. 
1. Four seconds (during which 1000 snapshots of data were recorded during propagation experiments) was taken as the basic time interval for tracking and monitoring the behaviour of individual multipath clusters. For each interval of 1000 consecutive snapshots, the 2D delay-angular power spectrum was estimated as the average of the 2D delay-angular power profiles associated with each individual snapshot. It is considered that 4 seconds is long enough to get valuable information about the changes of the parameters of different multipath clusters during their active intervals. The result of this step is to have a single 2D spectrum describing the distribution of the power in the delay-angular domain for each group of 1000 consecutive snapshots. This $2 \mathrm{D}$ spectrum has a dimensionality of $510 \times 360$ (510 bins in the delay dimension with 10-nsec resolution, and 360 bins in the azimuth angular domain with 1-degree resolution). A normalized uniform circular averaging filter is applied for smoothing the resulting $2 \mathrm{D}$ spectrum before any further process. This filter is described as:

$$
h_{\text {circular }}=\frac{1}{49}\left[\begin{array}{ccccccccc}
0 & 0 & 0 & 0 & 1 & 0 & 0 & 0 & 0 \\
0 & 0 & 1 & 1 & 1 & 1 & 1 & 0 & 0 \\
0 & 1 & 1 & 1 & 1 & 1 & 1 & 1 & 0 \\
0 & 1 & 1 & 1 & 1 & 1 & 1 & 1 & 0 \\
1 & 1 & 1 & 1 & 1 & 1 & 1 & 1 & 1 \\
0 & 1 & 1 & 1 & 1 & 1 & 1 & 1 & 0 \\
0 & 1 & 1 & 1 & 1 & 1 & 1 & 1 & 0 \\
0 & 0 & 1 & 1 & 1 & 1 & 1 & 0 & 0 \\
0 & 0 & 0 & 0 & 1 & 0 & 0 & 0 & 0
\end{array}\right] .
$$

2. For each 2D spectrum resulting from Step 1, the proposed sequential 2D clustering algorithm (as will be described later during this section) is applied to identify the regions in the delay-angular domain where significant power exists in the received signal. Those regions will herein be called area multipath clusters (AMCs). The proposed sequential 2D clustering algorithm stops when at least 
$99 \%$ of the total power of the $2 \mathrm{D}$ spectrum is extracted. At the end of this step, the borders of the different AMCs and their corresponding delay-angular regions are identified.

3. At each snapshot, the MPCs with delay-angular coordinates that fall within the region of a specific AMC are defined as a single snapshot multipath cluster (SSMC). Each AMC could contain one or more SSMCs that exist sequentially in the specified region during the 1000 snapshots (i.e., in the same delay-angular region, only one SSMC can be active at a time, followed by a dormant interval, then another SSMC could be active in the same region, and so on). The borders of the delay-angular region of each specific $\mathrm{AMC}$ are used to determine the MPCs that belong to active SSMCs within each snapshot throughout the 1000snapshot interval. For example, if $\mathrm{R}_{i}$ represents the region of the ith identified $\mathrm{AMC}$, then all the MPCs that fall within the $\mathrm{R}_{i}$ delay-angular area in the sth snapshot would represent the corresponding SSMC at the sth snapshot. To make a clear distinction between the AMCs and the SSMCs, the following should be considered: a) an AMC represents a region on the 2D spectrum estimated from 1000 snapshots, where the time dimension (i.e, the snapshot index) is collapsed, because it is an average of information from 1000 snapshots, and b) a SSMC represents a region on the $2 \mathrm{D}$ power profile of a specific snapshot (i.e., the time dimension is preserved). In each snapshot, a pruning process is applied to each SSMC to remove the weak MPCs that are detached from its delay centroid or angular centroid. This is done by deleting the group of MPCs that are close to the borders in such a way that $95 \%$ of the power in each SSMC is preserved. The remaining MPCs in each SSMC are then used to estimate the parameters that characterize each SSMC within each snapshot. Subsequently, these parameters are used to track the time-varying behaviour of the individual SSMCs within the 1000-snapshot interval. The parameters that are used to characterize each SSMC in each snapshot are: its power, its rms DS and its rms AS. In addition, the length of the active interval, which is defined as the number of consecutive snapshots in which a SSMC exists, is calculated. 
4. A thresholding procedure is applied to eliminate very weak SSMCs. In each snapshot, each SSMC with power less than 3\% (i.e. down by about $-15 \mathrm{~dB}$ ) of that belonging to the strongest SSMC is ignored.

Figure 4.1 illustrates the steps that are used to identify and track the time-varying behaviour of SSMCs throughout a 1000-snapshot interval. In Figure 4.1.a., the proposed 2D clustering algorithm is applied to the delay-angular spectrum that is calculated from 1000 snapshots. The 2D spectrum is represented by the square, and the three ellipses symbolize the borders of the regions of three AMCs that are identified using the proposed 2D clustering algorithm. In Figure 4.1.b., the coordinates of the regions of the identified AMCs are used to track each SSMC and its different parameters throughout the 1000 snapshots. In the region of the first identified AMC, one SSMC, $\mathrm{C} 1$, exists for the entire [x1:x7] interval. The region of the second identified AMC is dormant during the [x1:x3] interval, followed by an active interval [x4:x7], which is occupied by $\mathrm{C} 2$. Finally, the third identified $\mathrm{AMC}$ has an active interval $[\mathrm{x} 1: \mathrm{x} 2]$ occupied by $\mathrm{C} 3$, followed by a dormant interval [x3:x5]; then it is occupied by $C 4$, which is active during the [x6:x7] interval. 


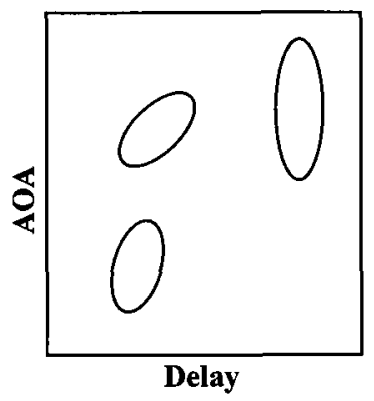

(a)

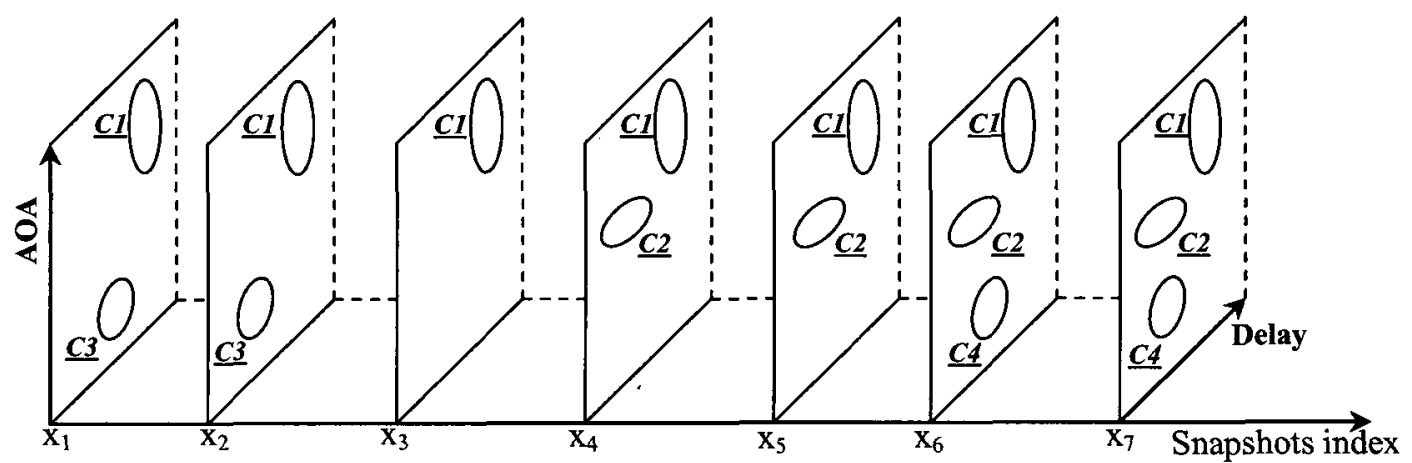

(b)

Figure 4.1. Illustration of the steps used to track the different parameters of multipath clusters within a 1000-snapshot interval. (a) applying the proposed 2D clustering algorithm, and (b) tracking SSMCs. 


\section{Sequential 2D Clustering}

Let $S$ be the normalized (unity power) 2D delay-angular spectrum that results from averaging 1000 snapshots. The AMCs can be identified using Algorithm 4.1 below:

\section{Algorithm 4.1.}

Step 1: Find the location of the maximum value of $S$ and declare it to be the peak of the $\mathrm{AMC}$ to be identified.

Step 2: Apply the 2D valley detection algorithm (explained below) to determine the borders of the targeted AMC.

Step 3: Delete the components of the identified AMC from $S$.

Step 4: If the remaining power of $S \leq 0.01$, stop. Otherwise, go to Step 1 .

\section{The 2D Valley Detection Algorithm}

To estimate the boundaries of an AMC (in the delay-angular domain), the 2D valley-detection algorithm was developed during this thesis project. This algorithm is an extension of the 1D valley-detection algorithm introduced in [51]. As a first step, the original 1D valley-detection algorithm is explained, and then its new $2 \mathrm{D}$ extension is introduced.

\section{A- 1D Valley Detection by Use of One-Sided Difference Operators}

Given a 1D discrete waveform $x_{1}, \ldots . ., x_{N}$ consider the point $x_{k}$ and let

$$
\begin{aligned}
& a_{i}^{(k)}=\sum_{v=k-2 i+1}^{k-i} x_{v}, \\
& b_{i}^{(k)}=\sum_{v=k-i+1}^{k} x_{v}, \\
& c_{i}^{(k)}=\sum_{v=k}^{k+i-1} x_{v}, \\
& d_{i}^{(k)}=\sum_{v=k+i}^{k+2 i-1} x_{v} .
\end{aligned}
$$

The quantities $a_{i}^{(k)}, b_{i}^{(k)}, c_{i}^{(k)}$, and $d_{i}^{(k)}$ are only defined if $k-2 i+1>0$ and $k+2 i-i<N$. Define different operators to calculate the left-hand and right-hand 
differences using windows with different sizes $i$, and define the left-hand and right-hand differences of size $i$ at $x_{k}$ as:

$$
\begin{aligned}
& l_{i}^{(k)}=\frac{1}{i}\left(b_{i}^{(k)}-a_{i}^{(k)}\right), \\
& r_{i}^{(k)}=\frac{1}{i}\left(c_{i}^{(k)}-d_{i}^{(k)}\right) .
\end{aligned}
$$

A prominent valley should give a negative value for both $l_{i}^{(k)}$ and $r_{i}^{(k)}$ that does not change for a large number of sizes $\left(i^{\prime} s\right)$. In the proposed algorithm, a point $x_{k}$ is considered to be a prominent valley if it results in negative values of both $l_{i}^{(k)}$ and $r_{i}^{(k)}$ for $i=1,2,3,4$, and 5 .

\section{B- New Extension to 2D}

The above-described 1D algorithm can be extended to the 2D (i.e., the delayangular) case as follows.

Step 1: Find the peak of the candidate $A M C$ : let $P k=\left(x_{P k}, y_{P k}\right)$ be the projection of the maximum value of $S$ on the 2D delay-angular plane (which is denoted as $X Y$ ) and declare it as the location of the peak of the candidate AMC.

Step 2: Extract $1 D$ waveforms: let $v$ be the vector on the delay-angular plane that starts at the point $\mathrm{Pk}$ in the $\varphi$ direction, then define the waveform $W_{v}$ which consists of all the points of $S$ such that their projection on the delay-angular plane intersects with the vector $v$ such that:

$$
W_{v}=\left\{p: p \in S, p \perp X Y=(x, y), \tan ^{-1}\left(\left[x-x_{P k}\right] /\left[y-y_{P k}\right]\right)=\varphi\right\},
$$

where $\perp$ denotes the projection operation.

Let the value of $\varphi$ be changed in such a way that the vector $v$ scans the whole azimuth plane in steps of 1 degree; and let a new waveform $W_{v}$ be defined for 
each new direction that the vector $v$ takes. Applying this process results in 360 $1 \mathrm{D}$ waveforms $W_{v}$.

Step 3: Detection of the valleys of each waveform $W_{v}$ : the 1D valley-detection algorithm is applied to detect the valley point in each of the 1D waveforms $W_{v}$. For each waveform, the search for the valley starts from the point $P k$ in the associated $\varphi$ direction and stops when the first valley is found, which results in a total of 360 valley points. These 360 points might be not unique due to the delay-angular resolution of the sounding system where the number of the points on the border of the AMCs in most cases are less than 360.

Step 4: Determining the borders of the $A M C: 1)$ The group of points that represent a unique set of points are selected from the resulting 360 non-unique points. 2) The borders of the identified AMC are defined by applying a 5-tap uniform smoothing filter to those unique points. See Figures 4.2. and 4.3. 


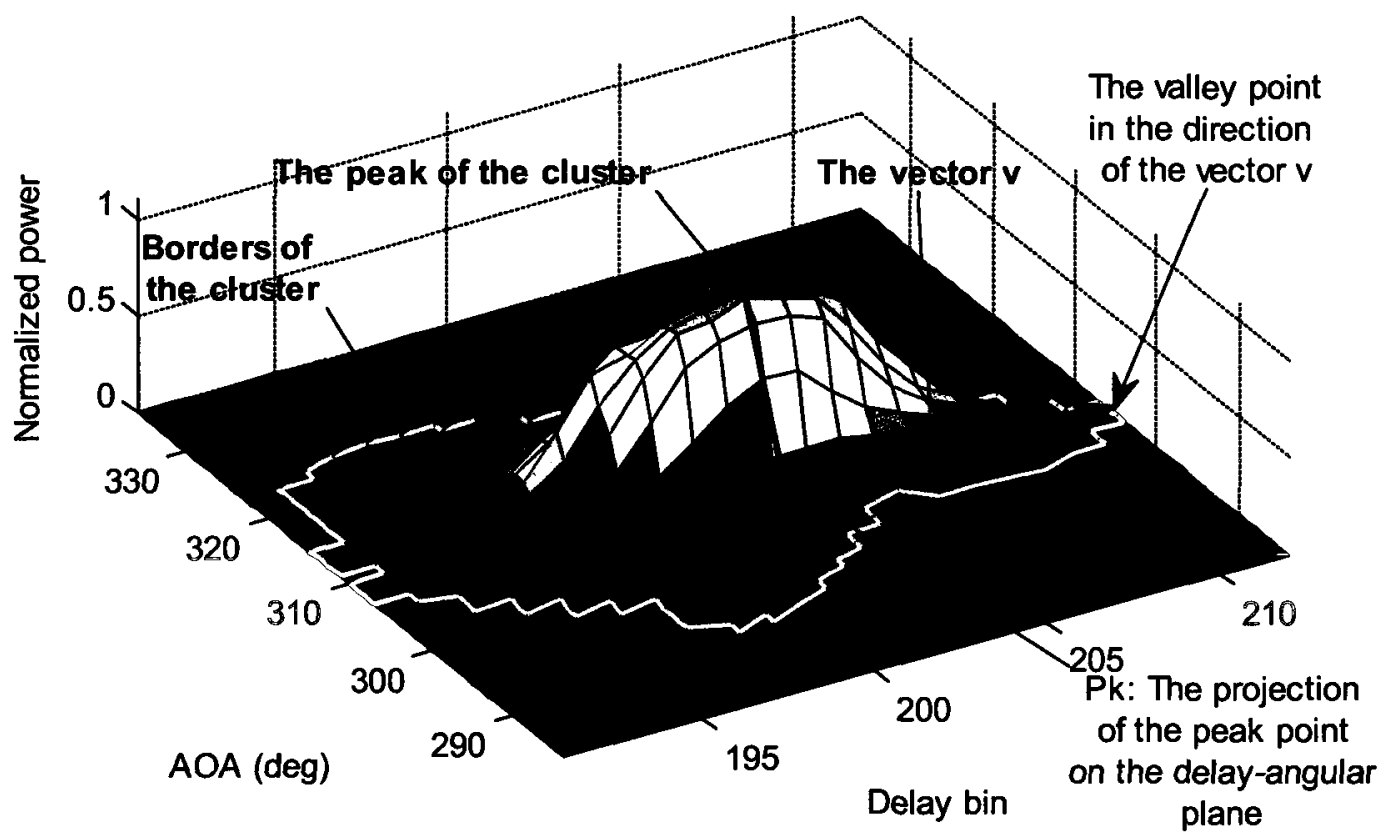

(a)

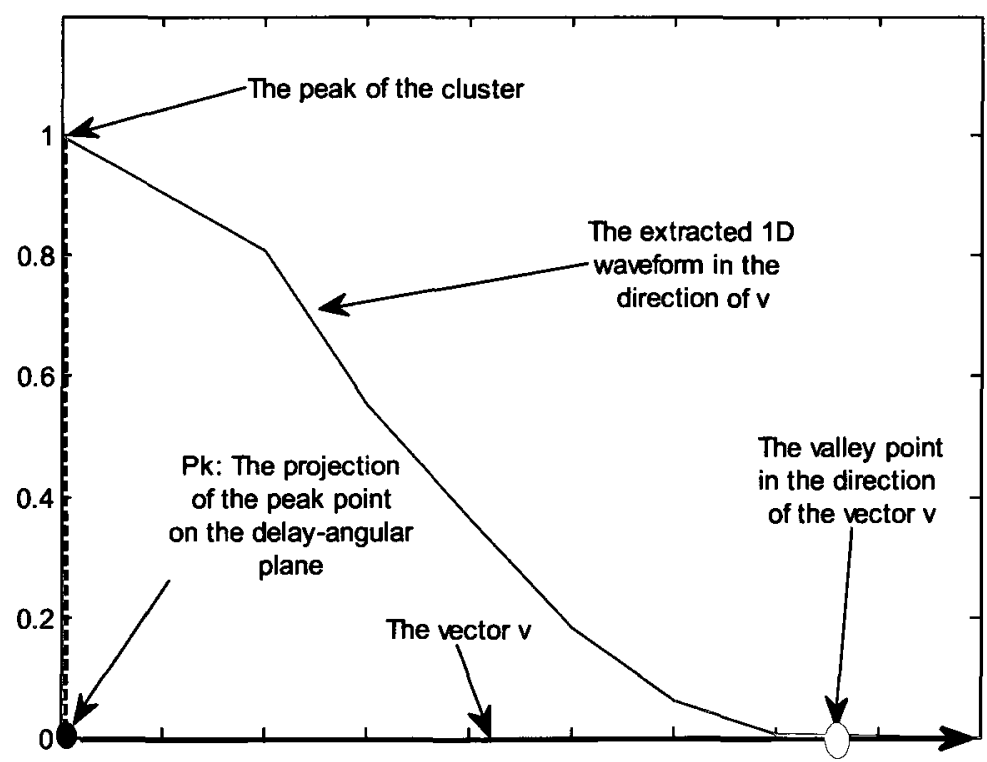

(b)

Figure 4.2. The sequential $2 \mathrm{D}$ clustering algorithm. (a) $3 \mathrm{D}$ illustration of an AMC where a vector $v$ represents the direction of interest that is used to extract a 1D waveform. (b) a plot of the extracted waveform in the direction of $v$ with the identified valley point in this direction. 


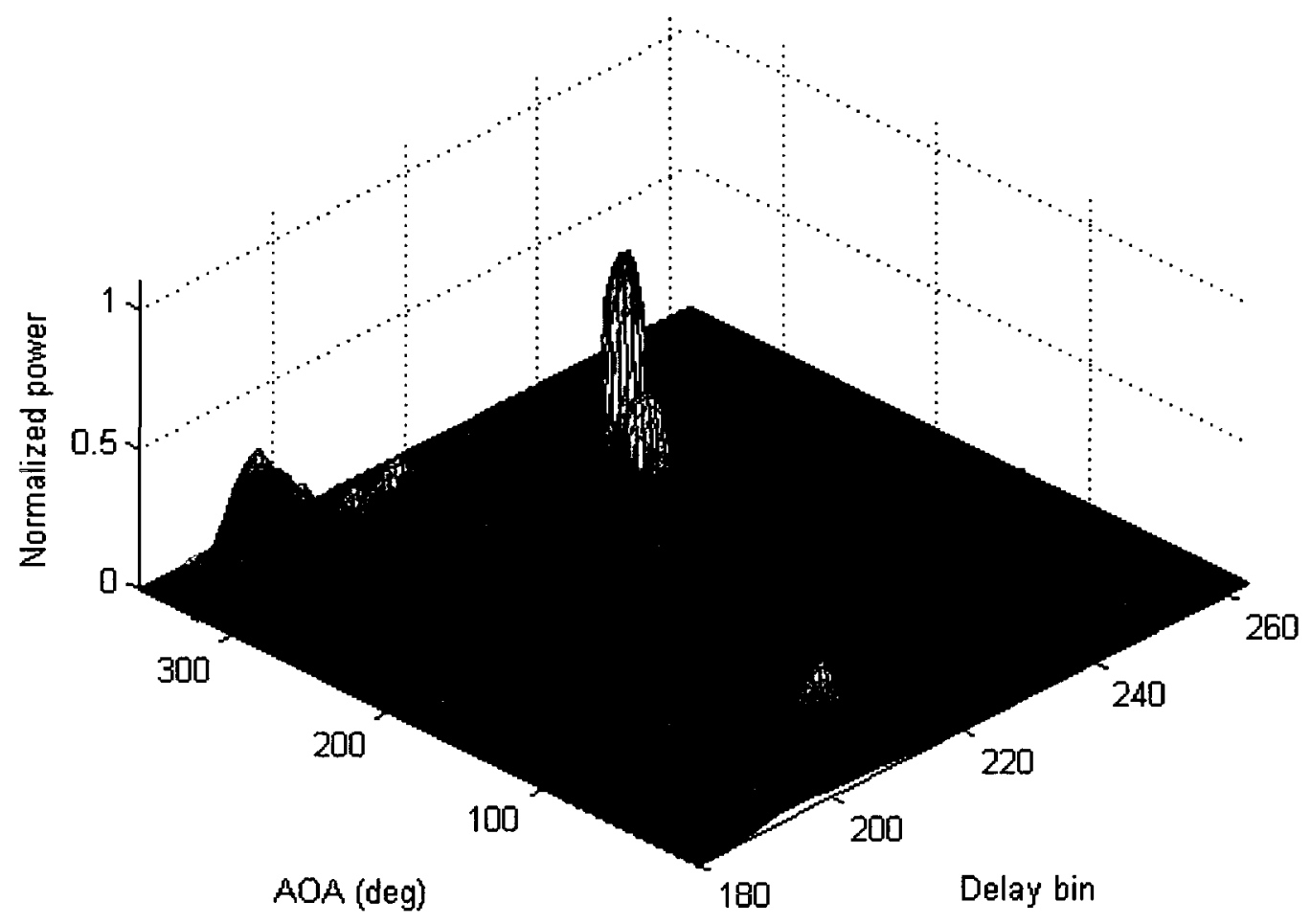

Figure 4.3. Examples of identified AMCs resulted from analyzing propagation data using the proposed $2 \mathrm{D}$ clustering algorithm. 


\subsection{Identifying Consistency Intervals}

Let $C_{1}, C_{2}, \ldots, C_{K}$ be the AMCs identified using the sequential $2 \mathrm{D}$ clustering algorithm (Section 4.1), and let $R_{1}, R_{2}, \ldots, R_{K}$ be the regions that contain the delay-angular coordinates of all the points of the detected AMCs in the delay-angular domain. Let $P_{k, s}$ be the summation of the powers of the MPCs in the sth snapshot that fall within $R_{k}$. That is

$$
P_{k, s}=\sum_{\substack{i \\\left(\tau_{i}, \varphi_{r, i}\right) \in R_{k}}}\left|\alpha_{i}\right|^{2},
$$

where $\alpha_{i}, \tau_{i}$, and $\varphi_{r x, i}$ are the complex amplitude, the delay, and the azimuth AOA of $M P C_{i}$, and $M P C_{i}$ belongs to the sth snapshot.

Define the difference between snapshot $s_{x}$ and snapshot $s_{y}$ as

$$
D_{s_{x}, s_{y}}=\sum_{k=1}^{K}\left|P_{k, s_{x}}-P_{k, s_{y}}\right| \text {, }
$$

where $|.$.$| denotes the absolute value.$

A CI that starts at snapshot $s_{x_{0}}$ and ends at snapshot $s_{X}$ (i.e., $C I_{\left[x_{0}: X\right]}$ ) is defined as the interval of all the consecutive snapshots, such that their differences with respect to the first snapshot $s_{x_{0}}$, calculated as in (4.6), are less than a specified threshold $(\gamma)$. This can be written as:

$$
C I_{\left[x_{0}: X\right]}=\left\{s_{x}: D_{s_{0}, s_{x}}<\gamma, \forall x_{0} \leq x \leq X\right\}
$$

Applying the abovementioned criterion guarantees that the summation of the change in the power distribution in the delay-angular domain, for all snapshots within a CI, does not exceed $\gamma$ when it is compared to the first snapshot $s_{x_{0}}$. 


\subsection{Results}

The proposed 2D clustering algorithm was applied to a total of 35,000 snapshots that were recorded on 16 NLOS measurement routes surrounding a Tx on Laurier Avenue between Bank and Kent Streets in downtown Ottawa. Each AMC that was extracted from a 2D spectrum, estimated as explained in Section 4.1., represents a delay-angular region that contains a significant amount of power. Taking into consideration that each $2 \mathrm{D}$ spectrum is calculated using data from 1000 consecutive snapshots, the identified regions in the delay-angular domain might not be occupied with significant power throughout all the 1000 snapshots. In other words, when considering the time dimension (i.e., the dimension calibrated by snapshot indices which go from 1 to 1000) for each AMC, it was found that each AMC might contain one or more SSMC that appears and disappears sequentially in the same delay-angular region. The active interval of a SSMC is defined as the number of consecutive snapshots in which a specific SSMC exists. The interval between snapshots where two active SSMC in the same region are identified will be called a dormant interval. The parameters of the identified SSMC were estimated, including: 1) the number of SSMCs in each snapshot, and 2) the power, the intra-cluster DS, and the intra-cluster AS of each SSMC in each snapshot ${ }^{1}$. For each snapshot, the amplitudes of the MPCs are adjusted such that the total power reflected by the identified MPCs in each snapshot is unity. Hence, the reported power of each SSMC can be considered to be a normalised power.

\subsubsection{Number of SSMCs}

Figure 4.4. shows an estimate of the CDF for the number of SSMC per snapshot. It is clear that the number of SSMC, with very large probability, does not exceed 20 . It should be noted that, for each snapshot, only SSMC with power equal or exceeding $3 \%$ of the power of the strongest SSMC are considered. The median number of SSMC equals 8 , and the probability of having more than 10,15 and $20 \mathrm{SSMC}$ is found to be $35 \%, 10 \%$ and $3 \%$, respectively. To model the number of SSMCs, the time series assembled using

\footnotetext{
${ }^{1}$ No attempt has been made to model the delay and angular centroids of SSMCs.
} 
consecutive values for the number of SSMCs per snapshot was systematically sampled with different interval lengths until the time series of the resulting samples passed the Wald-Wolfowitz runs test, which is a non-parametric statistical test that checks a randomness hypothesis for a two-valued sequence. This sampling step resulted in a time series of 120 random values, each of which represent a value for the number of SSMCs at a specific time instance. It was found that the number of SSMCs can be modelled as a Gamma distribution with parameters $\mathrm{a}=4.27$, and $\mathrm{b}=2.10$. This model passes the Kolmogorov-Smirnov (KS) goodness of fit test at the 5\% significance level. The CDF resulting from this model is shown in Figure 4.4, along with the CDF estimated from the experimental data.

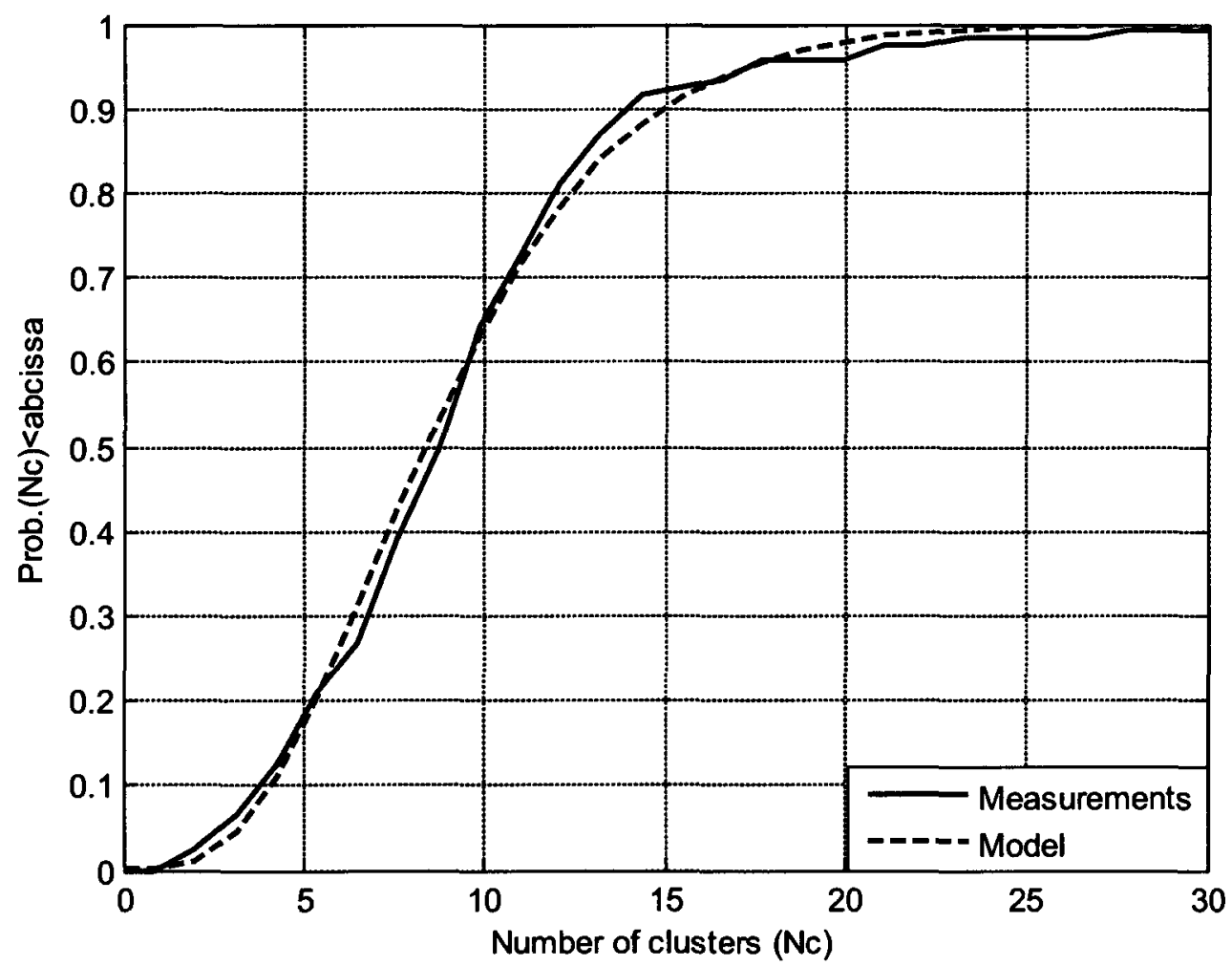

Figure 4.4. CDF of the number of SSMCs. "Blue" color is measurements, and "Red" color is the best-fit Gamma model which has parameters $a=4.27$, and $b=2.10$. 


\subsubsection{Classification of SSMC}

The identified SSMCs were classified into three different groups according to their mean power: Group A has a normalized mean power greater than 0.20 , Group B has a normalized mean power between 0.10 and 0.20 , and Group $C$ has a normalized mean power less than 0.10. It was found that strong SSMCs experience longer active intervals than weak SSMCs. The SSMCs in Groups A, and B are active for entire 1000-snapshot intervals $55 \%$ and $16 \%$ of the time, respectively, while no SSMC from Group C was found to be active for an entire 1000-snapshot interval. Figure 4.5. shows the length of the active intervals that are less than 1000 snapshots extracted for the three groups. TABLE 4.1 shows the parameters that are used to model the length of the active intervals of the multipath clusters for the three groups. 

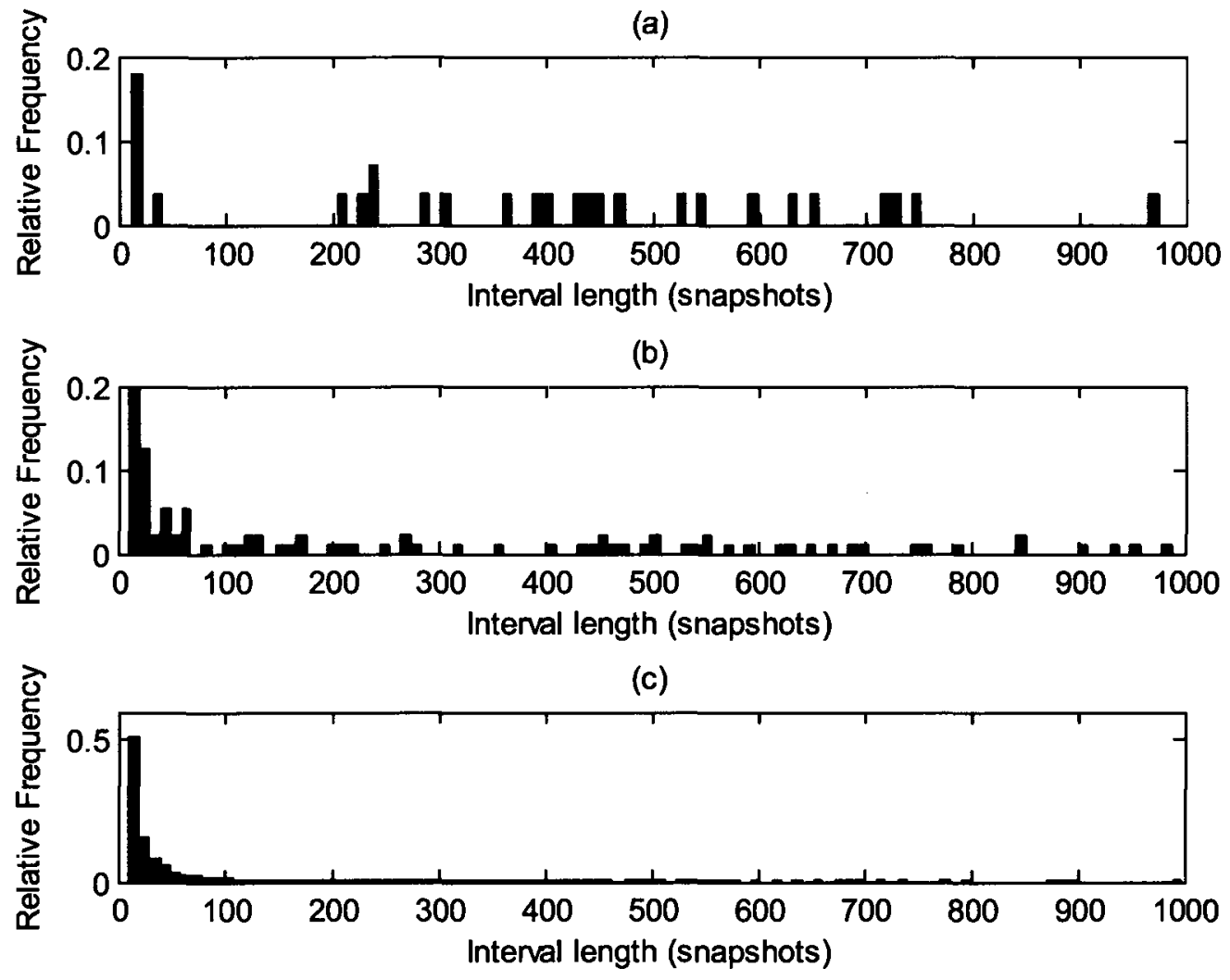

Figure 4.5. Lengths of active intervals that are less than 1000-snapshots long for different groups of SSMCs identified from measurement data. (a) Group A, (b) Group B, and (c) Group C.

TABLE 4.1 Parameters used to Model the Length of the Active intervals for the SSMCs belonging to Different Groups.

\begin{tabular}{|c|c|c|c|}
\hline \multirow[b]{2}{*}{ Group } & \multirow[b]{2}{*}{$\begin{array}{l}\text { Normalized } \\
\text { power }\end{array}$} & \multicolumn{2}{|c|}{ Modelling the length of active intervals } \\
\hline & & $\begin{array}{l}\text { Prob. of being active for the } \\
\text { entire } 1000 \text { snapshots }\end{array}$ & $\begin{array}{l}\text { Model for the length of the active } \\
\text { interval given that their length is } \\
\text { less than } 1000 \text { snapshots }\end{array}$ \\
\hline A & $>0.20$ & $55 \%$ & $\begin{array}{l}\text { Uniformly distributed between } 1 \\
\text { and } 1000 \text { snapshots }\end{array}$ \\
\hline B & $\begin{array}{c}\geq 0.10 \text { and } \\
\leq 0.20\end{array}$ & $16 \%$ & $\begin{array}{l}\text { Exponentially distributed with } \\
\text { parameter }=258 \text { snapshots }\end{array}$ \\
\hline $\mathrm{C}$ & $<0.10$ & $0 \%$ & $\begin{array}{l}\text { Exponentially distributed with } \\
\text { parameter }=50 \text { snapshots }\end{array}$ \\
\hline
\end{tabular}


Different combinations of the three SSMC groups can exist in different detected AMCs, where the strong AMCs have a higher probability of containing strong SSMCs (i.e., Group A). TABLE 4.2. shows the different combinations of the SSMCs of Groups $\mathrm{A}, \mathrm{B}$, and $\mathrm{C}$ that were found in the strongest $20 \mathrm{AMC}$ groups that are identified in each of the measured 2D delay-angular spectrums. For example, $\mathrm{AMC}$ group $\mathrm{ID}=1$ represents the group of AMCs results from collecting the strongest AMC that is identified in each of the measured 2D delay-angular spectrums.

TABLE 4.2. The Percentage of SSMCs from Groups $A, B$, and $C$ in Different AMC groups Identified in Measured Data.

\begin{tabular}{|c|c|c|c|}
\hline AMC group ID & $\begin{array}{c}\text { Prob. of having a } \\
\text { SSMC from Group } \\
\text { A }\end{array}$ & $\begin{array}{c}\text { Prob. of having a } \\
\text { SSMC from Group } \\
\text { B }\end{array}$ & $\begin{array}{c}\text { Prob. of having a } \\
\text { SSMC from Group } \\
\text { C }\end{array}$ \\
\hline 1 & $50 \%$ & $15 \%$ & $35 \%$ \\
\hline $2-3$ & $5 \%$ & $15 \%$ & $80 \%$ \\
\hline $4-8$ & $0 \%$ & $5 \%$ & $95 \%$ \\
\hline$>9$ & $0 \%$ & $0 \%$ & $100 \%$ \\
\hline
\end{tabular}

The strong AMCs tend to have shorter dormant intervals; the weaker AMCs tend to have longer dormant intervals. It was found that the length of the dormant intervals for each AMC group can be modelled as having an exponential distribution with the parameters shown in TABLE 4.3. 
TABLE 4.3. The Values of Parameters of the Exponential Distribution used to Model the Length of Dormant Intervals for Different AMC group

\begin{tabular}{|c|c|}
\hline AMC group ID & $\begin{array}{c}\text { Exponential Distribution Parameter } \\
\text { (snapshots) }\end{array}$ \\
\hline 1 & 7 \\
\hline 2 & 5 \\
\hline 3 & 4 \\
\hline 4 & 5 \\
\hline 5 & 8 \\
\hline 6 & 7 \\
\hline 7 & 9 \\
\hline 8 & 10 \\
\hline 9 & 10 \\
\hline 10 & 9 \\
\hline 11 & 13 \\
\hline 12 & 12 \\
\hline 13 & 19 \\
\hline 14 & 13 \\
\hline 15 & 20 \\
\hline 16 & 32 \\
\hline 17 & 39 \\
\hline 18 & 28 \\
\hline 19 & 36 \\
\hline 20 & 39 \\
\hline
\end{tabular}

\subsubsection{Number of MPCs}

Figure 4.6. shows the ECDF of the number of MPCs per SSMC for Group A, B, and $\mathrm{C}$ during their active intervals. Group $\mathrm{A}$, which contains the strongest SSMCs, has more MPCs per cluster, with a median equal to 11 . The median of the number of MPCs in Group B and C are 8 and 7, respectively. 


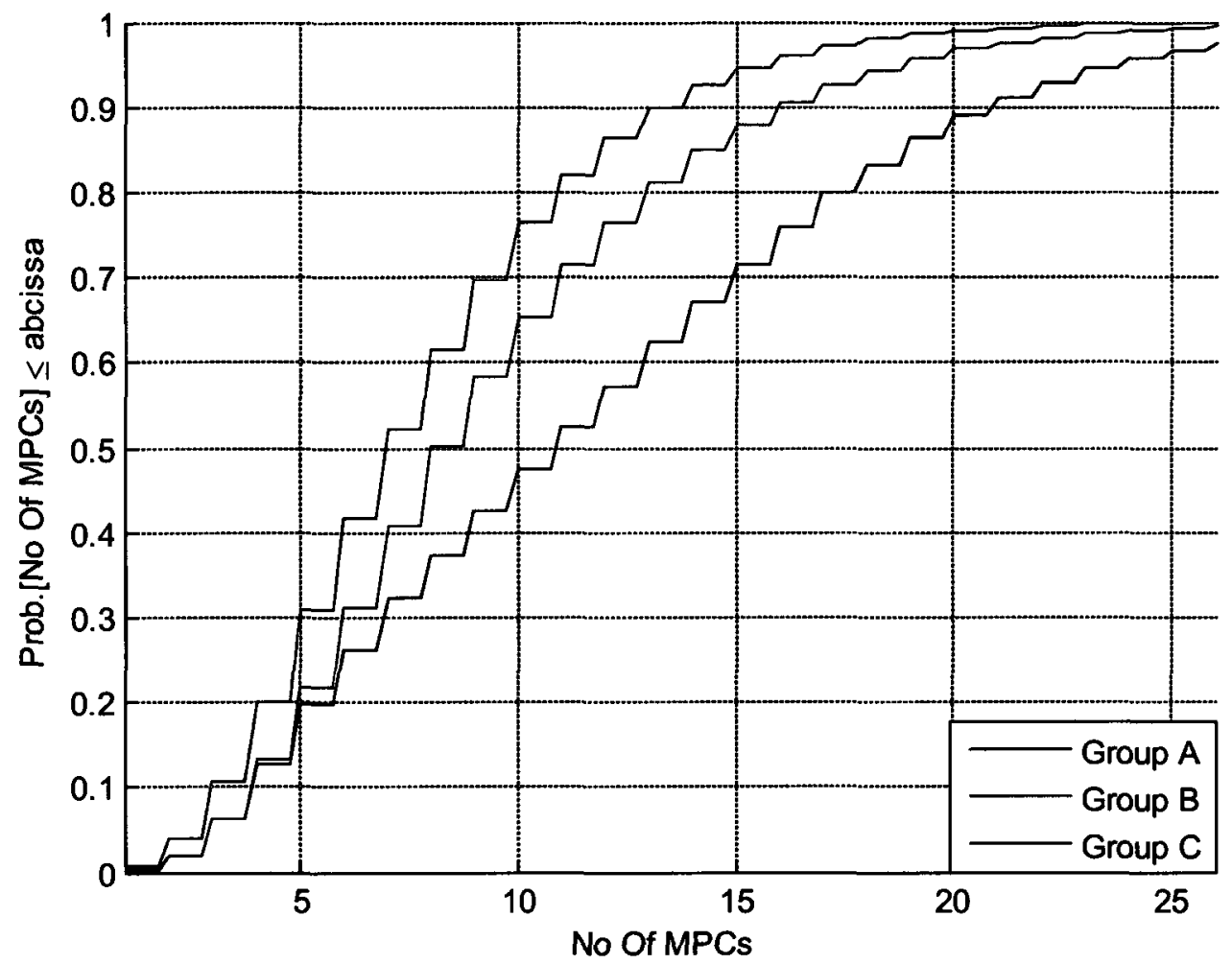

Figure 4.6. ECDFs for the number of MPCs per SSMC for Groups A, B, and C.

\subsubsection{Modelling the Power of SSMCs}

It was found that the power of SSMCs in each of the three groups can be modelled using the Generalized Extreme Value (GEV) distribution. Several researchers have previously reported that this distribution can be used to model fading in wireless channels better than well-known models such as the Nakagami, Rice and Rayleigh models [52 56].

The GEV distribution can be mathematically represented as [57]:

$$
\operatorname{Pr}[X \leq x]=\exp \left\{-\left[1+\xi\left(\frac{x-\mu}{\sigma}\right)\right]_{+}^{-1 / \xi}\right\},
$$

where the notation $a_{+}$stands for $a$ if $a>0$ and zero otherwise, and where $\sigma>0$ and $\mu, \xi \in R$. 
When $\xi>0$ or $\xi<0$, the distribution in (4.8) is referred to as either the Fréchet distribution or the Weibull distribution, respectively. When $\xi \rightarrow \infty$ or $\xi \rightarrow-\infty$, the distribution in (4.8) is referred to as the Gumbel distribution.

The three parameters used to characterize the GEV distribution are the shape $(\xi)$, the scale $(\sigma)$, and the location $(\mu)$ parameters. The shape parameter $(\xi)$ governs the behaviour of the distribution at its tail; the scale parameter $(\sigma)$ controls the spread (i.e., stretching/shrinking) of the distribution; and the location parameter $(\mu)$ determines the location (or the shift) of the distribution on the abscissa.

The GEV distribution was applied herein to model the distribution of the power of the SSMCs within each of the three Groups. In Figure 4.7., subplots a, b, and c, show the estimates for the distribution of the power of SSMCs and their best fit GEV distribution models for Groups A, B, and C, respectively. It was found that a time series of the powers of the SSMCs can be modelled using the GEV distribution, and that this model passed the KS goodness of fit test at the 5\% significance level. An explanation of using the GEV distribution to model the power of SSMCs can be given as follows. From the definitions of the GEV distribution, if a data set (A) is generated from some distribution, and a new data set ( $\left.A^{\prime}\right)$ is created by including the values of $A$ that are bounded by a maximum or a minimum threshold, then resulting data set (A') can only be described by the GEV distribution. Given that the categorization of the SSMCs is based on a maximum or a minimum threshold selection, then the GEV represents a logical model for the power of the different groups of SSMCs. TABLE 4.4. lists the values of the shape $(\xi)$, the scale $(\sigma)$, and the location $(\mu)$ parameters used to model the powers of the SSMCs in the different groups. In TABLE 4.4., note that, in all cases, the shape parameter ( $\xi$ ) has small absolute values $(<0.40)$. This indicates that these distributions are of either the Fréchettype or the Weibull-type. 
TABLE 4.4. The Values of the Shape $(\xi)$, the Scale $(\sigma)$, and the Location ( $\mu$ ) Parameters Used to Model the Powers of SSMCs in Groups A, B, And C.

\begin{tabular}{|c|c|c|c|}
\hline Group & \multicolumn{3}{|c|}{ Power } \\
\cline { 2 - 4 } & $\xi$ & $\sigma$ & $\mu$ \\
\hline A & -0.10 & 0.16 & 0.29 \\
\hline B & 0.13 & 0.07 & 0.11 \\
\hline C & 0.38 & 0.02 & 0.03 \\
\hline
\end{tabular}




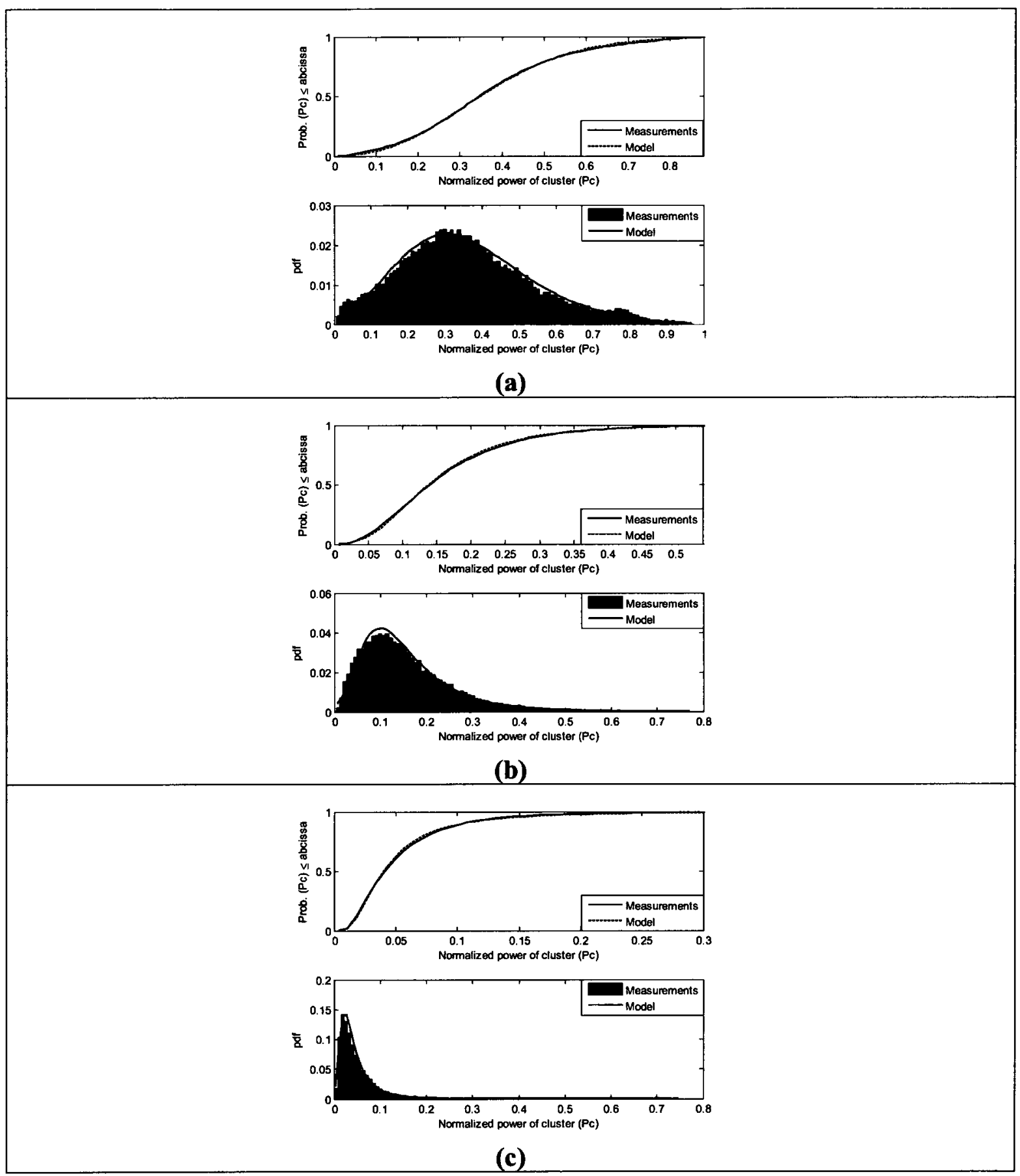

Figure 4.7. The ECDF and epdf of the powers of the SSMCs in the different groups with their GEV best-fit models. (a) Group A, (b) Group B, and (c) Group C. 


\subsubsection{Modelling SSMC Dynamics}

The goal of the work reported in this section was to represent the change in the parameters of the different SSMCs using simple, yet representative, stochastic models. The changes in the power of each cluster, intra-cluster rms DS, and intra-cluster rms AS are modelled in such a way that its time-varying behaviour is stochastically described.

It was found that the magnitude of the changes in power (i.e., the step size of the change) of SSMCs between consecutive snapshots has an exponential distribution. It was also found that: 1) the time series of the sizes of those steps are uncorrelated (the correlation was found to be $<0.20$ at a lag equal to one snapshot), and 2) the probability of the step sign (i.e, direction) being positive (i.e, increment step) is equal to the probability that it is negative (i.e., decrement step). The step sizes of the change in power for the SSMCs in groups A, B, and C were modelled as exponential random variables, with parameters equal to $0.0122,0.0069$, and 0.0036 , respectively.

Using the abovementioned properties of the instantaneous powers of identified SSMCs and the increment/decrement steps, Algorithm 4.2, below, was developed to generate power values for different SSMCs in such a way that the generated power values and their time-varying behaviour mimic the behaviour of the SSMCs in the three groups. 
A: $\left\{\begin{array}{l}L=\text { length of current interval } \\ \text { Expiration Counter }=0, \text { Cntr }=1, J=1 \\ \text { Initialize histogram }\end{array}\right.$

B: $\quad$ while $(J<L)$

C: $\quad\left\{\begin{array}{l}\text { if }(J==1) \\ v=\text { random value } \\ \text { else } \\ v=D A T A(\text { Cntr-1)+ random step } \\ \text { Increment ExpirationCounter } \\ \text { End }\end{array}\right.$

D: $\quad\left\{\begin{array}{l}\text { if (ExpirationCounter reaches maximum value) } \\ v=\text { random value } \\ \text { End }\end{array}\right.$

E: $\{$ Adjust the histogram according to the value of $v$

F: $\quad\left\{\begin{array}{l}\text { if the histogram condit } \\ D A T A(\text { Cntr })=v \\ \text { Increment Cntr } \\ \text { Increment } J \\ \text { Expiration Counter }=0 \\ \text { else } \\ v=\text { random value } \\ \text { end }\end{array}\right.$

end_while

Algorithm 4.2. Algorithm for generating power values for clusters for an interval with length $L$ snapshots in such a way that: a) the distribution of the generated power values follows a specific distribution, and b) the change in the powers of SSMCs between consecutive snapshots is modelled as a step that has an exponential distribution for its size with equal probability of being either an increment or a decrement. 
The steps in Algorithm 4.2 describe how to generate power values for a certain SSMC during an active interval of length $L$ snapshots in such a way that: a) the distribution of the generated series of values follows a specific distribution, and b) the magnitude of the change of powers between consecutive snapshots is modelled as an exponential distribution, with equal probability of being either an increment or a decrement. The main attributes of Algorithm 4.2. are explained as follows:

- Random initialization: At certain points in Algorithm 4.2. (in Steps C, D, and F), random initialization of the value for SSMC power is required. This initialization is done by selecting a random value between 0 and the 99 th percentile of the associated GEV distribution, where the parameters of the GEV distribution for different SSMCs are taken from TABLE 4.4.

- Generating the increment/decrement steps: Step $\mathrm{C}$ requires the generation of a value for the step that describes the change in power of the SSMC. The step size is picked from an exponential distribution with a parameter corresponding to the group to which the SSMC belongs. Each step has an equal probability of having a positive sign (i.e., increment) or a negative sign (i.e., decrement).

- Adherence to the GEV distribution: Steps E and F requires that the distribution of the generated power values follows the GEV distribution that characterizes the parameters corresponding to each SSMC. This can be done by generating a histogram representing the desired distribution; each bin of this histogram is used to count the number of values $v$ that are generated by the algorithm and that fall within a certain range. Each accepted generated value $v$ is followed by reducing the count in the corresponding bin by 1 (Step E). If a certain bin contains a count of zero, no more values $v$ in this range are accepted; instead, the value of $v$ is forced to be reset randomly (Step F). In this work, the number of values used to generate the histogram is $10 \%$ more than the number of required values (i.e., the length of the interval $L$ ); 100 bins were used to characterize the distribution. 
Figure 4.8. shows the autocorrelation function resulting from analysis of the measurement data and the data simulated using Algorithm 4.2. From this figure, it is clear that the autocorrelation functions of the power values of SSMCs from measurement and simulations have the same behaviour for the different three groups. The mismatch between the autocorrelation function of the measurement data and that of the simulated data contributes to the statistics of the CIs, which will be explained in the next section.

It has also been found that the time-varying behaviour of the delay and angular parameters can be modelled using an Autoregressive (AR) model. Following [58], let $g_{i, l, k}$ denote the time series of the parameter under consideration during the $l t h$ active interval of the $k$ th SSMC, where $i$ can take one of the values 1,2 to denote the rms DS, or the rms AS, respectively.

Let $\widetilde{g}_{i, l, k}$ be the normalized version of $g_{i, l, k}$, calculated as:

$$
\widetilde{g}_{i, l, k}=\left(g_{i, l, k}-\mu_{g_{i, l, k}}\right) / \sigma_{g_{i, l, k}},
$$

where $\mu_{g_{i, l, k}}$, and $\sigma_{g_{i, l, k}}$ are the mean and the standard deviation of $g_{i, l, k}$

Let $\widetilde{G}_{i, k}$ be the collection of all the time series over all intervals of the ith parameter associated with the $k t h$ SSMC. $\widetilde{G}_{i, k}$ can be modelled as an 20th-order AR process. The Yule-Walker algorithm was used to find the coefficients of the corresponding AR model. 


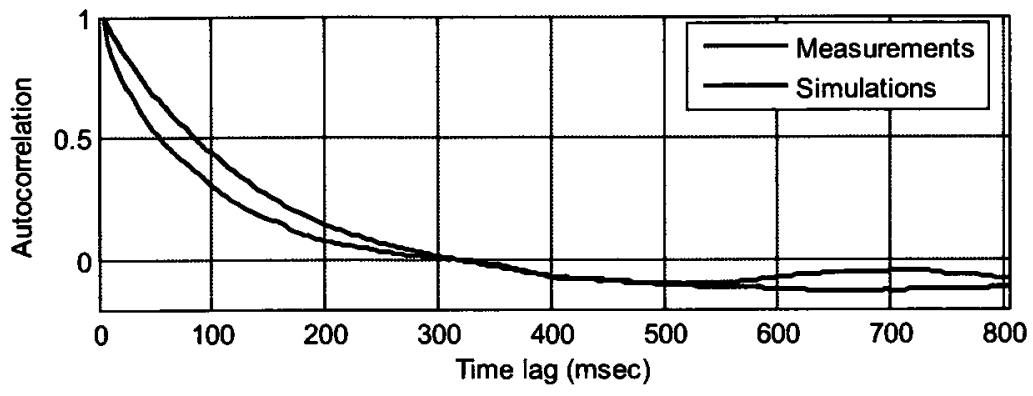

(a)

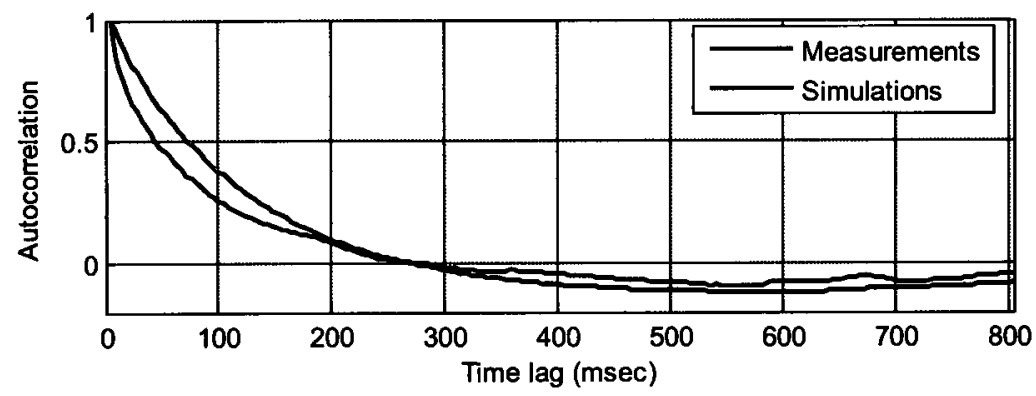

(b)

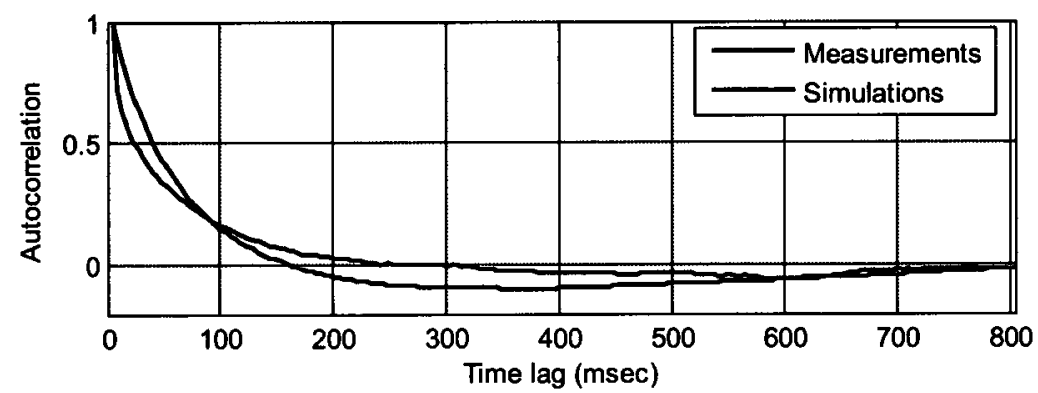

(c)

Figure 4.8. Comparison of the autocorrelation functions of the power values of SSMCs from measurements and simulations in different groups. (a) Group A, (b) Group B and (c) Group C. 
Figure 4.9. shows the autocorrelation function of the process resulting from normalizing results from analysis of the measured data according to (4.9), and the autocorrelation function generated using the $\mathrm{AR}$ models. It is clear from the figures that both the measured data and the results from the proposed model have very similar timevarying behaviours for the parameters under consideration.

\subsubsection{Comparing the Statistics of Consistency Intervals Identified in Times Series of Measured Data and in Simulation Results}

The models that were reported in the previous sections of this report were applied to simulate: the length of the active intervals of SSMCs, the change in power of the SSMCs during their active intervals, and the lengths of dormant intervals. The simulation was done using 50 trials, each one representing a time series of 1000 snapshots. During the simulations, each trial snapshot was made to contain 20 AMCs, each of which could contain different types of SSMCs. The information in TABLE 4.2. was used to select the types of the SSMCs for each AMC, and that in TABLE 4.1. was used to simulate the length of the intervals for the SSMCs. TABLE 4.3. was used to simulate the dormant intervals between SSMCs.

The means of CIs that were identified in simulated time series data are compared to those of CIs identified in the measured data. The range of the threshold (explained in Section 4.2.) extends from 0.3 , which corresponds to detecting a very small change, to 0.9 , which corresponds to detecting only major changes. TABLE 4.5 . lists the means of the CIs identified in simulated time series data and in the measured data at different thresholds, where the errors in the means of the CIs were found to range from $8 \mathrm{~ms}$. to 32 ms. 


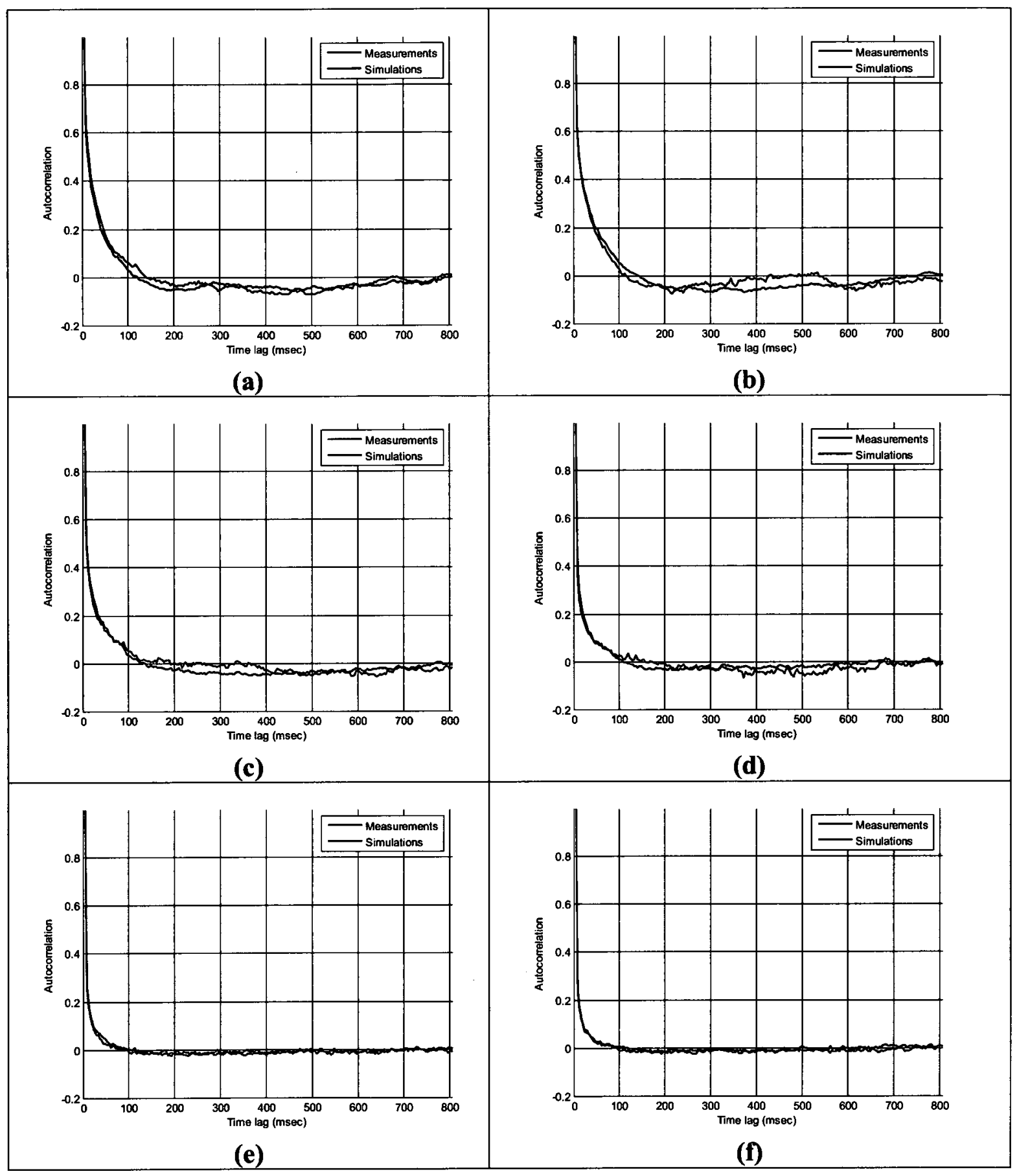

Figure 4.9. Comparison of the autocorrelation of time series derived from measurements and those derived from modelling for: (a) the rms DS of Group A. (b) the rms AS of Group A, (c) the rms DS of Group B, (d) the rms AS of Group B, (e) the rms DS of Group C, and (f) the rms AS of Group C. 
TABLE 4.5. Comparison of the Means of the CIs Identified in Simulated Time Series Data and in the Measured Data.

\begin{tabular}{|c|c|c|c|c|}
\hline Threshold & Measurements & \multirow{2}{*}{$\begin{array}{c}\text { Simulation } \\
\text { (snapshots) }\end{array}$} & (snapshots) & \multicolumn{2}{|c|}{ Error } \\
\cline { 4 - 5 } & & In snapshots & In msec \\
\hline 0.30 & 8 & 10 & 2 & 8 \\
\hline 0.40 & 15 & 17 & 2 & 8 \\
\hline 0.50 & 25 & 23 & 2 & 8 \\
\hline 0.60 & 34 & 29 & 5 & 20 \\
\hline 0.70 & 43 & 35 & 8 & 32 \\
\hline 0.80 & 44 & 39 & 5 & 20 \\
\hline 0.90 & 47 & 43 & 4 & 16 \\
\hline
\end{tabular}




\subsection{Summary}

This chapter focused on a study of the clustering nature of wireless channels as an effective tool in the modelling of the time-varying behaviour of such channels based on measured data through:

- Proposing an algorithm to identify SSMCs and track their parameters throughout their active intervals. This algorithm was used to find the statistics of the number of SSMCs, as well as the power, the rms DS, the rms AS, the length of the active intervals of each SSMC, and the length of the dormant intervals. It was found that the number of SSMCs can be modelled using Gamma distribution, and the GEV distribution can be used successfully to model the power of the different groups of SSMCs.

- Proposing a method to identify the points of changes among snapshots and to find the statistics of the lengths of the CIs.

- Introducing a new algorithm for modelling the change in power of the different groups of SSMCs where the increment/decrement step size was modelled using an exponential distribution. It was demonstrated that the generated power values for different groups of SSMCs have the same statistical time-varying behaviour as that of the groups of SSMCs identified in measured propagation data.

- An AR model was used to model the time-varying behaviour of the delay and angular parameters of the different groups of SSMCs.

The results from this chapter can be incorporated with existing channel models to characterize the behaviour of the multipath clusters within the simulated drops. For example, the length of the drops, and the change in the power, rms DS, and rms AS of simulated multipath clusters can be controlled using the measurement-based models reported herein, whereas heretofore, such intervals and parameter values were chosen more or less arbitrarily. 


\section{CHAPTER 5:}

\section{UTILIZING CLUSTERS FOR DYNAMIC SPATIAL OPPORTUNISTIC MULTIPLE ACCESS}

\subsection{The Clustering Nature of Wireless Channels}

As already discussed in Chapter 4, in multipath environments, radio signals propagate from the $\mathrm{Tx}$ to the $\mathrm{Rx}$ over multiple different propagation paths, resulting in the reception of multiple MPCs. Each MPC can be characterized by its power, phase, AOD, AOA and delay. Propagation measurement results have shown that the MPCs are not received uniformly in spatio-temporal space. Instead, energy is concentrated in clusters associated with reflections, scattering, and diffraction by interacting objects $[35$, 36]. Clusters have been defined in the literature as accumulations of MPCs that have similar spatio-temporal parameters (e.g., AOD, AOA and delay) and share the same longterm evolution, i.e., they stay intact over time $[35,37,38]$. Visual inspection was used to identify clusters in early research that analyzed outdoor propagation measurements, and the existence of between 1 and 14 clusters on the type of channel under consideration here has been previously reported $[35,38,39]$.

Because of the movement of either the $\mathrm{Tx}$, the $\mathrm{Rx}$, or both, as well as the movement of objects in the environment of operation that interact with propagating radio waves, the time-varying nature of wireless channels increases the multiuser diversity. The multiuser diversity can be defined as the likelihood at any given time to have a number of users that receive stronger signals than other users via well-positioned IOs that give rise to multipath clusters with high powers. The existence of clusters therefore makes it possible, at alternate times, to serve the users that receive signals with the greatest SNR on a priority basis through the implementation of different dynamic spatial-opportunistic multiple-access techniques. This maximizes overall system throughput. 
In this chapter, different techniques that utilize the existence of clusters to improve communication system throughput are introduced. Sections 5.2 and 5.3 consider utilizing multipath clusters on multiple antenna broadcasting channels (i.e., in systems where multiple antennas at the BS transmit information to multiple Rxs). Section 5.4. considers utilizing multipath clusters in cognitive radio systems.

\subsection{Angle-of-Departure-Aided Opportunistic Space-Division Multiple Access in MIMO Systems}

\subsubsection{Limited Feedback Techniques in Multiuser MIMO Broadcast Systems}

The use of MIMO systems is among the most promising methods to achieve high throughputs over wireless broadcast channels, when the BS is equipped with multiple antennas and each user can have more than one antenna. However, achieving the promised MIMO high throughputs requires that Channel State Information (CSI) be known at the Tx [59]. In practice, full CSI is only available at the $\mathrm{Rx}$, whereas the BS is fed back only an incomplete version of the available information because of noise, channel estimation error, delay and limited feedback bandwidth.

There are several techniques that increase the sum capacity, which is defined as the sum of rates at which users can jointly decode the receive data reliably, of a system by ensuring that the BS has information about the transmit channels to each user using a limited number of feedback bits. These techniques have been proposed in [60 - 64]. One effective technique that is used to reduce the amount of feedback and increase the system sum capacity is to take advantage of the opportunistic nature of a multiuser communication system. Opportunistic multiuser communications allows the BS to schedule the transmission in such a way that it targets the users that experience the best channel conditions [65 - 71]. If the channels are time-varying, most users get served, since different users receive the strongest signals at different (alternate) times. Examples of such techniques are

- opportunistic beamforming (OBF) $[66,67]$,

o opportunistic beamforming with beam selection (OBF-S) [68], 
○ opportunistic space-division multiple access (OSDMA) [69],

o opportunistic space-division multiple access with beam selection (OSDMA-S) [70], and

- opportunistic Grassmannian beamforming (OGB) [71].

When these techniques are employed, the BS transmits training sequence(s) (i.e., beams), collects feedback from all users, and then schedules the transmission to the user (or users) with the best channel conditions. Although all the opportunistic beamforming techniques are used to increase sum capacity by taking advantage of knowledge of the instantaneous channel conditions associated with communications to each user, they use different criteria to select the user(s) targeted for data transmission.

When OBF is used, the BS transmits a training sequence using a random beam (i.e., the phase and the amplitude of the signal fed to each $\mathrm{Tx}$ element changes in pseudorandom fashion); each user feeds back the SNR of signals it receives; then the user with the highest SNR is selected for service by the BS $[66,67]$. For systems with only a few users, the OBF-S technique, which is a modified version of OBF, is used. When the OBF-S technique is employed, more than one time slot is used to send multiple random beams (one beam at a time); each user feeds back the SNR associated with each transmitted beam; then the user with the highest SNR is selected for service by the BS [68]. The OSDMA technique has the objective of enabling the serving of more than one user simultaneously. This is accomplished when the BS transmits $N$ ( $N$ is the number of the transmit antennas) orthogonal beams, and each user reports the best beam and the corresponding signal-to-interference-plus-noise ratio (SINR). The BS then selects $N$ users to be served in such a way that the instantaneous sum capacity of the system is maximized [69]. With the OSDMA-S technique, $N$ orthogonal beams are transmitted in more than one time slot ( $N$ beams in each time slot). Each user reports the best beam and the associated SINR in each time slot. The BS selects $N$ users to be served simultaneously in such a way that the instantaneous sum capacity of the system is maximized [70]. The OGB technique has the objective of serving $N+1$ users simultaneously by constructing 
$N+1$ beams using the Grassmannian package technique and using a selection criteria identical to the one used with OSDMA [71].

This section introduces a novel technique that uses knowledge of AODs at the BS of the strongest signal received by each user to design multiple opportunistic beams so that advantage is made of the clustering nature of wireless channels.

Section 5.2.2. presents the system model. Section 5.2.3. provides an explanation of AOD estimation, where each user estimates the AOD at the BS of the strongest beam it receives. The proposed technique, herein referred to as the angle-of-departure opportunistic space-division multiple access (AOD-OSDMA) technique, is introduced in Section 5.2.4. Comparisons between the throughputs achievable with the new AODOSDMA and the well-known OSDMA-S technique are presented in Section 5.2.5.

\subsubsection{System Model and Frame Structure}

Consider a broadcasting system with $U$ mobile users and a BS equipped with an $N$-element UCA. At the BS two settings are assumed for the antenna configuration: a UCA with 4 elements and a UCA with 8 elements (i.e., $N=4$ or $N=8$ ). Each user's terminal is equipped with $M$ antennas $(M=1,2,3$, or 4$)$. The antenna configuration at each user's terminal is assumed to be a UCA with an inter-element spacing equal to half a wavelength at the operating frequency (i.e., for $M>1$, the angular difference between each adjacent element in the UCA array is equal to $2 \pi / M$ and the array radius is adjusted accordingly so that the inter-element spacing is fixed at half a wavelength at the operating frequency). For $M>2$, such antennas might be implemented, for example, on the backside of LCDs on laptop terminals.

Given the conditions described above, a MIMO channel $H_{u}^{M \times N}$ between a BS and each user $(u)$ is considered, and is assumed to remain constant within the duration of a single frame. The user channel realizations $H_{1}^{M \times N}, H_{2}^{M \times N}, \ldots . ., H_{U}^{M \times N}$ change between consecutive frames according to a cluster-based channel model described in Section 5.2.5. 
In this work, all AODs and AOAs are assumed to be in the horizontal plane. This assumption is supported by published work that reports that received signal energy is concentrated around the azimuth plane [72]. It is also assumed that the system bandwidth is small so that multipath signals cannot be resolved in the delay domain.

For the frame structure, it is assumed that every time slot of length $T$ has a training period that consists of $K$ minislots each of length $\tau$, and the remaining $T-K \tau$ duration is used for data transmission. The length of the training period is different for the different techniques under study. For example, the proposed AODOSDMA technique uses $K=1$, while OSDMA-S optimizes the value of $K$ in such a way that maximizes the sum capacity where $K_{\text {optimum }} \geq 1$ [70].

\subsubsection{Angle-Of-Departure Estimation and Joint Tx-Rx Beamforming}

This section explains how each user can: a) estimate the AOD at the BS of the strongest received beam, and $b$ ) use the calculated AOD to perform joint $T x-R x$ beamforming towards the IO that results in the strongest cluster.

\subsubsection{Angle-of-Departure Estimation}

If each Tx antenna at the BS transmits a pilot symbol $s_{n}$ (where $s_{1}, s_{2}, \ldots ., s_{N}$ is a set of mutually orthogonal signals), then, under the system setup explained in Section 5.2.2., the signal received by the $u$-th user is given by

$$
y_{u}^{M \times 1}=H_{u}^{M \times N}\left[\begin{array}{c}
s_{1} \\
s_{2} \\
\vdots \\
s_{N}
\end{array}\right]+\Xi_{u}^{M \times 1}=\left[\begin{array}{cccc}
h_{1,1, u} & h_{1,2, u} & \cdots & h_{1, N, u} \\
h_{2,1, u} & h_{2,2, u} & \cdots & h_{2, N, u} \\
\vdots & \vdots & \ddots & \vdots \\
h_{M, 1, u} & h_{M, 2, u} & \cdots & h_{M, N, u}
\end{array}\right]\left[\begin{array}{c}
s_{1} \\
s_{2} \\
\vdots \\
s_{N}
\end{array}\right]+\Xi_{u}^{M \times 1}
$$

where $H_{u}^{M \times N}$ is the matrix representing the radio channel between the BS and the $u-t h$ user, $h_{m, n, u}$ is the matrix coefficient representing the channel between the $n$-th Tx antenna at the BS and the $m$-th antenna at the $u$-th user station, $s_{n}$ is the pilot signal transmitted 
from the $n$-th Tx antenna at the BS and $\Xi_{u}^{M \times 1} \in C^{M \times 1}$ is complex zero-mean additive white Gaussian noise.

The relationship between channel coefficients, the AODs at the BS and the AOAs at the $u$ th user station of associated MPCs can then be written as [73, 48]:

$$
H_{u}^{M \times N}=\sum_{l=1}^{L_{u}}\left\{\frac{\alpha_{l, u} e^{j \Phi_{l, u}}}{\sqrt{M N}}\left[\begin{array}{c}
e^{j k a_{u} \cos \left(\varphi_{r x, l, u}-\phi_{1, u}\right)} \\
e^{j k a_{u} \cos \left(\varphi_{x, l, u}-\phi_{2, u}\right)} \\
\vdots \\
e^{j k a_{u} \cos \left(\varphi_{r, l, l u}-\phi_{M, u}\right)}
\end{array}\right]\left[\begin{array}{c}
e^{j k a_{B S} \cos \left(\varphi_{x x, l, u}-\phi_{1, B S}\right)} \\
e^{j k a_{B S} \cos \left(\varphi_{x x, l, u}-\phi_{2, B S}\right)} \\
\vdots \\
e^{j k a_{B S} \cos \left(\varphi_{t x, l, u}-\phi_{N, B S}\right)}
\end{array}\right]^{T}\right\}
$$

where $\phi_{n, B S}$ is the angular position of the $n$-th element in the BS array and is given by $\phi_{n, B S}=2 \pi n / N, n=1, \ldots ., N ; \phi_{m, u}$ is the angular position of the $m$-th element in the $u$-th user array and is given by $\phi_{m, u}=2 \pi m / M, m=1, \ldots ., M ; a_{B S}$ and $a_{u}$ are the radii of the UCAs at the BS and the $u$-th user station, respectively; $k=2 \pi / \lambda$ is the wavenumber at the operating frequency and $\lambda$ is the corresponding wavelength; $L_{u}$ is the total number of MPCs associated with the channel between the BS and the $u$-th user; and $\alpha_{l, u}, \Phi_{l, u}, \varphi_{t x, l, u}$ and $\varphi_{r x, l, u}$ are the complex amplitude, phase, azimuth AOD, and azimuth AOA of the l-th such MPC.

Assume that the $N$ pilot signals transmitted by the BS are known by all users, so that each user can get an estimate of the channel matrix $\hat{H}_{u}^{M \times N}$ from the received signal $y_{u}^{M \times 1}$. Given the limited number of antenna elements at the BS and at the users' terminals, only a limited number of beams $\left(B_{u} \ll L_{u}\right)$ can be detected from the estimated channel, where each beam consists of several MPCs with close AODs and AOAs. Thus, (5.2) can really only be estimated as: 


$$
\hat{H}_{u}^{M \times N}=\sum_{b=1}^{B_{u}}\left\{\frac{\alpha_{b, u} e^{j \Phi_{b, u}}}{\sqrt{M N}}\left[\begin{array}{c}
e^{j k a_{u} \cos \left(\varphi_{x, b, u}-\phi_{1, u}\right)} \\
e^{j k a_{u} \cos \left(\varphi_{r, b, u}-\phi_{2, u}\right)} \\
\vdots \\
e^{j k a_{u} \cos \left(\varphi_{x, b, u}-\phi_{M, u}\right)}
\end{array}\right]\left[\begin{array}{c}
e^{j k a_{B S} \cos \left(\varphi_{x, b, u}-\phi_{1, B S}\right)} \\
e^{j k a_{B S} \cos \left(\varphi_{x, b, u}-\phi_{2, B S}\right)} \\
\vdots \\
e^{j k a_{B S} \cos \left(\varphi_{x, b, u}-\phi_{N, B S}\right)}
\end{array}\right]^{T}\right\}
$$

where $B_{u}$ is the total number of resolvable beams linking the BS and the $u$-th user, and $\alpha_{b, u}, \Phi_{b, u}, \varphi_{t x, b, u}$ and $\varphi_{r x, b, u}$ are the complex amplitude, phase, azimuth AOD, and azimuth AOA of the $b$-th resolvable beam.

The information needed to estimate the AOD of the main beams is embedded in the coefficients of the estimated channel. The AOD of the strongest beam to the $\boldsymbol{u}$-th user $\left(\varphi_{t x, s t, u}\right)$ can be found by a simple beamscan [48]:

$$
\varphi_{t x, s t, u}=\arg \max _{0<=\varphi_{t x}<2 \pi} \sum_{m=1}^{M}\left|\hat{h}_{m, u} S V_{\varphi_{t r}, B S}^{H}\right|,
$$

where $|. .$.$| denotes absolute value, (. .)^{H}$ is the Hermitian transpose, $\underline{\hat{h}}_{m, u}$ is the $m$-th row of $\hat{H}_{u}^{M \times N}$ (i.e., the estimated coefficients of the channel between the Tx elements at the BS and the $m$-th antenna element at the $u$-th user station), and $S V_{\varphi_{t}, B S}$ is the BS elementspace UCA steering vector in the $\varphi_{t x}$ direction, which is defined as [73]

$$
S V_{\varphi_{x}, B S}=(1 / \sqrt{N})\left[e^{j k a_{B S} \cos \left(\varphi_{t x}-\phi_{1, B S}\right)}, e^{j k a_{B S} \cos \left(\varphi_{x x}-\phi_{2, B S}\right)}, \ldots \ldots, e^{j k a_{B S} \cos \left(\varphi_{x x}-\phi_{N, B S}\right)}\right]
$$

Note that to reduce the computational complexity associated with estimating $\varphi_{t x, s t, u}$, the search in (5.4) can be done by limiting the processing to only a single element of the antenna array at the $u$-th user station.

\subsubsection{Joint Tx-Rx Beamforming}

Each user estimates the AOA (if $M>1$ ) of the strongest beam, in addition to its $\mathrm{AOD}$, and performs a joint $\mathrm{Tx}-\mathrm{Rx}$ beamforming toward the IO producing the strongest cluster at both the BS and the user terminals simultaneously. 
The AOA of the strongest beam received by the $u$-th user can be found as:

$$
\varphi_{r x, s t, u}=\arg \max _{0<=\varphi_{r x}<2 \pi}\left|S V_{\varphi_{r x}, u}^{*} \hat{H}_{u}^{M \times N} S V_{\varphi_{x, s, u}}^{H}, B S\right|,
$$

where $(.)^{*}$ denotes the complex conjugate, $S V_{\varphi_{t, x, s, u}, B S}$ is the BS element-space UCA steering vector of the strongest beam associated with the $u$-th user, and $S V_{\varphi_{r}, u}$ is the element-space UCA steering vector at the $u$-th user station in the $\varphi_{r x}$ direction, which is defined as [73].

$$
S V_{\varphi_{x}, u}=(1 / \sqrt{M})\left[e^{j k k_{u} \cos \left(\varphi_{x}-\phi_{, u}\right)}, e^{j k a_{u} \cos \left(\varphi_{x}-\phi_{, u}\right)}, \ldots \ldots, e^{j k a_{u} \cos \left(\varphi_{x}-\phi_{M, u}\right)}\right]
$$

The SNR associated with the strongest beam received by the $u$-th user, which results from the joint $\mathrm{Tx}-\mathrm{Rx}$ beamforming, is a scaled version of the overall channel gain and can be written as:

$$
\operatorname{SNR}\left(\varphi_{t x, s t, u}, \varphi_{r x, s t, u}\right)=\frac{\left|S V_{\varphi_{x, s, t, u}, u}^{*} \hat{H}_{u}^{M \times N} S V_{\varphi_{x, s, t, u}, B S}^{H}\right|^{2}}{1 / \rho},
$$

where $\rho$ is the input SNR (i.e., the time average SNR at the input of each single branch) at each user's terminal.

The computational complexity of estimating the AOD at the BS of the strongest beam received by the $u$-th user is a function of the number of antenna elements at the BS and the step size of the beamscan search represented by (5.4). For example, if each user transmits the AOD information using $\boldsymbol{x}$ feedback bits, as presented in Section 5.2.5., then a step size of $2 \pi / 2^{x}$ is used in (5.4) and consequently $2^{x} . N$ complex multiplications, and almost the same number of complex additions, are required at each user terminal. Similarly, the computational complexity of estimating the AOA of the strongest beam received by the $u$-th user is a function of the number of antenna elements at the user 
station and the step size of the beamscan search represented by (5.6). However, the step size of the search in (5.6) is not restricted by the feedback bits constraint. Instead, it depends solely on the computational power of the user terminal.

\subsubsection{The Angle-Of-Departure Opportunistic Space-Division Multiple Access (AOD- OSDMA) Technique}

The objective of using the novel AOD-OSDMA technique described herein is to make the Tx direct most of the transmitted energy towards the best available propagation paths (i.e., the strongest clusters) to $N$ users simultaneously, in such a way that the instantaneous system throughput is maximized. This objective can be achieved through the following steps:

Step 1-During the training minislot two tasks are performed:

a. Each Tx antenna transmits a pilot symbol $s_{n}$, where $s_{1}, s_{2}, \ldots, s_{N}$ make up a set of mutually orthogonal signals.

b. Each user $u$ processes the received pilot signals, estimates the $\varphi_{t x, s t, u}$ and the $\varphi_{r x, s t, u}$, and calculates the associated SNR (i.e., $\left.\operatorname{SNR}\left(\varphi_{t x, s t, u}, \varphi_{r x, s t, u}\right)\right)$, as described in Section 5.2.3. Then the estimated $\varphi_{t x, s t, u}$ and $\operatorname{SNR}\left(\varphi_{t x, s t, u}, \varphi_{r x, s t, u}\right)$ are fed back to the BS.

Step 2-After collecting the feedback information, the AOD-OSDMA technique involves conventional beamforming at the BS to form beams toward the IOs best positioned to deliver the greatest SNRs to $N$ different users. Even though all such beams have the same power (5.5), they result in a different SNR at each targeted user, which is a function of the characteristics of the IOs that produce the strongest cluster (5.8). The AOD-OSDMA technique then requires the selection of $N$ users such that the sum of SNRs at the user stations is maximized, while the sum of correlations among the $N$ beamforming vectors at the BS is minimized. This selection criterion can be met using algorithm 5.1, described below. 
The main steps in this algorithm are as follows:

a. Select the user with the highest received SNR and add it to the list of the users to be served (Steps 2 and 3).

b. Artificially scale down (i.e., weight) the SNR of the signal received by each of the remaining users to reflect the effect of the correlation between the beam steering vector associated with beamforming to the selected user and that associated with beamforming to the subject user. The objective behind this step is to improve the performance of the system by avoiding the design of transmission beams with close AODs. This objective is met by penalizing the users whose beamforming vectors are correlated with that of the selected users (i.e., the users whose strongest clusters are close in the AOD domain to the selected cluster). The SNR of the $u$-th users is artificially scaled down through its multiplication by $\left\{1-\left|r_{u}\right|\right\}$ where $r_{u}$ is the correlation between the beamforming vector of the selected user and that of the $u$-th user (Step 5).

c. Delete the information associated with communications to the selected user from the available lists (Steps 7 and 8).

d. Search for the next user to be served (Step 9). 


\section{Algorithm 5.1: Steps for selecting $N$ users to be served using the AOD-OSDMA}

Inputs

$U$ is the total number of users

$N$ is the number of antenna elements at the BS

UsersID $=[1,2,3, \ldots, U]$ is the list of the IDs of the available users

$S N R=\left[S N R_{1}, S N R_{2}, \ldots . ., S N R_{U}\right]$ is the list of the reported received SNRs

$A O D=\left[A O D_{1}, A O D_{2}, \ldots ., A O D_{U}\right]$ is the list of the reported AODs

Initializations

Selected $=[]$ is the list of the users to be selected for transmission Available $=U$ is the number of the available users

No-Of-Served-Users $=\min ([N U])$

\section{$\underline{\text { Start search }}$}

1- For $i=1$ : No-Of-Served-Users

2- Find the position ( $p$ ), within the UsersID list, of the user with the maximum SNR

3- Selected $(i)=$ UsersID $(p)$

4- For j=1: Available

5- $S N R(j)=S N R(j) \times\left\{1-\left|S V_{A O D(j), B S} S V_{A O D(p), B S}^{H}\right|\right\}$

6- Next $j$

7- Delete $S N R(p)$ from the SNR list, delete $A O D(p)$ from the AOD list, and delete UsersID(p) from the UsersID list

8- Available $=$ Available -1

9- Next $i$

10- Return (Selected)

The throughput resulting from using the AOD-OSDMA technique can be evaluated as follows. Let $S V_{\varphi_{t, s, s, \text { Selected }(n)}, B S}$ and $S V_{\varphi_{n, s, t, \text { Seleced }(n)}, \text { Selected }(n)}, n=1,2, . ., N$ (assuming $U \geq N$ ), be the $\mathrm{Tx}$ (at the $\mathrm{BS}$ ) and the $\mathrm{Rx}$ (at the user station) steering vectors associated with the $N$ users that are selected by algorithm 5.1. Then the achieved sum capacity is calculated as:

$$
R=(T-\tau)\left\{\sum_{n=1}^{N} \log _{2}\left(1+\operatorname{SINR}_{n}\right)\right\}
$$


where $S I N R_{n}$ is the total SINR of the $n-t h$ user, and is calculated as:

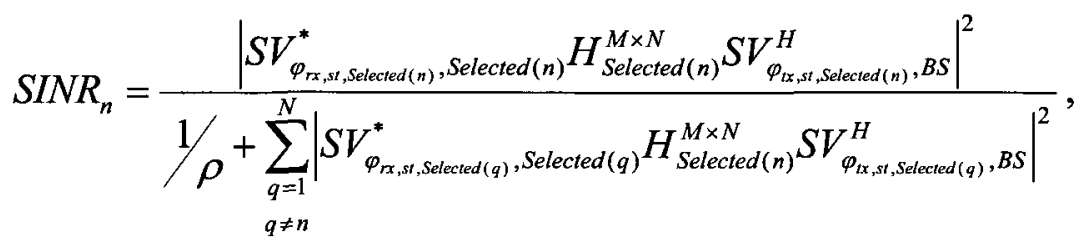

\subsubsection{Results and Discussion}

In this section, the sum capacity achieved using the proposed AOD-OSDMA technique is compared with that achieved using the OSDMA-S technique by MonteCarlo simulation for an urban micro-cell multi-antenna scenario. The WINNER channel model, a cluster-based model, is used to generate LOS and NLOS channel realizations where the number of generated clusters is 8 and 16, respectively, with 10 MPCs in each cluster [23]. Each MPC is characterized by its complex amplitude, delay, AOD and AOA. By adopting the array configurations at the BS and at the users' terminals, as described in section 5.2.2., and by neglecting shadowing, the channel matrix associated with the $u$-th user can be constructed as the sum of the MPCs as described in (5.2). In order to guarantee that any change in the $M \times N$ MIMO sum capacity is due to a change in spatial correlation properties and not to power variations, the $M \times N$ channel matrices generated are normalized as:

$$
\left\|H_{u}^{M \times N}\right\|_{F}^{2}=N
$$

where $\|. . .\|_{F}$ denotes a matrix Frobenius norm.

During the Simulations:

1- Imperfect channel estimation was assumed where the estimation error was modelled as a complex Gaussian random variable with $\sigma_{\text {error }}^{2}$ equal to $3 \%$ of the power in the impulse response estimated for the channel, which is a reasonable error that results from using practical channel estimation algorithms [74]. 
2- The exact (i.e., error free) values of the SINR or the SNR were reported to the BS in all cases (i.e., given that the major difference between the two techniques under consideration comes from the criterion used for designing and selecting the transmission beams, only the number of feedback bits needed to report the directional information, including, the AOD and the ID of the selected beam are considered).

3- 1000 trials were simulated with the input SNR of signals received at each user's terminal equal to $10 \mathrm{~dB}$, and $\tau / T=0.02$.

\subsubsection{Comparison of System Performance when using the AOD-OSDMA, and} OSDMA-S Techniques

Figures 5.1.-5.5., 5.7., and 5.9. show a comparison of system performance when using the AOD-OSDMA, and OSDMA-S techniques, as a function of the number of users in both LOS and NLOS scenarios. It is assumed that:

1- The OSDMA-S technique optimizes the number of training minislots (i.e., $K$ ) as in [70].

2- The AOD-OSDMA technique uses the same number of feedback bits as the OSDMA-S technique (i.e., only one of $2^{x}$ possible values for AOD is fed back where $\boldsymbol{x}$ is the number of feedback bits per user).

Two cases are studied: the one in which the users are equipped with a single antenna, and the general one in which the users are equipped with multiple antennas.

\section{Case 1: Users with a single antenna}

In case 1, each user is equipped with a single antenna. For the AOD-OSDMA technique, the inter-element spacing $(d)$ at the BS is fixed to $\lambda / 2$ to avoid wasting energy and causing interference, whereas for the OSDMA-S technique, $d$ took one of the values $\lambda / 2, \lambda, 2 \lambda$ and $5 \lambda$. Figures 5.1. and 5.2. compare the performance of the two techniques with $N=4$ and $N=8$, respectively. 
From the results, it is clear that:

- When the BS is equipped with $(N=4)$ elements, increasing $d$ results in improving the performance of OSDMA-S, with the greatest improvement being realized when spacing is changed from $\lambda / 2$ to $\lambda$. When $d \geq \lambda$ and $U \geq 25$ (i.e., large number of users), OSDMA-S outperforms AOD-OSDMA, for the following reasons: (a) as $d$ increases, the correlation among the signals at the different $\mathrm{Tx}$ elements is reduced (i.e., the channel coefficients get closer to the i.i.d. assumption of OSDMA-S); (b) as $U$ increases, the multiuser diversity effect increases; which increases the efficiency of the random OSDMA-S beam design criterion. Furthermore, the small number of the BS elements $(N=4)$ reduces the beamforming directivity of the BS antenna, which results in reducing the efficiency of the AOD-OSDMA. On the other hand, with a small number of users $(U<20)$, AOD-OSDMA outperforms OSDMA-S due to the non-random nature of the beam selection of AOD-OSDMA compared to the purely random beamdesign criterion OSDMA-S. (See Figure 5.1.)

- As the number of BS elements increases (i.e. to $N=8$ ), the efficiency of OSDMA-S decreases. This reduction in efficiency is due to the increase in the dimensionality of the randomly generated beams, which reduces the chances of opportunistically generating beams that are able to maximize the throughput of the system. Increasing $d$ still increases the OSDMA-S throughput. However, this improvement is less significant than it is in the case where $N=4$. On the other hand, increasing $N$ results in a significant improvement to system performance when using AOD-OSDMA due to increasing the beamforming directivity of the BS antenna (i.e., better focusing of the transmit energy in the desired direction). (See Figure 5.2.) 
(a)

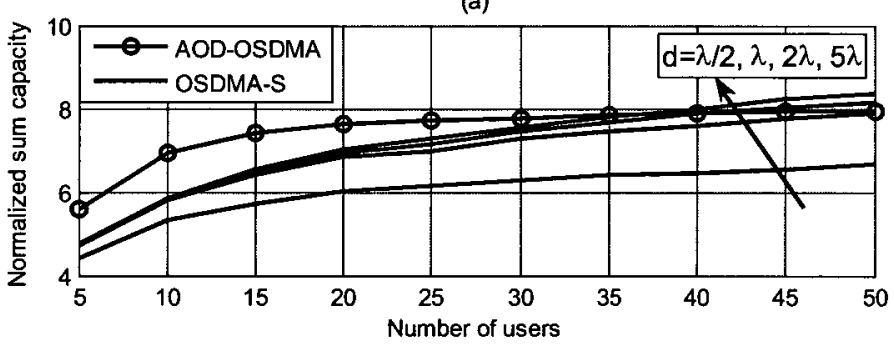

(b)

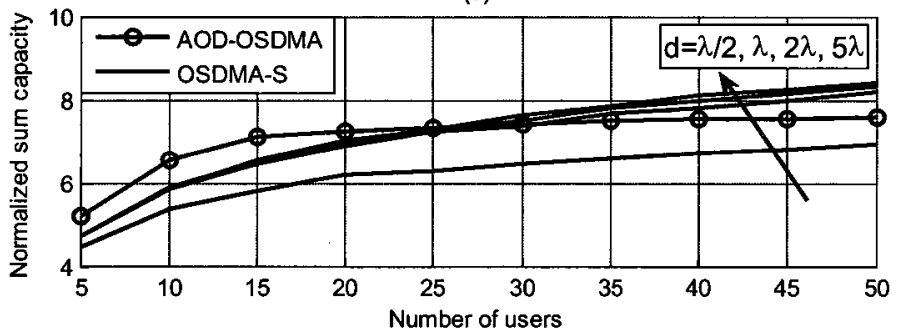

Figure 5.1: Comparison of system sum capacity when using AOD-OSDMA, and OSDMA-S with different inter-element spacings, as a function of the number of users: $S N R=10 \mathrm{~dB}, \tau / L=0.02, N=4, M=1$. (a) $L O S$ and (b) NLOS.

(a)

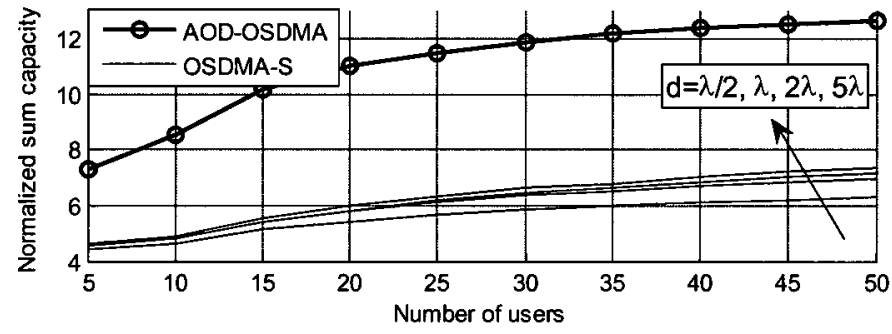

(b)

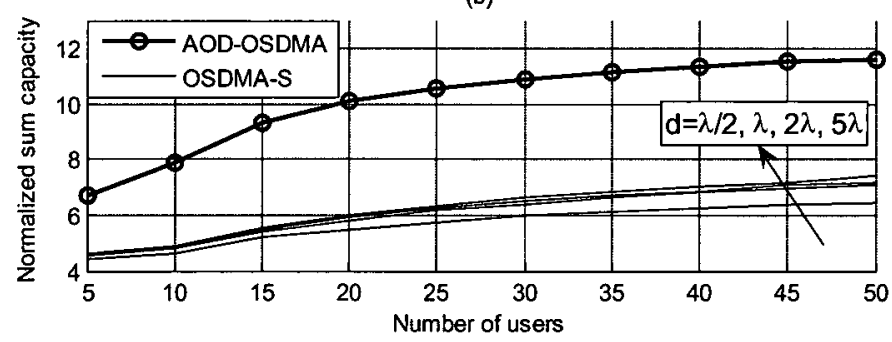

Figure 5.2: Comparison of system sum capacity when using AOD-OSDMA, and OSDMA-S with different inter-element spacings, as a function of the number of users: $S N R=10 \mathrm{~dB}, \tau / L=0.02, N=8, M=1$. (a) $L O S$ and (b) NLOS. 
(a)

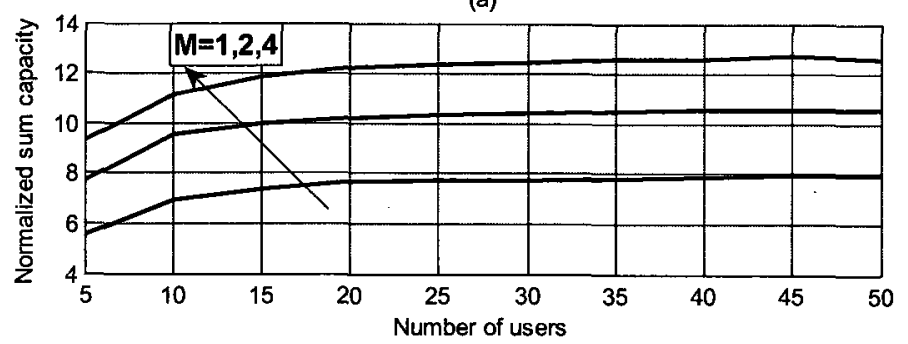

(b)

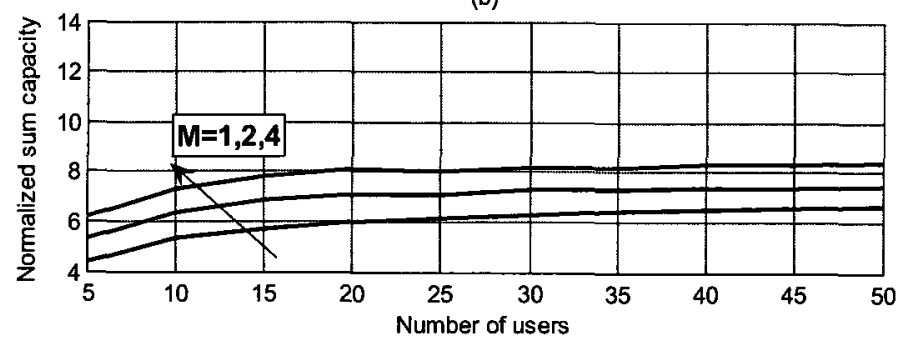

Figure 5.3: Effect of increasing the number elements at the user's terminals with SNR=10 dB, $\tau / L=0.02$, LOS , and N=4 (a) AOD-OSDMA and (b) OSDMA-S.

(a)

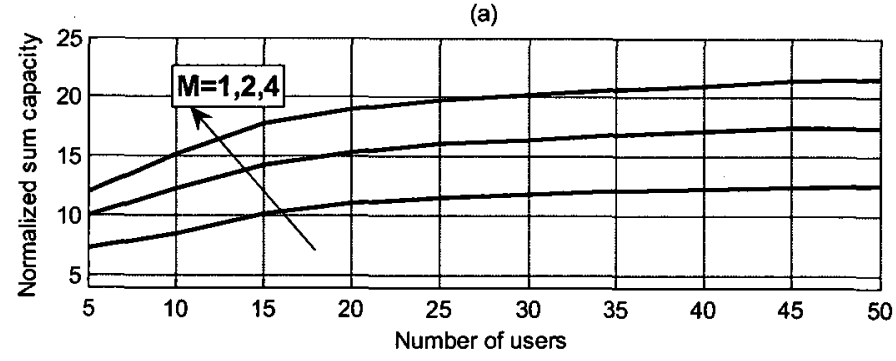

(b)

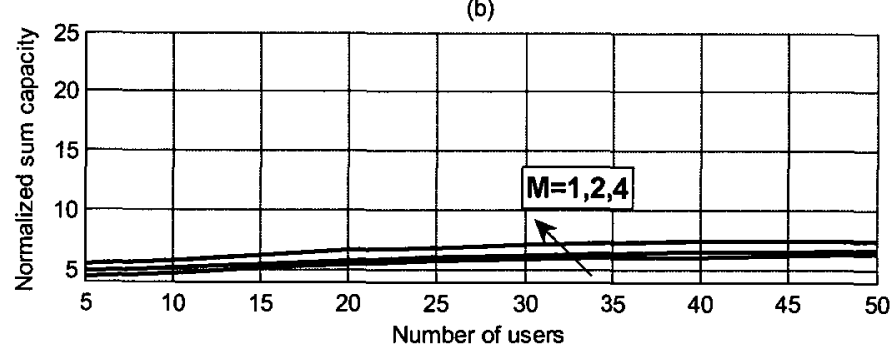

Figure 5.4: Effect of increasing the number elements at the user's terminals with SNR $=10 \mathrm{~dB}, \tau / L=0.02, \mathrm{LOS}$, and $\mathrm{N}=\mathbf{8}$ (a) AOD-OSDMA and (b) OSDMA-S. 


\section{Case 2: Users with multiple antennas}

In the case where each user is equipped with more than one antenna (results in figures 5.3., 5.4., 5.5., 5.7., and 5.9.), the following was found:

1- Compared to OSDMA-S, use of the AOD-OSDMA technique results in increasing the system capacity by up to $160 \%$ as depicted in TABLE 5.1 .

TABLE 5.1. Capacity Improvement of AOD-OSDMA Compared to OSDMA-S (with $d=\lambda / 2$ ) Capacities based on Simulations using the WINNER Model

\begin{tabular}{|l|c|c|c|c|c|c|}
\hline & \multicolumn{2}{|c|}{$\mathrm{U}=10$} & \multicolumn{2}{c|}{$\mathrm{U}=30$} & \multicolumn{2}{c|}{$\mathrm{U}=50$} \\
\cline { 2 - 7 } & LOS & NLOS & LOS & NLOS & LOS & NLOS \\
\hline $\mathrm{N}=4 ; \mathrm{M}=1$ & $30 \%$ & $22 \%$ & $23 \%$ & $15 \%$ & $19 \%$ & $9 \%$ \\
\hline $\mathrm{N}=4 ; \mathrm{M}=2$ & $49 \%$ & $48 \%$ & $43 \%$ & $42 \%$ & $42 \%$ & $39 \%$ \\
\hline $\mathrm{N}=8 ; \mathrm{M}=1$ & $85 \%$ & $70 \%$ & $102 \%$ & $81 \%$ & $99 \%$ & $79 \%$ \\
\hline $\mathrm{N}=8 ; \mathrm{M}=2$ & $142 \%$ & $122 \%$ & $163 \%$ & $142 \%$ & $163 \%$ & $138 \%$ \\
\hline
\end{tabular}

2- System capacity when using AOD-OSDMA improves significantly as a result of increasing the number of antenna elements at the user terminals. This performance improvement is a direct result of performing joint $\mathrm{Tx}-\mathrm{Rx}$ beamforming in (5.10.) which can be explained as follows: (a) for each selected user, the BS directs the energy towards the IO that generates the cluster associated with the best channel to a specific user (i.e., due to the BS beamforming, each of the targeted IOs is illuminated mainly by the signal intended for a specific user with minimum illumination by signals intended for other users), and (b) each user performs a beamforming towards the IO from which it receives the most energy. The effect of (a) and (b) is to increase the SINR for each one of the selected users and, consequently, it increases the throughput of the system significantly.

3- System capacity when using OSDMA-S does not improve as significantly when the number of antenna elements at the user terminals is increased. This behaviour can be explained as follows. The random beams transmitted when using the OSDMA-S technique distribute the transmitted signals evenly in space (i.e., in all directions), which results in: (a) illuminating the IOs that generate strong 
cluster(s) with a smaller proportion of the transmitted energy, and (b) achieving an almost fixed SINR for all users in all directions. Consequently the beamforming at each user's terminal (toward the strongest cluster) is not able to reduce the interference effect but still helps increase SNRs slightly. 
(a)

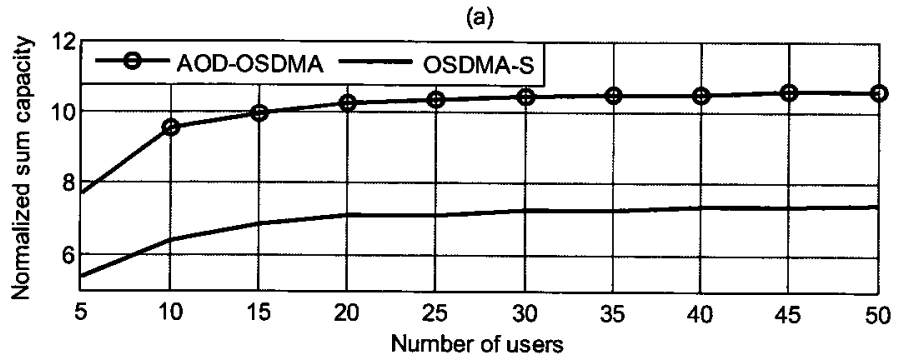

(b)

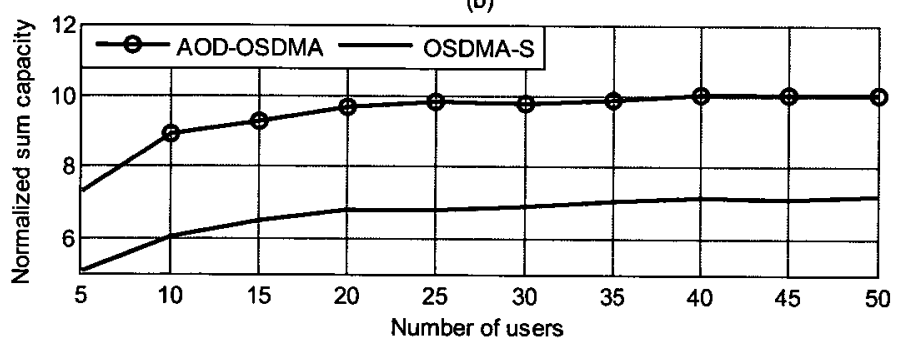

Figure 5.5: Comparison of system capacity when using AOD-OSDMA, and OSDMA-S $(d=\lambda / 2)$ as a function of the number of users: $S N R=10$ dB, $\tau / L=0.02, \mathbf{N}=4, M=2$. (a) LOS and (b) NLOS.

(a)

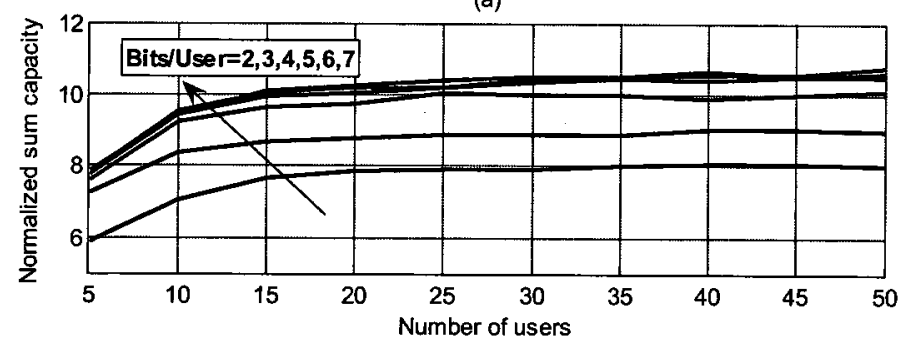

(b)

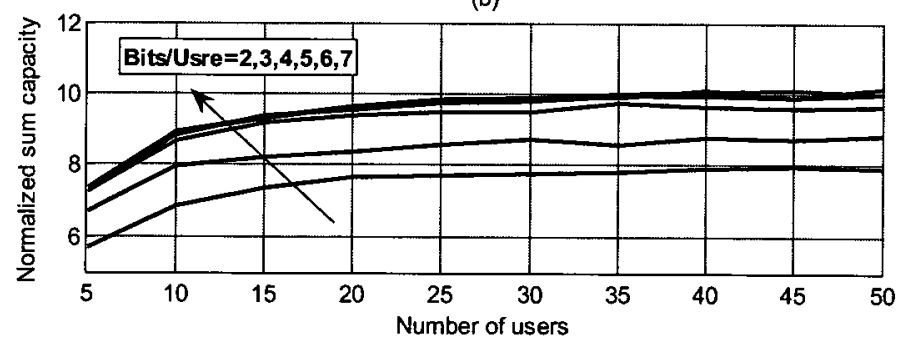

Figure 5.6: Effect of increasing the number of bits per user (used for AOD feedback) on system capacity when using AOD-OSDMA with $\mathrm{SNR}=10 \mathrm{~dB}, \tau / L=0.02, \mathbf{N}=4$, and $M=2$. (a) LOS and (b) NLOS. 
(a)

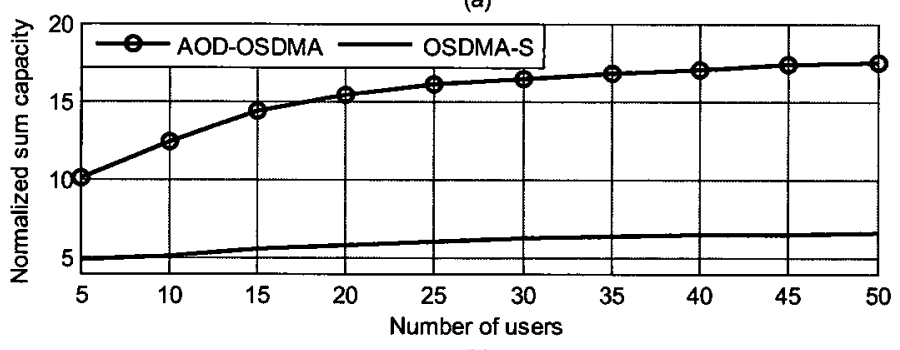

(b)

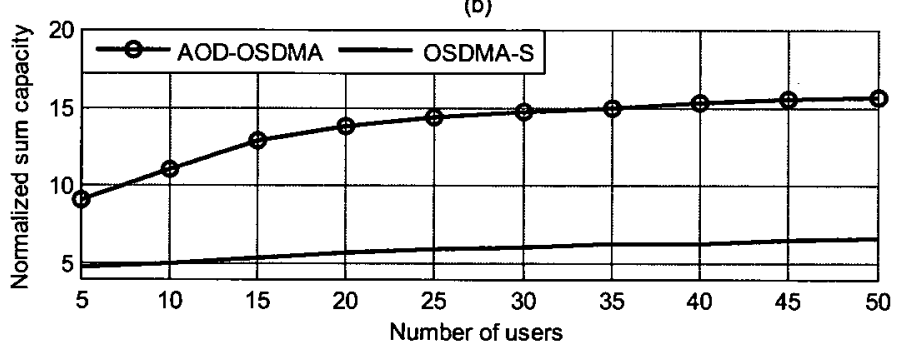

Figure 5.7: Comparison of system capacity when using AOD-OSDMA, and OSDMA-S $(d=\lambda / 2)$ as a function of the number of users: $S N R=10$ $\mathrm{dB}, \tau / T=0.02, \mathbf{N}=8, \mathrm{M}=2$. (a) LOS and (b) NLOS.

(a)

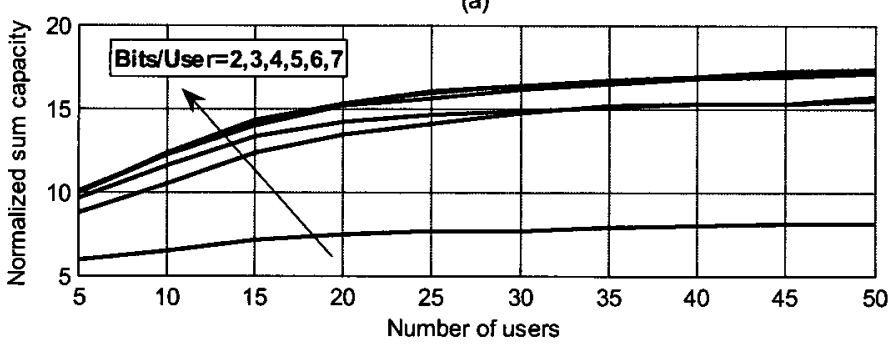

(b)

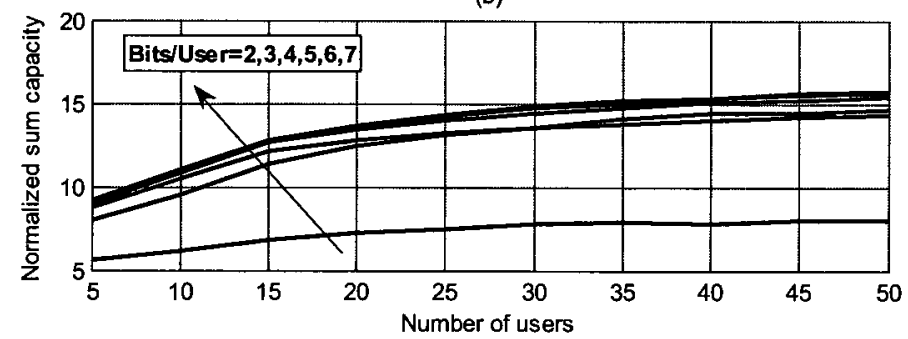

Figure 5.8: Effect of increasing the number of bits per user (used for AOD feedback) on system capacity when using AOD-OSDMA with SNR=10 dB, $\tau / T=0.02, N=8$, and $M=2$. (a) LOS and (b) NLOS. 
(a)

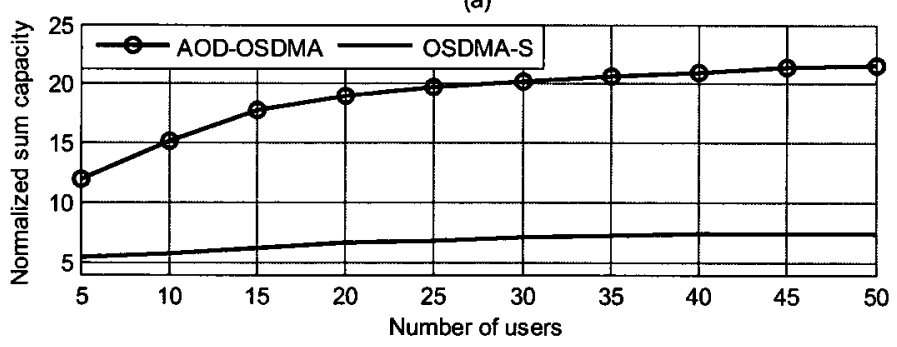

(b)

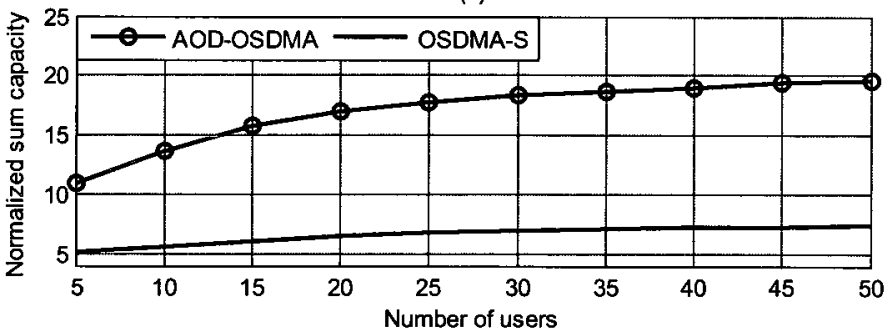

Figure 5.9: Comparison of system capacity when using AOD-OSDMA, and OSDMA-S $(d=\lambda / 2)$ as a function of the number of users: $S N R=10$ dB, $\tau / T=0.02, N=8, M=4$. (a) LOS and (b) NLOS.

(a)

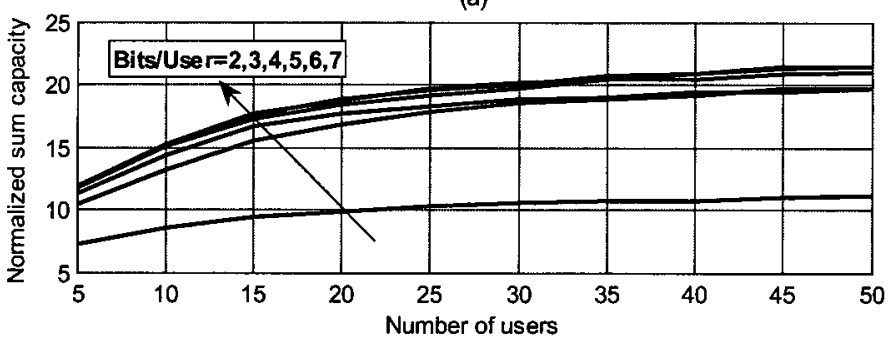

(b)

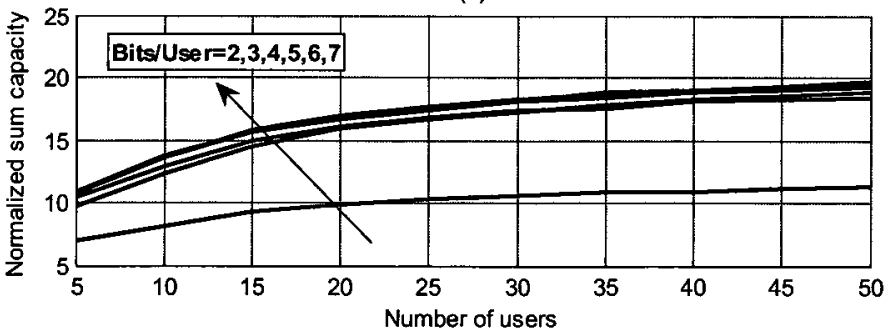

Figure 5.10: Effect of increasing the number of bits per user (used for AOD feedback) on system capacity when using AOD-OSDMA with $\mathrm{SNR}=10 \mathrm{~dB}, \tau / T=0.02, \mathrm{~N}=8$, and $M=4$. (a) LOS and (b) NLOS. 
5.2.5.2. Comparison of the Number of Feedback Bits per User used with the Two

Techniques

When using OSDMA-S, optimizing the number of training slots (i.e., $K$ ) using the method proposed in [70] results in the need for a minimum of 3 (a maximum of 5) training slots under the conditions of $\tau / T=0.02$ and having a high (low) number of users. This optimized number of training slots results in the need for the transmission of a minimum of 6 and 9 (a maximum of 10 and 15) feedback bits by each user in the case of $N=4$ and $N=8$, respectively. By comparing Figure 5.5. with Figure 5.6., Figure 5.7. with. Figure 5.8., and Figure 5.9. with Figure 5.10., it was found that, in all cases, the use of AOD-OSDMA with only 4 feedback bits per user resulted in significantly greater system sum capacity than that achievable when using the OSDMA-S; and, at the same time, AOD-OSDMA reduced this number by more than $33 \%$ and $55 \%$ for $N=4$, and $N=8$, respectively. 


\subsection{Modified Opportunistic Space-Division Multiple Access with Beam Selection}

\subsubsection{Motivation}

The AOD-OSDMA that was introduced in Section 5.2. outperforms the original OSDMA-S technique in terms of the system throughput. However, AOD-OSDMA requires each user to perform AOD estimation. The computational complexity associated with the AOD estimation might not be affordable in all cases. This section therefore introduces a simpler, but less efficient, transmission technique for use in multiple antenna broadcast systems that allows simultaneous communications with multiple users using a limited number of feedback bits. The proposed technique, referred to herein as Modified Opportunistic Space-Division Multiple Access with Beam Selection (Mod-OSDMA-S), is a modified version of the technique introduced in [70]. The new technique increases the throughput of the system by incorporating consideration of the clustering nature of wireless channels during the beam-design stage. Comparisons of capacities resulting from the use of the new technique against those achievable using OSDMA-S are made using the WINNER model. Results show that the proposed new technique outperforms the original technique by more than $10 \%, 20 \%$, and $25 \%$ when the number of users is low, medium, and high, respectively, while maintaining the same selection criterion, the same computational complexity, and using the same number of feedback bits.

Section 5.3.2. introduces the system model and the frame structure. The modified opportunistic space-division multiple access with beam selection (Mod-OSDMA-S) technique is introduced in Section 5.3.3. Finally, the results of a performance comparison between Mod-OSDMA-S, and OSDMA-S are presented in Section 5.3.4.

\subsubsection{System Model and Frame Structure}

A broadcast system in which $U$ users, each equipped with a single antenna, are served by a BS with an $N$-element UCA is considered. As a result, a multiple-input single-output (MISO) channel $h_{u}^{1 \times N}$ between the BS antennas and each user is considered and is assumed to remain constant within a frame duration. 
Regarding the frame structure, it is assumed that every time slot of length $L$ has a training period that consists of $K$ minislots each of length $\tau$, and the remaining $L-K \tau$ duration is used for data transmission. The number of training minislots $K$ is the same for both OSDMA-S and Mod-OSDMA-S, and this number is optimized as in [70].

\subsubsection{The Modified Opportunistic Space-Division Multiple Access with Beam Selection (Mod-OSDMA-S) Technique}

With the opportunistic space-division multiple-access with beam selection (OSDMA-S) technique, which was introduced in [70], the number of training minislots is optimized in such a way that the sum capacity is maximized. In each training minislot, $N$ orthogonal beams are transmitted, and each user reports the best beam and its corresponding SINR. After processing the reported information, the BS selects $N$ users to be served simultaneously so that the instantaneous sum capacity is maximized. OSDMA$\mathrm{S}$ designs the transmission random beams via singular value decomposition of randomly generated matrices of i.i.d. complex Gaussian coefficients.

By considering the clustering nature of wireless channels, it was found that the sum capacity that can be achieved using the OSDMA-S technique can be improved by changing the criterion for the design of the transmit beams, as detailed below:

\subsubsection{The Beam Design Criterion used in Mod-OSDMA-S}

In the use of Mod-OSDMA-S, the transmit beams would be designed using conventional beamforming, wherein the directions of the main lobes would be distributed uniformly over $2 \pi$ radians in azimuth.

The steering vector associated with the $n$-th beam transmit during the $k$-th training minislot in Mod-OSDMA-S can be written as $S V_{(\psi k+2 \pi(n-1) / N)}$, where $n=1,2, \ldots, N$; $\psi k$ is a random angle uniformly distributed between 0 and $2 \pi$; and $S V_{\varphi_{t x}}$ is the element-space UCA steering vector in the $\varphi_{t x}$ direction, which is defined as: 


$$
S V_{\varphi_{t x}}=(1 / \sqrt{N})\left[e^{j k a_{B S} \cos \left(\varphi_{x x}-\phi_{1}\right)}, e^{j k a \cos \left(\varphi_{t x}-\phi_{2}\right)}, \ldots \ldots, e^{j k a \cos \left(\varphi_{x x}-\phi_{N}\right)}\right]
$$

where $\phi_{n}$ is the angular position of the $n$-th element in the BS UCA and is given by: $\phi_{n}=2 \pi n / N, \quad n=1, \ldots ., N ; \quad a_{B S}$ is the radius of the BS array; $k=2 \pi / \lambda$ is the wavenumber at the operating frequency; and $\lambda$ is the corresponding wavelength.

\subsubsection{Selection of Users}

In both versions of OSDMA-S, the criterion used to select which users are to be served can be summarized as follows. For every training minislot $(k)$, each user feeds back the maximum SINR along with the index of the beam that maximizes it. The BS calculates the throughput $R_{k}$ at each minislot as:

$$
R_{k}=\sum_{n=1}^{N} \log _{2}\left(1+\max _{1 \leq u \leq U} S I N R_{u, n, k}\right),
$$

where $S I N R_{u, n, k}$ is the $S I N R$ at the $u$-th user, resulting from receiving the $n$-th beam in the $k$-th training minislot, and it is calculated as:

$$
S I N R_{u, n, k}=\frac{\left|h_{n} S V_{n, k}^{H}\right|^{2}}{1 / \rho+\sum_{\substack{j=1 \\ j \neq n}}^{N}\left|h_{n} S V_{j, k}^{H}\right|^{2}},
$$

where $\rho$ is the input SNR at each user station. The BS selects the set of $N$ different users (i.e., no user is assigned more than one beam) that maximizes $R_{k}$.

\subsubsection{Throughput Calculation}

Let $S V_{1}, S V_{2}, \ldots, S V_{N}$ be the steering vectors in the directions of the $N$ selected users that maximize (5.13). The achieved sum capacity can then be calculated as: 


$$
R=(L-\tau K)\left\{\sum_{n=1}^{N} \log _{2}\left(1+S I N R_{n}\right)\right\},
$$

where $\operatorname{SINR}_{n}$ is the total SINR of the $n$-th user.

\subsubsection{Results and Discussion}

In this section, the performance of the proposed Mod-OSDMA-S technique is compared with that of the OSDMA-S technique. A UCA with 8 elements is assumed as the BS antenna. It is assumed that each user is equipped with a single antenna, the SNR at each user terminal equal to $10 \mathrm{~dB}, \tau / L=0.02$, and the number of training minislots $(\mathrm{K})$ is optimized as in [70].

Channel realizations were generated using the WINNER Channel Model. The WINNER channel model, a cluster-based model, was used to generate the MPCs that comprise each channel realization. The urban micro-cell scenario was considered where the number of generated clusters equals 16, each cluster consists of $10 \mathrm{MPCs}$, and each MPC is described by its complex amplitude, AOA, and delay [23]. The channels between the user antenna and each of the BS antenna elements were constructed from knowledge of the parameters of their MPCs using plane-wave reconstructions [50].

Monte-Carlo simulations (1000 trials) were used to compare the performance of Mod-OSDMA-S and OSDAM-S as a function of the number of users. The inter-element spacing $(d)$ at the BS antenna array for the case of Mod-OSDMA-S was fixed at $d=\lambda / 2$; however, for OSDMA-S, $d$ took the values $\lambda / 2, \lambda, 2 \lambda$, and $5 \lambda$. From the results shown in Figure 5.11, it is clear that:

1. The performance of OSDMA-S improves as the inter-element spacings $(d)$ increases; however, even with $d=5 \lambda$, the performance of OSDMA-S is poorer than that of Mod-OSDMA-S. 
2. Mod-OSDMA-S improves the sum capacity by more than $10 \%, 20 \%$, and $25 \%$ when the number of users is 5,30, and 50, respectively, compared to the best throughput that can be achieved by OSDAM-S (i.e., with $d=5 \lambda$ ).

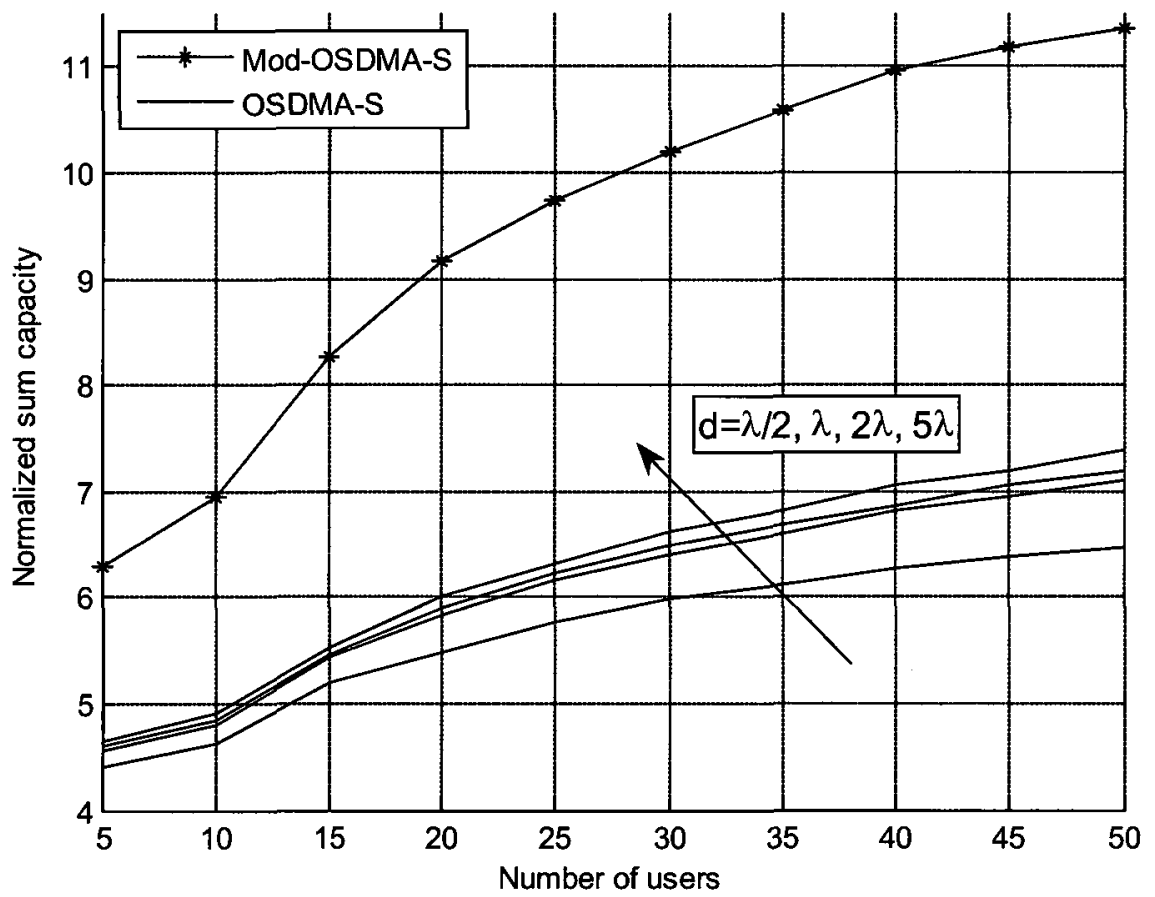

Figure 5.11: Performance comparison of Mod-OSDMA-S and OSDMA-S as a function of the number of users using the WINNER model: $S N R=10$ $\mathrm{dB}, N=8$, and $\tau / L=0.02$. 
Figure 5.12 illustrates the performance comparison of AOD-OSDMA, OSDMA$\mathrm{S}$, and Mod-OSDMA-S as a function of the number of users in LOS and NLOS propagation conditions, respectively. From Figure 5.12, we find that:

1- The AOD-OSDMA performs the best followed by the Mod-OSDMA-S while the OSDMA-S comes at last. Moreover, by increasing the number of the BS antennas from 4 to 8, the performance improvement of AOD-OSDMA and Mod-OSDMA-S over the OSDMA-S increases.

2- The relative improvement of the AOD-OSDMA over both the OSDMA-S and the mod-OSDMA-S decreases with increasing the number of users. This reduction in the relative improvement is due to the higher multiuser diversity which can be achieved by OSDMA-S and mod-OSDMA-S, where the probability for the randomly generated vectors to maximize the throughput increases as the number of users increases. 
(a)

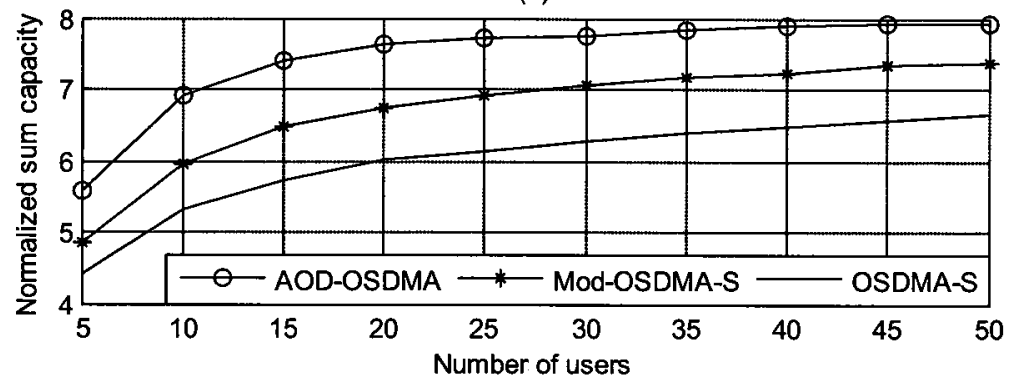

(b)

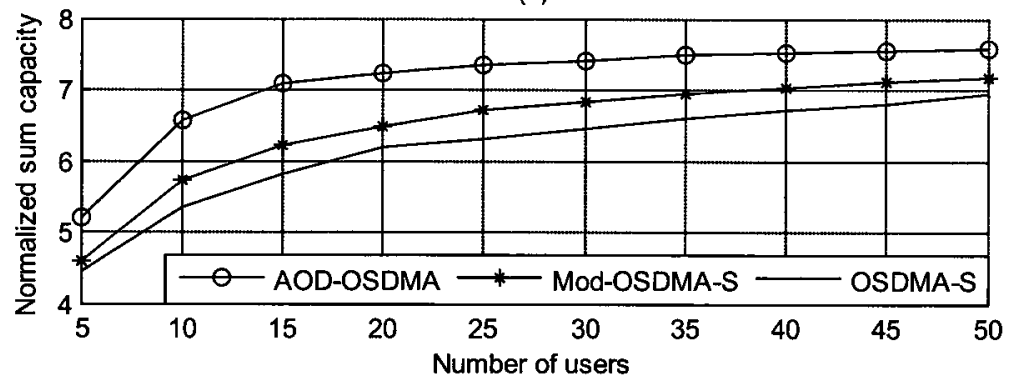

(c)

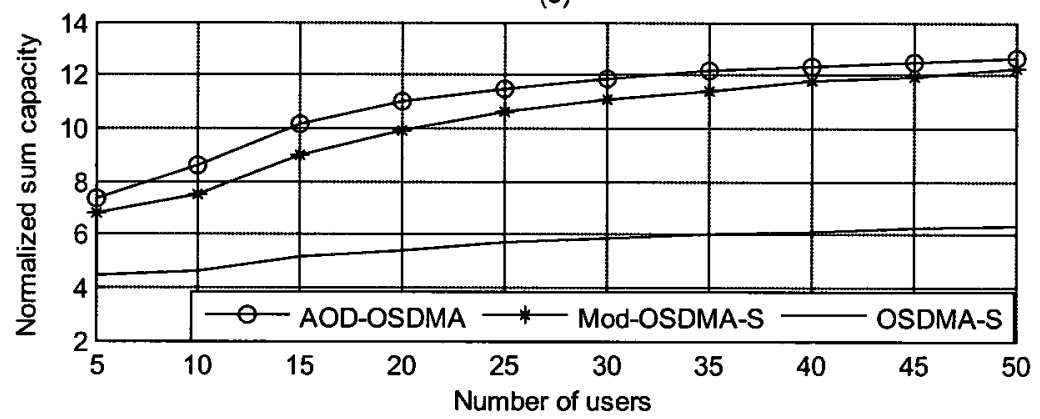

(d)

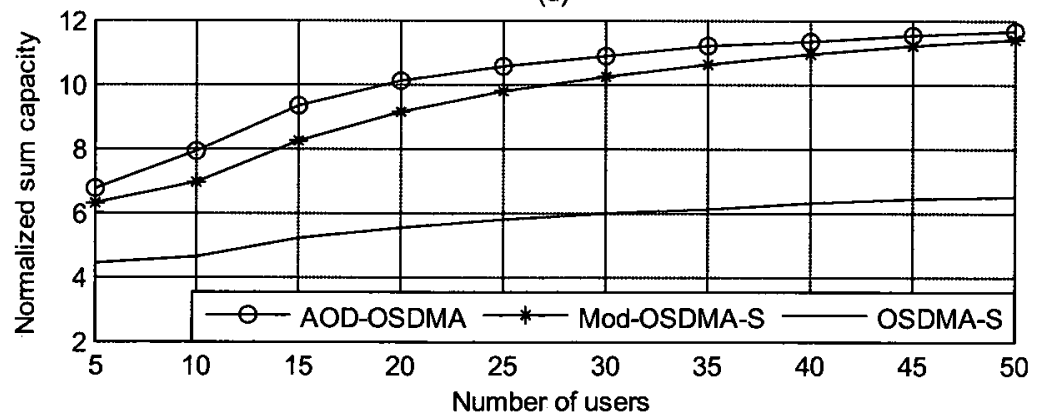

Figure 5.12: Performance comparison of AOD-OSDMA, OSDMA-S, and modOSDMA-S (with $d=\lambda / 2$ for all techniques) as a function of the number of users with $S N R=10 \mathrm{~dB}, \tau / L=0.02$, and $M=1$. (a) $L O S$, $\mathrm{N}=4$, (b) NLOS, N=4, (c) LOS, $N=8$, and (d) NLOS, $N=8$. 


\subsection{A Cluster-Based Spatial Opportunistic Spectrum Sharing Technique for Cognitive Radio Applications}

\subsubsection{Background: Cognitive Radio Technologies}

It is well known that today's wireless networks experience a rapid increase in spectrum demands for different wireless services, which results in scarcity in particular bands of the radio spectrum [75]. To meet future spectrum demands, cognitive radio was introduced as "a smart technology" that is capable of utilizing the spectrum efficiently by allowing multidimensional reuse of spectrum in space, frequency and time. Cognitive radio is defined as a wireless communication technology wherein the radio nodes are aware of their environments and can change their transmission and reception parameters in such a way that the overall efficiency of the system is maximized.

Cognitive radio improves spectrum utilization by introducing two concepts for spectrum sharing: overlaying spectrum sharing, and underlying spectrum sharing. With overlaying spectrum sharing, cognitive radio detects holes ${ }^{1}$ in the spectrum assigned to primary (higher priority) users (PUs), and assigns them to secondary (lower priority) users (SUs) [76 - 78]. On the other hand, the use of underlying spectrum sharing allows the simultaneous transmission by both SUs and PUs only if the interference caused by the SUs at the primary Rxs is kept below some acceptable threshold.

The main goal behind the notion of the underlying spectrum sharing is to support opportunistic spectrum-sharing strategies, which allow the coexistences of the PUs and SUs in frequency and time, as long as the required interference constraints are met [79$81]$.

The recent deployment of multiple antenna systems has encouraged the development of spectrum-sharing strategies that exploit the additional degrees of freedom provided by the spatial dimension (i.e., opportunistic beamforming). Several researchers have developed opportunistic beamforming approaches, assuming that the channels from the secondary Tx (SU-Tx) to both the secondary Rx (SU-Rx) and the primary Rx (PU-

\footnotetext{
${ }^{1}$ A spectrum hole is defined as " $a$ band of frequencies assigned to a primary user, but, at a particular time and specific geographic location, the band is not being utilized by that user".
} 
$\mathrm{Rx}$ ) are known perfectly at the SU-Tx [79, 82 - 85]. In most cases, this is a non-realistic assumption. On the other hand, less work has been done developing robust algorithms that can work with partial CSI $[80,86,87]$. In [86 and 80], it was assumed that the CSI between the SU-Tx and the SU-Rx is perfectly known at the SU-Tx, while partial CSI between the SU-Tx and PU-Rx is available at the SU-Tx.

This section proposes a spatial opportunistic algorithm that allows the coexistence of the PUs and SUs in both frequency and time, using practical assumptions such as: (a) limited feedback ( 8 bits/SU) from the SUs to the secondary base station (SBS) where the SBS uses these feedback bits to estimate the channel quality and the channel directionality of the SUs, (b) a minimum collaboration and feedback from the PU, where a maximum of 4 feedback bits are sent from the PU to the SBS to indicate whether the level of interference caused by the secondary transmissions is acceptable, and (c) a realistic channel model, where a cluster-based channel model (i.e., the WINNER model) is used to simulate/evaluate the proposed algorithm. In addition, the proposed algorithm does not have any requirement regarding the number of antenna elements at either the PU terminals or the primary base station (PBS).

\subsubsection{System Model and Interference Estimation}

The system under consideration is illustrated in Figure 5.13. It is assumed that there is one primary single-input single-output (SISO) link, between a PBS and a PU, which occupies all the frequency bands allocated for the type of service of interest, and transmits all the time, except for a short discovery window at the beginning of each frame. It is also assumed that a cognitive radio network is deployed in the same area and shares the same spectrum resources with the primary network. The secondary network consists of a SBS, equipped with an $N$-element antenna array serving $K$ cognitive SUs, each of which is equipped with an $M$-element antenna array. For both the SBS and the SUs the antenna configurations are assumed to be a UCA with an inter-element spacing equal to half a wavelength at the operating frequency (i.e., the angular difference between 
each adjacent elements in the UCA array is equal to $2 \pi / N$ at the SBS, and $2 \pi / M$ at the SU terminal, and the array radius is adjusted accordingly so that the inter-element spacing is fixed at half a wavelength at the operating frequency). It is assumed that in the downlink of the secondary network, the SBS transmits independent signals to $K$ users, one user at a time.

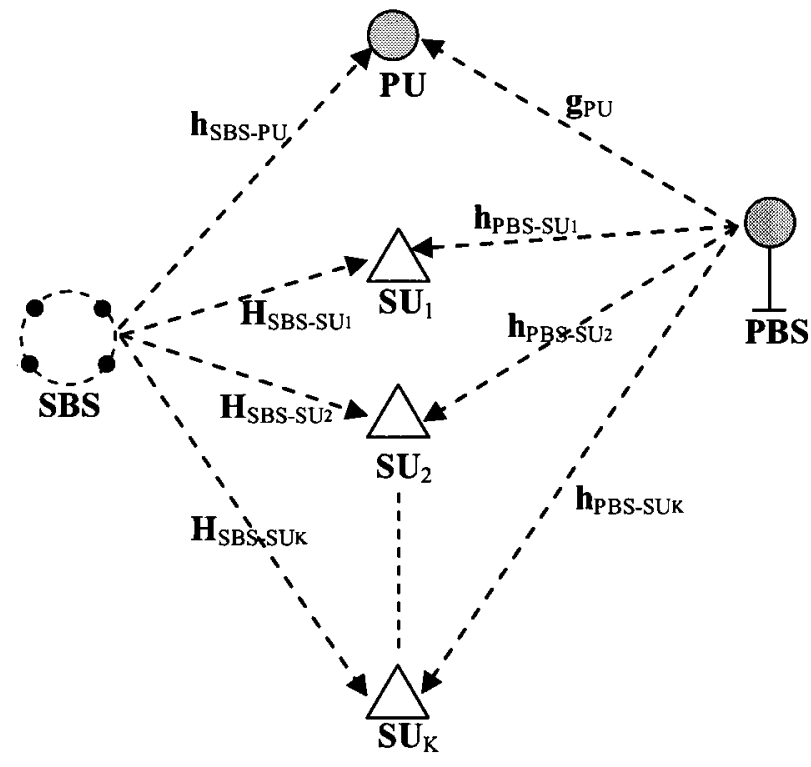

Figure 5.13. System model 
Using the WINNER channel model, the MIMO channel between the SBS and the $k t h \mathrm{SU}\left(H_{S B S-S U k}^{M \times N}\right)$, the MISO channels between the SBS and the PU $\left(h_{S B S-P U}^{1 \times N}\right)$, the single-input multiple-output (SIMO) channel between the PBS and the kth $S U$ $\left(h_{P B S-S U k}^{M \times 1}\right)$, and the path attenuation between the PBS and the PU $\left(g_{P U}\right)$ were synthesized. NLOS propagation conditions with 16 clusters, each of which consisted of 10 MPCs, were assumed [23]. Each MPC was characterized by its complex amplitude, delay, AOD and AOA. Consequently, the MIMO channel between the SBS and the kth SU was constructed as the sum of the MPCs, as in $[73,48]$ :

$$
H_{S B S-S U k}^{M \times N}=\sum_{l=1}^{L_{S B S-S U k}}\left\{\frac{\alpha_{l, S B S-S U k} e^{j \Phi_{l, S B S-S U k}}}{\sqrt{M N}}\left[\begin{array}{c}
e^{j k a_{S U k} \cos \left(\varphi_{r x, l, S B S-S U k}-\phi_{1, S U k}\right)} \\
e^{j k a_{S U k} \cos \left(\varphi_{r x, l, S B S-S U k}-\phi_{2, S U k}\right)} \\
\vdots \\
e^{j k a_{S U k} \cos \left(\varphi_{r x, l, S B S-S U k}-\phi_{M, ~ S U k}\right)}
\end{array}\right]\left[\begin{array}{c}
e^{j k a_{S B S} \cos \left(\varphi_{x, l, l, S B S-S U k}-\phi_{1, S B S}\right)} \\
e^{j k a_{S B S} \cos \left(\varphi_{t x, l, S B S-S U k}-\phi_{2, S B S}\right)} \\
\vdots \\
e^{j k a_{S B S} \cos \left(\varphi_{t x, l, S B S-S U k}-\phi_{N, S B S}\right)}
\end{array}\right]^{T}\right\}
$$

where $\phi_{n, S B S}$ is the angular position of the $n$-th element in the SBS array and is given by $\phi_{n, S B S}=2 \pi n / N, n=1, \ldots ., N ; \phi_{m, S U k}$ is the angular position of the $m$-th element in the $k t h$ SU array and is given by $\phi_{m, S U k}=2 \pi m / M, m=1, \ldots, M ; a_{S B S}$ and $a_{S U k}$ are the radii of the UCA at the SBS and the $k t h \mathrm{SU}$, respectively; $k=2 \pi / \lambda$ is the wavenumber at the operating frequency and $\lambda$ is the corresponding wavelength; $L_{S B S-S U k}$ is the total number of MPCs associated with the channel between the SBS and the kth SU; and $\alpha_{l, S B S-S U k}, \Phi_{l, S B S-S U k}, \varphi_{r x, l, S B S-S U k}$ and $\varphi_{t x, l, S B S-S U k}$ are the amplitude, phase, AOD, and AOA of the $l$-th such MPC. 
Similarly, the MISO channel between the SBS and the PU was constructed as:

$$
h_{S B S-P U}^{1 \times N}=\sum_{l=1}^{L_{S B S-P U}}\left\{\frac{\alpha_{l, S B S-P U} e^{j \Phi_{l, S B S-P U}}}{\sqrt{N}}\left[\begin{array}{c}
e^{j k a_{S B S} \cos \left(\varphi_{x, l, l, S B S-P U}-\phi_{1, S B S}\right)} \\
e^{j k a_{S B S} \cos \left(\varphi_{x, l, S B S-P U}-\phi_{2, S B S}\right)} \\
\vdots \\
e^{j k a_{S B S} \cos \left(\varphi_{x, l, L S B S-P U}-\phi_{N, S B S}\right)}
\end{array}\right]^{T}\right\}
$$

where $L_{S B S-P U}$ is the total number of MPCs associated with the channel between the SBS and the PU; and $\alpha_{l, S B S-P U}, \Phi_{l, S B S-P U}$, and $\varphi_{t x, l, S B S-P U}$ are the amplitude, phase, and AOD of the $l$-th such MPC.

Further, the SIMO channel between the PBS and the kth SU is written as:

$$
h_{P B S-S U k}^{M \times 1}=\sum_{l=1}^{L_{P S B-S U k}}\left\{\frac{\alpha_{l, P B S-S U k} e^{j \Phi_{l, P B S-S U k}}}{\sqrt{M}}\left[\begin{array}{c}
e^{j k a_{S U k} \cos \left(\varphi_{r x, l, P B S-S U k}-\phi_{1, S U k}\right)} \\
e^{j k a_{S U k} \cos \left(\varphi_{r, l, l, P B S-S U k}-\phi_{2, S U k}\right)} \\
\vdots \\
e^{j k a_{S U k} \cos \left(\varphi_{r, l, P B S-S U k}-\phi_{M, S U k}\right)}
\end{array}\right]\right\}
$$

where $L_{P B S-S U k}$ is the total number of MPCs associated with the channel between the PBS and the $k$ th SU; and $\alpha_{l, P B S-S U k}, \Phi_{l, P B S-S U k}$, and $\varphi_{r x, l, P B S-S U k}$ are the amplitude, phase, and AOA of the $l$-th such MPC.

Let $P_{P B S}, P_{S B S}$ denote the transmit power of the PBS, and the transmit power of the SBS, respectively, which are assumed to be equal (i.e., $P_{P B S}=P_{S B S}$ ). If the SBS transmits a data stream to the kth $\mathrm{SU}$, then the received signal at the $\mathrm{PU}$ is written as:

$$
y_{P U}=\sqrt{P_{S B S}} h_{S B S-P U}^{1 \times N}\left(w_{S B S-S U k}^{1 \times N}\right)^{H} s_{S U k}+\sqrt{P_{P B S}} g_{P U} s_{P U}+z_{P U}
$$

And, the received signal at the $k t h \mathrm{SU}$ is expressed as:

$$
y_{S U k}=\sqrt{P_{S B S}}\left(w_{S U k}^{1 \times M}\right)^{*} H_{S B S-S U k}^{M \times N}\left(w_{S B S-S U k}^{1 \times N}\right)^{H} s_{S U k}+\sqrt{P_{P B S}}\left(w_{S U k}^{1 \times M}\right)^{*} h_{P B S-S U k}^{M \times 1} s_{P U}+z_{S U k},
$$


where $w_{S B S-S U k}^{1 \times N}$ and $w_{S U k}^{1 \times M}$ are the beamforming weight vectors at the SBS and the $k$ th SU, respectively; $z_{P U}$ and $z_{S U k}$ are the zero-mean i.i.d. additive white Gaussian noise processes at the PU and the $k t h \mathrm{SU}$, where both of them have $\sigma^{2}$ variance; $s_{P U}$ and $s_{S U k}$ are the modulated signals for the $\mathrm{PU}$ and the $k t h \mathrm{SU}$, respectively.

When there is no transmission from the SBS, the SNR at the PU $\left(S N R_{P U}\right)$ is written as:

$$
S N R_{P U}=\frac{\left|g_{P U}\right|^{2} P_{P B S}}{\sigma^{2}}
$$

When the SBS is active (i.e., it transmits a data stream to the $k$ th $\mathrm{SU}$ ), the SINR at the PU ( $\left.\operatorname{SINR}_{P U}\right)$ is written as:

$$
\operatorname{SINR}_{P U}=\frac{\left|g_{P U}\right|^{2} P_{P B S}}{\left|h_{S B S-P U}^{1 \times N}\left(w_{S B S-S U K}^{1 \times N}\right)^{4}\right|^{2} P_{S B S}+\sigma^{2}}
$$

Consequently, the SINR at the $k t h$ SU ( $\left.S I N R_{S U k}\right)$ is given by:

$$
S I N R_{S U k}=\frac{\left|\left(w_{S U k}^{1 \times M}\right)^{*} H_{S B S-S U k}^{M \times N}\left(w_{S B S-S U k}^{1 \times N}\right)^{H}\right|^{2} P_{S B S}}{\left|\left(w_{S U k}^{1 \times M}\right)^{*} h_{P B S-S U k}^{M \times 1}\right|^{2} P_{P B S}+\sigma^{2}},
$$

and the associated rate of transmission to the $k t h$ SU is given by:

$$
C_{S U k}=\log _{2}\left(1+S I N R_{S U k}\right)
$$

It is clear from (5.21) and (5.22) that when the SBS transmits data to the kth SU, the resulting interference changes the SINR at the PU and, consequently, its data rate. Our goal here was to demonstrate a method that allows the SBS to transmit data to the SUs in such a way that the resulting interference does not affect the quality-of-service (QoS) provided to the PU. Following [82], the design criterion is to allow a specified maximum interference on the link to the PU, as defined by: 


$$
\gamma(d B)=S N R_{P U}(d B)-\operatorname{SINR}_{P U}(d B) \leq \gamma_{t h}(d B)
$$

where $\gamma_{t h r}(d B)$ is the maximum interference level that the PU can tolerate, and still offer the required QoS.

\subsubsection{The Frame Structure and Collaboration between Networks}

The following two assumptions were made regarding the frame structure used in the secondary network and the collaboration between the primary and the secondary networks.

1. There is a discovery window, consisting of $D$ minislots, in which the SBS sends $D$ test beams (one beam in each minislot) to the PU. The PU then estimates SINR values associated with each test beam and transmits back the index of the first beam tested that results in a SINR that meets its interference criterion.

2. For the secondary network, it is assume that every time slot of length $T$ consists of three parts:

a) One section of training minislots of length $\tau_{T}$ in which the SBS sends pilot signals to all the SUs and gets feedback regarding their channel conditions.

b) One delay minislot of length $\tau_{D}$ which represents the delay needed by the SBS to send test beams and receive feedback from the PU. The details of this task are explained in the next section.

c) The remaining time interval, $T-\tau_{T}-\tau_{D}$, is used for data transmission.

\subsubsection{The Cluster-Based Spatial Opportunistic Spectrum Sharing Technique for Cognitive Radio Systems}

The clustering nature of wireless channels was explained in Section 5.1. In this section, this tendency allows transmission from the SBS to the SUs in such a way that the interference caused to the PU is kept under a certain threshold. This can be done by discovering the locations of IOs that result in the strongest cluster paths (multipath routes) that link the SBS with the SUs and select the paths that are most spatially orthogonal with the radio link between the SBS and the PU. At the same time, the multi- 
antenna terminals at the SUs try to maximize their SINRs and consequently their rate by beamforming toward the illuminated IOs and attenuating the received signal from the PBS. This is done by adjusting the direction of their beamformers in such a way that their SINRs are maximized according to (5.23.). The coexistence of the PU and SU data transmissions in the same frequency band without violating the interference threshold at the PU terminal can be achieved as described below.

\subsubsection{Estimation of the AOD associated with the strongest clusters by the SUs}

In this step, each SU estimates the AOD associated with the strongest cluster of energy that it receives from the SBS, and its associated SINR. Each Tx antenna at the SBS transmits a pilot symbol from a set of mutually orthogonal signals. The $k t h$ SU does the following: First, it estimates the AOD (at the SBS) of the strongest received beam (i.e., $\left.\varphi_{t x, s t, S U k}\right)$. Then it uses the estimated $\varphi_{t x, s t, S U k}$ to perform joint $\mathrm{Tx}-\mathrm{Rx}$ beamforming towards the IO that generates the strongest cluster and estimates the best AOA (i.e., $\left.\varphi_{t x, s t, S U k}\right)$ that maximizes the received SINR. Finally, it estimates this SINR (i.e., $\left.S N I R_{\max , S U k}\right)$. It is assumed that each SU feeds back the $S N I R_{\max , S U k}$ and the $\varphi_{t x, s t, S U k}$ using a limited number of feedback bits. The details of these three steps are explained in the following:

\section{a) Angle-of-departure estimation}

If each Tx antenna at the SBS transmits a pilot symbol $p_{n}$ (where $p_{1}, p_{2}, \ldots, p_{N}$ is a set of mutually orthogonal signals), then, under the system setup explained in Section 5.4.2., the signal received by the $k t h \mathrm{SU}$ is given by

$$
y_{S U k}^{M \times 1}=\sqrt{P_{S B S}} H_{S B S-S U k}^{M \times N} s_{S U k}+\sqrt{P_{P B S}} h_{P B S-S U k}^{M \times 1} s_{P U}+z_{S U k}
$$

The relationship between the MIMO channel coefficients $H_{S B S-S U k}^{M \times N}$, the AODs at the SBS and the AOAs at the kth SU, of each MPC, is shown in (5.16.). 
Assume that the $N$ pilot signals transmitted by the SBS are known by all SUs, so that each SU can get an estimate of the channel matrix $H_{S B S-S U k}^{M \times N}$ from the received signal $y_{S U k}^{M \times 1}$. Given the limited number of antenna elements at the SBS and at the terminals of the SUs, only a limited number of beams $\left(B_{S U k}<<L_{S U k}\right)$ can be estimated, where each beam consists of several MPCs with close AODs and AOAs. Thus, (5.16.) can really only be estimated as:

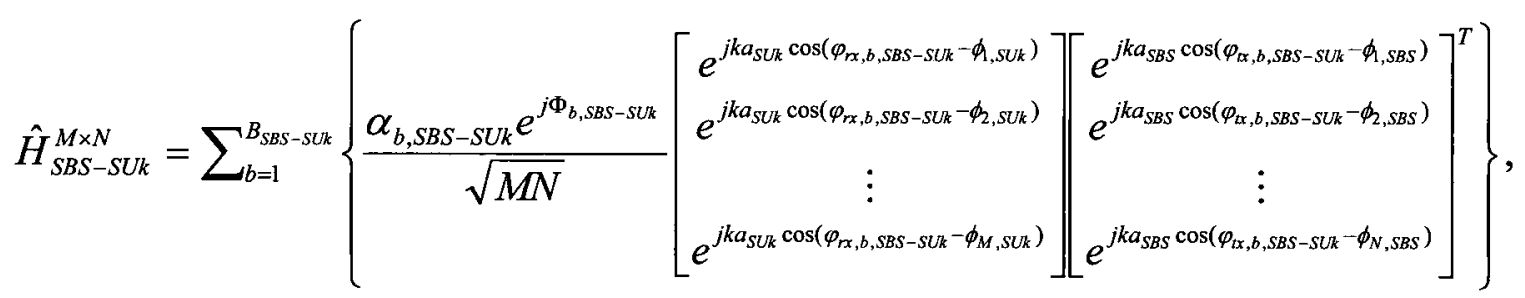

where $B_{S B S-S U k}$ is the total number of resolvable beams linking the SBS and the $k t h \mathrm{SU}$, and $\alpha_{b, S B S-S U k}, \Phi_{b, S B S-S U k}, \varphi_{t x, b, S B S-S U k}$ and $\varphi_{r x, b, S B S-S U k}$ are the amplitude, phase, AOD, and AOA of the $b$-th resolvable beam.

The information needed to estimate the AOD of the main beams is embedded in the coefficients of the estimated channel. The AOD of the strongest beam to the kth SU, $\left(\varphi_{t x, s t, S B S-S U k}\right)$ can be found by a simple beamscan [48]:

$$
\varphi_{t x, S t, S B S-S U k}=\arg \max _{0<=\varphi_{t x}<2 \pi} \sum_{m=1}^{M}\left|\underline{\hat{h}}_{m} S V_{\varphi_{t x}, S B S}^{H}\right|,
$$

where $|. .$.$| denotes absolute value, (. .)^{H}$ is the Hermitian transpose, $\hat{h}_{m}$ is the $m$-th row of $\hat{H}_{S B S-S U k}^{M \times N}$ (i.e., the estimated coefficients of the channel between the Tx elements at the SBS and the $m$-th antenna element at the $k t h$ SU), and $S V_{\varphi_{t x}, S B S}$ is the SBS element-space UCA steering vector in the AOD direction, which is defined as [73] 


$$
\left.S V_{\varphi_{x}, S B S}=(1 / \sqrt{N})\left[e^{j k a_{S B S} \cos \left(\varphi_{\varphi_{x}}-\phi_{, S B S S}\right)}, e^{j k a_{S B S} \cos \left(\varphi_{x}-\phi_{2, S B S}\right)}, \ldots \ldots ., e^{j k k_{S B S} \cos \left(\varphi_{\varphi_{x}}-\phi_{N}, S B S\right.}\right)\right]
$$

Note that to reduce the computational complexity associated with estimating $\varphi_{t, s t, S B S-S U k}$, the search in (5.28.) can be done by limiting the processing to only a single element of the antenna array at the $k t h$ SU.

\section{b) Joint Tx-Rx Beamforming}

Each SU estimates the AOA of the strongest cluster of energy, in addition to $\varphi_{t x, s t, S B S-S U k}$, which results from (5.28.), and performs a joint Tx-Rx beamforming in the direction of the IO that generates the strongest cluster at both the SBS and the SU terminals simultaneously, in such a way that maximizes its SINR (i.e., the SBS directs the transmitted energy towards the IO that generates the strongest cluster, and the SU forms a beam to maximize the received signal from SBS and, at the same time, reduce the interference from the PBS).

Given that an estimate of $h_{P B S-S U k}^{M \times 1}$ is available at the kth SU, the AOA of the strongest cluster received by it is found as:

$$
\varphi_{r x, s t, S B S-S U k}=\arg \max _{0<=\varphi_{r}<2 \pi}\left(\operatorname{SINR}_{S U k}\left[\varphi_{r x}\right]\right),
$$

where $\operatorname{SINR}_{S U k}\left[\varphi_{r x}\right]$ can be written as:

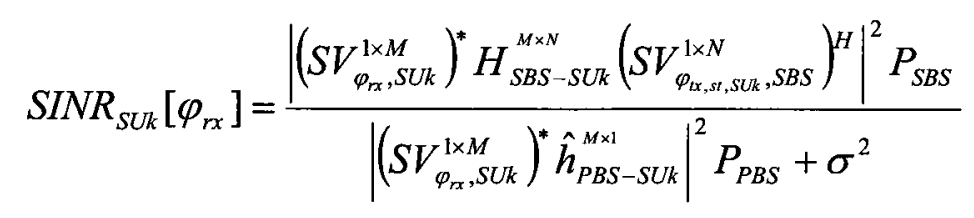

where $(. .)^{*}$ denotes the complex conjugate, $S V_{\varphi_{x, s, S S S K}, S B S}^{1 \times N}$ is the SBS element-space UCA steering vector of the strongest cluster associated with the $k t h S U$, and $S V_{\varphi_{r x}, S U k}^{1 \times M}$ is the element-space UCA steering vector at the $k t h S U$ in the $A O A$ direction, which is defined as [73]. 


$$
S V_{\varphi_{m}, S U k}^{1 \times M}=(1 / \sqrt{M})\left[e^{j k a_{S U k} \cos \left(\varphi_{r x}-\phi_{1, S U k}\right)}, e^{j k a_{S U k} \cos \left(\varphi_{r x}-\phi_{2, S U k}\right)}, \ldots \ldots . ., e^{j k a_{S U k} \cos \left(\varphi_{m x}-\phi_{M, S U k}\right)}\right]
$$

c) Estimating the Maximum SINR

The SINR associated with the strongest cluster received by the $k t h S U$, which results from the joint $T x-R x$ beamforming can be written as:

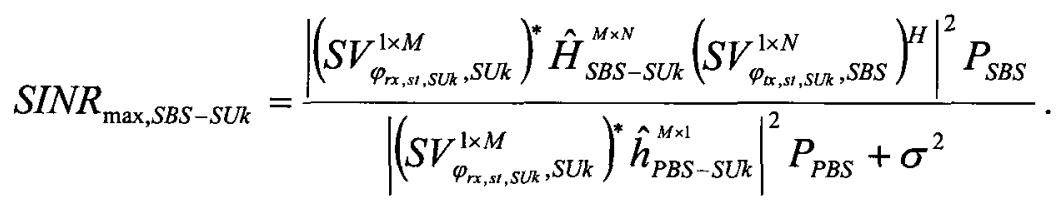

The computational complexity of estimating the AOD (at the SBS) of the strongest beam received by the $k t h \mathrm{SU}$ is a function of the number of antenna elements at the SBS and the step size of the beamscan search in (5.28.). For example, if each SU feeds back the AOD information using $x$ feedback bits, then a step size of $2 \pi / 2^{x}$ is used in (5.28.) and consequently $2^{x} . N$ complex multiplications, and almost the same number of complex additions, are required at each user terminal. Similarly, the computational complexity of estimating the AOA of the strongest beam received by the $k t h S U$, is a function of the number of antenna elements at the SU station and the step size of the beamscan search in (5.30.). However, the step size of the search in (5.30.) is not restricted by the feedback bits constraint. Instead, it depends solely on the computational power of the SU terminal.

\subsubsection{Transmission of Test Beams by the SBS}

In this step, the SBS transmits test beams and receives feedback from the PU regarding adhering (or not adhering) to the maximum-allowed interference threshold requirement. Using the feedback information, the SBS sorts the SUs according to their reported SINRs in a descending order and selects the $D$ SUs with the strongest SINRs. The SINR and the AOD of the $d t h$ selected SU are denoted as $\operatorname{SINR}_{d}$ and $\varphi_{t x, d}$, respectively. For each of these $D$ SUs, the SBS constructs a test beam such that it transmits the majority of its energy to this $\mathrm{SU}$ through the best available transmission 
path. The test beam associated with the $d t h \mathrm{SU}$ is formed using conventional beamforming, with the main beam pointed at $\varphi_{t x, d}$ (i.e., $S V_{\varphi_{x, d}, S B S}$ ), which is defined in (5.29).

Using a discovery window, which consists of $D$ minislots, the SBS sends the ordered test beams (i.e., the first beam is associated with the SU having the highest SINR, and the last one is associated with the SU having the lowest SINR). The PU examines the beams sequentially until it finds the first one (if such exists) that does not violate the maximum interference threshold requirements as shown in (5.25.); then it feeds back the index of this beam to the SBS.

\subsubsection{Transmission of the Data Stream}

In this step, the SBS transmits a data stream to the selected SU. If at least one of the test beams results in an SINR at the PU that meets the threshold requirement, the SBS uses its index (which was fed back from the PU) to send data to the associated SU. Assume that the kth $\mathrm{SU}$ is the selected $\mathrm{SU}$ for transmission, then its rate is given by:

$$
C_{S U k}=\frac{T-\tau_{T}-\tau_{D}}{T} \log _{2}\left(1+\operatorname{SINR}_{\max , S B S-S U k}\right),
$$

where $S I N R_{\max , S B S-S U k}$ is calculated from (5.33) by substituting $\hat{H}_{S B S-S U k}^{M \times N}$ and $\hat{h}_{P B S-S U k}^{M \times 1}$ by $H_{S B S-S U k}^{M \times N}$. and $h_{P B S-S U k}^{M \times 1}$, respectively.

\subsubsection{Simulation Results}

In this section, an evaluation of the throughput resulting from using the proposed algorithm is presented. The system model and the frame structure presented in sections 5.4.2. and 5.4.3. were used, and Monte Carlo simulations were conducted, with 5000 trials. It was assumed that: a) the estimation error on the $k t h \mathrm{SU}$ channel (for $H_{S B S-S U k}^{M \times N}$ and $h_{P B S-S U k}^{M \times 1}$ ) was modelled as a complex Gaussian random variable with $\sigma_{\text {error }}^{2}$ equal to $3 \%$ of the power in the impulse response estimated for the channel; b) the SBS has a 
UCA with 4-elements; c) the SUs have two possible UCA antenna configurations with 2elements and 4-elements; d) each SU uses 4 bits to feedback the AOD information and another 4 bits to feed back the SINR information; e) $\frac{P_{S B S}}{\sigma^{2}}=\frac{P_{P U}}{\sigma^{2}}=10 d B$; and f) $\tau_{T}+\tau_{D}=0.1 T$. It was further assumed that all the generated channels were independent and remained constant during the interval $T$.

Figure 5.14 shows the effect of increasing the number of the test beams sent by the SBS. It is assumed that it would be possible to use $2,4,8$, or 16 test beams, which would require 1, 2, 3 and 4 feedback bits, respectively, from the PU to indicate the successful beam (if such exists). It is clear that increasing the number of test beams increased the sum-capacity; however, the sum-rate increment per feedback bit decreased as a result of using more and more test beams.

Figure 5.15 shows the effect of using a higher SINR acceptability threshold. As expected, using a higher threshold (i.e., allowing more interference) increased the sumcapacity of the secondary network. 


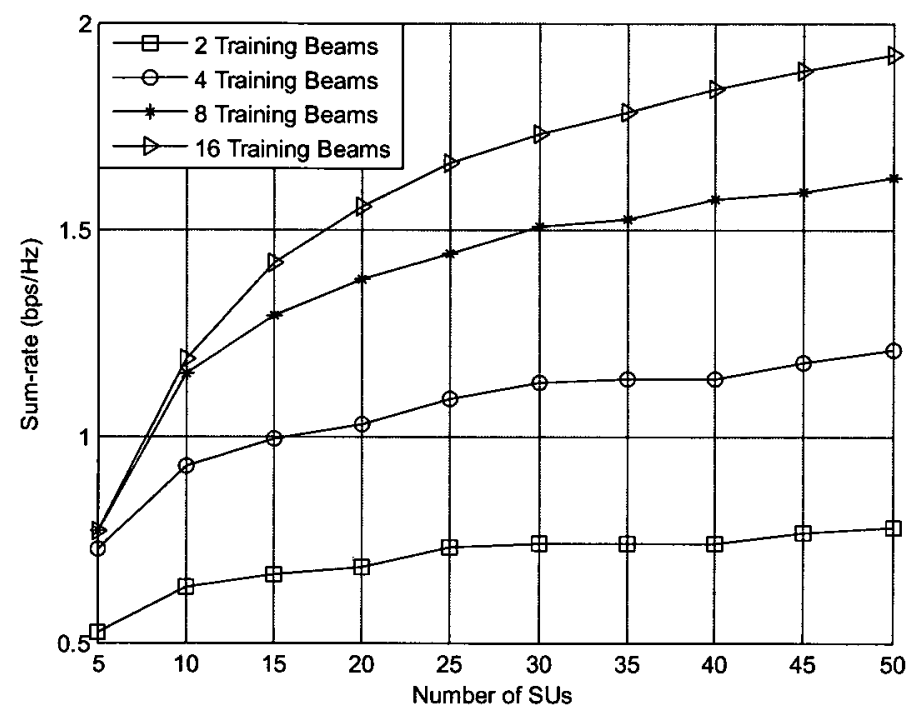

Figure 5.14. Effect of increasing the number of test beams on the sum capacity as a function of the total number of SUs. SBS 4 elements, SUs with 2 elements, $\quad \gamma_{t h}=0.5 d B, \quad\left(P_{S B S} / \sigma^{2}\right)=\left(P_{P U} / \sigma^{2}\right)=10 d B, \quad$ and $\left(\tau_{T}+\tau_{D}\right)=0.1 T$

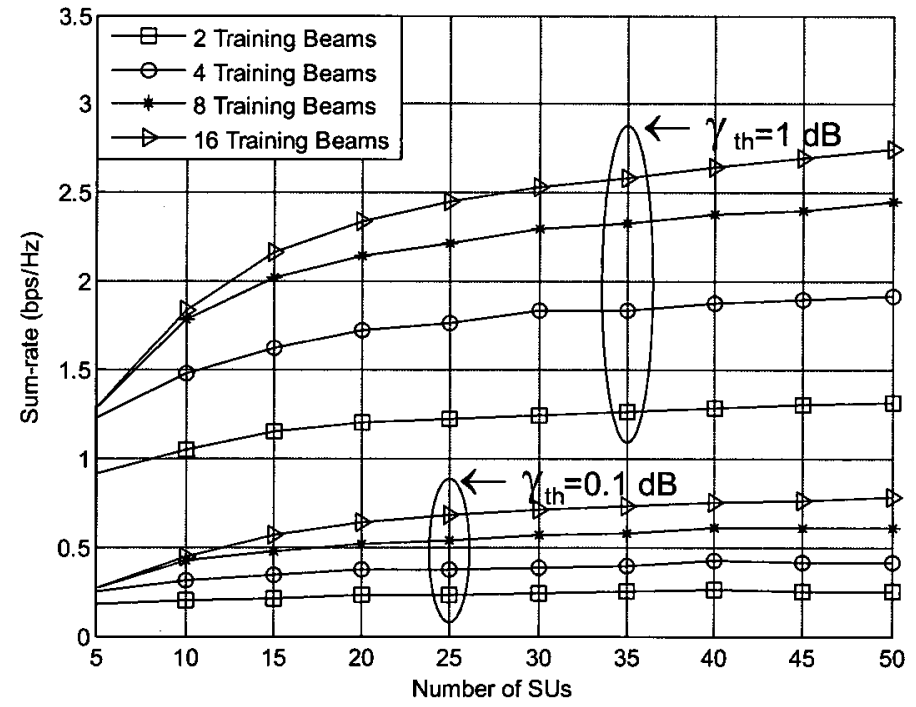

Figure 5.15. Effect of changing the maximum allowed interference $\left(\gamma_{t h}\right)$ on the sum capacity as a function of the total number of SUs. SBS 4 elements, SUs with 4 elements, $\left(P_{S B S} / \sigma^{2}\right)=\left(P_{P U} / \sigma^{2}\right)=10 d B$, and $\left(\tau_{T}+\tau_{D}\right)=0.1 T$. 


\subsection{Summary}

- A novel transmission technique for use on MIMO broadcasting channels has been introduced, in which the system takes advantage of multiuser diversity caused by the existence of clusters on wireless channels. In the proposed AOD-OSDMA technique, the BS transmits a set of mutually orthogonal signals that enables each user to estimate the AOD (at the BS) and the AOA (at the user station) of the strongest beam, perform joint $\mathrm{Tx}-\mathrm{Rx}$ beamforming, and estimate the corresponding SNR at the user station. The BS uses the reported AODs and SNRs to select a subgroup of users for data transmission in such a way that the sum capacity of the system is maximized. On microcellular channels simulated using the WINNER model, the proposed technique improves the sum capacity over that achievable when using OSDMA-S, regardless of the number of users (low, medium, or high) in both LOS and NLOS conditions. The main advantage of the proposed AOD-OSDMA is the non-random nature of the beam designing procedure, where the feedback information allows the BS to design the transmit beams for a selected group of users. Compared to OSDMA-S, the proposed AODOSDMA technique: (1) reduces the number of required directional information feedback bits by $33 \%$ and $55 \%$ with 4 and $8 \mathrm{Tx}$ antennas respectively, and (2) improves the throughput of the system by up to $30 \%$ with $4 \mathrm{Tx}$ antennas and up to $100 \%$ with $8 \mathrm{Tx}$ antennas, when users are equipped with a single antenna. In the case of users equipped with two antennas, the improvement of AOD-OSDMA over OSDMA-S is up to $50 \%$ and $160 \%$ with 4 and $8 \mathrm{Tx}$ antennas, respectively.

- A technique has been introduced that allows the coexistence of secondary users with a primary user, simultaneously in the same frequency band, in an underlying spectrum sharing setup. The proposed technique takes advantage of the existence of multipath clusters in wireless channels and utilizes the existence of clusters to allow the transmission from a SBS without violating a pre-determined interference threshold at the $\mathrm{Rx}$ of the PU. The proposed technique does not require full CSI. Instead, it efficiently implements joint $\mathrm{Tx}-\mathrm{Rx}$ beamforming by 
collecting a limited number of feedback bits from the secondary users, which are used to estimate the quality and directionality of the secondary users' channels, and from the primary user, to guarantee adherence to the allowed interference criterion. Simulations show that the proposed technique allows the achievement of reasonably high throughput, when evaluated using the WINNER channel model under urban micro-cellular NLOS propagation conditions. 


\section{CHAPTER 6:}

\section{EVALUATION OF THE COMMUNICATIONS PERFORMANCE ACHIEVABLE ON BASE STATION TO HANDHELD-TERMINAL RADIO LINKS WITH SEVERAL DIFFERENT PRACTICAL RECEIVE ANTENNA ARRAYS}

\subsection{Introduction}

The theoretical high capacity that MIMO systems can achieve is based on ideal assumptions, whereby the environment of operation is assumed to result in severe multipath propagation, and the cross-correlation among signals at different elements in $\mathrm{Rx}$ and $\mathrm{Tx}$ antenna arrays is assumed to be negligible [59]. However, not all operating environments can be characterized as being influenced by severe multipath propagation, and antenna array configuration, orientation, and element efficiency all have significant influence on signal cross-correlations. The theoretical effects of array configuration, array orientation, and the propagation environment on MIMO capacity have been studied by several researchers, using two approaches.

In the first approach, various different channel models were used to generate instantaneous MIMO channel matrices for the subsequent estimation of corresponding channel capacities. The channel models that were employed include the geometrically based single bounce model [88], the spatial channel model [89], and 3D spatial variant multi-ray radio wave propagation models [90].

In the second approach, empirical data were used to evaluate the performance of different receive antennas [91 - 94]. To evaluate link performance when using different multi-antenna systems, the work in $[92,93]$ considered only the mean effective gain, which is the SISO parameter often used in mobile radio work to evaluate the "effective gain" of an antenna in a multipath environment. In [94], new parameters were introduced for empirical characterization of MIMO antennas which quantify the capability of the associated MIMO transmit and received antennas to transfer energy and the capability of a MIMO link using specific antennas to offer diversity or spatial multiplexing gains. 
In this chapter, empirical information was used to evaluate the performance of different receive antennas. Such information was obtained through the analysis of 2.25 $\mathrm{GHz}$ propagation measurements recorded in a high-rise section of downtown Ottawa, as reported in Chapter 3. First, the parameters of MPCs that were estimated using the ISISAGE algorithm from the measurement data were used to construct a radio-channel matrix corresponding to each measurement snapshot. Then, during this work, the constructed matrices were used to study the influence on BS to handheld terminal (HT) radio-link performance of the position, orientation, and polarization of inverted-F antenna elements within a HT. More specifically, effective link gain, link matrix eigenvalue dispersion, and link outage capacity corresponding to different HT antenna configurations and different HT operating positions were estimated.

The rest of this chapter is organized as follows. Section 6.2. discusses the method used to synthesize the MIMO channel matrices for the communication system of interest at every street location (SL) where a snapshot of propagation data was recorded. Section 6.3. describes the estimation of effective link gain, eigenvalue dispersion and radio link capacities. Section 6.4. describes the HT operating positions and HT antenna configurations that were considered. Results, including a comparison of the above-cited channel-performance characteristics corresponding to the use of different antennaelement pairs in a HT for three different HT operating positions, are given in Section 6.5. Finally, a summary is included in Section 6.6.

\subsection{The Synthesis of MIMO Radio Link Matrices using the ISI-SAGE Extracted Parameters}

In order to study the influence on link performance in a hypothesized microcellular MIMO communication system of: (a) different dual inverted-F Rx antenna configurations, (b) antenna element efficiency, and (c) different HT operating positions, the complex signals that would appear at the terminals of the specified antennas at each 
SL where propagation measurements were recorded were estimated. ${ }^{1}$ To accomplish this, the complex amplitude, elevation AOA, azimuth AOA and delay of each MPC were used in plane-wave reconstructions. In addition, in the absence of available measurements, AODs were randomly selected for each MPC from realizations generated based on the assumption of a uniform distribution for AODs. It is considered that the assumption of this AOD distribution is as good as any for use in comparison to the relative merits of different receive antennas, when AOAs at the $\mathrm{Rx}$ are known from measurements. It is also considered that this assumption may even be valid in NLOS cases, where propagation through building and spaces between buildings, as well as around street corners, is possible. Based on reports from the literature [72] that elevation AODs in urban microcells are not large, the elevation AOD for all MPCs $\left(\theta_{t x, \ell}\right)$ was assumed to be fixed and equal to $\pi / 2$ (i.e., all MPCs depart the Tx antenna in the azimuth plane).

Using the ISI-SAGE extracted parameters and the assumptions of: a) a uniform distribution for the azimuth AODs of received MPCs; b) plane-wave propagation, whereby waves impinging on the $\mathrm{Rx}$ antenna can be approximated as having a planewave front; and c) narrowband operation, wherein the signal bandwidth is much smaller than the reciprocal of the time taken by a radio wave to propagate across the $\mathrm{Rx}$ antenna array, instantaneous channel matrices were realized as outlined below.

If the nth Tx antenna in the UCA at the BS of the hypothesized microcellular system transmitted a narrowband signal represented in complex baseband notation as $u(t)$, the received signal at the $m t h$ element in the $\mathrm{Rx}$ antenna could be represented as

$$
y_{m n}(\tau)=h_{m n}(\tau) * u(t)
$$

where $h_{m n}(\tau)$ represents the time-invariant SISO impulse response of the radio link between the nth $\mathrm{Tx}$ antenna element and the $m t h \mathrm{Rx}$ antenna element, and * represents

\footnotetext{
1 Proximity effects due to the presence of a human operator were omitted from consideration.
} 
complex convolution. If $\mathrm{L}$ denotes the total number of received MPCs, the complex envelope of the corresponding received signal $y_{m n}$ can be written as [50]:

$$
y_{m n}=\sum_{\ell=1}^{L}\left\{\begin{array}{c}
\alpha_{\ell} \times v_{t x}\left(\theta_{t x, \ell}, \varphi_{t x, \ell}\right)[n] \times g_{t x}\left(\theta_{t x, \ell}, \varphi_{t x, \ell}\right)[n] \\
\times v_{r x}\left(\theta_{r x, \ell}, \varphi_{r x, \ell}\right)[m] \times F_{\vec{z}}\left(\theta_{r x, \ell}, \varphi_{r x, \ell}\right)[m] \times u\left(t-\tau_{\ell}\right)
\end{array}\right\}
$$

in which:

- $\alpha_{\ell}$ represents the complex amplitude of the $\ell$ th MPC,

- $v_{t x}\left(\theta_{t x, \ell}, \varphi_{t x, \ell}\right)[n]$ represents the $n t h$ element of the Tx antenna steering vector corresponding to the $\ell$ th $\mathrm{MPC}$,

- $g_{t x}\left(\theta_{t x, \ell}, \varphi_{t x, \ell}\right)[n]$ represents the gain of the $n t h \mathrm{Tx}$ antenna element in the $\left(\theta_{t x, \ell}, \varphi_{t x, \ell}\right)$ direction,

- $v_{r x}\left(\theta_{r x, \ell}, \varphi_{r x, \ell}\right)[m]$ represents the $m t h$ element of the $\mathrm{Rx}$ antenna steeringvector corresponding to the $\ell$ th $\mathrm{MPC}$, and

- $F_{\theta}\left(\theta_{r x, \ell}, \varphi_{r x, \ell}\right)[m]$ represents the vertically polarized component of the complex electromagnetic field pattern of the $m$ th $\mathrm{Rx}$ antenna element in the direction $\left(\theta_{r x, \ell}, \varphi_{r x, \ell}\right)$, corresponding to the $\ell$ th MPC, and is calculated as:

$$
F_{\bar{\theta}}\left(\theta_{r x, \ell}, \varphi_{r x, \ell}\right)[m]=\left\{\begin{array}{c}
\frac{E_{\theta}\left(\theta_{r x, \ell}, \varphi_{r x, \ell}\right)[m]}{\mid E_{\theta}\left(\theta_{r x, \ell}, \varphi_{r x, \ell}\right)[m]} \times \\
\sqrt{E f f[m] \times D_{r x}\left(\theta_{r x, \ell}, \varphi_{r x, \ell}\right)[m]} \times \sin \left(\theta_{r x, \ell}\right)
\end{array}\right\}
$$

in which;

- $D_{r x}\left(\theta_{r x, \ell}, \varphi_{r x, \ell}\right)[m]$ represents the directivity of the $m t h$ element of the $\mathrm{Rx}$ antenna in the $\left(\theta_{r x, \ell}, \varphi_{r x, \ell}\right)$ direction, corresponding to the $\ell$ th $\mathrm{MPC}$, 
- $\quad E f f[m]$ represents the sum of the mismatch and radiation efficiencies, or total efficiency of the mth element in the Rx antenna array, and

- $E_{\theta}\left(\theta_{r x, \ell}, \varphi_{r x, \ell}\right)[m]$ represents the vertically polarized component of the $\mathrm{E}$ field of the $m t h \mathrm{Rx}$ antenna element in the direction $\left(\theta_{r x, \ell}, \varphi_{r x, \ell}\right)$, corresponding to the $\ell$ th $\mathrm{MPC}$.

Since the work herein concerns the evaluation of different $\mathrm{Rx}$ antennas, it is assumed that all $\mathrm{Tx}$ antenna elements have an omnidirectional radiation pattern in the horizontal plane, and that all received MPCs departed from the Tx array in the horizontal plane. The gain of each Tx antenna element, $g_{t x}\left(\theta_{t x, \ell}, \varphi_{t x, \ell}\right)[n]$ was set therefore equal to unity, for all $\ell$ and $n$. The $\mathrm{Rx}$ antenna efficiencies were obtained during in-situ measurements at the terminals of antennas in a mock HT in an anechoic chamber. Radiation patterns that account for mutual coupling were obtained via electromagnetic simulations.

From (6.2) and (6.3) it is obvious that $h_{m n}$ can be constructed as:

$$
h_{m n}=\sqrt{E f f[m]} \times \sum_{\ell=1}^{L}\left\{\begin{array}{l}
\alpha_{\ell} \times v_{t x}\left(\theta_{t x, \ell}, \varphi_{t x, \ell}\right)[n] \times g_{t x}\left(\theta_{t x, \ell}, \varphi_{t x, \ell}\right) \times v_{r x}\left(\theta_{r x, \ell}, \varphi_{r x, \ell}\right)[m] \\
\times \frac{E_{\theta}\left(\theta_{r x, \ell}, \varphi_{r x, \ell}\right)[m]}{\left|E_{\theta}\left(\theta_{r x, \ell}, \varphi_{r x, \ell}\right)[m]\right|} \times \sqrt{D_{r x}\left(\theta_{r x, \ell}, \varphi_{r x, \ell}\right)[m]} \times \sin \left(\theta_{r x, \ell}\right)
\end{array}\right\} .
$$

An $M \times N$ MIMO matrix for any BS-HT link in the hypothesized communication system can then be written as:

$$
\boldsymbol{H}=\left[\begin{array}{cccc}
\boldsymbol{h}_{11} & \boldsymbol{h}_{12} & \cdots & \boldsymbol{h}_{1 N} \\
\boldsymbol{h}_{21} & \boldsymbol{h}_{22} & \cdots & \boldsymbol{h}_{2 N} \\
\vdots & \vdots & \ddots & \vdots \\
\boldsymbol{h}_{M 1} & \boldsymbol{h}_{M 2} & \cdots & \boldsymbol{h}_{M N}
\end{array}\right]
$$


As explained previously, to obtain values for $\varphi_{t x, \ell}$ for use in (6.4), it was assumed AODs at the BS of the hypothesized microcellular system had a uniform probability distribution in azimuth and were confined to the horizontal plane. A value for $\varphi_{t x, \ell}$ was thus selected for each MPC, for each data snapshot, from a pool of values generated randomly in accordance with a uniform distribution.

\subsection{Estimation of MIMO Link Performance in the Hypothesized System}

MIMO radio-links performance in a hypothesized communication system having a fixed microcellular BS and a HT moving throughout the environment, where the propagation measurements were made, were predicted. Specifically, effective link gain, $\left(\mathrm{G}_{\mathrm{ant}}\right)$, eigenvalue dispersion $\left(\mathrm{G}_{\mathrm{mux}}\right)$, and outage capacity $\left(C_{O}\right)$ on BS-HT MIMO radio links, (between the locations where the channel sounder $\mathrm{Tx}$ was set up during the propagation measurements and SLs where a propagation measurement snapshot was recorded), we estimated. The estimated values of $C_{O}$, reported later, apply to a situation as if there were perfect power control in the system under study.

$\mathrm{G}_{\text {ant }}$ is defined in [94], and can be estimated as

$$
G_{a n t}^{(i)}=\frac{\left\|H^{(i)}\right\|_{F}^{2}}{\left\|H_{i s o, s i i}^{(i)}\right\|_{F}^{2}},
$$

where,

$$
\left\|H_{i s o, s l i}^{(i)}\right\|_{F}^{2}=\frac{1}{2 N_{s n}+1} \sum_{i-N_{s n}}^{i+N_{s n}}\left\|H_{i s o}^{(i)}\right\|_{F}^{2},
$$

and $H^{(i)}$ represents the channel matrix defined in (6.5) by using values for $h_{m n}$ from (6.4) evaluated using the parameters applicable to the antennas proposed for use in the hypothetical system under study, and $H_{i s o}^{(i)}$ represents the same matrix evaluated using the $h_{m n}$ applicable when isotropic radiators are used for the elements of the HT and BS antennas. The additional subscript " $s l i$ " in the denominator denotes the fact that the 
denominator is a mean over some sliding spatial interval, where $2 N_{s n}+1$ is the number of propagation measurement snapshots recorded over the specified sliding window. The mean effective link gain (MELG) is defined as the mean of all the realizations of $G_{a n t}^{(i)}$.

Eigenvalue dispersion, symbolized as $G_{m u x}$, is defined in [94] as

$$
G_{m u x}^{(i)}=\frac{\left(\prod_{k=1}^{K} \lambda_{k}^{(i)}\right)^{1 / K}}{\frac{1}{K} \sum_{k=1}^{K} \lambda_{k}^{(i)}},
$$

where $\lambda_{k}^{(i)}$ represents the $k t h$ eigenvalue of $H^{(i)}$. This parameter characterizes the spread, or range of eigenvalues of $H^{(i)}$, as being close to unity if the spread is small, and close to zero if the spread is large. A large eigenvalue spread indicates a significant imbalance in the gains of virtual parallel transmission channels on a MIMO link. Alternately, a small eigenvalue spread can lead to significant diversity or multiplexing gains as a result of the existence of a number of virtual parallel channels with nearly equal gains.

MIMO link capacity is expressed as

$$
C_{H}^{(i)}=\log _{2}\left|I+\frac{\rho}{N} \frac{H^{(i)} H^{(i) H}}{P_{n o r m}}\right|,
$$

where $\rho$ is the SNR on the radio link, $N$ represents the number of transmit antennas. Normalisation by the factor $P_{\text {norm }}$ eliminates the influence of arbitrary Tx powers used during the propagation experiments and mitigates the influence of large area fading. This normalisation allows the isolated study of the effects on MIMO link performance of multipath dispersion, which causes decorrelation among the signals on different MIMO link antennas. Normally, the normalization is performed using link matrices that are constructed assuming the use of antennas of the same type as those selected for the communication system under study. Herein, however, in order to allow the comparison of MIMO link performance with different real-world antenna configurations, a common reference is used for normalization, regardless of the type of antennas selected for use in the system under study. This reference is calculated according to 


$$
P_{n o r m}=\frac{1}{M N}\left\|H_{i s o, s l i}^{(i)}\right\|,
$$

where $M$ and $N$ represents the number of receive and transmit antennas, respectively. This permits the study of the relative merits of different antenna types. In addition, since this normalization does not always result in a total received power of unity (i.e., a channel matrix with a fixed Forbenius norm), it permits the study of the influence on MIMO link performance of local received signal power variations (short-term fading); such variations typical on real-world radio channels. However, it should be noted that, since the normalisation averages out the influence of longer-term shadow fading, the specified SNR on a link to a specific SL would not be achieved unless there was power control in the hypothetical system under study. Values estimated herein for capacity would be overestimates, without power control.

\subsection{Antenna Configurations and HT Operating Positions that were Considered}

The BS antenna array was assumed to be a 4-element UCA with inter-elementspacing $=\lambda$. At the $\mathrm{Rx}$ a dual inverted-F antenna placed in one of 3 different configurations in a HT was considered. Three HT operating positions were considered, and are referred to as: (1) the Talk Position (TP), the Data Position (DP), and the Vertical Position (VP). These three positions are depicted pictorially in Figure 6.1. For each of these positions, three different $\mathrm{HT}$ antenna configurations were considered, including:

(1) The Bottom, Co-polarized (BC) configuration, in which two inverted-F antennas are located at the bottom of the HT, and arranged in co-polarized manner.

(2) The Bottom, Cross-polarized (BX) configuration, in which two inverted-F antennas are located at the bottom of the HT, but arranged in a cross-polarized manner.

(3) The Opposite, Cross-polarized (OX) configuration, in which one inverted-F antenna is located at the bottom of the HT and the second inverted-antenna is located at the top of the HT, and they are arranged in a cross-polarized manner. 
For each case, the total efficiencies (i.e., the sum of mismatch and radiation efficiencies) of the antenna elements were assigned one of three combinations of values, including

Combination 1: eff $_{1}=60 \%$ and eff $_{2}=60 \%$ (balanced),

Combination 2: eff $_{1}=40 \%$ and eff $_{2}=75 \%$ (unbalanced), and

Combination $3: \mathrm{eff}_{1}=75 \%$ and eff $_{2}=40 \%$ (unbalanced).

A total of 27 cases were therefore studied, resulting from the different combinations of HT positions ( 3 cases), antenna configurations ( 3 cases) and antenna efficiencies ( 3 cases). 


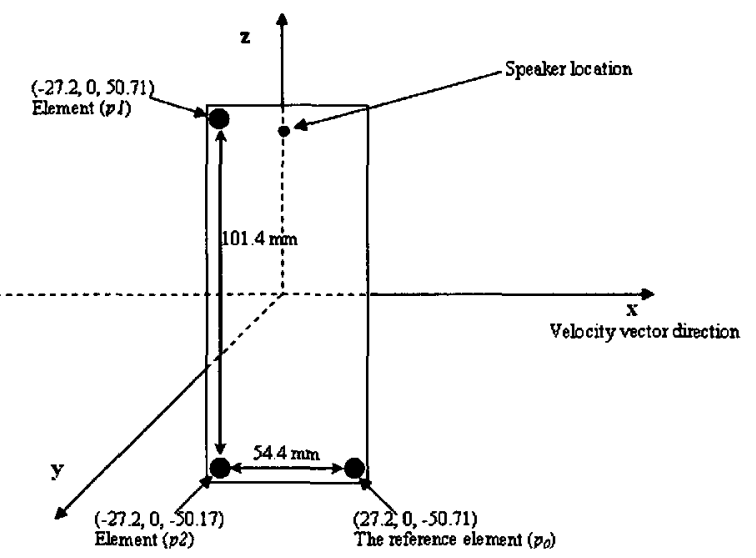

(a)

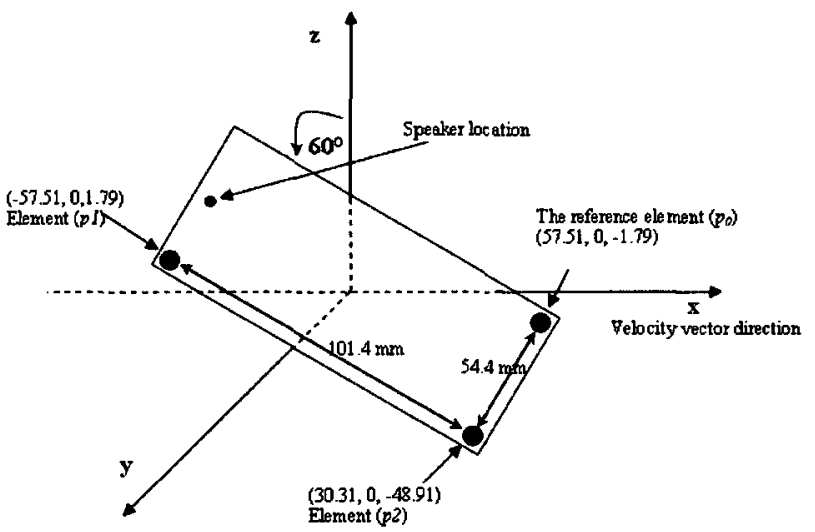

(b)

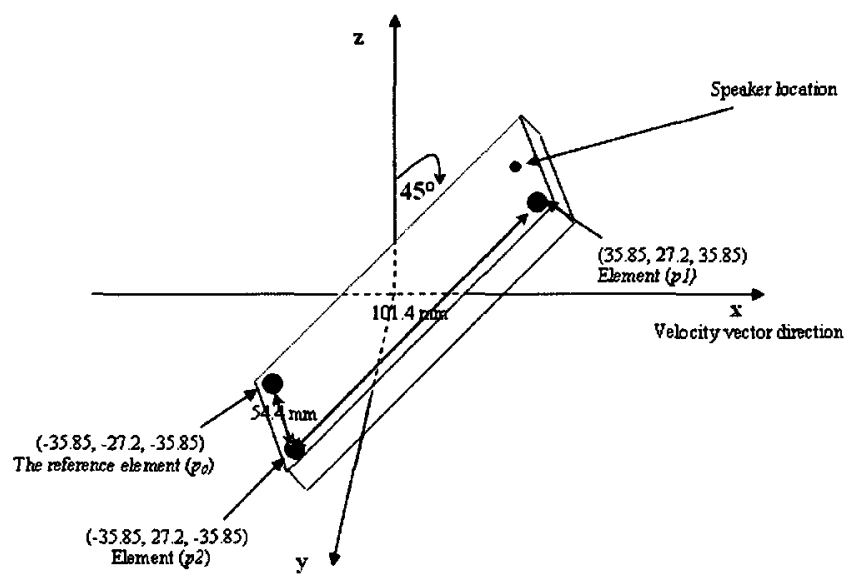

(c)

\section{Notes:}

The "Speaker location" point is a fictitious point used to define the relative locations of the different elements.

For the $\mathrm{BC}$ and $\mathrm{BX}$ configurations, elements $p_{0}$ and $p_{2}$ are used. For the OX configuration, elements $p_{0}$ and $p_{1}$ are used. $p_{0}$ is denoted as the "reference element" since its location is used in all configurations. The efficiency of the $p_{0}$ element is denoted by eff $I$ and the efficiency of the other element (whither $p_{1}$ or $p_{2}$ ) is denoted by eff 2 .

Figure 6.1. The relative locations of the different antenna elements in $3 D$ at the three different positions: (a) VP, (b) TP, and (c) DP. 


\subsection{Results}

A total of 15,000 propagation measurement snapshots were analyzed to synthesize a $4 \times 2$ MIMO link matrix for each, as described in foregoing sections. These matrices were then used to estimate $G_{a n t}^{(i)}$, and $G_{m u x}^{(i)}$ corresponding to location $i$, and, subsequently outage capacities, defined below, for a range of average microcell system SNRs. The propagation measurement snapshots that were analyzed included time series of 1000 consecutive snapshots from 15 NLOS CSMS routes of travel in the coverage area within two city blocks of the location where the channel sounder Tx was set up. Average system SNR values were varied from $0 \mathrm{~dB}$ to $40 \mathrm{~dB}$ in steps of $5 \mathrm{~dB}$; for each $\mathrm{HT}$ antenna Configuration/Position/Efficiency/SNR (C-P-E-SNR) combination. The normalization parameter $P_{n o r m}$ that was needed to calculate $\left\{G_{a n t}^{(i)}\right\}_{i=1}^{N_{s}}$ and $\left\{C_{H}^{(i)}\right\}_{i=1}^{N_{s}}\left(N_{s}\right.$ is the total number of processed snapshots) was estimated using a sliding window length of 101 snapshots (window length $=2 N_{s n}+1$ with $N_{s n}=50$ ). Considering the speed of the CSMS (between 5 and $10 \mathrm{mps}$ ) and the snapshot recording rate (250 snapshots per second), this sliding window would have been between $15 \lambda$ and $30 \lambda$ long. $^{2}$

Using data from each propagation measurement snapshot, for each of the C-P-ESNR combinations, $G_{a n t}^{(i)}$ and $C_{H}^{(i)}$ were estimated using different HT orientations ranging over 360 degrees in steps of 10 degrees.

\footnotetext{
${ }^{2}$ Due to the use of a sliding window of 101 snapshots, the number of values for $G_{a n t}^{(i)}$ and $C_{H}^{(i)}$ that were estimated was 13,500 .
} 
TABLE 6.1. is an example of results (outage capacities) from the analysis of one C-P-E-SNR combination. The columns represent the index of the estimated capacity realization. The rows represent the different orientations of the HT. For each snapshot $x$ and HT position $y$, the capacity $C_{x, y}$ is calculated. Thus, results for all C-P-E-SNR combinations include 36 orientations $\times 13,500$ sliding window lengths, or 486,000 values.

TABLE 6.1. Example of Results (outage capacities) from the Analysis of One C-P-ESNR Combination

\begin{tabular}{|c|c|c|c|c|c|c|c|c|c|c|}
\hline & \multicolumn{9}{|c|}{ Index of the measurement snapshot } \\
\hline & & 1 & 2 & $\ldots$ & $\ldots$ & $\ldots$ & $\ldots$ & $\ldots$ & 13499 & 13500 \\
\hline \multirow{9}{*}{ 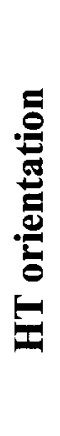 } & $\mathbf{0}^{\circ}$ & $\mathrm{C}_{1,0}$ & $\mathrm{C}_{2,0}$ & & & & & & $\mathrm{C}_{13499,0}$ & $\mathrm{C}_{13500,0}$ \\
\hline & $10^{\circ}$ & $\mathrm{C}_{1,10}$ & $\mathrm{C}_{2,10}$ & & & & & & $\mathrm{C}_{13499,10}$ & $\mathrm{C}_{13500,10}$ \\
\hline & $20^{\circ}$ & $\mathrm{C}_{1,20}$ & $\mathrm{C}_{2,20}$ & & & & & & $\mathrm{C}_{13499,20}$ & $\mathrm{C}_{13500,20}$ \\
\hline & $30^{\circ}$ & $\mathrm{C}_{1,30}$ & $\mathrm{C}_{2,30}$ & & & & & & $\mathrm{C}_{13499,30}$ & $\mathrm{C}_{13500,30}$ \\
\hline & .. & $\ldots$ & $\ldots$ & & & & & & $\ldots$ & $\ldots$ \\
\hline & .. & $\ldots$ & $\ldots$ & & & & & & $\ldots$ & $\ldots$ \\
\hline & .• & $\ldots$ & $\ldots$ & & & & & & $\ldots$ & $\ldots$ \\
\hline & $340^{\circ}$ & $\mathrm{C}_{1,340}$ & $\mathrm{C}_{2,340}$ & & & & & & $\mathrm{C}_{13499,340}$ & $\mathrm{C}_{13500,340}$ \\
\hline & $350^{\circ}$ & $\mathrm{C}_{1,350}$ & $\mathrm{C}_{2,350}$ & & & & & & $\mathrm{C}_{13499350}$ & $\mathrm{C}_{13500350}$ \\
\hline
\end{tabular}

All results from this work, exemplified by those in TABLE 6.1., will be of one of the following 4 types:

Type 1 results (all values): The empirical distribution of all the $36 \times 13,500$ link performance estimates (one for each HT orientation, for each propagation-measurement snapshot) is reported for each C-P-E-SNR combination.

Type 2 results (average over orientation): The empirical distribution of 13,500 link performance estimates is reported for each C-P-E-SNR combination. Each of these is associated with a single propagation-measurement snapshot; each was calculated by averaging performance estimates for $36 \mathrm{HT}$ orientations under the multipath conditions estimated from this snapshot. 
Type 3 results (the best orientation in each snapshot): The empirical distribution of 13,500 link performance estimates is reported for each C-P-E-SNR combination. Each of these values is associated with a single propagation measurement snapshot; each is calculated as the maximum value of the $\mathbf{3 6}$ link performance estimates corresponding to multipath conditions estimated from this snapshot. .

Type 4 results (the worst orientation in each snapshot): The empirical distribution of 13,500 link performance estimates is reported for each C-P-E-SNR combination. Each of these values is associated with a single propagation measurement snapshot; each is calculated as the minimum value of the 36 performance estimates, given the multipath conditions estimated from this snapshot.

\subsubsection{The Influence of Antenna Configuration, Antenna Efficiencies, and HT Position on $G_{\text {ant }}$}

Results in Figure 6.2. show that when the HT is in the vertical operating position, the antenna configurations that have both inverted-F elements at the bottom of the HT yield greater values of $G_{a n t}$ than the OX configuration, in which one antenna is at the bottom of the HT and one is at the top, and the two have different polarizations. Because of the large number of samples involved in the construction of the experimental cumulative probability distribution functions (ECDFs) shown, there is likely a very small region around each curve in which the true CDF must lie. However, given the presence of noise in the measured data and other approximations used in the analyses, one should not conclude from these results that there is a statistically-significant difference between the $\mathrm{BC}$ results and the BX results. Finally, note that there is some indication in all three sets of results in Figure 6.2. that $G_{a n t}$ is always greatest when one of the antenna elements is more efficient than the other. 
Similar results for the DP and TP of the HT can be seen in Figures 6.3., and 6.4. In all these cases, the values of $G_{a n t}$ are also greatest when the $H T$ antennas are at the bottom. In all cases, at least at the lower tails of the distributions (i.e., in less than about $30 \%$ of SLs) link gains seem to be slightly greater in cases where the bottom antennas are cross-polarized. In the DP, values of $G_{\text {ant }}$ are close to the greatest when the two antenna elements are equally efficient. However, when the HT is in the Talk, there is a greater disparity between such values when the antenna efficiencies are the same, and the best of the two values of $G_{\text {ant }}$ results when the antenna efficiencies are not equally efficient. It seems, therefore that if a "favoured" antenna can be identified, it will be the more efficient one. However, when such identification cannot be made, it is best that the antenna elements are equally efficient, since gains are lowest when the "wrong" antenna element is the most efficient.

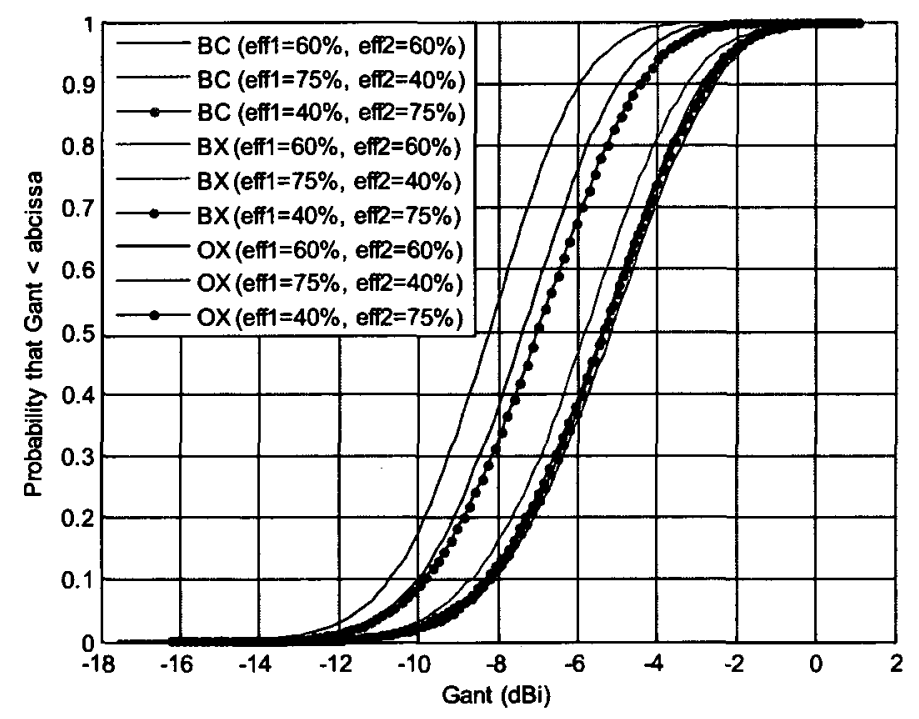

Figure 6.2. ECDF of $G_{a n t}$ at the VP for different configurations and element efficiencies combinations. 


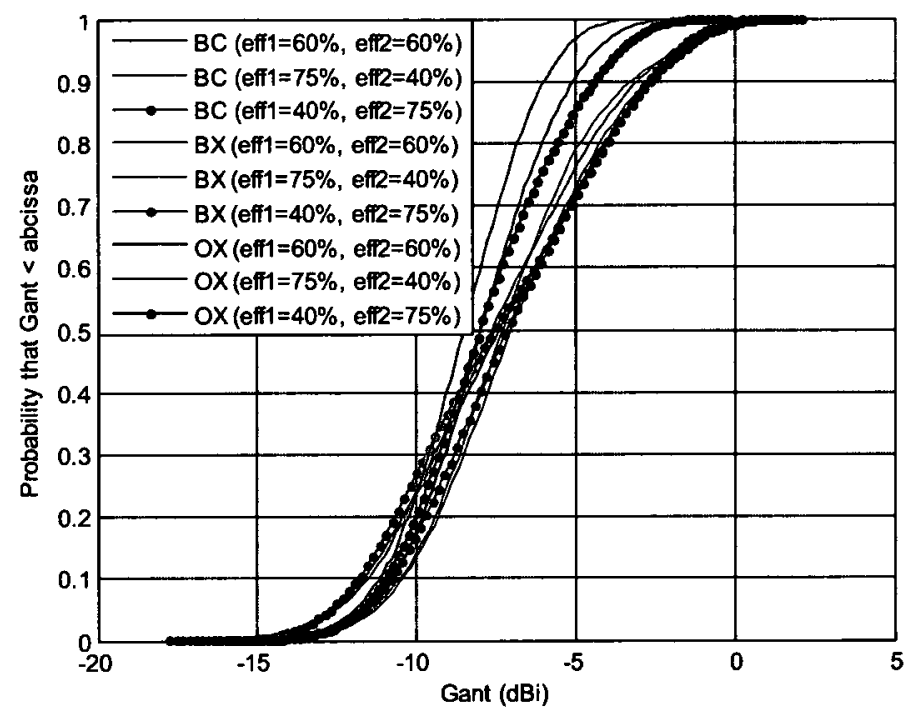

Figure 6.3. ECDF of $G_{a n t}$ at the DP for different configurations and element efficiencies combinations.

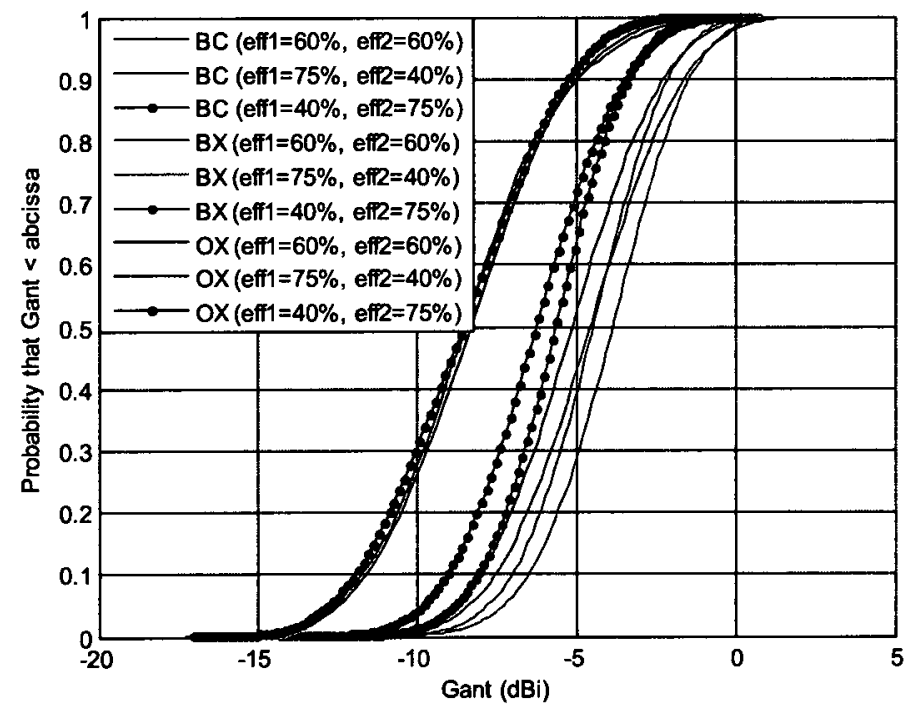

Figure 6.4. ECDF of $G_{a n t}$ at the TP for different configurations and element efficiencies combinations.

The above paragraph reports that there is some indication that values of $G_{a n t}$ are greatest for the BX antenna configuration. The relationship between the different antenna patterns and these link gains can be explained as follows. 
Figure 6.5. shows the power azimuth spectrum that was estimated from all 15,000 of the propagation measurement snapshots. From this figure it is easy to identify the street-canyon effect, which resulted in the arrival of most of the received power from 0 deg and 180 deg with respect to the velocity vector of the CSMS. However, a significant amount of power was still received from all other directions. This spectrum of power reception throughout all angles in azimuth suggests that if both of the receive antennas happen to have high gain in the directions of the strongest received signals (i.e., 0 or 180 $\mathrm{deg}$ ), then $\mathrm{G}_{\text {ant }}$ would always have positive values. However, considering practical issues such as the random orientation of a HT when it is used and the fact that its antennas have different pattern shapes directed in different directions, the probability of positive values for $G_{\text {ant }}$ is low, as reflected in Figures 6.2., 6.3., and 6.4. In fact, $G_{a n t}$ increases as the patterns of the antenna elements become broader, and decreases as the patterns become narrower (hence, receiving energy from fewer MPCs).

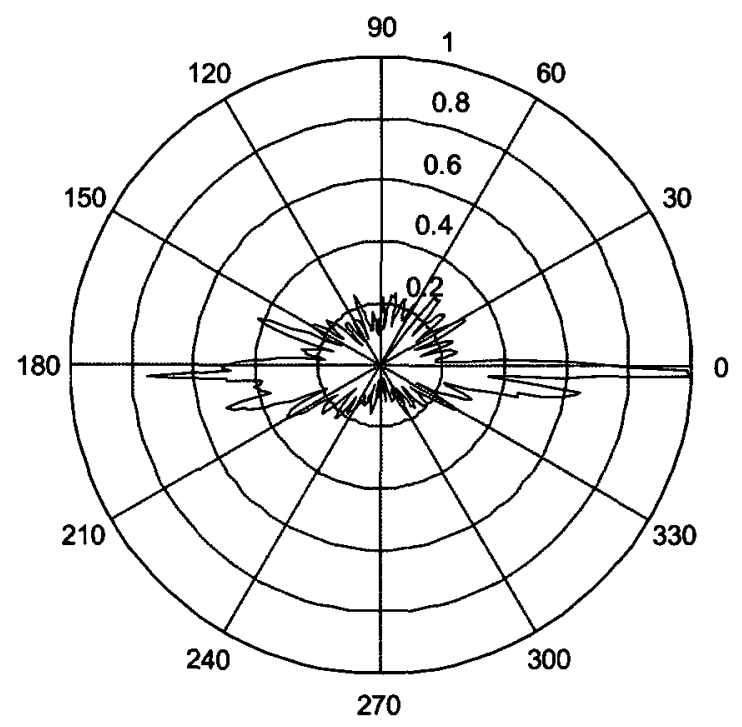

Figure 6.5, Power Azimuth Spectrum estimated using MPC parameters from all 15,000 propagation measurement snapshots. 
The foregoing can be verified through the examination of antenna patterns. Propagation measurement results indicate that most power at the CSMS would have been received at angles close to the horizontal plane (close to $\theta=\pi / 2$ ). Figure 6.6. shows the patterns, at $\theta=\pi / 2$, of the two HT antenna elements for the different configurations/positions. Upon comparing subplots (c), (f) and (i) in Figure 6.6., one observes that patterns for the BX antenna-element configuration have the least directivity. The antenna configuration $\mathrm{OX}$ results in patterns with the highest directivity, which yields the lowest values of $\mathrm{G}_{\text {ant }}$. 


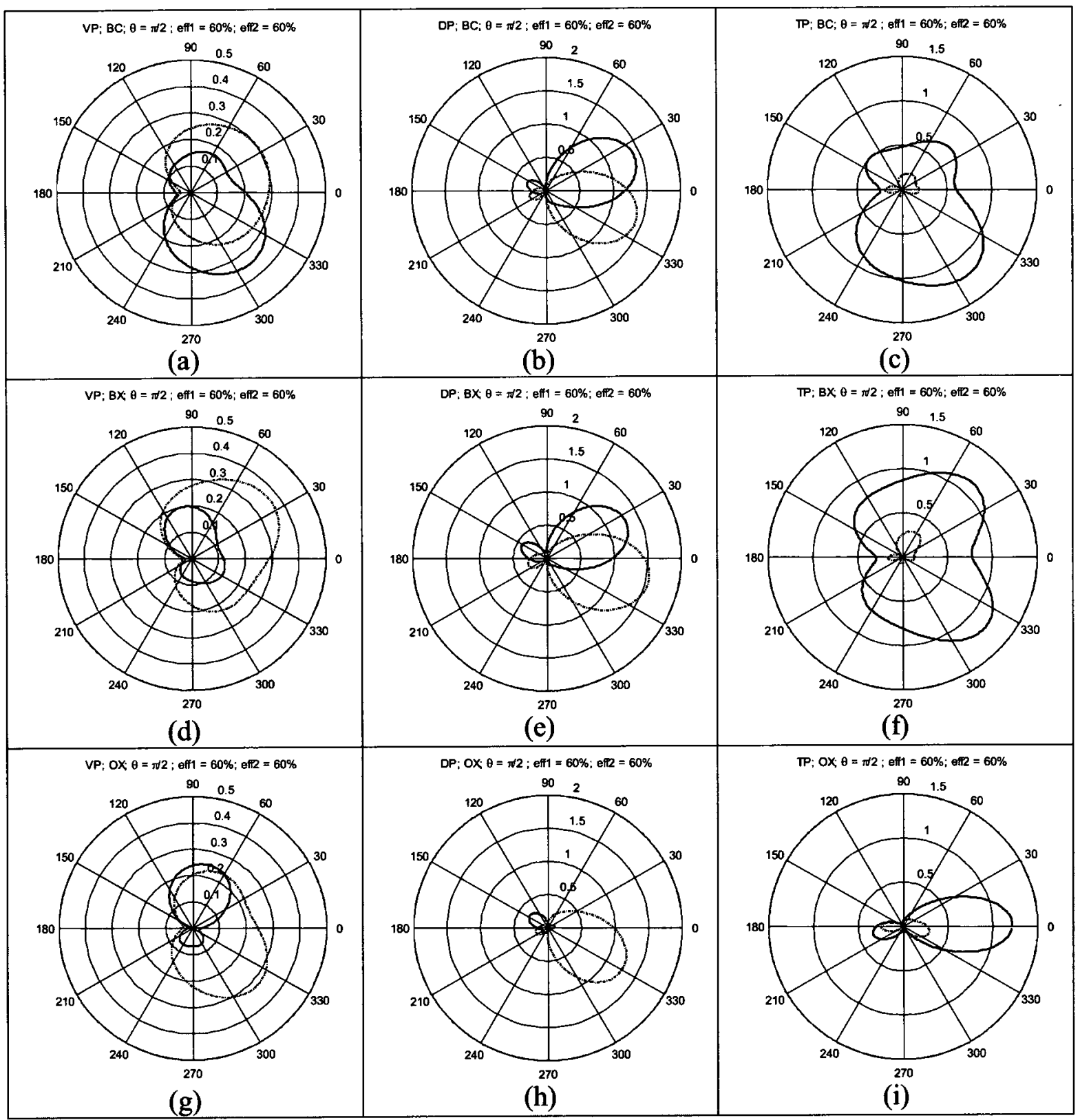

Figure 6.6. Azimuth antenna patterns for the different $R x$ antennas for different configuration/efficiency combinations. The solid line is for Element $\# 1$, and the dashed line is for Element \#2. 


\subsubsection{The Influence of Antenna Configuration, Antenna Efficiency and HT Position}

\section{on Eigenvalue Dispersion}

Recall that (a) $G_{m u x}$ is a measure of eigenvalue dispersion, and (b) when dispersion of the eigenvalues associated with the channel matrix for a MIMO link is high (i.e., their values have a wide range), $G_{m u x}$ is low, and vice versa. A high value of $G_{m u x}$ is desirable on MIMO links, provided SNR is also reasonable, since in this case, the power in all virtual subchannels of the MIMO link is approximately the same. Figures 6.7., 6.8., and 6.9. show ECDFs for $G_{m u x}$ corresponding to various $\mathrm{HT}$ antenna configurations and efficiencies, for the cases when the HT is in the 3 different operating positions.

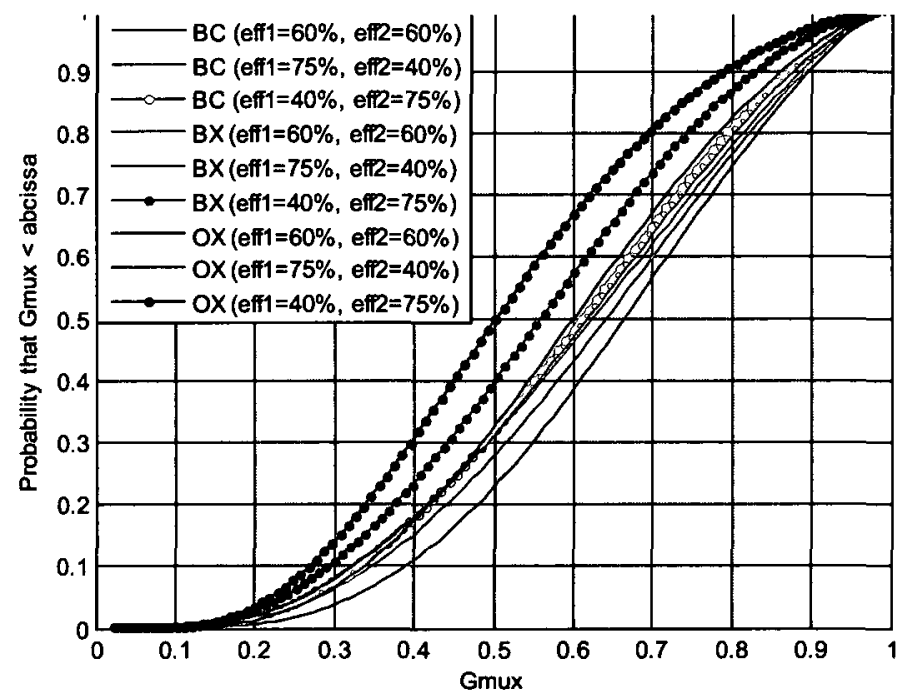

Figure 6.7. CDF for $G_{m u x}$ at the $\mathrm{VP}$ for different configurations and element efficiencies combinations

For the vertical position (Figure 6.7.), it can be observed that $G_{m u x}$ is greatest for the OX configuration, when the "favoured" antenna element is most efficient. When the antenna elements in the $\mathrm{OX}$ configuration are equally efficient, $G_{m u x}$ has values that are lower than the greatest values for the $\mathrm{BC}$ and $\mathrm{BX}$ configurations, but the differences are probably not statistically significant, and the differences between the values for the $\mathrm{BC}$ and $\mathrm{BX}$ configurations are also not considered to be statistically significant, at least for the cases when the HT antenna elements are equally efficient. It should also be noted that, 
although the OX configuration can result in the highest values of $G_{m u x}$, it can also yield the lowest values of $G_{m u x}$ when the "favoured" antenna element is the least efficient.

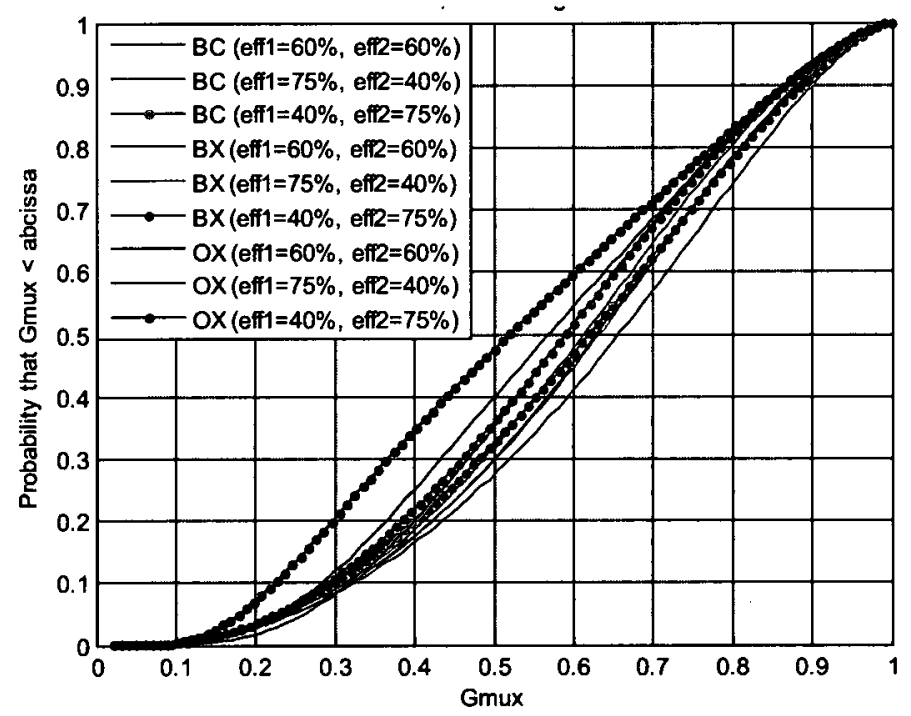

\section{Figure 6.8. CDF for $G_{m u x}$ at the DP for different configurations and element efficiencies combinations.}

In the DP (Figure 6.8.), there is the least disparity among $G_{m u x}$ results. Values for the $\mathrm{BC}$ configuration are the greatest, those for the $\mathrm{BX}$ configuration are slightly lower, though probably not statistically significantly so, and those for the OX case are significantly lower for the poorest efficiency combination and the combination when the two elements are equally efficient, but about the same for the best efficiency combination. 


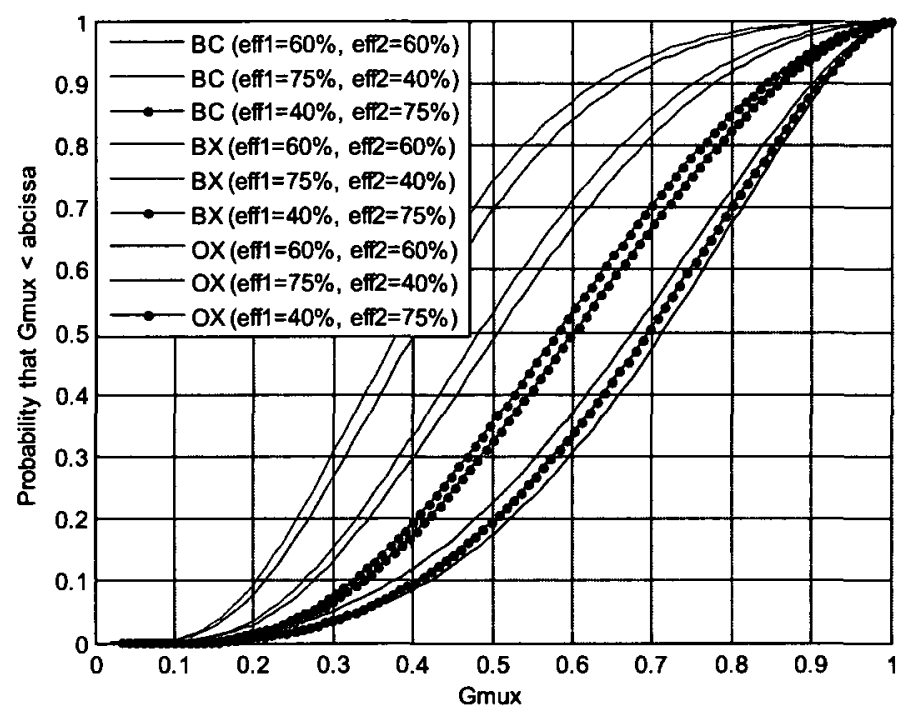

\section{Figure 6.9. CDF for Gmux at the TP for different configurations and element efficiencies combinations.}

In the TP (Figure 6.9.), values for $G_{\max }$ are again greatest for the $\mathrm{OX}$ configuration, and this is so for all efficiency combinations. The disparity among results in this case is the largest. Values for the $\mathrm{BC}$ and $\mathrm{BX}$ configurations track each other closely for each of the three efficiency combinations. These results, for the three efficiency combinations have a very wide disparity.

Based on the foregoing, it seems reasonable to conclude that there is a tradeoff. $\mathrm{G}_{\text {ant, }}$, which directly influences MIMO link SNR at each SL, is always poorest for the OX antenna element configuration, regardless of antenna efficiencies. However, when the HT is in the VP and TP, $G_{\text {mux }}$, which directly influences multiplexing gain, is often greatest (the best) for the OX configuration. It can also be nearly as good, even when its elements are equally efficient, in the DP. Only an evaluation of MIMO capacities, as in the following subsection, can indicate which one of these two characteristics is dominant in establishing achievable capacity limits on the radio links under study. 


\subsubsection{The Influence of Antenna Configuration, Antenna Efficiency and HT Operating}

\section{Position on BS-HT MIMO Link Outage Capacity}

Herein, MIMO link capacity is evaluated in terms of outage capacity, $C_{O}$, at probability $p_{O}$, which is defined through the relationship

$$
P\left[C<C_{o}\right]=p_{o}
$$

Figure. 6.10 shows the relationship between $C_{o}$ and average system SNR, for $p_{o}=0.10$ (i.e., $10 \%$ outage probability) when the HT is in the Vertical operating position.

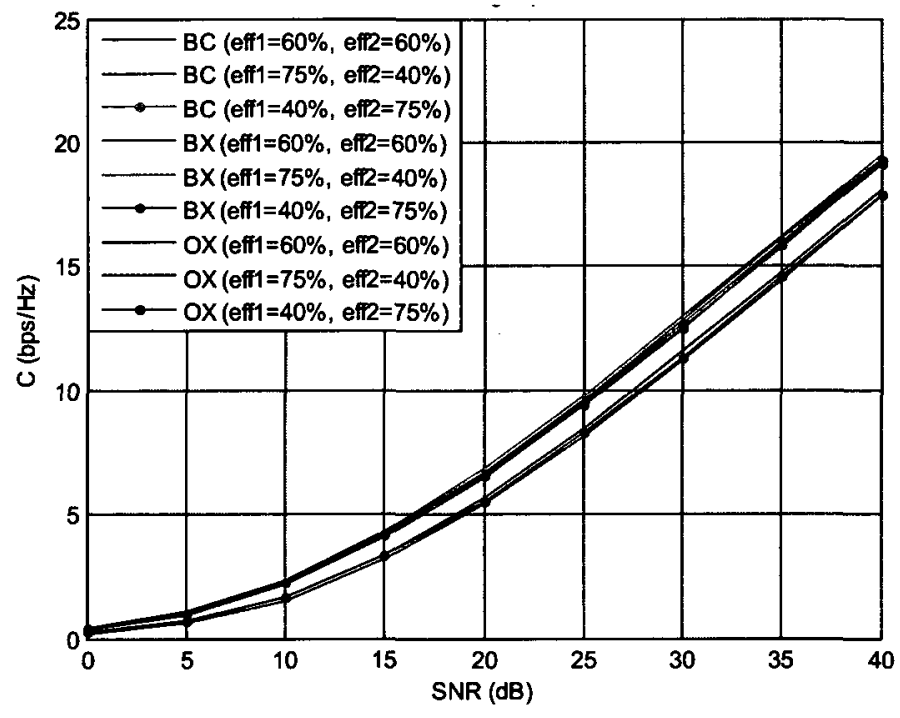

Figure 6.10. Outage capacity $C_{o}$ presented as a function of system SNR, for the case when the HT is in the Vertical operating position. Probability level $p_{o}=10 \%$.

It is clear from Figure. 6.10. that the outage capacities for the $\mathrm{BC}$ and $\mathrm{BX}$ configurations are very close to each other, and that, for all values of average system SNR, they are significantly greater than the outage capacities for the OX configuration. It 
is also clear, that in all cases, the range of outage capacity corresponding to the different combinations of antenna efficiency is less than $1.5 \mathrm{~b} / \mathrm{s} / \mathrm{Hz}$, at all values of SNR. When considered at this level, the matching of HT antenna efficiencies doesn't seem as important as when this was considered based on the examination of ECDFs for $G_{a n t}$. Similar results hold true for each of the other values of $p_{o}$. The same behaviour as that described in previous sentences can be observed when the outage probability is increased, except (a) the disparity among results for different antenna efficiencies becomes greater, but is still not considered significant, and (b) absolute values for outage probability increase.

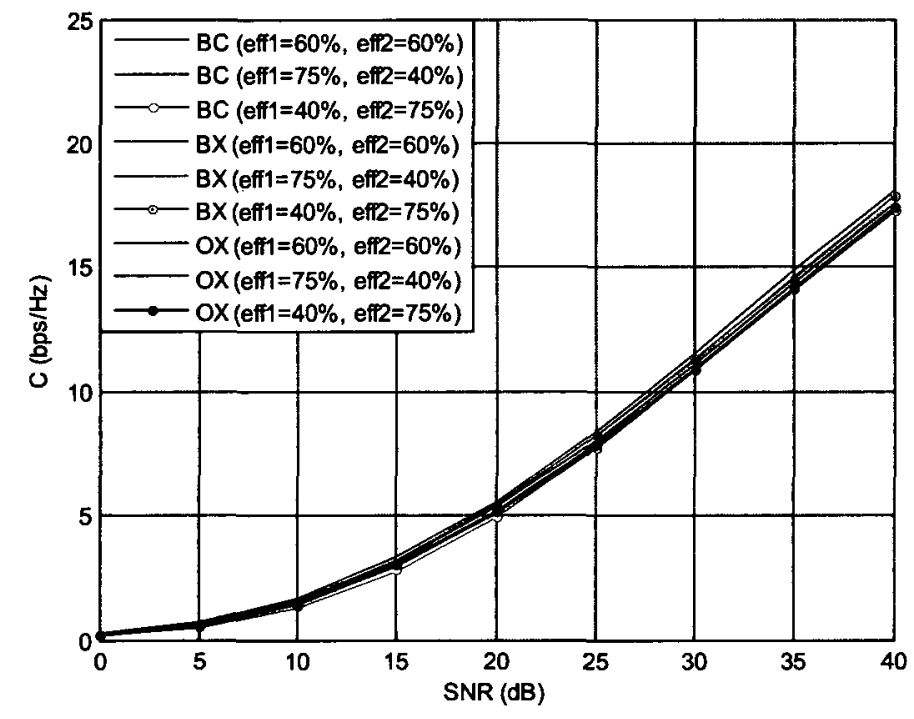

Figure 6.11. Outage capacity $C_{o}$ presented as a function of system SNR for the case when the HT is in the Data operating position. Probability level $p_{o}=10 \%$.

The characteristics reported above for the Vertical HT operating position hold true also for the DP and the TP positions, except that for an outage probability of $10 \%$, results for the BC antenna configuration become slightly lower than those for the OX configuration at lower values of SNR. 
TABLE 6.2. summarizes the results for the BX configuration all $3 \mathrm{HT}$ operating positions, for average system SNRs of $10 \mathrm{~dB}$ and $20 \mathrm{~dB}$.

TABLE 6.2: Outage Capacities of the BX Configuration at Average System SNRs of 10 and $20 \mathrm{~dB}$

\begin{tabular}{|c|c|c|c|c|c|c|}
\hline $\begin{array}{c}\text { Outage } \\
\text { Probability }\end{array}$ & \multicolumn{6}{|c|}{ Outage Capacity (bps/Hz) } \\
\hline-- & Vertical Position & Data Position & \multicolumn{2}{c|}{ Talk Position } \\
\hline Average SNR (dB) & $\mathbf{1 0}$ & $\mathbf{2 0}$ & $\mathbf{1 0}$ & $\mathbf{2 0}$ & $\mathbf{1 0}$ & $\mathbf{2 0}$ \\
\hline $10 \%$ & 2.25 & 6.70 & 1.62 & 5.53 & 2.41 & 6.81 \\
\hline $25 \%$ & 2.79 & 7.49 & 2.08 & 6.49 & 2.92 & 7.64 \\
\hline $50 \%$ & 3.34 & 8.39 & 2.75 & 7.68 & 3.49 & 8.47 \\
\hline $90 \%$ & 4.36 & 10.09 & 4.22 & 9.58 & 4.44 & 9.91 \\
\hline
\end{tabular}

TABLE 6.2. shows that, for all the specified outage probabilities, outage capacity when the best (BX) HT antenna configuration is used only varies by about $0.85 \mathrm{bps} / \mathrm{Hz}$ when the average system SNR is $10 \mathrm{~dB}$ and by about $1.15 \mathrm{bps} / \mathrm{Hz}$ when the average system SNR is $20 \mathrm{~dB}$, for the three HT operating positions that were considered.

\subsubsection{The Influence of Handheld Terminal Orientation on MIMO Link Performance Parameters}

In this section, the influence on MIMO link performance parameters of HT orientation in the azimuth (horizontal) plane is reported. Three types of result are reported, including the ECDF of the average of each performance parameter over $36 \mathrm{HT}$ orientations with 10 degrees of separation between them, the ECDF of the best performance results out of each set for 36 orientations, and the ECDF of the worst performance results out of each set for 36 orientations. 


\subsubsection{The Influence of HT Orientation, Antenna Configuration, Antenna Element Efficiency and HT Operating Position on $\mathrm{G}_{\text {ant }}$}

Figure. 6.12. shows ECDFs of $G_{a n t}$ for the various cases of antenna configuration and efficiency, averaged over 36 different HT orientations, when the HT is in the Vertical operating position.

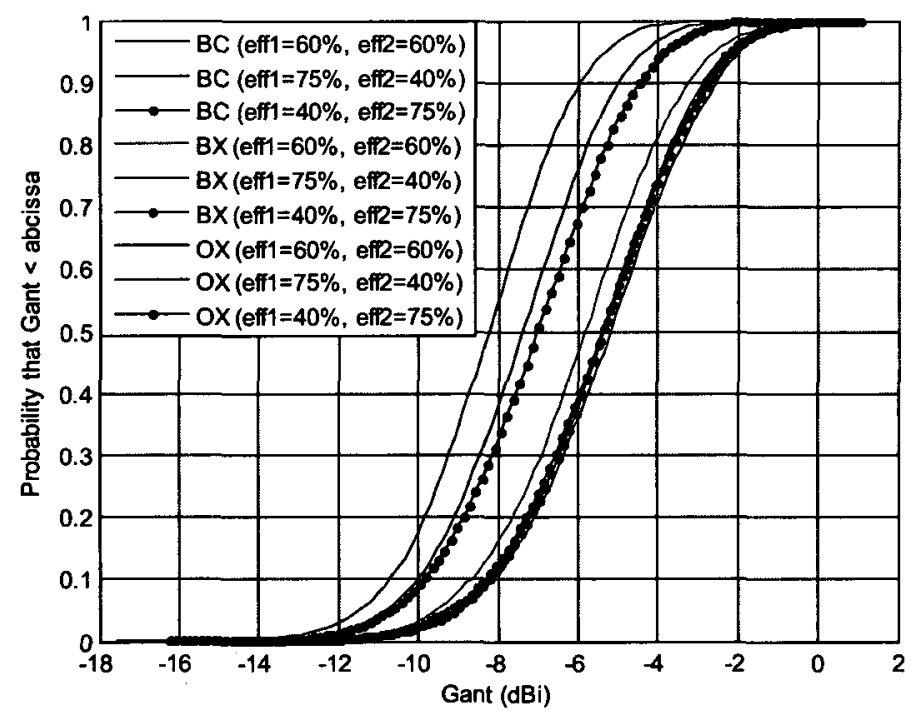

Figure 6.12. ECDFs for the average $G_{a n t}$ over 36 different azimuth orientations spaced by 10 degrees, when the HT is in the Vertical operating position.

The trends shown in Figure 6.12. are very similar to those shown in Figure 6.2., which reports ECDFs of results for all 36 orientations in each SL. The OX antenna configuration results in the lowest values of $\mathrm{G}_{\text {ant }}$, while the $\mathrm{BC}$ and $\mathrm{BX}$ configurations result in the greatest values, probably with no difference of statistical significance between them. At the median, the greatest value of $\mathrm{G}_{\text {ant }}$ is close to $-5.18 \mathrm{dBi}$. This value is approximately the same as the median value for the most effective antenna gains shown in Figure 6.2. 
Figure 6.13. shows the corresponding ECDFs for the greatest values of $\mathrm{G}_{\text {ant }}$ obtained with the HT in the Vertical operating position, presumably obtained with the HT in the "most favourable" of the 36 orientations.

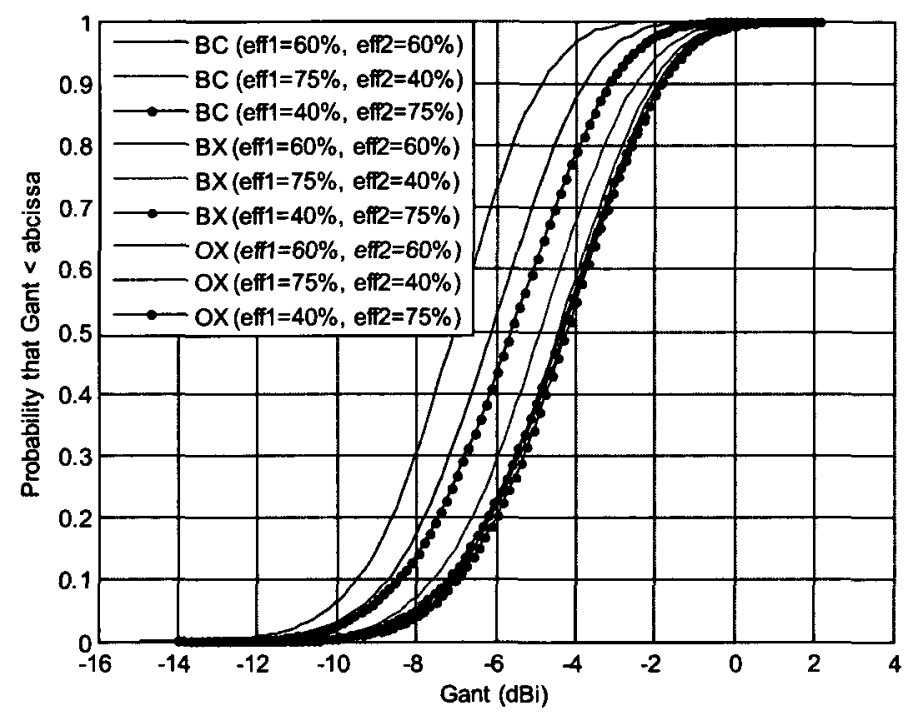

Figure 6.13. ECDFs for the greatest values of $G_{a n t}$ among the 36 values for different orientations of the HT estimated at every SL, when the HT is in the Vertical operating position.

The trends in Figure 6.13. are the same as those in Figures 6.2. and 6.12., but the median of $G_{a n t}$ for the best antenna configuration/efficiency combination is slightly better, being $-4.23 \mathrm{dBi}$, as opposed to $-5.18 \mathrm{dBi}$.

The corresponding ECDFs for the lowest values of $G_{\text {ant }}$, presumably associated with the worst HT orientation are shown in Figure 6.14. 


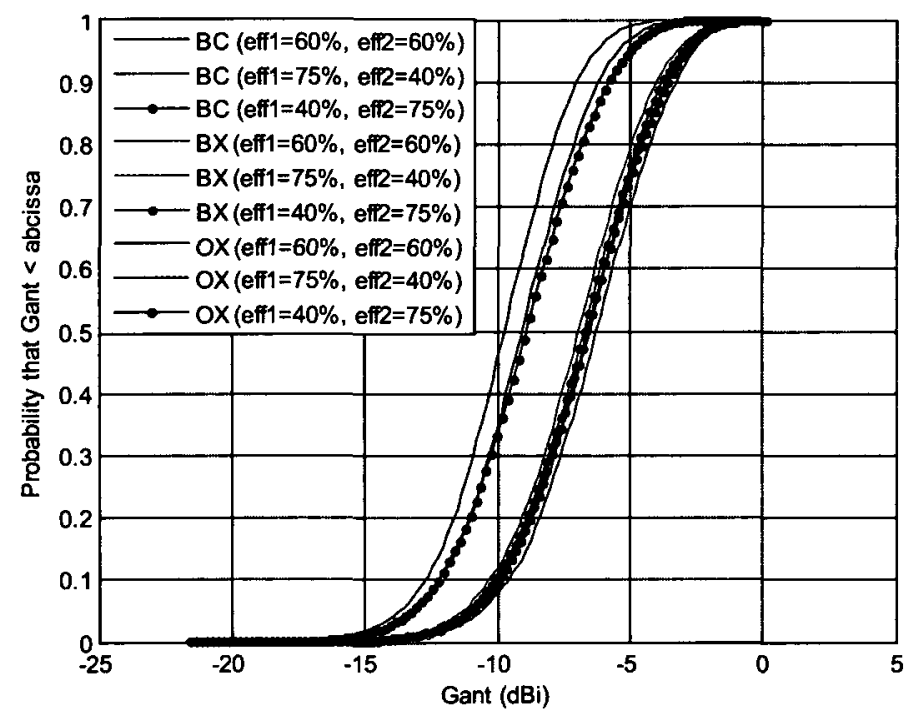

Figure 6.14. ECDFs for the smallest values of $G_{a n t}$ among the 36 values for different orientations of the HT estimated at every SL, when the HF is in the Vertical operating position.

Figure 6.14. shows that the trends in $G_{\text {ant }}$ for different antenna configurations and efficiencies remain the same as for the above-discussed cases. However, the median value of $\mathrm{G}_{\mathrm{ant}}$ for the best (BC) antenna configuration is somewhat lower, being about $-6.27 \mathrm{dBi}$.

For the Data and Talk HT operating positions, trends and relative performance for the different antenna configurations, and efficiencies are much the same as those reported for the corresponding cases in Section 6.5.1. TABLE 6.3. summarizes the median results for the average over all orientations, the best results out of the 36 orientations in each SL, and the worst results of those for the 36 orientations in each SL. 
TABLE 6.3 Medians for the Greatest Values of $G_{\text {ant }}$ in Specified Groups of Results

\begin{tabular}{|l|c|c|c|}
\hline \multicolumn{1}{|c|}{--} & \multicolumn{3}{|c|}{ Gant (dBi) } \\
\hline \multicolumn{1}{|c|}{ Group } & Vertical Position & Data Position & Talk Position \\
\hline $\begin{array}{l}\text { Average of results } \\
\text { for 36 orientations }\end{array}$ & $-5.18^{*}$ & $-7.02^{+}$ & $-3.86^{\#}$ \\
\hline $\begin{array}{l}\text { Best in those for 36 } \\
\text { orientations }\end{array}$ & $-4.23^{*}$ & $-5.61^{+}$ & $-1.95^{\#}$ \\
\hline $\begin{array}{l}\text { Worst in those for } \\
\text { 36 orientations }\end{array}$ & $-6.27^{*}$ & $-8.90^{+}$ & $-6.77^{\#}$ \\
\hline All results & $-5.22^{*}$ & $-7.05^{+}$ & $-3.98^{\#}$ \\
\hline
\end{tabular}

* BC or BX configuration, efficiency combination makes little difference

${ }^{+} \mathrm{BX}$ configuration, efficiency combination makes little difference

${ }^{\#} \mathrm{BX}$ configuration, unbalanced efficiency

TABLE 6.3. indicates that median values for $\mathrm{G}_{\text {ant }}$ are somewhat $(0.5-3.66 \mathrm{~dB})$ greater when the HT is in the Talk operating position. It also shows that the range of median values of $\mathrm{G}_{\mathrm{ant}}$ over all orientations and all three HT positions, for the best (BX) antenna configuration is from $-8.90 \mathrm{dBi}$ (worst) to $-1.95 \mathrm{dBi}$ (best), or about $6.95 \mathrm{~dB}$.

\subsubsection{The Influence of HT Orientation, Antenna Configuration, Antenna Element}

Efficiency and HT Operating Position on Eigenvalue Dispersion.

Figure 6.15. shows ECDFs of $G_{m u x}$ for the various cases of antenna configuration and efficiency averaged over 36 different HT orientations, when the HT is in the Vertical operating position. 


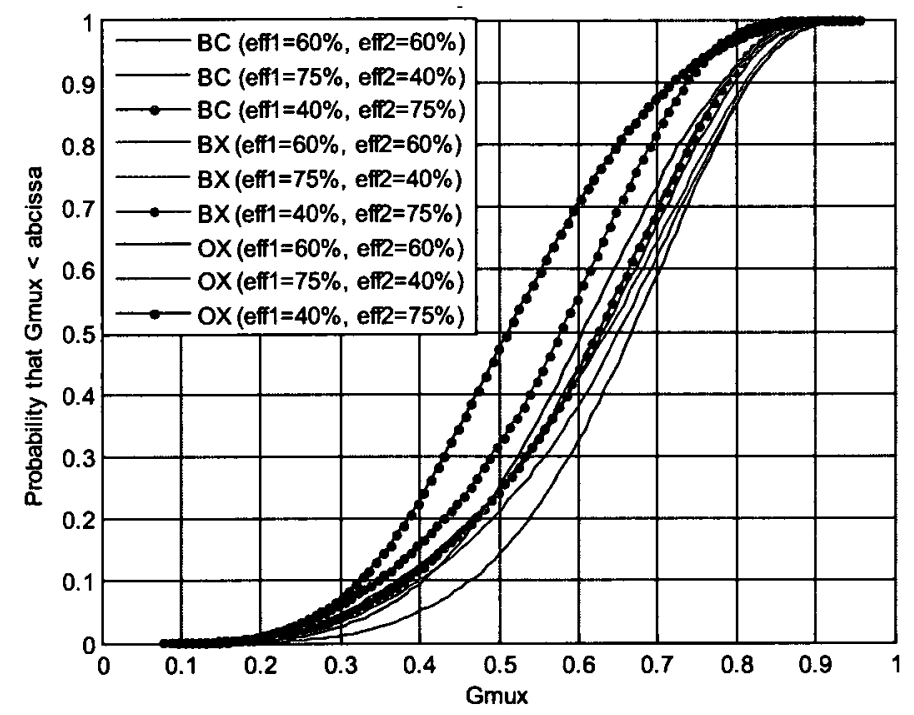

Figure 6.15. ECDFs for the average of $G_{m u x}$ for 36 orientations of the HT in azimuth, for various antenna configurations and efficiencies, when the $\mathrm{HT}$ is in the Vertical operating position.

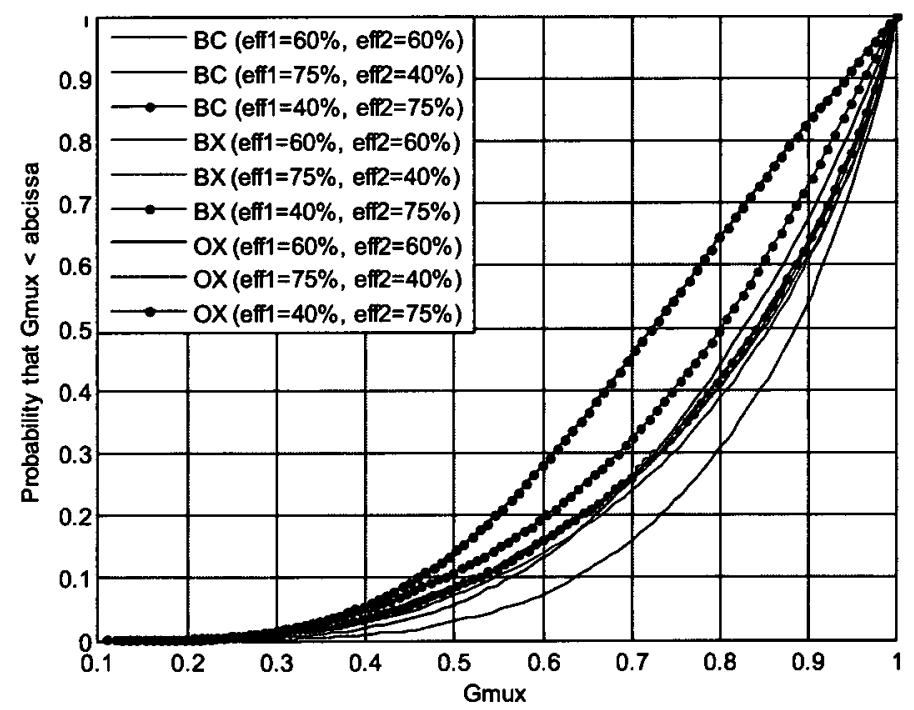

Figure 6.16. ECDFs for the greatest values of $G_{m u x}$ for 36 orientations of the HT in azimuth, for various antenna configurations and efficiencies, when the $\mathrm{HT}$ is in the Vertical operating position. 
Again, results and trends in Figure 6.15. are very similar to corresponding results that can be seen in ECDFs constructed from the total pool of results for all SLs and all orientations. The medians for the best results (in this case for the OX antenna configuration) is about 0.67 .

Figure 6.16. shows corresponding ECDFs for the maxima of the 36 results estimated for each SL. In this case, the trends are about the same (the OX position still yields the greatest dispersion), but the shapes of the ECDFs are distorted, as the normal levelling of the ECDFs at large percentiles is absent. The median of the best (OX) result is about 0.88 .

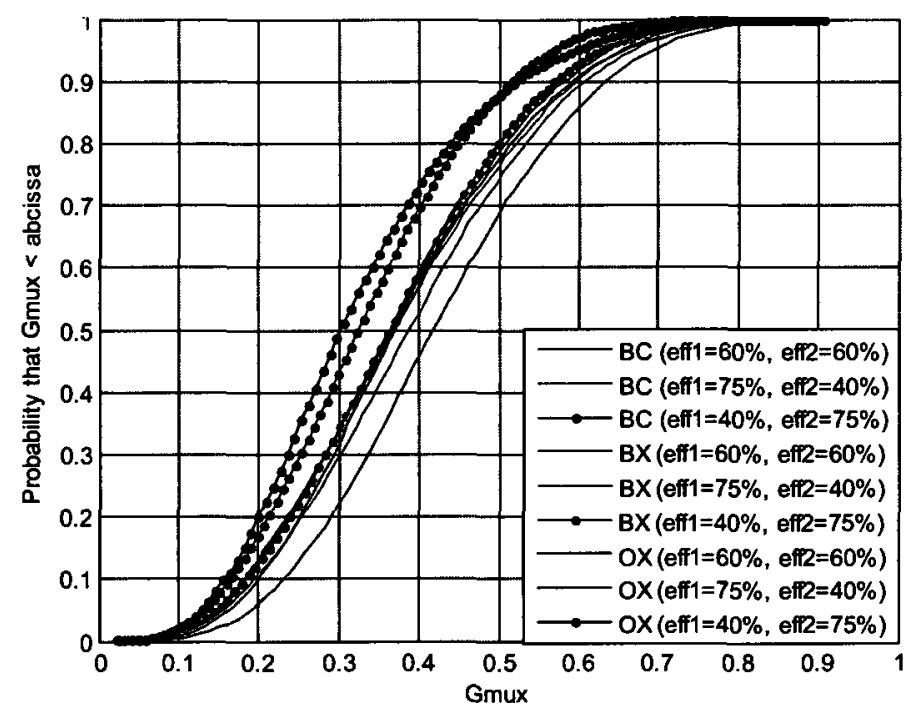

Figure 6.17.ECDFs for the lowest values of $G_{m x}$ for 36 orientations of the HT in azimuth, for various antenna configurations and efficiencies, when the $H T$ is in the Vertical operating position.

The worst $G_{m u x}$ results are shown in Figure 6.17. It can be seen that in this case, the OX position still results in the greatest values of $G_{m u x}$, but the ECDFs are again distorted, this time with their low-probability tails absent. The median value that is the greatest among medians for different antenna configurations and efficiencies is about 0.42 . 
When the HT is in the Data operating position, the greatest values of $G_{m u x}$ are achieved with the BC antenna configuration (balanced efficiencies). When the HT is in the Talk operating position, the greatest values of $G_{m u x}$ are again achieved with the BX configuration (balanced efficiencies). Although the same tail distortions prevail, there is much more disparity in results for different antenna configurations, as well as for different antenna efficiencies, when the HT is in this operating position.

A summary of median results concerning the influence of HT orientation on eigenvalue dispersion can be found in TABLE 6.4.

TABLE 6.4. Medians for the Greatest Values of $G_{m u x}$ in Specified Groups of Results

\begin{tabular}{|c|c|c|c|}
\hline-- & \multicolumn{3}{|c|}{$\boldsymbol{G}_{\text {mux }}$} \\
\hline Group & Vertical Position & Data Position & Talk Position \\
\hline $\begin{array}{c}\text { Average of results } \\
\text { for 36 orientations }\end{array}$ & $.67^{+}$ & $.67^{*}$ & $.71^{\#}$ \\
\hline $\begin{array}{c}\text { Best in those for 36 } \\
\text { orientations }\end{array}$ & $.88^{+}$ & $.91^{*}$ & $.95^{\#}$ \\
\hline $\begin{array}{c}\text { Worst in those for } \\
\text { 36 orientations }\end{array}$ & $.42^{+}$ & $.35^{*}$ & $.38^{\#}$ \\
\hline All Results & $.66^{+}$ & $.65^{*}$ & $.71^{\#}$ \\
\hline
\end{tabular}

${ }^{+} \mathrm{OX}$ configuration, unbalanced efficiencies

*BC configuration, balanced efficiencies

\# OX configuration, balanced efficiencies

TABLE 6.4. shows that the lowest values of eigenvalue dispersion (greatest values of $G_{m u x}$ ) are obtained with different antenna configurations in the three different HT positions. Values in this table also indicate that the lowest values of eigenvalue dispersion are obtained when the HT is in the Talk operating position. TABLE 6.3. indicates that the greatest values of $\mathrm{G}_{\mathrm{ant}}$ are obtained with this same position. One would therefore expect the greatest values of outage capacity to be obtained when the HT is in this position. 


\subsubsection{The Influence of HT Orientation, Antenna Configuration, Antenna Element}

\section{Efficiency and HT Operating Position on Outage Capacity.}

Figure 6.18. shows plots of outage capacity $\left(C_{O}\right)$ at an outage probability of $10 \%$ for the various cases of antenna configuration and efficiency averaged over 36 different HT orientations, when the HT is in the Vertical operating position.

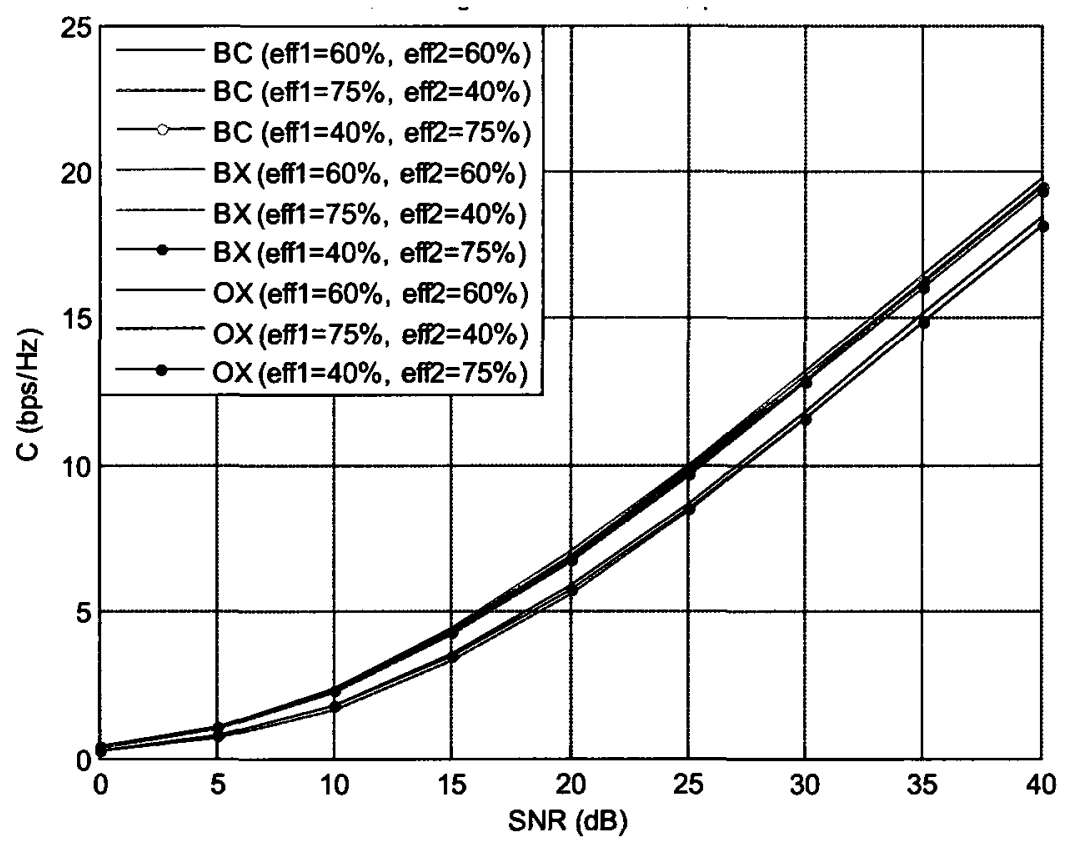

Figure 6.18. Outage capacity at an outage probability of $10 \%$, with the HT in the Vertical operating position.

The figure clearly shows that at all values of average system SNR, capacity is greatest when the antenna elements are at the bottom of the terminal. This is the same as the result obtained without the averaging over all orientations before estimation of the ECDF. Outage capacity is significantly lower for the OX antenna configurations regardless of the efficiency combination. The same behaviour is true at all outage probabilities and for the averages over 36 orientations in azimuth, as well as for the best and worst cases. 
Results for outage capacities as a function of HT orientation in azimuth with the terminal in the Data operating position show that the highest outage capacities are always achieved with the BX antenna configuration. Results for different antenna efficiencies are not distinguishable. In this case, there may sometimes be a statistically significant difference between $\mathrm{BX}$ and $\mathrm{BC}$ results. OX results are sometimes as good as $\mathrm{BC}$ results, depending on outage probability.

With the HT in the Talk operating position, results are again consistently the best with the $\mathrm{BX}$ antenna configuration. At some values of outage probability $\mathrm{BC}$ and $\mathrm{OX}$ results are almost as good. However, at other outage probabilities there is a significant disparity among results.

A summary of Median capacity results for selected outage probabilities can be seen in TABLE 6.5.

TABLE 6.5. Outage Capacities for an Average System SNR of $10 \mathrm{~dB}$ for Specified Groups of Results

\begin{tabular}{|c|c|c|c|c|c|c|c|c|c|c|c|c|}
\hline & \multicolumn{10}{|c|}{ Outage Capacity (bps/Hz) } \\
\hline & \multicolumn{1}{|c|}{ Vertical Position } & \multicolumn{3}{c|}{ Data Position } & \multicolumn{4}{c|}{ Talk Position } \\
\hline Outage Prob. (\%) & $\mathbf{1 0}$ & $\mathbf{2 5}$ & $\mathbf{5 0}$ & $\mathbf{9 0}$ & $\mathbf{1 0}$ & $\mathbf{2 5}$ & $\mathbf{5 0}$ & $\mathbf{9 0}$ & $\mathbf{1 0}$ & $\mathbf{2 5}$ & $\mathbf{5 0}$ & $\mathbf{9 0}$ \\
\hline $\begin{array}{c}\text { Average of results for } \\
\text { 36 orientations }\end{array}$ & 2.40 & 2.85 & 3.42 & 4.50 & 1.71 & 2.13 & 2.72 & 4.14 & 2.70 & 3.00 & 3.54 & 4.38 \\
\hline $\begin{array}{c}\text { Best in those for 36 } \\
\text { orientations }\end{array}$ & 2.76 & 3.30 & 3.90 & 5.00 & 2.10 & 2.64 & 3.35 & 4.79 & 3.35 & 3.77 & 4.23 & 5.07 \\
\hline $\begin{array}{c}\text { Worst in those for 36 } \\
\text { orientations }\end{array}$ & 1.93 & 2.35 & 2.93 & 3.87 & 1.15 & 1.55 & 2.10 & 3.58 & 1.73 & 2.16 & 2.68 & 3.72 \\
\hline All Results & 2.32 & 2.86 & 3.45 & 4.58 & 1.62 & 2.08 & 2.75 & 4.25 & 2.47 & 3.03 & 3.53 & 4.53 \\
\hline
\end{tabular}

The values in TABLE 6.5 . indicate that median outage capacities can vary by 1 $\mathrm{bps} / \mathrm{Hz}$ as a function of HT orientation, for the Vertical HT operating position. For the data and Talk operating positions, these medians vary by about $1.25 \mathrm{bps} / \mathrm{Hz}$ and 1.55 $\mathrm{bps} / \mathrm{Hz}$ with HT orientation, respectively. The table also shows that the largest outage capacities can be obtained with the HT in the Talk operating position. The lowest capacities are obtained with the HT in the Data operating position. 


\subsection{Summary}

- The greatest values of $G_{a n t}$ can be obtained when the antennas are in the bottom of the HT, regardless of its position. The statistical difference of those results for the cases of co-polarized or cross-polarized bottom elements is considered negligible most of the time. In a few instances $G_{a n t}$ is greater when these antennas are cross-polarized. $G_{a n t}$ is significantly poorer in most instances when the opposite, cross-polarized antenna pair is used.

- $G_{a n t}$ is often greatest when the antennas are not equally efficient. However, it is considered that this is because the unbalanced cases studied in this work all had one antenna that was more efficient than the antennas studied in the balanced cases. Even under these conditions, values of $G_{a n t}$ are not significantly lower when the antennas were equally efficient. This leads to the conclusion that two equally efficient antennas would give the best performance, provided that they are highly efficient.

- The smallest values of eigenvalue dispersion can be obtained when the HT antennas are on opposite sides of the terminal, with one at the top and one at the bottom, and when these antennas are cross-polarized (the so-called OX configuration). When the HT is in the Talk operating position, the difference with respect to eigenvalue dispersion with the other antenna configurations can be significant.

- The greatest outage capacities can be obtained when the antennas are at the bottom of the HT, and are cross-polarized. When one considers the foregoing conclusion, which indicates that diversity and multiplexing gains would be greater much of the time when another $(\mathrm{OX})$ antenna configuration were employed, it is clear that average MIMO system SNR, which is influenced directly by $G_{a n t}$, is more valuable over eigenvalue dispersion in determining MIMO link capacity. 
- Statistical distributions for $G_{a n t}$, eigenvalue dispersion and outage capacity appear to be the same whether results for a single street location are averaged over orientation before the distributions are estimated or not.

- When there is perfect power control in the system under study, and the SNR on every base station to handheld terminal link is $10 \mathrm{~dB}$, the difference between the worst achievable and best achievable capacity at the medians of the distributions estimated using data from all street locations is never greater than $1.55 \mathrm{bps} / \mathrm{Hz}$, for any of the three HT positions that were considered, at any of the outage probabilities that were considered.

- Under conditions of perfect power control in the system under study, when results from all street locations are considered without any averaging before statistical distributions are estimated, outage capacities between 1.62 and $2.47 \mathrm{bps} / \mathrm{Hz}$, between 2.08 and $3.03 \mathrm{bps} / \mathrm{Hz}$, between 2.75 and $3.53 \mathrm{bps} / \mathrm{Hz}$, and between 4.25 and $4.58 \mathrm{bps} / \mathrm{Hz}$ can be achieved regardless of which of the three HT operating positions considered herein is employed, when the average MIMO link SNR is 10 $\mathrm{dB}$, at outage probabilities of $10,25,50$, and 90 percent, respectively. These values are for a $4 \times 2$ MIMO microcellular system having a BS with an antenna height of around $6 \mathrm{~m}$ above street level in a high-rise environment, when there is no line of sight between the BS and the HT, and when multipath signals that are received at the HT depart from the BS with a uniform distribution of angles in the azimuth plane. The stipulation of a uniform probability distribution for AODs at the BS is an assumption that was made during the work reported here, in the absence of experimental data. It is considered that this distribution may, in fact, apply, under the NLOS conditions that were studied. However, this is not known with certainty. It is considered that the effect of an error in this assumption on the comparisons reported herein among link performance parameters for the different antenna configurations and HT positions would be insignificant. However, the effect of such an error on the actual (rather than relative) values of these parameters would probably be to decrease eigenvalue dispersion, and yield outage capacity estimates that are higher than those that can actually be achieved. 


\section{CHAPTER 7}

\section{SUMMARY, CONCLUSION AND TOPICS FOR FURTHER STUDY}

\subsection{Summary}

The objective of the thesis project reported herein was about characterizing and utilizing multipath clusters on radio channels and the subsequent prediction of the performance of multi-antenna mobile radio systems. Based on propagation measurement data, analysis of the clustering nature of wireless channels was reported in Chapter 4, where the identification of multipath clusters was used to aid in modelling the timevarying behaviour of the measured channels. The following are reported: a new algorithm for the identification of multipath clusters, a new method to find the length of intervals, referred to herein as consistency intervals, in which multipath characteristics remain fairly constant, and a new algorithm for modelling variations, with time, of the power in multipath clusters.

In the work reported in Chapter 5, the knowledge of the clustering nature of wireless channels was taken advantage of in order to develop new transmission techniques that improve multi-antenna system performance in MIMO broadcasting systems and cognitive radio systems. A new algorithm that performs joint Tx-Rx beamforming toward interacting objects associated with the strongest clusters was introduced. The algorithm that was introduced increases the multiuser diversity in MIMO broadcasting systems. In addition, this algorithm was modified and further applied to allow the simultaneous coexistence of primary users, as well as secondary users in cognitive radio systems, in the same frequency band, without violating the QoS at the terminals of the primary users.

In Chapter 6, propagation measurement data was used to evaluate the performance of MIMO radio links in which user handheld terminals have a number of alternate receive antennas. MIMO channel matrices synthesized using multipath parameter estimates from the propagation data were used to study the effect of the antenna configuration, handheld 
terminal operating position, and the efficiency of antenna elements on MIMO radio link performance.

\subsection{Conclusion}

The work reported in this thesis gives significant insight into the spatial structure of real-world multiple antenna channels and its effect on the ability of MIMO systems in constructing multiple spatial layers of data streams. A useful picture of the influence of the spatial structure of MIMO channels can be obtained by thinking of the arrival of the received radio signals as the reception of number of streams of energy that arrive through multiple different pipes. It is concluded that not only do such "effective pipes" exist, but the numbers of these pipes and their parameters have a time-varying nature. This work also has shown that the characterization of this continuously-changing "flow" of energy through multipath clusters involves a number of challenges, with many questions yet to be fully answered.

The first challenge is to correctly identify legitimate multipath clusters. To avoid the time-consuming visual inspection approach, several automatic clustering techniques have previously been reported. Some of these so-called "clustering" techniques are variations of the vector quantization (VQ) technique, which is often used successfully in applications where different dimensions of the space of interest have the same units. For example, in image processing, both the $\mathrm{x}$-axis and y-axis have the same units: pixels. Applying such VQ-based technique to identify multipath clusters leads to the problem of having to deal with parameters whose dimensions are different in nature, such as delay and AOA. Using parameter normalization or the Kernel density estimation techniques to solve this problem yields results that are, intuitively, not completely satisfactory.

An alternate approach that has previously been used to identify multipath clusters depends on performing the clustering sequentially in different dimensions. While this approach avoids the complexity and intuitive concerns resulting from dealing jointly with parameters having different natures, it neglects consideration of the interdependent character of delay and angle parameters, which results naturally from the geometrical structure of interacting objects in environments surrounding a radio link. Consideration of these facts led to the conclusion that pre-existing clustering methods had to be improved 
upon. It is believed the needed improvements have been provided during the work reported here by the introduction of a new clustering algorithm. This new algorithm avoids normalizing parameters with different natures, and takes the joint distribution of delay and angle parameters into consideration. In doing so, it maintains the "sense" of the visual meaning of multipath clusters, since the shapes of the identified clusters are almost identical to those of the clusters that would have resulted from using the visual inspection method. These shapes reflect the influence of the geometrical structure of the interacting objects on the delay and angular parameters of each cluster. The proposed algorithm can be used to successfully identify both active intervals for the different SSMCs, and dormant intervals. However, the proposed algorithm fails to identify the cases where an AMC region contains two or more SSMCs (i.e., it fails to detect when a multipath cluster splits into two or more clusters). Implementations of such ability must be left as a topic for further study.

A second challenge is associated with characterizing the time-varying nature of multipath clusters. In the work reported in this thesis, the number of multipath clusters and the change in their power, rms DS, and rms AS, were modelled. However, no attempt was made to model the change in the location of a particular multipath cluster in the delay and angular domains. In addition, the statistics of the consistency intervals were defined by assuming thresholds with different arbitrary values. When using such statistics to model the length of the simulated "drops" or "segments," it is believed that the specific "true and meaningful" threshold value should be decided on in accordance with the resolution of the simulated parameters. For example, it is not meaningful to simulate consistency intervals according to statistics coming from wideband measurements if the simulator itself is narrowband. Finding the different statistics of consistency intervals that can be used in simulators with different resolutions in the delay and angular domains is not addressed in this work. It is concluded, however, that this is a necessary extension of this work.

Despite all the modelling complications that are caused by the time-varying nature of multipath clusters, a significant part of the work in this thesis targeted the utilization of such clusters to improve the performance of multiple antenna systems. It is concluded 
from this work that the concentration of a significant portion of the power of radio signals towards IOs that give rise to a few multipath clusters leads to significant benefit from the use of multipath clusters as "paths" or "pipes" in the transfer of signal energy from a BS $T x$ to the user's receivers in multiuser systems. The usefulness of this principle was herein demonstrated for both broadcasting and cognitive radio systems. Based on this successful demonstration, it can also be concluded that the availability of an accurate model that incorporates the time-varying nature of multiple antenna channels would give more accuracy to the performance of the introduced beamforming-based transmission techniques. It is further believed that the slower changes in the power, delay, and angular parameters of multipath clusters could allow time for learning that could result in (a) reducing the number of needed feedback bits and (b) increasing the effectiveness of the proposed beamforming-based techniques; however, if not properly tracked, these slow changes could also negatively affect the multiuser diversity gain achieved by those techniques. Unfortunately, the modelling of time variations on the channels studied was performed subsequent to the investigation of benefits from incorporating consideration of clustering into broadcasting and cognitive system designs. The extension of such system designs so as to be adaptive to changing channel characteristics had, therefore, to be left as a topic for further study.

When evaluating MIMO wireless channels, the focus is usually given to the capacity gain that can be achieved via spatial multiplexing. However, it is also concluded that the initial claims of a few others that a full evaluation of a MIMO link should not only consider its spatial characteristics, but also variations in power gain that influence local SNR, are valid. The work reported in Chapter 6 demonstrated that different antenna parameters such as radiation pattern, position, and orientation affect both the multiplexing gain and the effective power gain of a MIMO link. The compromise between the achievements of the best of these two was studied. This work revealed that, on average, the effective power gain of a MIMO link has greater effect on the achievable capacity than multiplexing gain. It was also concluded that when there is no control over the orientation of the receive device, the closer the radiation patterns of the antenna elements are to isotropic, the higher the achieved capacity. This is because collecting the power of 
the received signal from all directions has more effect in increasing the achievable capacity than the effect of increasing the multiplexing gain.

\subsection{Topics for Further Study}

In the near future, CRC plans to make double-directional sounding measurements from which the AOD (at sounder Tx) of each MPC can be estimated, in addition to all the parameters that were estimated from the measurements reported in this thesis. Based on such double-directional measurements, the work of this thesis could be extended as follows:

The 2D clustering algorithm (introduced in Chapter 4) could be extended to group the MPCs in 3D (i.e., AOD, AOA, and delay). This could be done by extracting the 1D waveform using a vector that scans the $3 \mathrm{D}$ space, and then applying the $1 \mathrm{D}$ valley detection algorithm to each of the resulting 1D waveforms. Following the same steps used in Chapter 4, the parameters of the multipath clusters and their time-varying behaviour could be modelled. The resulting models could be used to build a software channel simulator. However, the proposed algorithm fails to detect when a multipath cluster splits into two or more clusters. Implementation of such an ability is left as a topic for further study.

Finding the different statistics of consistency intervals that can be used in simulators with different resolutions in the delay and angular domains is not addressed in this work. It is believed however, that this is a necessary extension of this work. The results from modelling the lengths of consistency intervals and the time-varying behaviour of multipath clusters can be integrated within existing channel models, for example, to identify the length of the simulation "drops."

The performance comparison work performed in Chapter 6 could employ doubledirectional results (instead of using the uniform AOD assumption) to do further evaluation for different $\mathrm{Rx}$ antennas. The results from using the double-directional measurements could also be compared with the results reported in Chapter 6 to study the effect of the uniform AODs assumption on the capacities of MIMO-links. 
The work in Chapter 5 could be extended to use wireless channel multipath clusters (using the AOD estimation approach and the joint $\mathrm{Tx}-\mathrm{Rx}$ beamforming) within a cross-layer frame work to improve system performance in cooperative communication systems. Multipath clusters on wireless channels can be visualized as being a rich and massive network of "pipes" (with different sizes) connecting the communication terminals where, cooperatively, the proposed techniques could be used to determine the "best pipes" (i.e., the best clusters) to be used such that system performance is optimized.

The results from Chapter 4 show that the strong clusters on wireless channels stay intact for a reasonably long interval of time. This fact leads to the idea of having communication terminals use the AOD estimation/ joint $\mathrm{Tx}-\mathrm{Rx}$ beamforming approach to learn about their environment (i.e., the surrounding strong clusters) in a gradual manner and adapt their transmission/receiving techniques (i.e., the joint $\mathrm{Tx}-\mathrm{Rx}$ beamforming) accordingly. It is believed that this gradual learning/adaptation would have a strong effect on reducing the needed feedback bits, improving the accuracy of estimating the quality and directionality of the channel, and accordingly, improving the joint $T x-R x$ beamforming. 


\section{REFRENCES}

[1] M. Steinbauer, A. Molisch and E. Bonek, "The double-directional radio channel," IEEE Antennas and Propagation Magazine, vol. 43, no. 4, pp. 51-63, Aug. 2001.

[2] P. Almers, et al. "Survey of channel and radio propagation models for wireless MIMO systems," EURASIP Journal on Wireless Communications. and Networking, ID 19070, 2007.

[3] G. El-Zein, R. Cosquer, J. Guillet, H. Farhat and F. Sagnard, "Characterization and modeling of the MIMO propagation channel: an overview," The European Conference on Wireless Technology, pp. 11-14, Oct. 2005.

[4] K. Yu, M. Bengtsson, B. Ottersten, D. McNamara, P. Karlsson and M. Beach, "Second order statistics of NLOS indoor MIMO channels based on $5.2 \mathrm{GHz}$ measurements," IEEE Global Telecommunications Conference, vol. 1. pp. 156160, San Antonio, TX, 25-29 Nov. 2001.

[5] R. Ertel, P. Cardieri, K. Sowerby, T. Rappaport and H. Reed "Overview of spatial channel models for antenna array communication systems " IEEE Personal Communications, vol. 5, no. 1, pp. 10- 22, Feb. 1998.

[6] M. Jensen and J. Wallace, "A review of antennas and propagation for MIMO wireless communications," IEEE Transactions on Antennas and Propagation, vol. 52, no. 11, pp. 2810-2824, Nov. 2004.

[7] J. Kermoal, L. Schumacher, K. Pedersen, P. Mogensen and F. Frederiksen, "A stochastic MIMO radio channel model with experimental validation" IEEE Journal on Selected Areas in Communications, vol. 20, no. 6, pp. 1211-1226, Aug. 2002.

[8] J. Wallace and M. Jensen, "Modeling the indoor MIMO wireless channel," IEEE Transactions on Antennas and Propagation, vol. 50, no. 5, pp. 591-599, May 2002. 
[9] K. Pedersen, J. Andersen, J. Kermoal, and P. Mogensen, “A stochastic multipleinput multiple-output radio channel model for evaluation of space-time coding algorithms," IEEE Vehicular Technology Conference, pp. 893-897, Boston, MA, Sept. 2000.

[10] C. Chuah, J. Kahn, and D. Tse, "Capacity of multi-antenna array systems in indoor wireless environment," IEEE Global Communications Conference, vol. 4, pp. 1894-1899, Sydney, Australia, 1998.

[11] C. Oestges, "Validity of the Kronecker model for MIMO correlated channels," IEEE 63rd Vehicular Technology Conference, vol. 6, pp. 2818-2822, Spring 2006.

[12] H. Ozcelik, M. Herdin, W. Weichselberger, J. Wallace and E. Bonek, "Deficiencies of 'Kronecker' MIMO radio channel model," Electronics Letters, vol. 39, no. 16, pp. 1209-1210, Aug. 2003.

[13] Y. Yang, G. Xu and H. Ling, "An experimental investigation of wideband MIMO channel characteristics based on outdoor Non-LOS measurements at $1.8 \mathrm{GHz}$," IEEE Transactions on Antennas and Propagation, vol. 54, no. 11, part 1, pp. 3274-3284, Nov. 2006.

[14] W. Weichselberger, M. Herdin, H. Ozcelik and E. Bonek, "A stochastic MIMO channel model with joint correlation of both link ends," IEEE Transactions on Wireless Communications, vol. 5, no. 1, pp.90-100, Jan. 2006.

[15] W. Weichselberger, "Spatial structure of multiple antenna radio channel - a signal processing viewpoint," Ph.D. Thesis, Technischen Universität Wien, Fakultät für Elektrotechnik und Informationstechnik, Dec. 2003.

[16] P. Petrus, J. Reed, and T. Rappaport, "Geometrical-based statistical macrocell channel model for mobile environments," IEEE Transactions on Communications, vol. 50, no. 3, pp. 495-502, Mar. 2002. 
[17] C. Oestges, V. Erceg and A. Paulraj, "A physical scattering model for MIMO macrocellular broadband wireless channels," IEEE. Journal on Selected Areas in Communications, vol. 21, no. 5, pp. 721-729, June 2003.

[18] V. Kolmonen, J. Kivinen, L. Vuokko and P. Vainikainen, "5.3-GHz MIMO radio channel sounder," IEEE Transactions on Instrumentation and Measurement, vol. 55, no. 4, pp. 1263-1269, Aug. 2006.

[19] S. Wyne, A. Molisch, P. Almers, G. Eriksson, J. Karedal and F. Tufvesson, "Statistical evaluation of outdoor-to-indoor office MIMO measurements at 5.2 GHz," IEEE 61st Vehicular Technology Conference, vol. 1, pp. 146-150, 30 May-1 June 2005.

[20] L. Smoczynski, A. Gallo and M. Marciniak, "COST action 273 at the National Institute of Telecommunications,", IEEE 5th International Conference on Transparent Optical Networks, vol. 2, pp 137-140, 29 June-3 July 2003.

[21] L. Correia, "COST - 273 towards mobile broadband multimedia networks," IEEE 12th International Conference on Antennas and Propagation, vol. 2, pp. 569-572, 31 Mar.-3 Apr. 2003.

[22] D. Baum, J. Hansen and J. Salo, "An interim channel model for beyond-3G systems: extending the 3GPP spatial channel model (SCM)," IEEE 61st Vehicular Technology Conference, vol. 5, pp.3132-3136, 30 May-1 June 2005.

[23] S. Daniel et al, "Final report on link level and system level channel models," D5.4 v. 1.4, IST-2003-507581 WINNER, Nov. 18th, 2005.

[24] K. Pekka et al, "WINNER II interim channel models," D1.1.1 V1.1, IST-4-027756 WINNER II, 09/02/2007.

[25] M. Narandzic, C. Schneider, R. Thoma, T. Jamsa, P. Kyosti and Z. Xiongwen, "Comparison of SCM, SCME, and WINNER channel models," IEEE 65th Vehicular Technology Conference, pp. 413-417, 22-25 April 2007.

[26] A. Molisch, H. Asplund, R. Heddergott, M. Steinbauer and T. Zwick, "The COST259 directional channel models part I: overview and methodology," IEEE 
Transactions on Wireless Communications, vol. 5, no. 12, pp. 3421-3433, Dec. 2006.

[27] H. El-Sallabi, D. Baum, P. Zetterberg, P. Kyosti, T. Rautiainen and C. Schneider, "Wideband spatial channel model for MIMO systems at $5 \mathrm{GHz}$ in indoor and outdoor environments," IEEE 63rd Vehicular Technology Conference, vol. 6, pp. 2916-2921, Spring 2006.

[28] R. Bultitude, G. Brussaard, M. Herben, and T. Willink, "Radio channel modelling for terrestrial vehicular mobile applications," Millennium Conference in Antennas and Propagation, Davos, Switzerland, April 2000.

[29] I. Viering, H. Hofstetter and W. Utschick, "Validity of spatial covariance matrices over time and frequency," IEEE Global Communications Conference, pp. 851855, Nov. 2002.

[30] J. Wallace, M. Jensen, A. Gummalla and H. Lee, "Experimental characterization of the outdoor MIMO wireless channel temporal variation," IEEE Transactions on Vehicular Technology, vol. 56, no. 3, pp. 1041-1049, May 2007.

[31] M. Herdin and E. Bonek, "A MIMO correlation matrix based metric far characterizing non-stationarity", in The IST Mobile and Wireless Communications Summit, Lyon, 2004.

[32] M. Herdin, "Non-stationary indoor MIMO radio channels," Ph.D. dissertation, Vienna University of Technology, Vienna, Austria, 2004.

[33] M. Herdin, N. Czink, H. Ozcelik and E. Bonek, "Correlation matrix distance, a meaningful measure for evaluation of non-stationary MIMO channels," IEEE 61st Vehicular Technology Conference, vol. 1, pp. 136-140, 30 May-1 June 2005.

[34] R. Bultitude, T. Willink, M. Herben, and G. Brussaard, "Detection of changes in the spectral characteristics of measured mobile radio data for space wave modeling applications," Queen's University Biennial Symposium Communications, pp. 90-94, Kingston, Canada, May 2000. 
[35] M. Toeltsch, J. Laurila, K. Kalliola, A. Molisch, P. Vainikainen and E. Bonek, "Statistical characterization of urban spatial radio channels," IEEE Journal on Selected Areas in Communications, vol. 20, no. 3, pp.539-549, April 2002.

[36] T. Zwick, C. Fischer and W. Wiesbeck, "A stochastic multipath channel model including path directions for indoor environments," IEEE Journal on Selected Areas in Communications, vol. 20, no. 6, pp. 1178-1192, Aug. 2002.

[37] C. Chong and C. Tan, "A new statistical wideband spatio-temporal channel model for 5-GHz band WLAN systems," IEEE Journal on Selected Areas in Communications, vol. 21, no. 2, pp. 139-150, Feb. 2003.

[38] H. Asplund, A. Glazunov, A. Molisch, K. Pedersen, and M. Steinbauer, "The COST 259 directional channel model part II: macrocells," IEEE Transactions on Wireless Communications, vol. 5, no. 12, pp. 3434-3450, Dec. 2006.

[39] L. Vuokko, P. Vainikainen, and J. Takada, "Clusters extracted from measured propagation channels in macrocellular environments, "IEEE Transaction on Antennas and Propagation, vol. 53, no. 12, pp. 4089-4098, Dec. 2005.

[40] N. Czink, P. Cera, J. Salo, E. Bonek, J.P. Nuutinen and J. Ylitalo, “A framework for automatic clustering of parametric MIMO channel data including path powers," IEEE Vehicular Technology Conference, Montreal, Canada, Sep. 2006.

[41] C. Chong, D. Laurenson, and S. McLaughlin, "Statistical characterization of the 5.2 $\mathrm{GHz}$ wideband directional indoor propagation channels with clustering and correlation properties," IEEE 56th Vehicular Technology Conference, vol. 1, pp. 629-633, Fall. 2002.

[42] D. Shutin, "Clustering wireless channel impulse responses in angular-delay domain,"IEEE 5th Workshop on Signal Processing Advances in Wireless Communications, pp. 253-257, Jul. 2004.

[43] M. Kwakkernaat and M.Herben, "Analysis of Clustered Multipath Estimates in Physically Nonstationary Radio Channels", IEEE International Symposium on Personal, Indoor and Mobile Radio Communications, Athens, Greece, Sept. 2007. 
[44] G. Zhang, "Estimation and characterization of delay and angular dispersion on mobile radio channels in $2.25 \mathrm{GHz}$ microcells," Master's Thesis Dissertation, Department of Systems and Computer Engineering, Carleton University, Ottawa, Canada, Apr., 2008.

[45] R. Bultitude, "Measured characteristics of $800 / 900 \mathrm{MHz}$ fading radio channels with high angle propagation through relatively dense foliage," IEEE Journal on Selected Areas in Communications, vol. 5, no. 2, pp. 116-127, Feb. 1987.

[46] E. Sousa, V. Jovanovic, and C. Daigneault, "Delay spread measurements for the digital cellular channel in Toronto," IEEE Transactions on Vehicular Technology, vol. 43, no. 4, pp. 837-847, Nov. 1994.

[47] J. Fessler and A. Hero, "Space-alternating generalized expectation-maximization algorithm," IEEE Transactions on Signal Processing, vol. 42, no. 10, pp. 26642667, Oct. 1994.

[48] B. Fleury, Y. Xuefeng, K. Rohbrandt, P. Jourdan, and A. Stucki, "Performance of a high-resolution scheme for joint estimation of delay and bidirection dispersion in the radio channel," IEEE 55th Vehicular Technology Conference, vol. 1, pp. 522-526, Spring 2000.

[49] B. Fleury, M. Tschudin, R. Heddergott, D. Dahlhaus, and K. Pedersen, "Channel parameter estimation in mobile radio environments using the SAGE algorithm," IEEE Journal on Selected Areas in Communications, vol. 17, no. 3, pp. 434-450, Mar. 1999.

[50] P. Suvikunnas, J. Villanen, K. Sulonen, C. Icheln, J. Ollikainen, and P. Vainikainen, "Evaluation of the performance of multiantenna terminals using a new approach," IEEE Transactions on Instrumentation and Measurement, vol. 55, no. 5 , pp. Oct. 2006.

[51] J. Eklundh, and A. Rosenfeld, "Peak detection using difference operators," IEEE Transactions on Pattern Analysis and Machine Intelligence, vol. PAMI-1, no.3, pp.317-325, July 1979 
[52] M. Molina-Garcia, A. Fernandez-Duran, and J. Alonso, "Application of extreme value distribution to model propagation fading in indoor mobile radio environments," IEEE Radio and Wireless Symposium, pp.97-100, 22-24 Jan. 2008.

[53] J. Cheng, C. Tellambura, and N. Beaulieu, "Performance analysis of digital modulations on Weibull fading channels," IEEE 58th Vehicular Technology Conference, vol. 1, pp. 236-240, 6-9 Oct. 2003.

[54] Sagias, N.C.; Karagiannidis, G.K., "Gaussian class multivariate Weibull distributions: theory and applications in fading channels," IEEE Transactions on Information Theory, vol. 51, no. 10, pp. 3608-3619, Oct. 2005.

[55] T. Sarkar, J. Zhong, K. Kyungjung, A. Medouri, and M. Salazar-Palma, "A survey of various propagation models for mobile communication," IEEE Antennas and Propagation Magazine, vol. 45, no. 3, pp. 51-82, June 2003.

[56] H. Hashemi, M. McGuire, T. Vlasschaert, and D. Tholl, "Measurements and modeling of temporal variations of the indoor radio propagation channel," IEEE Transactions on Vehicular Technology, vol. 43, no. 3, pp. 733-737, Aug. 1994

[57] A. Faugeres, "Multivariate Extremes," in Extreme Values in Finance, Telecommunications, and the Environment, B. Finkenstadt, and H. Rootzen, Ed. USA: CRC press LLC, 2004, pp. 373-388.

[58] C. Chen, and M. Jensen, "A Stochastic model of the time-variant MIMO channel based on experimental observations," IEEE Transactions on Vehicular Technology, vol. 58, no. 6, pp. 2618-2625, July 2009.

[59] I. Telatar, "Capacity of multi-antenna Gaussian channels," European Transactions on Telecommunications, vol. 10, no. 6, pp. 585-595, 1999.

[60] J. Zheng and B. Rao, "Analysis of multiple antenna systems with finite-rate channel information feedback over spatially correlated fading channels, “ IEEE Transactions on Speech and Signal Processing, vol. 55, no. 9, pp. 4612-4626, Sep. 2007. 
[61] J. Roh and B. Rao, "Channel feedback quantization methods for MISO and MIMO systems:' IEEE International Symposium on Personal, Indoor and Mobile Radio Communications, Barcelona, Spin, Sep. 2004.

[62] D. Love, R. Heath, W. Santipach, and M. Honig, "What is the value of limited feedback for MIMO channels?" IEEE Communications Magazine, vol. 42, no. 10, pp. 54-59, Oct. 2004.

[63] J. Roh and E. Rao "An efficient feedback method for MIMO systems with slowly time-varying channels," IEEE Wireless Communications and Networking Conference, Atlanta Georgia, USA, Mar. 2004.

[64] V. Lau, Y. Liu, and T. Chen, "On the design of MIMO block-fading channels with feedback-link capacity constraints," IEEE Transactions on Communications, vol. 52, pp. 62-70, Jan. 2004.

[65] S. Sanayei and A. Nosratinia, "Opportunistic beamforming with limited feedback," IEEE Transactions on Wireless Communications, vol. 6, no. 8, pp. 2765-2771, Aug. 2007.

[66] P. Viswanath, D. Tse, and R. Laroia, "Opportunistic beamforming using dumb antennas," IEEE Transactions on Information Theory, vol. 48, pp. 1277-1294, June 2002.

[67] J. Chung, C. Hwang, K. Kim, and Y. Kim, "A random beamforming technique in MIMO systems exploiting multiuser diversity," Journal on Selected Areas in Communications, vol. 21, pp. 848-855, June 2003.

[68] I. Kim, S. Hong, S. Ghassemzadeh, and V. Tarokh, "Opportunistic beamforming based on multiple weighting vectors," IEEE Transactions on Wireless Communications, vol. 4, no. 11, pp. 2683-2687, Nov. 2005.

[69] M. Sharif and B. Hassibi, "On the capacity of MIMO broadcast channels with partial side information," IEEE Transactions on Information Theory, vol. 51, no. 2, pp. 506-522, Feb. 2005. 
[70] W. Choi, A. Forenza, J. Andrews, and R. Heath, "Opportunistic space division multiple access with beam selection," IEEE Transactions on Communications, vol. 55, no. 12, pp. 2371-2380, Dec. 2007.

[71] N. Zorba, and A. P'erez-Neira, “Opportunistic Grassmannian beamforming for multiuser and multiantenna downlink communications," IEEE Transactions on Wireless Communications, vol. 7, no. 4, pp. 1174-1178, Apr. 2008.

[72] N. Blaunstein, M. Toeltsch, J. Laurila, E. Bonek, D. Katz, P. Vainikainen, N. Tsouri, K. Kalliola, and H. Laitinen, "Signal power distribution in the azimuth, elevation and time delay domains in urban environments for various elevations of base station antenna," IEEE Transactions on Antennas and Propagation, vol. 54, no. 10, pp. 2902-2916, Oct. 2006.

[73] P. Ioannides, and C. Balanis, "Uniform circular arrays for smart antennas," IEEE Antennas and Propagation Magazine, vol. 47, no. 4, pp. 192-206, Aug. 2005.

[74] H. Minn, and V. Bhargava, "An investigation into time-domain approach for OFDM channel estimation," IEEE Transactions on Broadcasting, vol. 46, no. 4, pp. $240-248$, Dec. 2000

[75] Federal Communications Commission, "Spectrum Policy Task Force," Rep. ET Docket no. 02-135, Nov. 2002.

[76] I. Akyildiz, W. Lee, M. Vuran, and S. Mohanty, "NeXt generation/ dynamic spectrum access/cognitive radio wireless networks: a survey," Computer Networks, vol. 50, no. 13, pp. 2127-2159, Sep. 2006.

[77] F. Jondral, "Cognitive radio: A communications engineering view," IEEE Wireless Communication Magazine, vol. 14, no. 4, pp. 28-33, Aug. 2007.

[78] L. Hu, V. Iversen, and L. Dittmann, "Survey of PHY and LINK layer functions of cognitive radio networks for opportunistic spectrum sharing", in Communications and Networking in China, vol. 26, P. Bond Ed., Springer Berlin Heidelberg, 2009, pp. 10-24. 
[79] L. Zhang, Y. Liang, and Y. Xin, "Joint beamforming and power allocation for multiple access channels in cognitive radio networks," IEEE Journal on Selected Areas in Communications, vol. 26, no. 1, pp. 38-51, Jan. 2008.

[80] W. Zhi, Y. Liang and M. Chia, "Robust transmit beamforming in cognitive radio networks", IEEE Conference on Communication Systems, Guangzhou, China, pp. 232-236, Nov. 2008.

[81] X. Lian, N. Nikookar, and J. Zhou, "Adaptive robust beamformers for cognitive radio," The European Wireless Technology Conference, Amsterdam, the Netherlands, pp. 103-106, Oct. 27-31, 2008.

[82] C. Shen, and M. Fitz, "Dynamic spatial spectrum access with opportunistic orthogonalization," the 43rd Conference on Information Sciences and Systems, pp. 600-605,.Baltimore, MD, 18-20 Mar. 2009.

[83] R. Zhang and Y. Liang, "Exploiting multi-antennas for opportunistic spectrum sharing in cognitive radio networks," IEEE Journal of Selected Topics in Signal Processing, vol. 2, pp. 88-102, Feb. 2008.

[84] K. Hamdi, Z. Wei, and K. Letaief, "Joint beamforming and scheduling in cognitive radio networks," IEEE Global Communications Conference, pp. 2977-2981, Washington, DC, Nov. 2007.

[85] Y. Kwon, H. Kim, J. Yoo, and J. Chung, "Orthogonal beamforming methodology in cognitive radio networks," International Conference on Cognitive Radio Oriented Wireless Networks and Communications, Singapore, May 2008.

[86] L. Zhang, Y. Liang, Y. Xin, and H. Poor, "Robust cognitive beamforming with partial channel state information," IEEE Transactions on Wireless Communications, vol. 8, pp. 4143-4153, Aug. 2009

[87] E. Gharavol, Y. Liang, and K. Mouthaan "Robust Downlink Beamforming in Multiuser MISO Cognitive Radio Networks," arXiv:0907.5464v1 [math.OC] 31 Jul 2009 
[88] B. Paul, and R. Bhattacharjee, "Effect of array geometry on the capacity of outdoor MIMO communication: a study," IEEE India Conference, Guwahati , India, Sep. 2006.

[89] C. Gómez-Calero, and R. Martínez-Rodríguez-Osorio, "Influence of array configuration on MIMO channel capacity," Antennas and Propagation Society International Symposium, Madrid, Spain, July 2008.

[90] A. Abouda, H. El-Sallabi, and S. Haggman, "Effect of antenna array geometry and ULA azimuthal orientation on MIMO channel properties in urban city street grid," Progress in Electromagnetics Research, PIER 64, pp. 257-278, 2006.

[91] K. Nishimori, Y. Makise, M. Ida, R. Kudo, and K. Tsunekawa, "Channel capacity measurement of $8 \times 2$ MIMO transmission by antenna configurations in an actual cellular environment," IEEE Transactions on Antennas and Propagation, vol. 54, no. 11 , Nov. 2006.

[92] C. Dietrich, K. Dietze, J. Nealy, and W. Stutzman, "Spatial, polarization, and pattern diversity for wireless handheld terminals," IEEE Transactions on Antennas and Propagations, vol. 49, no. 9, pp. 1271-1281, Sep. 2001.

[93] P. S. Kildal and K. Rosengren, "Electromagnetic analysis of effective and apparent diversity gain of two parallel dipoles," IEEE Antennas Wireless Propagation. Letters, vol. 2, no. 1, pp. 9-13, Dec. 2003.

[94] P. Suvikunnas, J. Salo, L. Vuokko, J. Kivinen, K. Sulonen, and P. Vainikainen, "Comparison of MIMO antenna configurations: methods and experimental results," IEEE Transactions on Vehicular Technology, vol. 57, no. 2, pp. 10211031, Mar. 2008 